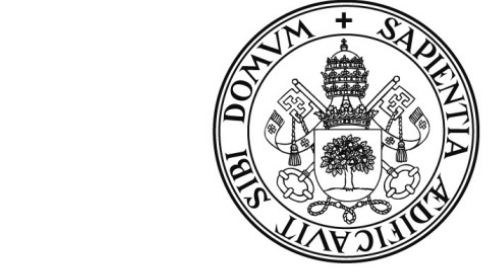

\section{Universidad deValladolid}

FACULTAD DE MEDICINA

INSTITUTO UNIVERSITARIO DE OFTALMOBIOLOGÍA APLICADA

\title{
THE ANTI-INFLAMMATORY COMPOUNDS RESVERATROL AND QUERCETIN AS THERAPEUTIC AGENTS FOR IMMUNE-BASED OCULAR SURFACE DISEASES
}

Compuestos antiinflamatorios Resveratrol y Quercitina como agentes terapéuticos para las enfermedades de base inmune de la superficie ocular

Presentada por Antonio Abengózar Vela para optar al grado de Doctor por la Universidad de Valladolid

Dirigida por:

Dra. María Jesús González García Dra. Amalia Enríquez de Salamanca y Aladro 



\section{AUTORIZACIÓN DEL DIRECTOR DE TESIS}

(Art. 2.1.c de la Normativa para la presentación y defensa de la Tesis Doctoral en la UVa)

La Dra. María Jesús González García, profesor del departamento de Física Teórica, Atómica y Óptica, Centro Facultad de Ciencias, y la Dra. Amalia Enríquez de Salamanca y Aladro, Investigadora Principal del IOBA, Universidad de Valladolid, como Directoras de la Tesis Doctoral titulada "Compuestos antiinflamatorios Resveratrol y Quercitina como agentes terapéuticos para las enfermedades de base inmune de la superficie ocular" "The antiinflammatory compounds resveratrol and quercetin as therapeutic agents for immunebased ocular surface diseases“) presentada por D. Antonio Abengózar Vela, alumno del programa de Doctorado en Ciencias de la Visión impartido por el Instituto Universitario de Oftalmobiología Aplicada (IOBA), de la Universidad de Valladolid autorizan la presentación de la misma, considerando que es APTA para su defensa.

Valladolid, 18 de noviembre de 2014

Las Directoras de la Tesis,

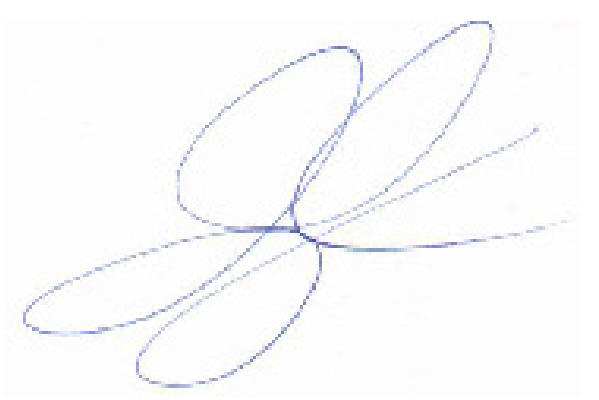

Fdo.: Dra. María Jesús González García

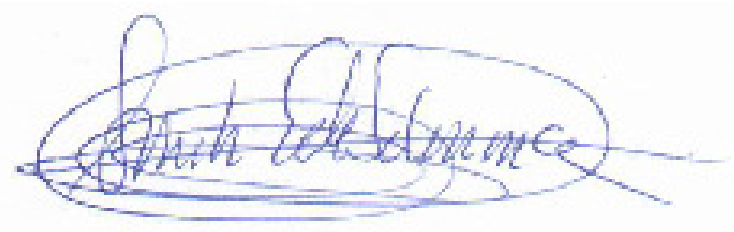

Fdo.: Dra. Amalia Enríquez de Salamanca y Aladro 



\section{CERTIFICATE OF STAY}

UCL Institute of Ophthalmology 11-43 Bath Street London, EC1V 9EL, UK

APPLICANT:

Name: Antonio Abengózar Vela

National Identity Card: $06260086 \mathrm{~S}$

Home Institution: Instituto de Oftalmobiología Aplicada (IOBA), Universidad de Valladolid

\section{HOST INSTITUTION:}

Name: Ocular Biology \& Therapeutics Research Department, UCL Institute of Ophthalmology City and Country: London, United Kingdom

Address: 11-43 Bath Street, London, EC1V 9EL, UK

Responsible person in the host institution: Professor Peter Coffey

Position: Director of the London Project and Professor of Cellular Therapy \& Visual Sciences

This is to certify that the above mentioned person has stayed in this institution in the period from $10 / 09 / 2012$ to $08 / 12 / 2012$.

Within the framework of a collaboration with the University of Valladolid (responsible supervisors María Jesús González-García, PhD and Amalia Enríquez-de-Salamanca, PhD), Mr. Antonio Abengózar-Vela visited my group in the Department of Ocular Biology and Therapeutics in London for a period of three months, working closely with Dr Virginia Calder. During his stay he worked in a joint project between UCL and the University of Valladolid to study the effect of two polyphenols, resveratrol and quercetin, on cord blood mast cells (CBMC) and peripheral blood mononuclear cells (PBMC). He performed proliferation and viability assays on PBMC and cytotoxicity and histamine release assays on CBMC, to study the immune-regulatory and anti-allergic properties of both polyphenols alone and in combination. He was a hard working person, full of enthusiasm and dedicated to the project. He obtained interesting results which will become part of his Thesis and a possible joint paper from both research groups.

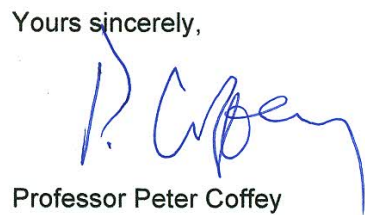

Professor Peter Coffey

Director of the London Projec

Head of Department of Ocular Biology \& Therapeutics,

UCL Institute of Ophthalmology, 11-43 Bath Street, London EC1V 9EL, UK.

Email: p.coffey@ucl.ac.uk

mun thelondonproject. org 

A mi familia 



\section{Agradecimientos/Acknowledgments}

"Cuando realmente deseas algo, todo el universo conspira para que lo consigas". Esta es una de las frases sacadas del libro "El alquimista" de Paulo Coelho que mejor podrían definir la culminación de esta tesis, ya que todo ese universo conspiratorio es la gente sin la cual este proyecto no hubiera podido salir adelante. Así, con estas líneas quiero expresar mi más profundo agradecimiento a todos ellos.

En primer lugar, a mis dos directoras, las doctoras María Jesús González y Amalia Enríquez de Salamanca. Gracias María porque desde el primer día, sin conocerme de nada, te preocupaste de que me sintiera como en casa, tanto fuera como dentro del IOBA (eso es algo que siempre recordaré); y por confiar en mi para realizar esa "idea que te rondaba la cabeza". Gracias Amalia porque siempre me has mostrado tu simpatía y amabilidad; por tu constante ayuda en cada problema experimental y en el trabajo diario de planificación de los experimentos. Agradeceros a las dos vuestra preocupación y cariño en lo personal durante este tiempo. En lo profesional, gracias por vuestros consejos y enseñanzas, apoyo y confianza en todas las etapas de esta tesis; porque habéis contado con mis ideas y humildes aportaciones, algo que un predoctoral siempre agradece de sus directores de tesis y que en pocas ocasiones sucede; y porque siempre habéis tenido la puerta de vuestros despachos abiertas para mi por cualquier motivo, tanto personal como profesional. Gracias por todas las oportunidades que me habéis ofrecido para que yo pudiera asistir a cursos y congresos, para la realización de mis estancias, por la posibilidad de publicar,... Por todo esto y más, igracias!.

Por extensión, quisiera agradecer a "mi tercera directora", la doctora Margarita Calonge, su confianza en la realización de esta tesis y por permitirme formar parte del grupo de superficie ocular (GSO), de lo cual estoy muy orgulloso. Gracias Marga por tu apoyo desde el comienzo de este proyecto, tanto por hacerlo parte de AISWG como por tu implicación en mis estancias que tanto me han aportado personal y profesionalmente.

Gracias también a la doctora Yolanda Diebold por el voto de confianza en aquel estudiante que contactó con ella desde Valencia y al que puso en las manos de María. ¡Seguro que te extrañó ya que no dejabas de repetirme que "el doctorado no es a distancia"!.

Gracias al Profesor José Carlos Pastor porque sin su esfuerzo y dedicación durante tantos años para que el IOBA sea lo que hoy es, un centro de referencia, nada 
de esto podría haberse realizado. El "chico resveratrol" quiere agradecerle el buen humor, el cariño y la confianza que me ha dado durante este tiempo... "y lo sabes".

I also would like to thank Dr. Michael E. Stern and Dr. Virginia Calder for the chance to do my stays in their laboratories, which were two of the best experiences of my life.

Dear Dr. Stern, thank you for your help and recommendations, for showing me how to "look for my lost wallet in a dark street". It has been an honor and privilege for me to have worked in your lab. I really appreciate your involvement in this project, giving me the opportunity to learn from you and your team during my stays in Allergan. Thank you very much for encouraging me to be more confident, not only in my research but also in my life.

Appreciation also goes out to all people who kindly helped me during my stays in Allergan: Chris Schaumburg, Jianping Gao, Kathy Held, Darren Oh, Betty Lee, Sveti Ugarte, Julio Nieves, Annie Ratanapinta, Reuben Sana, Amy Hopkin, Emily Santori, Anh Duong, Kelly Manley and Hsia Edward.

Dear Dr. Calder, thank you very much for your hospitality during my stay in London. I appreciate your advice, supervision and crucial contribution to this thesis... and your patience and efforts to understand my English! I also would like to thank you for opening your home to Gema and me.

I also thank Grazyna Galatowicz for her technical assistance in the lab during my stay at University College London.

I am grateful to Dr. Suzanne Hagan for reviewing the English language in this thesis.

También quisiera dar las gracias a los doctores Roberto Reinoso, Teresa Nieto y Alberto López por su ayuda y apoyo durante estos años. Y a la doctora Itziar Fernández por enseñarme "un poco" de estadística. Gracias a todos por vuestra paciencia infinita a la hora de resolverme dudas. Gracias también a los doctores Alfredo Corell y Carmen Martín por su ayuda y colaboración en los experimentos de citometría de flujo.

Por extensión, gracias a todo el GSO, porque vosotros habéis hecho sentirme en el IOBA como en casa desde el primer día que llegué a Valladolid. En especial, a los pre-doctorales (incluyendo los que ya han "crecido" y son doctores) y técnicos de laboratorio con los que he compartido muy buenos momentos y los que han hecho que cada día fuera especial. Así, quisiera comenzar por alguien que ha estado a mi lado incansablemente en el laboratorio durante todo este tiempo: Carmen García. Carmen, 
gracias por todo el esfuerzo, la generosidad y entrega que has puesto en este trabajo; una parte de esta tesis también te pertenece. Gracias también a ti, Antonio López, porque no solo eres un gran profesional sino una grandísima persona. Al Contact Team, o lo que es lo mismo: José Pinto, Vicente Martín y Alberto López; porque hemos pasado muy buenos ratos juntos tanto en la biblioteca como en los congresos. ¡Qué buenas risas nos hemos echado!. A Sara Galindo, "Sarita", por tu simpatía y los "buenos" momentos que pasamos en Londrés. ¡Vaya experiencia!. Al Nano Team: Laura García, Laura Soriano y Mario Crespo; a Esther Rey, y por supuesto, a los que ya son doctores/doctoras: Laura Contreras, Maria Jesús Benito, Marisa Tesón, Ana de la Mata, Marina López, Isabel Arranz, Amar Kumar y, en especial, a Noelia García; agradeceros todos esos ratos tan fantásticos que hemos vivido. $Y$ como no te iba a agradecer a ti, Mario Martino "Marino", los momentos de confidencias que hemos compartido y las horas que hemos pasado en el gimnasio arreglando el mundo a lo largo de todos estos años.

En general, quisiera dar las gracias a todos los miembros del IOBA con los que en algún momento he podido compartir mi tiempo. Especialmente quisiera agradecer a Ana Rodríguez, Mari Paz García y Lurdes Pérez su profesionalidad y amabilidad durante estos años. Y, por supuesto, no podría olvidarme de ti, Carlos Matesanz. "Charli", gracias por tu profesionalidad, amistad y cariño. Gracias por abrirme las puertas de tu casa y de tu fantástica familia durante estos años.

Tambien quiero dar las gracias a Cristina Raimundo por su ayuda para dibujar alguna de las figuras que en esta tesis se muestran (jeres la mejor!).

Como felizmente ha pasado a lo largo de mi vida, mi familia ha estado a mi lado para apoyarme en todas las decisiones y proyectos que he llevado a cabo; por lo que mi gratitud y recuerdo van dirigidos a todos ellos. En especial, quiero agradecerte a ti, Gema Raimundo, el apoyo que me has ofrecido artruistamente durante estos años. Tu amor, cariño y persistente confianza en mi han sido mi aliento, fuerza y estímulo para seguir adelante y poder culminar este proyecto.

Pero, sobre todo, mi mayor agradecimiento es para mis padres, Antonio y Chelo, los que han sido siempre "mi universo conspiratorio" apoyándome y dándome su cariño y amor incondicional. Gracias papis por vuestro sacrificio y esfuerzo, que han hecho que yo pueda ser lo que soy hoy tanto profesionalmente como personalmente, y de lo cual me siento infinitamente agradecido. Quiero daros mi homenaje particular con estas líneas: si hoy estáis orgullos de mí, más aún lo estoy yo de vosotros. Os quiero. 



\section{Abstract}

The aim of this thesis was to investigate the effect of two major natural compounds, resveratrol and quercetin, and their combination on cells involved in the inflammatory response at the ocular surface, as well as their potential therapeutic effects for dry eye disease.

For this purpose, the anti-inflammatory and anti-oxidant effects of resveratrol, quercetin and their combination were tested in two ocular surface (conjunctival and corneal) epithelial cell lines stimulated by the pro-inflammatory cytokine TNF- $\alpha$ and UV$B$ light. After that, their immunoregulatory effects were determined in activated peripheral blood mononuclear cells. Their anti-inflammatory effects (by topical application) were further studied in a murine model of dry eye induced by desiccating stress, followed by an adoptive transfer model using athymic mice. Additionally, their anti-allergic effects were determined in activated cord blood-derived mast cells.

Results showed that resveratrol, quercetin and their combination can decrease cytokine secretion (IL-6, IL-8/CXCL8 and IP-10/CXCL10) and COX-2 expression induced by TNF- $\alpha$, and free radical production induced by UV-B light in conjunctival and corneal epithelial cells. The combination of both compounds modulated proliferation of peripheral blood mononuclear cells. In addition, both compounds (separately and combined) decreased cytokine production and corneal fluorescein staining in mice exposed to desiccating stress, as well as $\mathrm{CD}^{+} \mathrm{T}$ cell infiltration in conjunctiva of athymic mice. Finally, resveratrol, quercetin and their combination inhibited histamine secretion and cytokine/chemokine production by activated mast cells.

In conclusion, resveratrol, quercetin and their combination can modulate the response of cells involved in inflammatory processes at the ocular surface such as dry eye and ocular allergy. In addition, topical application of resveratrol, quercetin and their combination in experimental dry eye leads to improved clinical signs and inflammation associated with the ocular surface, showing their potential therapeutic properties for treating dry eye disease. 



\section{Financial support}

This research has been made possible thanks to the financial support of Allergan through the agreement: Allergan/IOBA Ocular Surface Inflammation Program, Art. 83 LOU.

I have also received the following support from Universidad de Valladolid:

- Beca de iniciación a la investigación en institutos LOU. 2009.

- Beca para la formación de personal investigador. 2010-2014.

- Ayudas para estancias breves en el desarrollo de tesis doctorales. 2011.

- Ayudas para estancias breves en el desarrollo de tesis doctorales. 2012.

- Ayudas para estancias breves en el desarrollo de tesis doctorales. 2013. 



\section{Publications related to this thesis}

The following publications were a result of data from this doctoral thesis:

\section{Patent:}

Composición para su uso en el tratamiento y/o prevención de la inflamación, el estrés oxidativo y la neovascularización ocular. Número de publicación: P201230664. Asignado a: Universidad de Valladolid and Allergan, Inc. Inventores: María Jesús González García, Amalia Enríquez de Salamanca y Aladro, Margarita Calonge Cano, Michael E. Stern y Antonio Abengózar Vela.

\section{Conference poster presentations:}

Abengózar-Vela A, Schaumburg CS, Stern ME, Calder VL, Calonge M, Enriquez-DeSalamanca A, González-García MJ. An in vivo study of anti-inflammatory effect of Quercetin and Resveratrol polyphenols in a desiccating stress mouse model of dry eye. Annual Meeting of the Association for Research in Vision and Ophthalmology (ARVO). Orlando, Florida, USA. Invest Ophthalmol Vis Sci 2014;55: E-Abstract 3654.

Enriquez-De-Salamanca A, Abengózar-Vela A, García-Vázquez C, Schaumburg CS, Stern ME, Calder VL, Calonge M, González-García MJ. An in vitro study of antiinflammatory effect of Quercetin and Resveratrol polyphenols in ocular surface epithelial cells. Annual Meeting of the Association for Research in Vision and Ophthalmology (ARVO). Orlando, Florida, USA. Invest Ophthalmol Vis Sci 2014;55: EAbstract 1478. 

"Let food be thy medicine and medicine be thy food" Hippocrates of Cos 

Table of content 



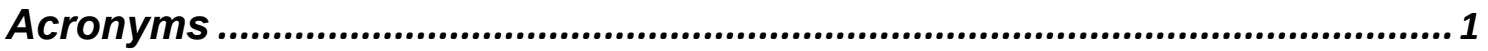

I. Motivation and organisation.......................................................... 5

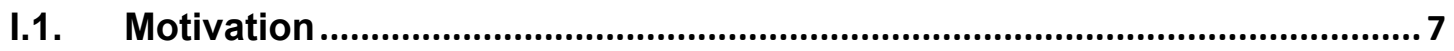

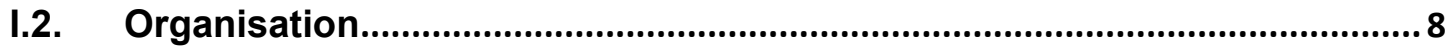

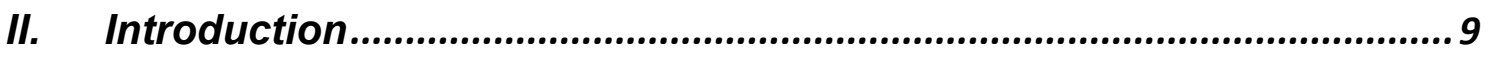

II.1. Definition of the Ocular Surface and Lacrimal Functional Unit ................. 11

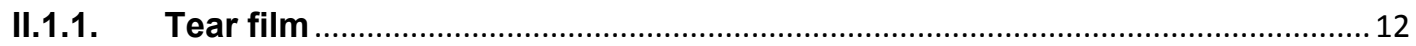

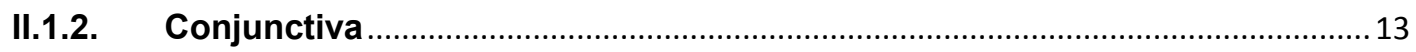

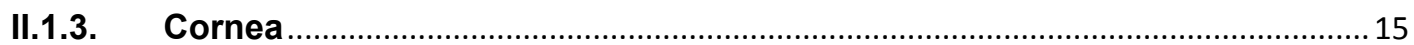

II.2. Ocular Surface Inflammation...................................................................... 16

II.2.1. The Immune System at the Normal Ocular Surface .......................................16

II.2.1.1. Conjunctiva-associated lymphoid tissue ......................................................16

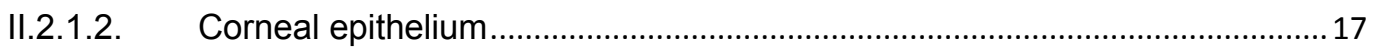

II.2.2. The Inflammatory Process at the Ocular Surface …….................................18

II.3. Acute and Chronic Inflammatory Ocular Surface Diseases ...................... 20

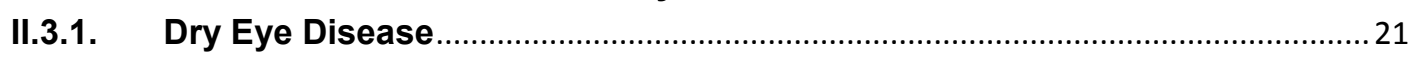



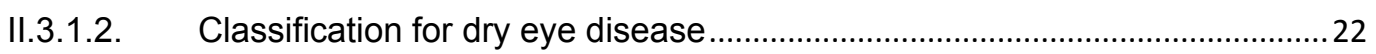

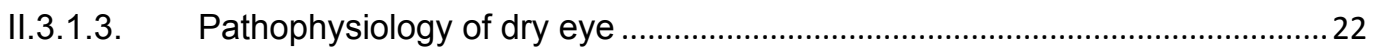

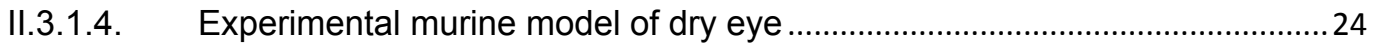

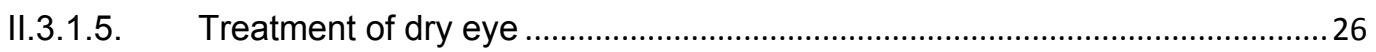

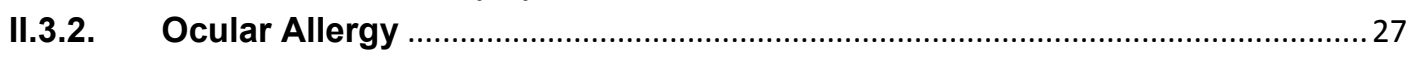

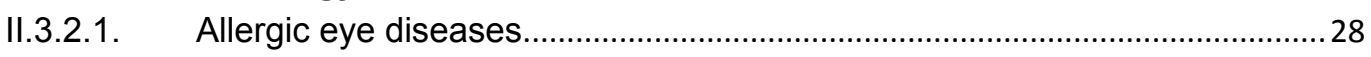

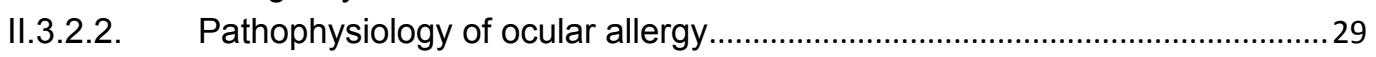

II.3.2.2.1. IgE mediated-hypersensitivity in ocular allergy.........................................29

II.3.2.2.2. T lymphocytes mediated-hypersensitivity in ocular allergy ......................... 31



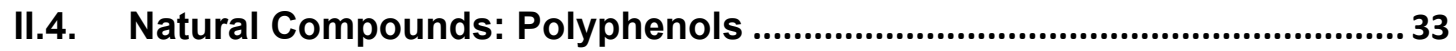

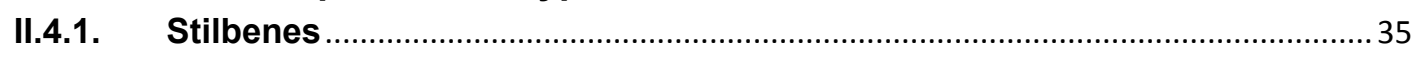

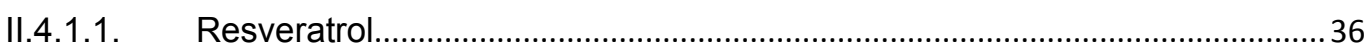

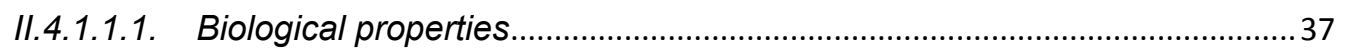

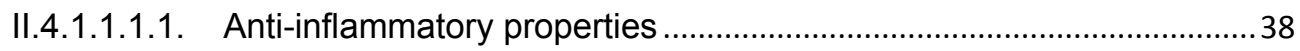

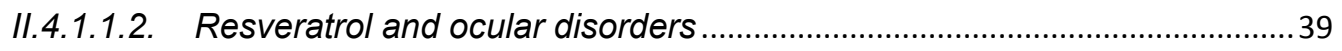

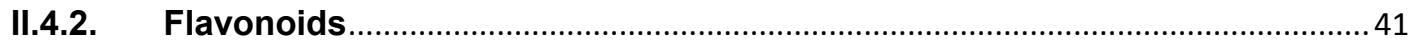

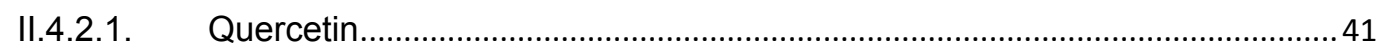



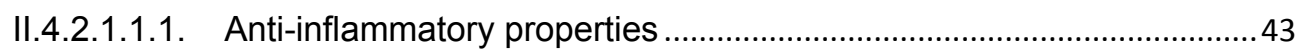

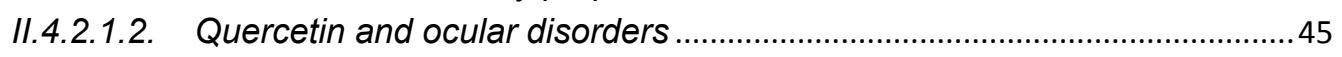

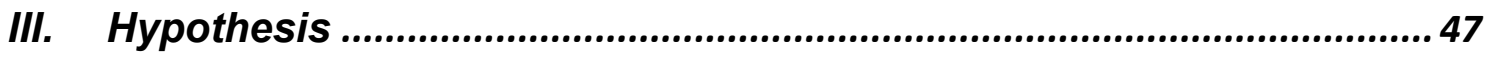

IV. Objectives.................................................................................... 51

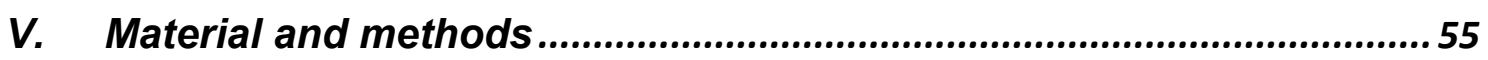


V.1. In-vitro model of inflammation and oxidative stress in human conjunctival and corneal epithelial cells

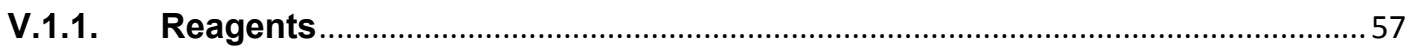

V.1.2. Preparation of polyphenol solutions

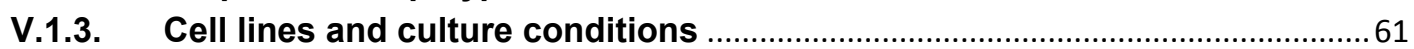

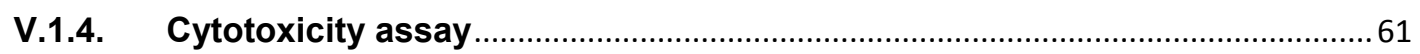

V.1.5. Cell cytokine stimulation and polyphenol treatments ....................................62





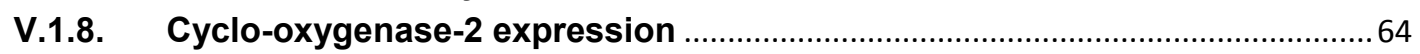

V.1.9. Measurement of reactive oxygen species induced by UV-B radiation...........65

V.2. In-vitro proliferative response of human T cells ........................................66

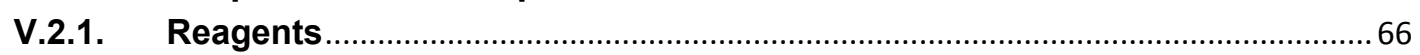

V.2.2. Preparation of polyphenol solutions

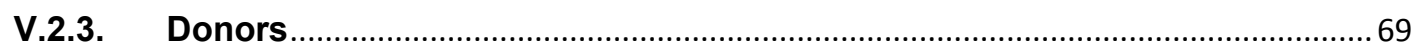

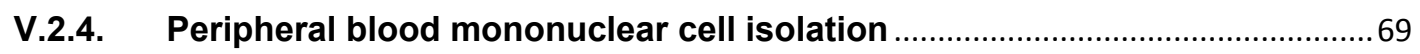

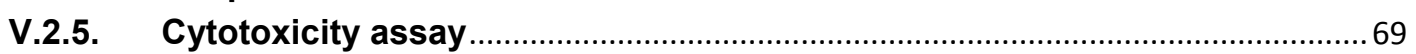



V.3. Experimental murine model of dry eye .......................................................... 71

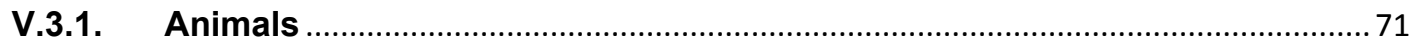

V.3.2. Desiccating stress-induced dry eye and topical treatments ..........................71

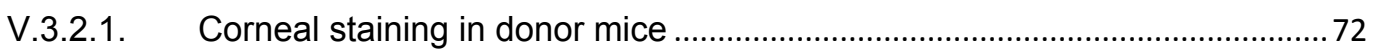

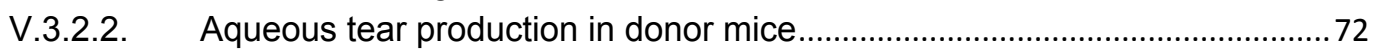

V.3.2.3. Tear collection in donor mice ....................................................................... 73

V.3.2.4. Measurement of cytokine and chemokine levels in donor mouse tears........73

V.3.2.5. Goblet cell count in donor mice ................................................................. 73

V.3.2.6. Immunohistochemistry in donor mice ............................................................. 74

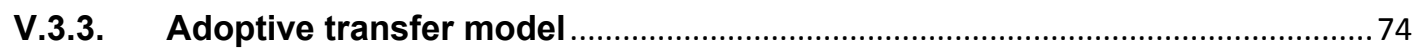

V.3.3.1. Aqueous tear production in recipient mice ...................................................... 75

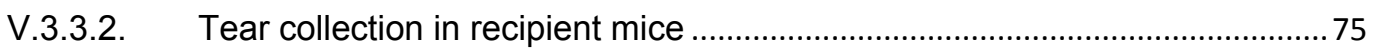

V.3.3.3. Measurement of cytokine and chemokine levels in recipient mouse tears...75

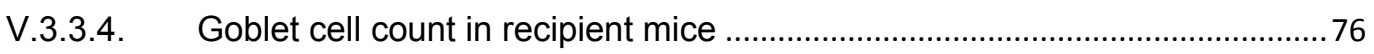

V.3.3.5. Immunohistochemistry in recipient mice ....................................................... 76

V.4. In-vitro model of conjunctival mast cell response..................................... 77

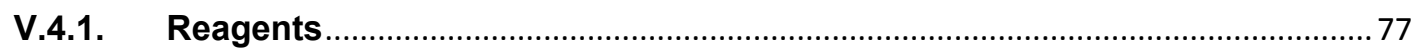

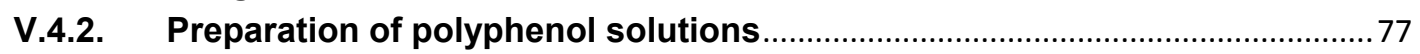

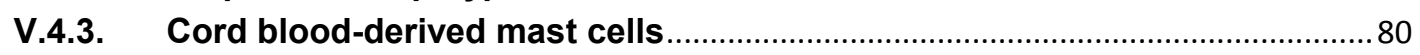

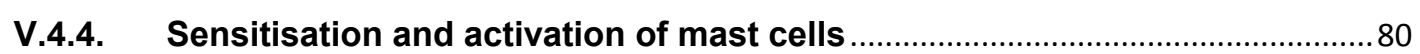

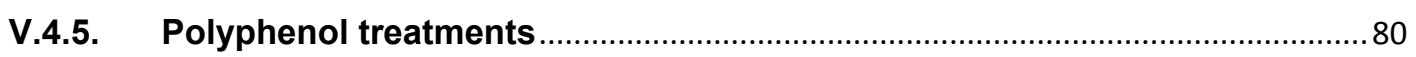

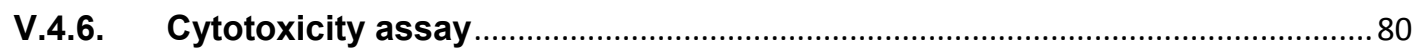

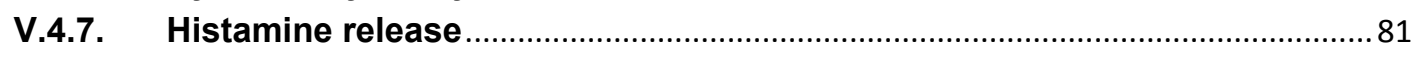

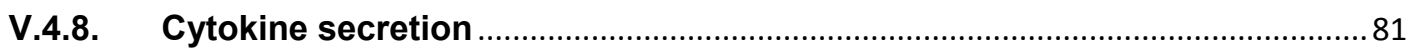

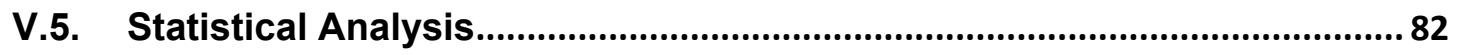

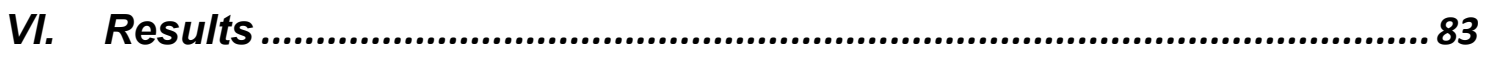

VI.1. In-vitro model of inflammation and oxidative stress in human conjunctival and corneal epithelial cells 
VI.1.1. Cytotoxicity of polyphenols

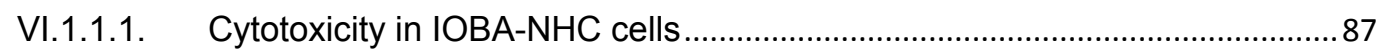

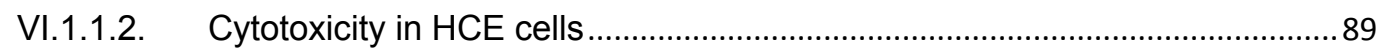

VI.1.2. Effect of Quercetin and Resveratrol on cytokine/chemokine secretion

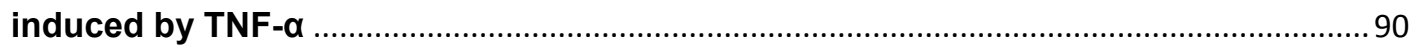

VI.1.2.1. Cytokine/chemokine secretion by IOBA-NHC cells ........................................90

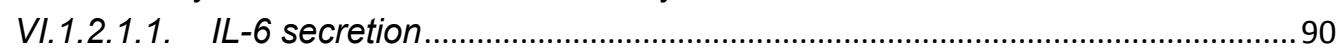

VI.1.2.1.2. IL-8/CXCL8 secretion ............................................................................. 91

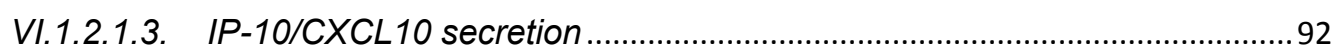

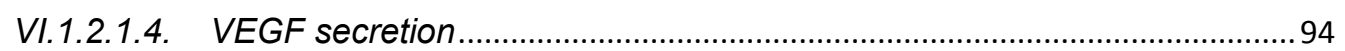

VI.1.2.2. Cytokine/chemokine secretion by HCE cells ..................................................95

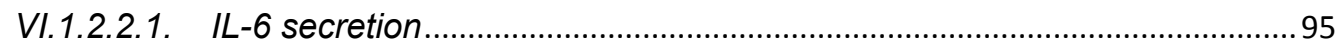

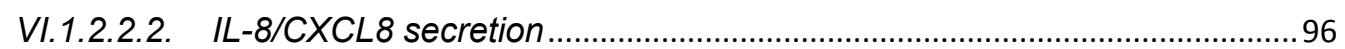

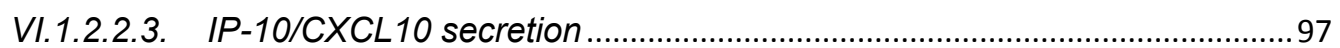

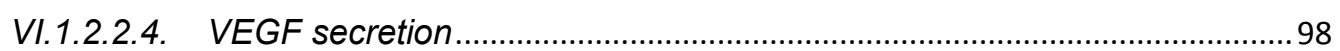

VI.1.3. Effect of Quercetin and Resveratrol on COX-2 expression .............................99

VI.1.3.1. COX-2 expression by IOBA-NHC cells..........................................................99

VI.1.3.2. COX-2 expression by HCE cells...............................................................100

VI.1.4. Reactive oxygen species induced by ultraviolet $B$ radiation.........................102

VI.1.4.1. Intracellular ROS production by IOBA-NHC cells .......................................102

VI.1.4.2. Intracellular ROS production by HCE cells .....................................................103

VI.2. In-vitro proliferative response of human T cells ................................ 105

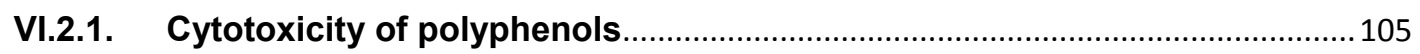

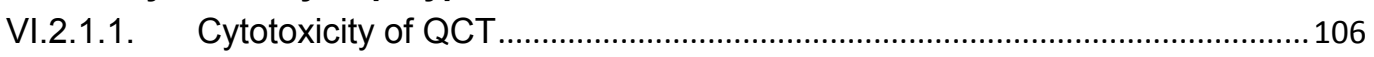

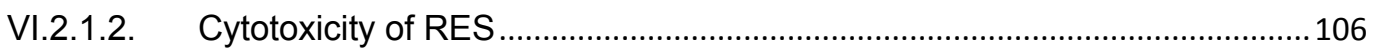

VI.2.1.3. Cytotoxicity of combination of QCT and RES............................................107

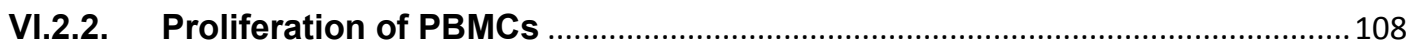

VI.3. Experimental murine model of dry eye and adoptive transfer model 110

VI.3.1. Desiccating stress-induced model of dry eye ….......................................110

VI.3.1.1. Corneal fluorescein staining in donor mice .................................................110

VI.3.1.2. Aqueous tear production in donor mice......................................................111

VI.3.1.3. Cytokine/chemokine concentration in tears of donor mice ..........................112

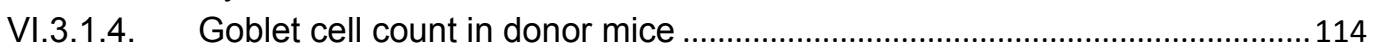

VI.3.1.5. Immunohistochemistry in donor mice ...........................................................115

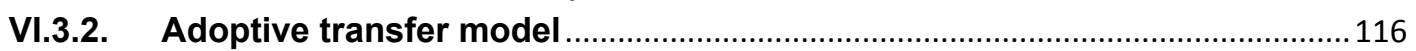

VI.3.2.1. Aqueous tear production of recipient mice....................................................116

VI.3.2.2. Cytokine/chemokine concentration in tears of recipient mice ......................116

VI.3.2.3. Goblet cell count in recipient mice .............................................................. 118

VI.3.2.4. Immunohistochemistry in recipient mice .....................................................118

VI.4. In-vitro model of conjunctival mast cell response .................................. 120



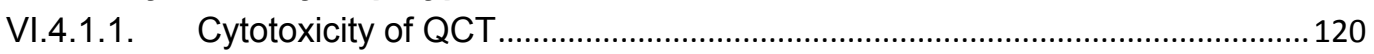



VI.4.1.3. Cytotoxicity of combinations of QCT and RES ...........................................121

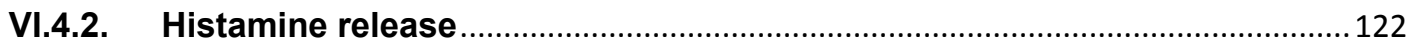

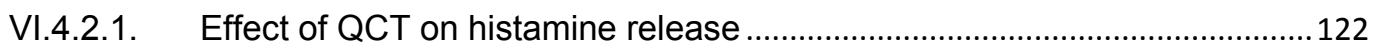

VI.4.2.2. Effect of RES on histamine release ............................................................122

VI.4.2.3. Effect of QCT+RES combination on histamine release ...............................123 


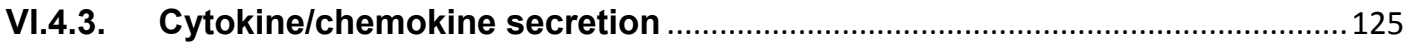

VI.4.3.1. IL-1 $\beta$ secretion ...................................................................................... 126

VI.4.3.2. IL-4 secretion .......................................................................................... 126

VI.4.3.3. IL-5 secretion ......................................................................................... 128

VI.4.3.4. IL-8/CXCL8 secretion ............................................................................ 130

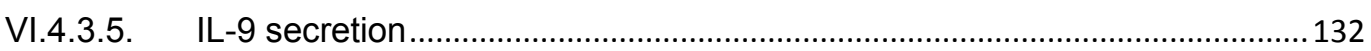

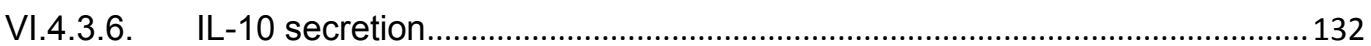

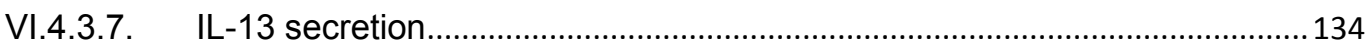

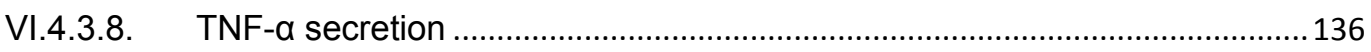



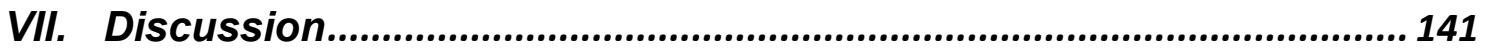

VII.1. In-vitro model of inflammation and oxidative stress in human conjunctival and corneal epithelial cells ............................................................... 145

VII.2. In-vitro proliferative response of human T cells ................................ 155

VII.3. Experimental murine model of dry eye and adoptive transfer mode.159

VII.4. In-vitro model of conjunctival mast cell response .................................. 165

VIII. Summary of thesis results............................................................. 171

IX. Limitations and future studies ......................................................... 177

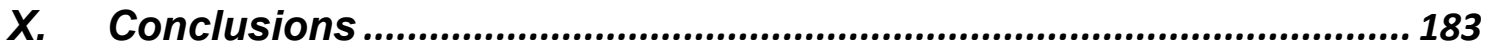

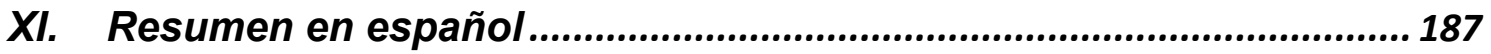

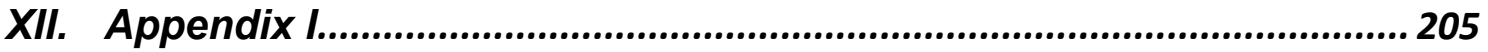

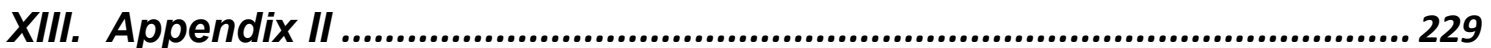

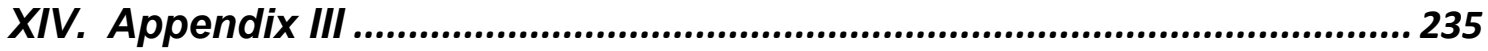

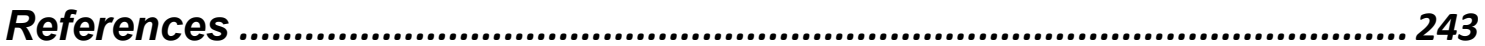




\section{Acronyms}

ADDE

AKC

ANOVA

AP-1

APC

BCA

BSA

CAM

CALT

CBMC

CFS

CFSE

CLN

cox

CM

DC

DED

DEWS

DMEM

DMSO

DS

EALT

EDE

EDTA

EFA

EGF

ELISA

ERK

EtOH
Aqueous tear-deficient dry eye

Atopic keratoconjunctivitis

Analysis of variance

Activator protein 1

Antigen presenting cell

Bicinchoninic acid

Bovine serum albumin

Cell adhesion molecule

Conjunctival-associated lymphoid tissue

Cord blood-derived mast cell

Corneal fluorescein staining

Carboxyfluorescein diacetate succinimidyl ester

Cervical lymph node

Cyclo-oxygenase

Complete medium

Dendritic cell

Dry eye disease

International Dry Eye WorkShop

Dulbecco's Modified Eagle's medium

Dimethyl sulfoxide

Desiccating stress

Eye-associated lymphoid tissue

Evaporative dry eye

Ethylenediaminetetraacetic acid

Essential fatty acid

Epithelial growth factor

Enzyme-linked immunosorbent assay

Extracellular signal-regulated kinase

Ethanol 
FBS

FCS

FDA

FS

GAPDH

GM-CSF

GPC

GSH

HCE

$\mathrm{H}_{2}$ DCF-DA

HLA

$\mathrm{H}_{2} \mathrm{O}_{2}$

HEPES

ICAM

IEL

IFN-Y

$\lg$

IL

iNOS

IOBA-NHC

IP-10/CXCL10

JNK

KIK13

LDALT

LFU

LGALT

LPS

MAPK

MCP-1/CCL2

MCTC

MDA
Foetal bovine serum

Foetal calf serum

Food and Drug Administration

Forward scatter

Glyceraldehyde-3-phosphate dehydrogenase

Granulocyte-macrophage colony-stimulating factor

Giant papillary conjunctivitis

Reduced glutathione

Human corneal epithelium

2',7'-dichlorodihydrofluorescein diacetate

Human leukocyte antigen

Hydrogen peroxide

4-(2-hydroxyethyl)-1-piperazineethanesulfonic acid

Intercellular adhesion molecule

Intraepithelial lymphocytes

Interferon gamma

Immunoglobulin

Interleukin

Inducible nitric oxide synthase

IOBA-normal human conjunctival epithelium

Interferon gamma-induced protein 10

c-Jun N-terminal kinase

Kallikrein 13

Lacrimal drainage-associated lymphoid tissue

Lacrimal functional unit

Lacrimal gland-associated lymphoid tissue

Lipopolysaccharide

Mitogen-activated protein kinase

Monocyte chemoattractant protein-1

Tryptase- and chymase-positive mast cell

Malondialdehyde 
ME

MHC

MMP

MTC

$\mathrm{NaCl}$

$\mathrm{NaHCO}_{3}$

NF-KB

NK

NO

$\mathrm{O}_{2}$

OCT

PAC

PAS

PAGE

PBMC

PBS

PI

PMN

PMSF

PHA

QCT

RES

RFU

ROS

RNS

SAC

SEM

SDS

SOD

SS

TBS
Mercaptoethanol

Major histocompatibility complex

Matrix metalloproteinase

Tryptase-positive mast cell

Sodium chloride

Sodium bicarbonate

Nuclear transcription factor-kappa B

Natural killer cell

Nitric oxyde

Molecular oxygen

Optimal cutting temperature compound

Perennial allergic conjunctivitis

Periodic acid-Schiff

Polyacrylamide gel electrophoresis

Peripheral blood mononuclear cell

Phosphate buffered saline

Propidium iodide

Polymorphonuclear leukocytes

Phenylmethanesulfonyl fluoride

Phytohemagglutinin

Quercetin

Resveratrol

Relative fluorescence units

Reactive oxygen species

Reactive nitrogen species

Seasonal allergic conjunctivitis

Standard error of mean

Sodium dodecyl sulphate

Superoxide dismutase

Side scatter

Tris-buffered saline 
TCR

TGF- $\beta$

Th

TLR

TP

Treg

TNF- $\alpha$

UV

VCAM

VKC

WHO

8-OHdG
T cell receptor

Transforming growth factor beta

$\mathrm{T}$ helper cell

Toll-like receptor

Tear production

Regulatory T cell

Tumor necrosis factor alpha

Ultraviolet light

Vascular cell adhesion molecule

Vernal keratoconjunctivitis

World Health Organization

8-hydroxydeoxyguanosine 
I. Motivation and organisation 



\section{I.1. Motivation}

Acute and chronic inflammatory ocular surface diseases affect millions of people around the world and the costs to treat these, for example dry eye, are substantial. In addition, quality of life in patients suffering dry eye has been likened to moderate-to-severe angina, even when clinical signs are minimal. The treatments of dry eye include artificial tears, cyclosporine $A$ and corticosteroids. Artificial tears do not have any anti-inflammatory effect; and topical cyclosporine $A$ and topical methylprednisolone corticosteroid have been associated with improvements in clinical signs and symptoms reported by dry eye patients, but those treatments either have side-effects (corticosteroids) or are only available in some countries (cyclosporine A). Thus, effective management strategies would be welcomed by clinicians and patients, because there is a gap between artificial tears and the anti-inflammatory therapies.

In addition, not only inflammation plays a key role in acute and chronic diseases. There is a close relationship between oxidative stress and inflammation because an increase in free radical production is able to activate the inflammatory process, and free radicals are also effectors of inflammation. As a consequence of oxidative stress, signal transduction cascades can stimulate the production of proinflammatory cytokines. The oxidative stress in the ocular surface has been evidenced in several diseases such as conjunctivochalasis, ${ }^{1}$ ocular allergy ${ }^{2}$ and dry eye, ${ }^{3}$ demonstrating that oxidative stress and inflammation are intimately related.

This close relationship between oxidative stress and inflammation suggests that compounds with both antioxidant and anti-inflammatory properties may be a new approach for the treatment of ocular surface disorders, such as dry eye and ocular allergy.

Natural compounds, such as polyphenols, have been well described in the literature as therapeutic compounds by their unique biological properties. Resveratrol and quercetin, two major polyphenols, are promising natural anti-inflammatory and antioxidant agents because they have beneficial effects in many inflammatory conditions. Moreover, these compounds have various effects on different target cells involved in inflammation.

Thus, the aim of this thesis was to determine the potential effect of two polyphenols, resveratrol and quercetin, and their combination on maintenance of ocular surface health in inflammatory diseases, such as dry eye and ocular allergy. 


\section{I.2. Organisation}

This Doctoral Thesis applies for the International-awarded Doctorate Degree. It has been performed at the University of Valladolid, under the regulations of the International Doctorate Committee. The joint requirements are as follows: the whole manuscript has been written in English and a general summary in Spanish, in which the objectives, methodology and summary of results are presented.

This Thesis report is organised in four sections according to each model used for testing polyphenols as follow: 1) in-vitro model of ocular surface inflammation, 2) invitro model of $\mathrm{T}$ cell proliferation, 3) murine model of dry eye disease and 4) in-vitro model of conjunctival mast cells. This thesis matches the requirements for the International-awarded Doctorate Degree with the sections V.2 and V.4, in which the experiments were performed during my stay in the research group of Dr. Virginia Calder at University College London (UCL). In addition, the patent from this thesis and achieved results from additional experiments carried out during my stay in the research group of Dr. Michael E. Stern at Allergan, Inc. (in which the section V.3 was performed) are included in the Appendices. 


\section{Introduction}





\section{II.1. Definition of the Ocular Surface and Lacrimal Functional Unit}

The ocular surface is an important part of the eye not only by being a vital component of the refractive media of the eye but also by protecting the delicate inner structures of the eye. Unlike all other wet surface epithelia of the body, the ocular surface is exposed to the outside environment where it is especially subjected to pathogens, adverse environments and injury. As a result, several protective mechanisms are involved in the ocular surface to conserve corneal transparency and maintain a good refractive surface.

The ocular surface is an integrated unit comprising the cornea, the conjunctiva, the limbus and the overlying tear film. Moreover, it is also well known that all these components are interconnected through a continuous epithelium with no breaks between regions and neuroanatomically connected with the nervous, vascular, immune and endocrine systems. Based on this idea of interconnected tissues, Stern et al. described the term "Lacrimal Functional Unit" (LFU) in $1988 .{ }^{4}$ They defined the LFU as an integrated system that connects the sensory tissues and the secretory glands to regulate tear secretion maintaining homeostasis of the ocular surface. This definition emphasises and highlights the close relationship between the lacrimal gland, the ocular surface and the nervous system. Thus, the LFU is composed of the ocular surface, the main and accessory lacrimal glands and the neural network that connects them ${ }^{4}$ (Figure 1).

The main role of the LFU is to provide a normal and stable tear film leading to a smooth optical surface, so maintaining comfort, epithelial cell health and protection from environmental and microbial insults. ${ }^{5}$ Nevertheless, dysfunction of, or injury to, some element of the LFU can alter the tear film structure and composition. This event may result in both corneal and conjunctival epithelia being directly exposed to the external environment, so losing the nourishment and lubrication provided by the tear film. As a result, inflammatory processes occur in the LFU, leading to the development of ocular surface disorders, such as dry eye disease (DED).

The treatment of the inflammatory process in the ocular surface mainly relies on topical drugs because of the easy access to the ocular surface epithelium. It focuses on initially treating the inflammatory process associated with the corneal and conjunctival epithelia. Therefore, it is necessary to understand the structure and immune response in both normal and pathological epithelia, in order to find therapeutic targets to treat ocular surface disorders. 


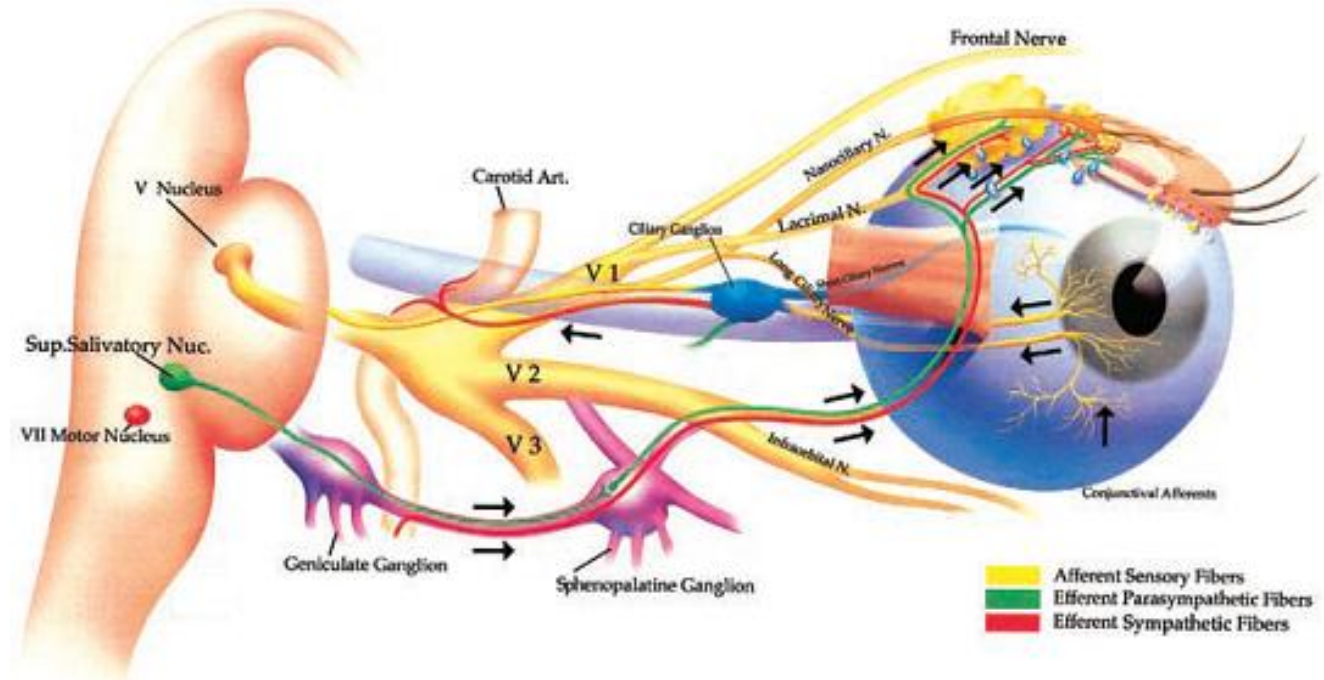

Figure 1. The lacrimal functional unit. Adapted from Pflugfelder et al. $2004{ }^{6}$

\section{II.1.1. Tear film}

As mentioned above, the tear film plays a key role in maintaining the homeostasis of the ocular surface. The traditional structure of the tear film comprises three layers: an outer lipid layer, an intermediate aqueous layer and an inner mucin layer. However, some studies have evidenced that there is no real clear-cut barrier between the layers. ${ }^{7,8}$ Thus, it is currently proposed that a large portion of the tear film is a mucin/aqueous gel that decreases in density towards the external lipid layer ${ }^{9,10}$ (Figure 2). Nevertheless, three components will be described separately here for simplicity.

The lipid layer is secreted by the meibomian glands. This layer is made of a mixture of lipids that prevent evaporation of the aqueous layer and provide some of the refractive qualities necessary for a good optical surface.

The aqueous layer is secreted by the lacrimal gland and accessory glands. This layer is an aqueous-mucin gel containing water, electrolytes and proteins that prevent corneal and conjunctival infections. The major locally-produced proteins are lysozyme, tear albumin, lipocalin, secretory immunoglobulin (Ig) A and lactoferrin, ${ }^{11}$ which are increased during inflammation. ${ }^{12,13}$ In addition, there are also proteins and peptides belonging to the immune system, ${ }^{14}$ such as $\beta$-defensins, ${ }^{15}$ which either have antimicrobial effects or serve to recruit and activate lymphoid cells.

The mucin layer is secreted by goblet cells, along with conjunctival epithelial cells. It is a glycocalyx gel made of a heterogeneous glycoprotein group that promotes tear film attachment to the corneal epithelium. Mucins maintain lubrication and stabilise both lipid and aqueous components of the tear film, thus avoiding desiccating stress at 
the ocular surface. ${ }^{16}$ In addition, the mucin layer of the tear film also provides a physical barrier that restricts bacterial adherence. ${ }^{17}$



Figure 2. The normal tear film structure and components. Adapted from Stern et al. $2004 .^{5}$

\section{II.1.2. Conjunctiva}

The conjunctiva is a thin and translucent mucous membrane which extends from the eyelid margin to the corneal limbus, including the nasal mucosal through the lacrimal puncta. Although the conjunctiva is a continuous membrane, it can be anatomically divided into three parts: palpebral, forniceal and bulbar conjunctiva (Figure 3).

The palpebral conjunctiva (also called the tarsal conjunctiva) starts on the lids at the mucocutaneous junction and ends at the forniceal conjunctiva. It is highly vascularised and firmly adhered to the tarsal plate.

The forniceal conjunctiva, also called the cul de sac, is a transition zone between the tarsal and bulbar conjunctiva. It is the loosest of the three conjunctival areas and is reflected into several folds. The ducts of the main lacrimal gland and Krause and Wolfring accessory glands open into the forniceal conjunctiva. 


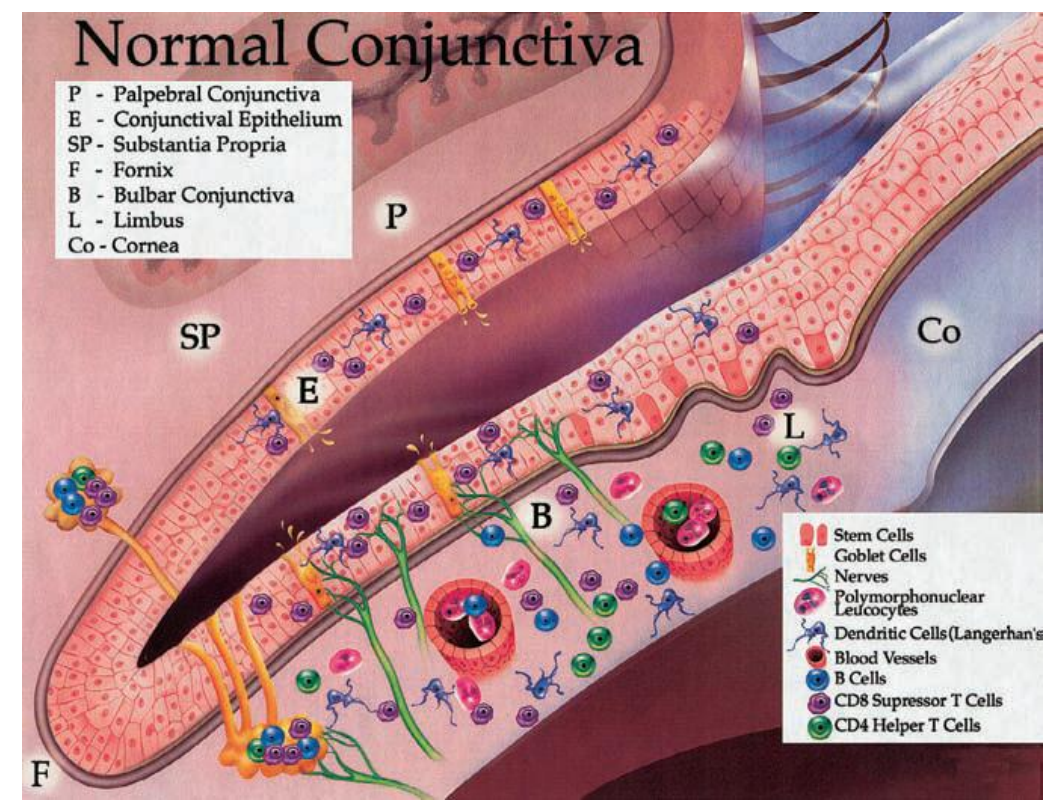

Figure 3. Main components of the normal conjunctiva. Adapted from Calonge et al. 2004. ${ }^{18}$

The bulbar conjunctiva envelops the anterior sclera. It can be further divided into two portions: scleral conjunctiva (from the fornix to the limbus) and limbal conjunctiva (a ring approximately $3 \mathrm{~mm}$ around the cornea in the transition zone between the corneal epithelium and conjunctival epithelium). The bulbar conjunctiva is tightly bound to the underlying Tenon's capsule at the limbus but it is loosely attached to the globe.

Histologically, the conjunctiva consists of an epithelium and an underlying loose connective tissue, further subdivided into the substantia propria and a deeper fibrous layer. The conjunctival epithelium is a non-keratinised, stratified secretory layer and its structure varies depending on region. Epithelial cells are columnar in the tarsus, cuboidal in the bulbar conjunctiva, prismatic in the fornix, and squamous in the limbus and near the lids. Its thickness also varies regionally, from 2-3 layers in the tarsus, to 69 layers in the bulbar conjunctiva. ${ }^{18}$ It contains mucous-secreting goblet cells, and cells involved in the defensive response, such as melanocytes, lymphocytes and antigen presenting cells (APCs) called Langerhans cells, as well as major histocompatibility complex (MHC) class II positive dendritic cells (DCs).

The superficial lymphoid layer is a connective tissue under the epithelium, which is not present at birth (it takes three months to develop), and it is loosely attached to the overlying epithelial base membrane. This tissue contains the immune system cells, such as lymphocytes, macrophages, natural killer (NK) cells, neutrophils and mast cells, which initiate and mediate the inflammatory response in the ocular surface. 
The deeper fibrous layer is a thick layer, except for the tarsal part of the conjunctiva, which contains the majority of conjunctival blood vessels and nerves. The accessory lacrimal glands of Krause and Wolfring are also located within this fibrous layer.

\section{II.1.3. Cornea}

The cornea is the principal refractive component of the eye. The anterior surface of the cornea is bathed by the tear film and the posterior surface lines the aqueous humour-filled anterior chamber. The thickness of the cornea is approximately $500 \mu \mathrm{m},{ }^{19}$ increasing towards the peripheral limbus. Peripherally, the cornea lines the sclera at the region called the corneal sulcus. The cornea has the highest concentration of non-myelinated nerve fibres of the human body. These fibres are associated with numerous pain receptors that have a very low threshold, making the cornea highly sensitive to pain. As the cornea is an avascular tissue, both the tear film and aqueous humour (along with capillaries at the edge of the cornea) provide the necessary oxygen and nutrients.

Anatomically, the cornea is made up of 5 layers, each with distinct functions (Figure 4). The layers are from front to back: epithelium, Bowman's membrane, stroma, Descemet's membrane and endothelium. From all of these structures, the epithelium is the outer-most layer of the cornea. It is a non-keratinised, stratified squamous tissue that presents a physical barrier to prevent microorganisms from reaching the inner corneal layers. The central corneal epithelium is $50-55 \mu \mathrm{m}$ thick with $5-6$ cell layers, reaching 10 cell layers or more towards the limbus. The epithelium has 3 types of cells: two layers of flattened epithelial cells externally (squamous cells), followed by 2-3 layers of polygonal cells (wing cells), and a single internal basal layer of columnar cells (basal cells). The basal cells or stem cells located in the palisades of Vogt are responsible for mitosis. The new cell originates as another basal cell and migrates upwards, eventually becoming a wing cell. Upward movement continues towards the centre of the cornea, until it finally becomes an outer epithelial cell and is eventually shed. The external cell layer also has microvilli and microplicae that play a role in tear film adhesion and stabilisation to the corneal surface. Running between the epithelial cells are the ends of sensory nerve fibres. 


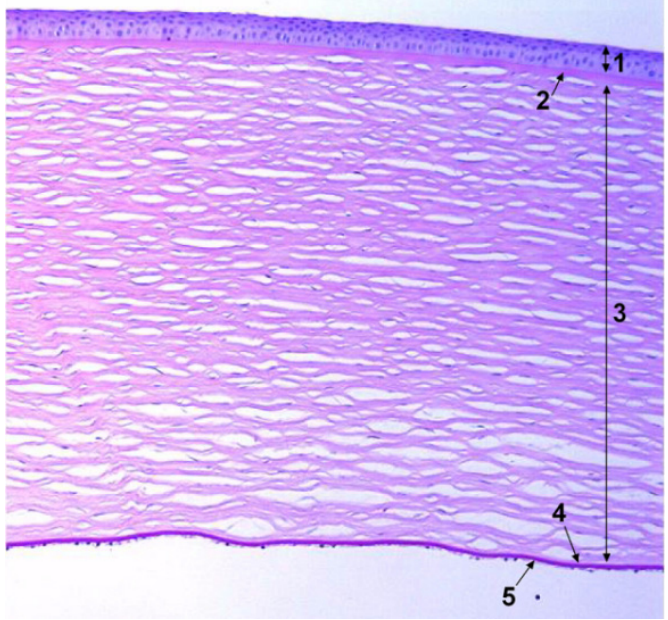

Figure 4. Cross section of the human cornea. Image courtesy of IOBA Ocular Pathology Lab. (1: Epithelium; 2: Bowman's membrane; 3: Stroma; 4: Descemet's membrane; 5: Endothelium).

\section{II.2. Ocular Surface Inflammation}

Most of the acute and chronic ocular surface disorders include a significant component of the inflammatory response. Therefore, it is very important to understand the cellular and molecular mechanisms involved in immune-based ocular surface diseases, in order to identify new therapeutic targets for treating them.

\section{II.2.1. The Immune System at the Normal Ocular Surface}

The ocular surface contains an immunological framework that limits host tissue damage when it is insulted by pathogens or environmental trauma. Conjunctivaassociated lymphoid tissue and the corneal epithelium are the two major components of this immunological framework at the ocular surface.

\section{II.2.1.1. Conjunctiva-associated lymphoid tissue}

As mentioned, there are several types of immune cells in the normal epithelium of the ocular surface (Table 1). The conjunctiva, along with the lacrimal gland and lacrimal drainage system, form a continuous lymphoid tissue called eye-associated lymphoid tissue (EALT) ${ }^{20}$ The EALT consists of the lacrimal gland-associated lymphoid tissue (LGALT), the conjunctiva-associated lymphoid tissue (CALT) and the lacrimal drainage-associated lymphoid tissue (LDALT). The EALT is connected to the other 
immune system tissues by the recirculation of lymphoid cells via specialised vessels. Therefore, lymphoid cells are resident at the normal conjunctival epithelium.

Focusing on the CALT, it has the typical characteristic distribution found in other lymphoid tissues in mucosal organs: diffuse lymphoid tissue and lymphoid follicles. ${ }^{21}$

Diffuse lymphoid tissue contains intraepithelial lymphocytes (IEL) in the basal layer of the epithelium and in lamina propria. IEL are predominantly $\mathrm{CD}^{+} \mathrm{T}^{-}$cells compared to $\mathrm{CD} 19^{+} \mathrm{B}$ cells. $\mathrm{T}$ cells are predominantly $\mathrm{CD} 3^{+} \mathrm{CD} 8^{+}$suppressor/cytotoxic $\mathrm{T}$ cells compared to $\mathrm{CD} 3^{+} \mathrm{CD} 4^{+} \mathrm{T}$ helper (Th) cells, except for in the lamina propria, where a reverse distribution of $\mathrm{T}$ cells occurs. ${ }^{22}$ Lymphocytes are complemented by bone marrow derived cells such as APCs, mast cells and macrophages, including IgAsecreting plasma cells.

Lymphoid follicles produce lymphoid effector cells and can be observed in the normal conjunctiva. They consist of $B$ cells surrounded by $T$ cells and associated high endothelial venules which support high levels of lymphocyte extravasation from the blood.

\begin{tabular}{|c|c|c|c|}
\hline \multirow[b]{2}{*}{ Cell type } & \multicolumn{2}{|c|}{ Conjunctiva } & \multirow{2}{*}{$\begin{array}{c}\text { Cornea } \\
\text { Epithelium }\end{array}$} \\
\hline & Epithelium & Substantia propria & \\
\hline Basophils & - & - & - \\
\hline Dendritic cells & + & + & + \\
\hline Eosinophils & - & - & - \\
\hline \multicolumn{4}{|l|}{ Lymphocytes } \\
\hline$B$ cells & - & + & Rare \\
\hline$T$ cells & + & + & + \\
\hline$\alpha / \beta$ & + & + & $?$ \\
\hline $\mathrm{\gamma} / \delta$ & + & + & $?$ \\
\hline T-helper & + & + & Rare \\
\hline$T$-suppressor & + & + & Rare \\
\hline Macrophages & - & + & - \\
\hline Mast cells & - & + & - \\
\hline Plasma cells & - & + & - \\
\hline Polymorphonuclear & & & \\
\hline Leucocytes & - & + & - \\
\hline
\end{tabular}

\section{II.2.1.2. Corneal epithelium}

Although the corneal epithelium is a continuation of the conjunctival epithelium, it is specialised to exist over an avascular connective tissue. Lymphoid and blood 
vessels end in the limbal zone and prevent an access of the vast majority of immunologically relevant cells into the cornea. This alymphatic and avascular characteristic leads to an "immune privilege" that retards trafficking of immune cells to the lymphoid tissues.

Nevertheless, some immune cells are located in the normal corneal epithelium. APCs are present in the corneal epithelium. There are different populations located in both central and peripheral epithelia; ${ }^{24,25}$ declining in percentage from the limbus to centre of the corneal epithelium. ${ }^{26,27}$ Peripheral APCs are mainly Langerhans cells, whilst central APCs are immature epithelial DCs ${ }^{25}$ (Figure 5). In addition, B and T cells are also present in the limbic region. ${ }^{28}$ To date, no other immune cell types have been described in the normal corneal epithelium.

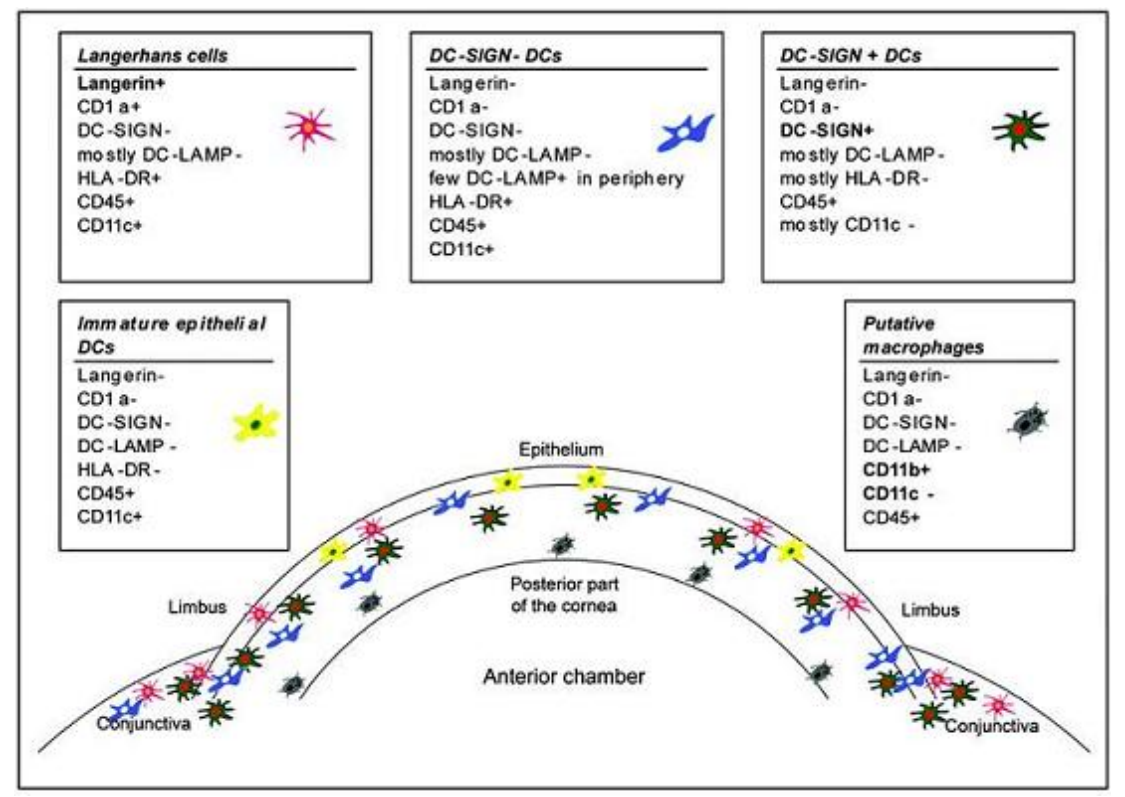

Figure 5. Distribution of antigen presenting cells within the central and peripheral cornea (DC: Dendritic cells). Adapted from Mayer et al. $2007 .^{25}$

\section{II.2.2. The Inflammatory Process at the Ocular Surface}

The ocular surface has the same immune cell response as other mucosal epithelia in the human body. Therefore, both arms of the immune system, innate and adaptive systems, are the main features against infectious agents.

The innate response is the first line of defence against invading microbial pathogens. This immune system response occurs immediately, but in a non-specific manner, to invading pathogens because it recognises general classes of pathogens (i.e. virus, bacteria and fungi), but it cannot make fine distinction. It consists of the 
physical and chemical barriers, macrophages, DCs, NK lymphocytes and circulating plasma proteins. The physical barriers of the ocular surface epithelia (i.e. corneal epithelial cells), along with mucous membranes (i.e. conjunctiva), secreted proteins (i.e. lysozyme and defensins) and mechanical barriers (i.e. eyelids) are just some features of the innate immune system at the ocular surface (Figure 6). The integrity of the ocular surface epithelium is provided by tight junctions between cells. In addition, epithelial cells can recognise pathogen-associated molecular pattern by Toll-like receptor (TLR) expression on their surfaces.

However, if pathogens manage to bypass, evade or overwhelm the innate defences, the adaptive immune response is necessary for effective elimination of pathogens. The adaptive or acquired immune system consists of two arms: humoral (specific antibodies secreted by B lymphocytes) and cellular (specific T lymphocytes).

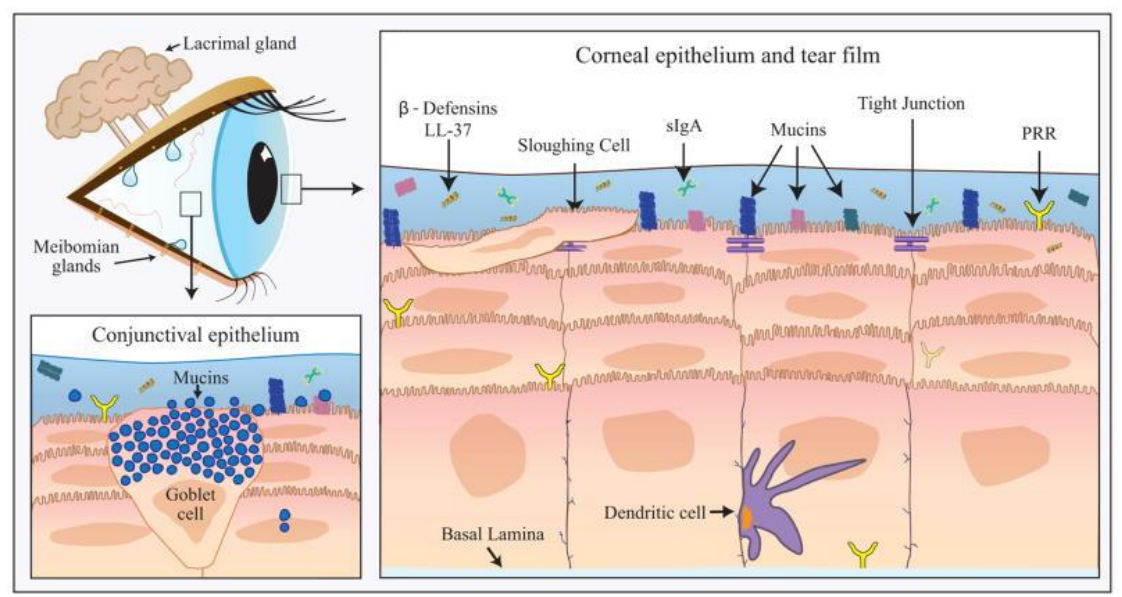

Figure 6. Innate immune system of the ocular surface (PRR: Pattern recognition receptors; LL-37: Cathelicidin; IgA: Immunoglobulin A). Adapted from Narayanan et al. 2013. ${ }^{29}$

Key links between the innate and adaptive immune systems are APCs (i.e. B cells, macrophages and DCs). Activated APCs migrate to draining lymphatic tissue (such as regional lymph nodes and spleen) via afferent lymphatic vessels. They have the function of priming naïve $T$ cells in these lymphoid tissues, leading to differentiation and activation of effector T cells. Once the $T$ cells are activated, the presence/absence of cytokines (signalling molecules that mediate intercellular communication like lymphocytes and epithelial cells), such as interleukin (IL)-12p70, IL-4, IL-6 and transforming growth factor (TGB)- $\beta$, determine the fate of naïve $T$ cells as Th cells (CD4 ${ }^{+} \mathrm{T}$ cells). They can differentiate into Th1, Th2, Th9, Th17 and Th22 cells, and regulatory $\mathrm{T}$ cells (Treg), all $\mathrm{CD} 4^{+} \mathrm{T}$ cell subtypes. The $\mathrm{T}$ cell subsets promote different types of inflammatory responses depending on their effector cytokine profiles and 
interactions with resident tissue cells. $T$ cells can be distinguished from other lymphocytes such as B cells and NK cells by the presence of T cell receptor (TCR) on the cell surface.

Th1 cells secrete IL-2 and interferon (IFN)- $\mathrm{y}$. These cytokines play a role upregulating production of chemokines and cell adhesion molecules (CAMs) that further attract inflammatory cells, for example to the ocular surface, and also promote maturation of macrophages. ${ }^{30}$ Therefore, Th1 lymphocytes are pro-inflammatory cells involved in the pathogenesis of some autoimmune diseases. ${ }^{31}$

Th2 cells produce inflammatory cytokines IL-4, IL-5 and IL-13. These cytokines are involved in the development of atopic diseases, including seasonal allergy, asthma and atopic dermatitis/keratoconjunctivitis. IL-4 and IL-13 promote IgE switching by B cells, ${ }^{30,32}$ and IL-5 participates in the activation and chemotaxis of eosinophils. ${ }^{33}$ Th2 cell activation occurs in allergic conjunctivitis. ${ }^{30}$

Th17 cells are involved in autoimmunity and tissue inflammation. ${ }^{34}$ They particularly secrete $\mathrm{IL}-17,{ }^{31}$ which stimulates production of cytokines (i.e. tumor necrosis factor (TNF)- $\alpha$ and IL-6), chemokines (i.e. IL-8/CXCL8 and interferon gammainduced protein (IP)-10/CXCL10) and matrix metalloproteinases (MMPs) on a broad range of cell types. ${ }^{35}$ In the ocular surface, DED elicits Th1 and Th17 responses. ${ }^{36}$

Treg cells have a role in tolerance, rather than immunity and secrete IL-10 and TGF- $\beta{ }^{37,38}$ In addition, it has been described that Tregs play a key role as immunomodulatory T cells in experimental DED. ${ }^{39-41}$

Th9 cells secrete IL-9 and are closely related to Th2 cells. ${ }^{42}$ Th22 cells produce IL-22. ${ }^{43}$ Both cells may contribute to the development of chronic allergic inflammation and autoimmune disease. ${ }^{44-46}$ The role of Th9 and Th22 in eye diseases is currently under investigation. ${ }^{47}$

\section{II.3. Acute and Chronic Inflammatory Ocular Surface Diseases}

Acute inflammation is a complex and essential early response, protecting tissue integrity and homeostasis after minor injuries like burns and cuts, as well as in major trauma. Acute inflammation is characterised by the rapid appearance of signs such as heat, swelling, pain, redness and loss of function. ${ }^{48}$ One of the most common acute processes at the ocular surface is acute allergic conjunctivitis.

If acute inflammation is not resolved over time it can evolve into a chronic process. Chronic inflammation is characterised by prolonged duration (weeks or months, even years) caused by persistent infections, prolonged exposure to toxic reagents or autoimmune-mediated inflammatory disease. In autoimmune diseases, the 
inflammatory response is triggered when there are no stimuli and the immune system attacks its own tissues. Some eye diseases such as vernal keratoconjunctivitis (VKC), atopic keratoconjunctivitis (AKC), DED and healing autoimmune conjunctivitis are chronic and/or severe conditions of the ocular surface. These latter types of chronicsevere conditions are characterised by the possibility of corneal damage, which may lead to loss of vision. Although recent studies have cast light on the biological mechanisms underlying acute and chronic ocular surface inflammation, identifying new potential therapeutic targets, there is still a lack of adequate treatment for more severe (chronic) ocular surface diseases.

\section{II.3.1. Dry Eye Disease}

DED is one of the most prevalent ocular surface disorders. According to the Dry Eye Workshop (DEWS), DED is "a multifactorial disease of the tears and ocular surface that results in symptoms of discomfort, visual disturbance, and tear film instability with potential damage to the ocular surface, accompanied by increased osmolarity of the tear film and inflammation of the ocular surface". ${ }^{4}$

DED is recognised as a chronic eye disease due to inflammation and dysfunction of the LFU. These events cause abnormal tear film composition, leading to an unstable precorneal film layer. When these factors are combined, they produce ocular symptoms that can interfere with activities of normal daily life, such as reading or driving. ${ }^{50}$ Patients with DED experience varying severity of symptoms and commonly refer to intermittent-to-constant eye irritation, pain, photophobia, redness, ocular burning and blurred vision. ${ }^{49}$ Indeed, the impact of the severe form of DED on patients is in the range of a life-threatening cardiac condition. ${ }^{51}$ There is no definitive therapy for DED and it remains one of the leading causes of patient visits to ophthalmologists. ${ }^{52}$

\section{II.3.1.1. Prevalence and risk factors}

The prevalence of DED ranges from $2 \%$ to $14 \%$ of the worldwide population, depending on the definition and the population studied..$^{53-56}$ The estimated prevalence of DED within the Spanish population is $11 \%{ }^{57}$ Although DED increases with age, ${ }^{58}$ prevalence among women is greater than men in all age groups. ${ }^{54,56}$

Several risk factors for the development of DED have been identified repeatedly in epidemiological studies. DED occurs with greater frequency in women, particularly in postmenopausal women because sex hormones influence ocular surface conditions through their effects on tear secretion, meibomian gland function and goblet cell density. ${ }^{59,60}$ Risk factors also involved in the development and impairment of DED 
include contact lens wear, ${ }^{61}$ refractive surgery ${ }^{62}$ and cigarette smoking. ${ }^{63}$ Extended visual tasking during computer use, television watching or prolonged reading can also provoke symptoms of DED. ${ }^{64}$ Adverse environmental conditions like low relative humidity and aircraft cabins can cause and worsen DED. ${ }^{65,66}$ Moreover, DED is also associated with systemic and autoimmune diseases such as diabetes mellitus ${ }^{56}$ and rheumatoid arthritis, ${ }^{67}$ respectively. Other factors that provoke and/or exacerbate DED include chronic use of preserved eye $\operatorname{drops}^{68}$ and systemic drugs that inhibit tear production. ${ }^{69}$

\section{II.3.1.2. Classification for dry eye disease}

Based on aetiological factors that can influence LFU, DED is divided into aqueous tear-deficient dry eye (ADDE) and evaporative dry eye (EDE) ${ }^{49}$

ADDE is characterised by reduced tear secretion and volume due to a failure of the LFU. ADDE is subcategorised into non-Sjögren and Sjögren DED.

Non-Sjögren DED is due to lacrimal dysfunction without signs of systemic autoimmunity, whilst Sjögren DED is an autoimmune process that affects lacrimal, salivary and other exocrine glands in conjunction with other systemic diseases, such as rheumatoid arthritis.

EDE is a DED subset characterised by an excessive evaporation of the tear film from the ocular surface due to an unstable lipid layer, but tear secretion is normal. It is a result of a meibomian gland dysfunction, which is one of the most common causes, along with a low blink rate.

\section{II.3.1.3. Pathophysiology of dry eye}

Although the exact cellular and molecular mechanisms underlying DED are not completely understood, it has been shown that immune-mediated inflammation plays a key role in this disease. ${ }^{70}$

Inflammation in the LFU is the central feature of DED. Tear composition in DED is characterised by decreased levels of epithelial growth factor (EGF) and mucins, ${ }^{71,72}$ and increased concentrations of pro-inflammatory cytokines and activated proteases. ${ }^{73,74}$ Loss of tear film stability leads to disruption of the corneal epithelial barrier function resulting in abnormal signalling from the corneal nerves to the central nervous system, the lacrimal gland and other secretory glands. Corneal nerve abnormalities lead to further ocular surface damage and help perpetuate the vicious inflammatory cycle of DED (Figure 7). 
Inflammation of both the lacrimal gland and conjunctiva has been described in Sjögren and non-Sjögren-associated DED patients. ${ }^{75-78}$ Pro-inflammatory cytokines, for instance IL-1 $\alpha, I L-1 \beta, I L-6$, TNF- $\alpha$ and TGF- $\beta 1$, are increased in tears and/or in conjunctival epithelium of DED patients, compared to healthy subjects. ${ }^{65,71,79-81}$ Several additional cytokines have been isolated from the ocular surface of patients with DED, including IL-2, IL-4, IL-5, IL-10 and IFN- . $^{82}$

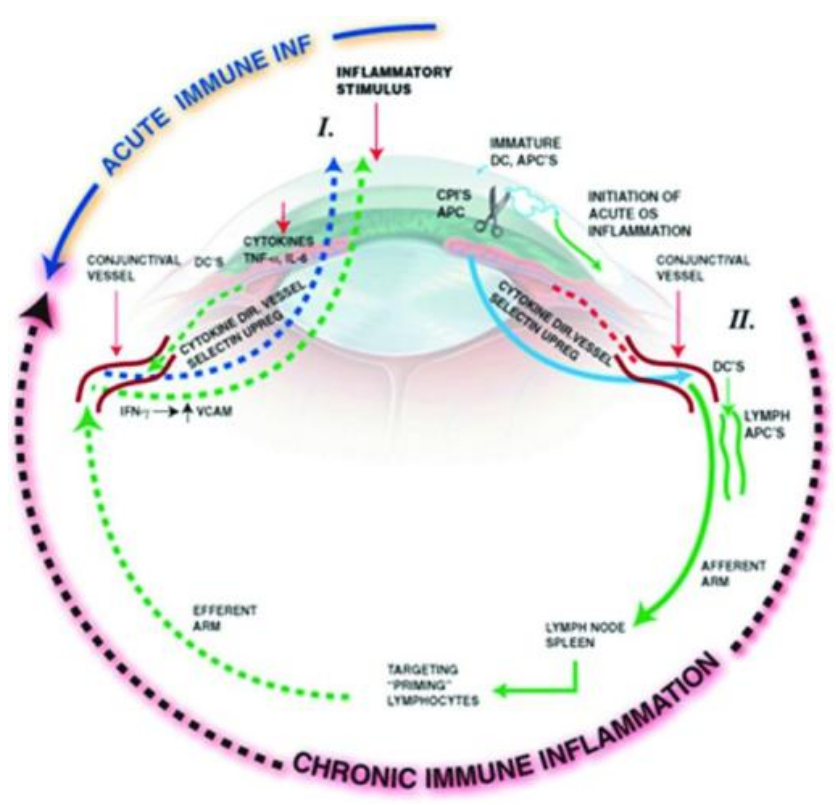

Figure 7. Inflammation circle of chronic DED. Adapted from Stern et al. 2013. ${ }^{10}$

Chemokines are cytokines that regulate the chemotaxis (directed migration) of immune cells. The chemokine IL-8/CXCL8 (a neutrophil chemoattractant involved in the innate immune response) has been identified in the tears and conjunctiva of patients with DED. ${ }^{80}$ The closely related chemokines CXCL9, IP-10/CXCL10 and CXCL11 are also elevated in the tear film and ocular surface of patients with DED. ${ }^{80,81}$ These latter chemokines are produced in response to IFN- $\mathrm{y}$, and function as $\mathrm{T}$ cell chemoattractants.

The release of these cytokines and chemokines from the diseased ocular surface epithelia indicate that lymphocytes play a key role in the immunopathogenesis of chronic DED. CD4 ${ }^{+} \mathrm{T}$ cells infiltration into the conjunctiva and lacrimal glands have been found in patients with DED. ${ }^{4,10,79}$ Moreover, activated CD4 ${ }^{+}$T cells are also found in the ocular surface of DED; ${ }^{77,78}$ along with $\mathrm{CD} 4^{+} \mathrm{T}$ cell-derived cytokines such as IFN$\mathrm{Y}$, which can interfere with conjunctival epithelial differentiation and produce apoptosis in mucosal epithelia. ${ }^{83}$ In addition, CAMs promote the infiltration of immune cells into the ocular surface of patients with DED. Elevated levels of intercellular adhesion 
molecule (ICAM)-1 (that binds to lymphocyte function-associated antigen 1) and vascular CAM (VCAM)-1 (expressed by the vascular endothelium and binds to immune cell-expressed integrin $\alpha 4 \beta 1$ ) have been identified in the conjunctiva and lacrimal gland of patients with DED. 84,85

All of these events show that inflammation of the LFU is one the biological processes underlying DED.

\section{II.3.1.4. Experimental murine model of dry eye}

Animal models have contributed greatly to advanced understanding of DED and have helped to develop better therapeutic strategies. For studying DED signs, dogs, (less frequently) cats, and (rarely) horses may naturally develop DED, presenting decreased tear secretion and ocular surface changes. Indeed, immune-mediated destruction of the lacrimal gland tissues is a frequent cause of DED in dogs. There are also rabbit DED models, mostly focused on lacrimal gland inflammatory alterations. Nevertheless, murine models are the most commonly used to study autoimmune mechanisms. ${ }^{86}$ One of the most important in-vivo models of DED is the so-called environmental model, first reported by Dursun et al. ${ }^{87}$ (Figure 8 ). In this model, mice are exposed to desiccating stress (DS-constant airflow and low humidity), combined with the administration of the anticholinergic scopolamine in order to induce DED through different primary pathogenic mechanisms. ${ }^{88} \mathrm{DS}$ are produced using either an air blower or a controlled-environment chamber that allows regulation of environmental conditions, ${ }^{89,90}$ similar to that used for human studies in which temperature, humidity and airflow can be controlled. ${ }^{65,91}$ This in-vivo model has demonstrated that mice exposed to DS develop clinical and histopathological similarities to the human disease, such as lacrimal gland dysfunction, goblet cell loss, increased cytokine/chemokine production, and squamous metaplasia and apoptosis on the ocular surface, including a robust $\mathrm{CD}^{+} \mathrm{T}$ cell infiltrate into the LFU. ${ }^{87,92-96}$

The most important evidence that confirmed DED is an immune-based disease was presented by Dr. Stern and colleagues, using an adoptive transfer model after the DS model. ${ }^{94}$ In this adoptive transfer model, $\mathrm{CD} 4^{+} \mathrm{T}$ cells are isolated from the cervical lymph nodes (CLNs) and/or spleen of mice exposed to DS, which are then transferred to athymic T-cell-deficient nude-recipient mice (Figure 8). As a result, CD4 ${ }^{+} \mathrm{T}$ cells are localised within the ocular surface and lacrimal gland tissues of recipient mice where they mediate full-blown DED by increasing cytokine levels, and decreasing tear production, tear turnover and goblet cells density; even though recipient mice have never been exposed to DS. Furthermore, inflammation is restricted to the lacrimal 
gland and ocular surface in recipient mice. This adoptive transfer model demonstrates that $\mathrm{CD}^{+} \mathrm{T}$ cells isolated from mice exposed to DS are sufficient to cause inflammatory disease in T-cell-deficient nude-recipient mice.

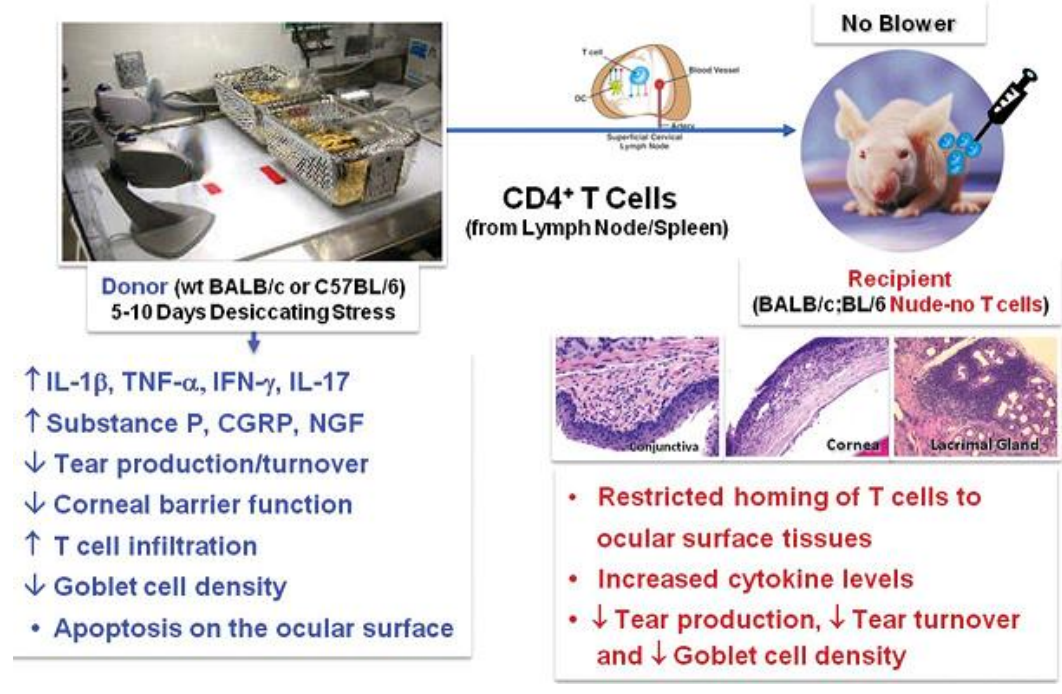

Figure 8. Experimental DED model and adoptive transfer model. Adapted from Stern et al. 2013. ${ }^{10}$

Recent evidence points towards the aetiology of DED being driven by autoantigens. Kallikrein 13 (Klk13), an autoantigen identified in a murine DED model, ${ }^{97}$ is found in cornea, conjunctiva and lacrimal gland after DS. ${ }^{98}$ Expression of this autoantigen corresponds with the development of autoantibodies specific for Klk13. Passive transfer of anti-Klk13 autoantibody to nude mice is sufficient to induce ocular surface inflammation through mechanisms such as a pro-inflammatory cytokine response (IL-6, IL-12, IL-17, TNF- $\alpha$ and IFN- $y$ ) and infiltration of neutrophils within ocular surface tissue. These events suggest that autoantibodies are involved in the pathogenesis of experimental DED, but from the perspective of human disease the specific self-antigens are still widely unknown among organ-specific autoimmune diseases. ${ }^{70}$

In addition, APCs are necessary to naïve $\mathrm{T}$ cell activation and differentiation and it has been demonstrated that APCs play a role in regulating T cells in DS model. Accumulation of mature DCs correlates with $\mathrm{CD4}^{+} \mathrm{T}$ cell activation in CLNs after exposure to DS. ${ }^{99}$ APC depletion by liposome-encapsulated clodronate inhibits the generation of autoreactive $\mathrm{CD}^{+} \mathrm{T}$ cells and preserves goblet cells within the conjunctiva. ${ }^{99}$ Moreover, lymphadenectomised mice exposed to DS also display less infiltrating $\mathrm{CD}^{+} \mathrm{T}$ cells and higher goblet cell density. These events corroborate the hypothesis that activation of autoreactive $\mathrm{CD} 4^{+} \mathrm{T}$ cells occurs in the draining CLNs via cell-to-cell contact with ocular surface-derived APCs. ${ }^{99}$ 
Taken together, all these studies indicate that a murine model of DED allows us to understand the pathophysiological mechanisms involved in the development of DED, being a suitable in-vivo model for testing new anti-inflammatory compounds.

\section{II.3.1.5. Treatment of dry eye}

Advances in the comprehension of the mechanisms involved in DED have led to developments of a wide range of therapeutic strategies. Traditionally, DED has been linked to tear evaporation and the aim has been to restore physiological tear function. Artificial tears are still the most common and widely used therapy for the treatment of DED patients. They are artificial lubricants containing electrolytes and surfactants, in an isotonic or hypotonic buffered solution. Although they temporally improve symptoms, such as eye irritation and blurred vision, they do not have any effect on the inflammatory cascade involved in DED. A major advance in DED therapy has been the incorporation of anti-inflammatory therapies. Currently, topical corticosteroids, oral tetracyclines, essential fatty acids (EFAs) and topical cyclosporine are routinely used in the management of the inflammatory process involved in DED. ${ }^{100}$

- Topical corticosteroids, such as methylprednisolone, are well known for their effects on the inflammatory cascade, specifically the blockade of cyclo-oxygenases (COX) and production of prostanoids from arachidonic acid. Corticosteroids have been shown to reduce inflammation and provide relief of symptoms in Sjögren DED patients. ${ }^{101,102}$ However, chronic administration of corticosteroids has side-effects including increasing intraocular pressure and cataract formation. ${ }^{103-105}$

- Tetracyclines are antibiotics that interfere with protein synthesis of many bacteria, mycoplasma and chlamydia. These bacteria can release lipases that metabolise meibomian lipids into diglycerides and free fatty acids, which are highly toxic to the ocular surface epithelium in ADDE. ${ }^{106}$ Although tetracyclines are considered as antibiotics, they also have several anti-inflammatory properties including inhibition of MMP synthesis and activity, and decrease of IL-1 in corneal epithelial cells. ${ }^{107,108}$ Side-effects associated with tetracyclines include gastrointestinal upset (i.e. gastritis), yeast infections and photosensitivity. ${ }^{109}$

- EFAs are the precursors of eicosanoids (prostaglandins, prostacyclins, thromboxanes, and leukotrienes) that modulate immune response. Recent studies have shown significant improvement in ocular irritation symptoms and decreased ocular surface staining by EFAs such as linolenic acid and $\mathrm{y}$-linolenic acid, administered orally. ${ }^{110}$ Evidence also suggests that supplementation with omega-3 
and -6 EFAs may be beneficial in the treatment and prevention of DED. ${ }^{111,112}$ However, more evidence is needed to identify the most efficacious forms and doses of EFAs, and their effects on the ocular surface.

- Cyclosporine A, a natural-occurring compound, is commonly used to treat autoimmune diseases and as an immunosuppressant to control transplant rejection. Topical cyclosporine A was approved by the Food and Drug Administration (FDA) in December 2002, and became commercially available in April 2003 as Restasis ${ }^{\mathrm{TM}}$ (0.05\% cyclosporine A, Allegan Inc., Irvine, California, USA) for treating inflammation in DED. Cyclosporine A relieves the symptoms and signs associated with DED. Patients suffering DED treated with topical cyclosporine A show a decreased in IL-6 levels, ${ }^{113}$ reduced human leukocyte antigen (HLA)-DR and CD11a (a marker of activated $\mathrm{T}$ cells) in the conjunctival epithelium ${ }^{77}$ and also show increased conjunctival goblet cell density after 6 months of treatment. ${ }^{114,115}$ Not only does cyclosporine A decrease inflammation in the ocular surface, but it can also inhibit apoptosis involved in DED. ${ }^{116}$ Some side-effects are related to cyclosporine A use. For example, oral and intravenous administration of cyclosporine $A$ can produce serious side-effects (i.e. hypertension and nephrotoxicity), but topical cyclosporine A for DED treatment has low systemic absorption, and none of those side-effects have been reported. In addition, topical cyclosporine A takes several months to achieve its full effect. Activated lymphocytes live in the human body for about 110 days and cyclosporine A cannot "turn off" activated $T$ cells like corticosteroids do, because it prevents further $\mathrm{T}$ cell activation. Therefore, it takes at least 110 days to reduce levels of all the activated lymphocytes in the inflamed tissues. As a result, several months of topical administration of cyclosporine $A$ is necessary before reaching improvements in ocular surface disease.

\section{II.3.2. Ocular Allergy}

Ocular allergy is a localised allergic condition that is observed as the only (or dominant) presentation of an allergic sensitisation. Ocular allergies are encountered daily in the physician's office; being one of the most common ocular conditions observed in clinical practice with a prevalence of between $20 \%$ and $40 \%{ }^{117}$ It is estimated that ocular symptoms are present in $40 \%-60 \%$ of allergic patients. ${ }^{118}$ Allergic eye disorders primarily affect the conjunctiva, cornea and eyelids, and can be acute or chronic. Acute allergy is IgE-mediated mast cell degranulation, whilst chronic allergies are mediated by $T$ cells, eosinophils and continuous activation of mast cells. ${ }^{19,120}$ The acute form involves transient symptoms of itching, tearing and swelling, while chronic 
allergies exhibit symptoms such as severe pain and visual disturbances. In recent years, new drug candidates have been developed from the understanding of the pathophysiology of ocular allergies. However, there is not yet an effective treatment for chronic conditions, which may lead to corneal opacity and vascularisation.

\section{II.3.2.1. Allergic eye diseases}

Allergic eye diseases can be divided into the following types: allergic conjunctivitis (seasonal-SAC- and perennial-PAC-), VKC, AKC and giant papillary conjunctivitis (GPC). ${ }^{121}$ SAC and PAC display a mild presentation, whereas VKC, AKC and GPC are more severe.

SAC and PAC are the most common forms of ocular allergies, representing $15 \%-20 \%$ of the population, ${ }^{122}$ and they affect the patient's quality of life, having a significant socioeconomic impact. ${ }^{123,124}$ Comparing both conditions, the prevalence of PAC had been reported to be lower than that of SAC $(3.5: 10,000) .{ }^{125}$ Clinical symptoms and signs in both diseases are the same: tearing, itching, redness, chemosis and swelling of the conjunctiva; whilst the cornea is unaffected in SAC and PAC. Symptoms of SAC are generally more severe in comparison to PAC. Both SAC and PAC involve IgE-mediated mast cell activation. They are often related to known allergens, such as grass and tree pollen. As allergens involved in SAC are seasonal, every symptom of SAC disappears at the end of season. However, allergens involved in PAC are continuous rather than seasonal, being a chronic condition. Eosinophils and neutrophils have been detected in the conjunctival tissues of patients with SAC or PAC. Additionally, $T$ cells have also been detected in patients suffering PAC. ${ }^{126}$

VKC is a bilateral chronic condition. It is a persistent and severe form of ocular allergic, mainly affecting children and adolescents. ${ }^{127}$ VKC is more common in warm climates: the estimated overall prevalence in Europe is $3.2 / 10000$, with a higher prevalence observed in Italy $(27.8 / 10000)$ where the climate is warmer; and a lower prevalence is found in Norway (1.9/10000). ${ }^{128}$ There are three clinical forms of VKC: palpebral, limbal and mixed. Both the conjunctiva and cornea are involved in VKC. Symptoms include ocular redness, itching, tearing and swelling. Patients can also have photophobia. However, the most characteristic sign are giant papillae on the upper tarsal conjunctiva in which IL-4 and IL-13 are involved by inducing the proliferation of conjunctival fibroblasts. ${ }^{119,129}$ Complications include conjunctival fibrosis and superficial punctate keratopathy. Although VCK is mainly mediated by Th2 lymphocytes, cells of innate immunity such as eosinophils, mast cells and neutrophils become activated, infiltrating the conjunctival subepithelial and stromal tissues. ${ }^{130}$ In addition, IgE- 
mediated sensitisation is found in $50 \%$ of patients, ${ }^{131}$ and high values of plasma histamine have been measured in VKC, but not in SAC, supporting the concept that VKC is a chronic disease. ${ }^{132}$

$A K C$ is the most severe of chronic allergic eye diseases. It has been reported to affect between $25 \%-40 \%$ of patients suffering from atopic dermatitis, but its real prevalence is unknown because the prevalence of its dermatological counterpart, atopic dermatitis, seems to be growing. ${ }^{133}$ Although there is no agreement about the diagnostic criteria for AKC, it may be defined as a chronic ocular surface noninfectious, inflammatory condition associated with other atopic conditions, occurring at any time point independently of its degree of severity, as well as corneal involvement at some time in the course of the disease. ${ }^{133,134}$ Symptoms of AKC are characterised by itching, redness, blurred vision, photophobia and pain. Signs of AKC depend on the severity of this disease and may range from anterior blepharitis, conjunctival hyperaemia and superficial punctuate keratitis (in mild AKC) to anterior and posterior blepharitis, symblepharon and corneal neovascularisation (in severe AKC). ${ }^{133}$ Regarding its molecular mechanism, $\mathrm{CD}^{+}{ }^{+} \mathrm{T}$ cells, eosinophils, and neutrophils are the predominant cell types that infiltrate the conjunctival tissue. ${ }^{135}$ In addition, high levels of multiple cytokine and chemokine profiles have been detected in tears, suggesting that both Th1 and Th2 responses may be activated. ${ }^{136-139}$

GPC is an inflammatory condition characterised by papillary hypertrophy of the superior tarsal conjunctiva and its appearance is similar to vernal conjunctivitis, without corneal involvement. ${ }^{140}$ GPC is most frequently caused by ocular prostheses, postoperative sutures and contact lenses. ${ }^{141}$ The conjunctival papillary changes resolve when these irritating stimuli are removed.

\section{II.3.2.2. Pathophysiology of ocular allergy}

Several cells, such as mast cells, eosinophils, neutrophils or APCs are involved in allergic eye diseases. The clinical response in all forms of allergic eye disease is mainly due to mast cell activation; either via lgE cross-linkage or mediated by $\mathrm{T}$ cells, leading to release of inflammatory molecules and cytokines to the ocular surface. $^{68,120,142}$

\section{II.3.2.2.1. IgE mediated-hypersensitivity in ocular allergy}

Ocular allergic inflammation is associated with IgE-mediated mast cell activation (type I hypersensitivity reaction) in conjunctival tissue. There are two phases in specific 
IgE-mediated conjunctival mast cell activation, early and late, which involve different kinds of immune cells and inflammatory molecules.

The early phase clinically lasts between 20 and 30 minutes, as demonstrated by specific conjunctival allergen challenge. ${ }^{143}$ This phase occurs when allergens bind to the high affinity $\operatorname{lgE}$ receptor ( $\mathrm{Fc \varepsilon RI}$ ) at the surface of mast cells, thus inducing cell degranulation. It leads to increased levels of histamine, tryptase and inflammatory mediators such as prostaglandin D2 and leukotrienes in tears, ${ }^{142}$ which trigger the recruitment of eosinophils and basophils, producing clinical symptoms. These inflammatory factors initiate the late phase that occurs 4-6 $\mathrm{h}$ after the early phase response. It is a process that intensifies the allergic response and heightens the inflammatory process. This intensification is the major cause of ocular surface damage in ocular allergic diseases such as keratitis, limbal infiltration and corneal ulcers. The late phase is characterised by $T$ lymphocyte activation, production of Th2-lymphocyte type cytokines and infiltration of inflammatory cells such as neutrophils, lymphocytes, basophils and eosinophils in the conjunctival mucosa. ${ }^{119}$

Mast cells are granulocytic cells located mainly in the substantia propria in SAC and PAC and in the normal conjunctiva, whilst mast cells in VKC and AKC are in both the substantia propria and epithelium. Mast cells have not been found in the cornea of normal eyes. There are two forms of conjunctival mast cells, characterised on the basis of their tryptase and chymase expressions: the tryptase-positive and chymase-positive mast cells (MCTC) and the tryptase-positive and chymase-negative mast cells (MCT). More than $95 \%$ of mast cells found in the substantia propria of the normal human conjunctiva are MCTC. ${ }^{144,145}$ Although MCTC are increased in subjects with vernal conjunctivitis and allergic conjunctivitis, MCT are only increased in the epithelium of the latter condition. ${ }^{144}$

Mast cell granules constitutively contain various enzymes and pro-inflammatory mediators. One of the most important mediators of anaphylactic reactions that is secreted by mast cells and basophils is histamine (2-[4-imidazolyl]ethylamine). Histamine is an amine that has a major role in the innate immune response in allergic inflammation because it produces vasodilatation (increasing vascular permeability), and it promotes DC migration ${ }^{146}$ and maturation, ${ }^{147}$ along with other biological effects, such as gastric acid secretion. ${ }^{148}$ At the ocular surface, histamine is highly secreted by mast cells after lgE cross-linking in the conjunctiva, but is rapidly degraded by tear histaminase enzymes. It exerts its effects by binding to its four receptors $(H 1 R, H 2 R$, $\mathrm{H} 3 \mathrm{R}$ and $\mathrm{H} 4 \mathrm{R}$ ), which all are present in the human normal conjunctiva. Clinically, tearing, itching, hyperaemia and lid swelling are related to histamine secretion. 
Mast cells also release IL-4, IL-5, L-6, IL-8/CXCL8, IL-13 and TNF- $\alpha,{ }^{142,149}$ but both types of mast cells are heterogeneous with respect to cytokine storage. ${ }^{150}$ For example, IL-4 and IL-13 preferentially localise to the MCTC, and IL-5 and IL-6 to the MCT phenotype. ${ }^{150}$ This indicates that, depending on protease phenotype, mast cells have different effects. For example, IL-4 that is secreted by MCTC, plays a pivotal role in allergy promoting $T$ cell growth, Th2 differentiation and IgE production; ${ }^{150}$ whilst IL-6, a pro-inflammatory cytokine secreted by MCT, promotes Th2 differentiation and simultaneously inhibits Th1 polarisation through two independent molecular mechanisms. ${ }^{151}$

Not only do these molecules have effects on immune system cells but they also stimulate epithelial cell secretion. It is known that both corneal and conjunctival epithelial cells participate in the immune response via expression of surface antigens and adhesion molecules, and secretion of cytokines and chemokines. ${ }^{152}$ For example, normal conjunctival tissue express several cytokines and chemokines such as IL-6, IL8/CXCL8, RANTES/CCL5 and TNF- $\alpha$, among others, ${ }^{153}$ but do not express ICAM-1 and HLA-DR, whilst these latter two are found in patients suffering allergic conjunctivitis. $^{68,153}$ In addition, epithelial cytokines including IL-3, IL-8/CXCL8, granulocyte-macrophage colony-stimulating factor (GM-CSF) and RANTES/CCL5 are upregulated in allergic diseases. ${ }^{153}$ Conjunctival and corneal epithelia are also stimulated by mast cell-secreted cytokines, leading to the production of more proinflammatory cytokines and chemokines. For example, TNF- $\alpha$ stimulates up-regulation of IL-6 and IL-8/CXCL8 by both conjunctival and corneal epithelial cells. ${ }^{153,154}$ In addition, conjunctival fibroblasts contribute to cytokine and chemokine production. Stimulation of conjunctival fibroblasts with TNF- $\alpha$, alone or in combination with either IL-4 or IL-13, results in upregulation of IL-6, IL-8/CXCL8, eotaxin, monocyte chemoattractant protein (MCP)-1/CCL2, RANTES/CCL5 and IP-10/CXCL10. ${ }^{139}$ These studies therefore suggest both conjunctival epithelial cells and fibroblasts play a key role in allergic eye diseases.

\section{II.3.2.2.2. T lymphocytes mediated-hypersensitivity in ocular allergy}

T cells are involved in the type IV hypersensitivity response, also known as cellmediated immunity, which occurs in chronic allergic conditions. ${ }^{155}$ Type IV reactions involve two phases: sensitisation and elicitation. The sensitisation phase occurs when APC processes antigen-MHC class II complex to T cells, resulting in the differentiation of $\mathrm{CD}^{+} \mathrm{T}$ cells into effector $\mathrm{CD}^{+}{ }^{+} \mathrm{T}$ cells and memory $\mathrm{T}$ cells. The elicitation phase occurs when memory $\mathrm{T}$ cells recognise the antigen peptide together with $\mathrm{MHC}$ class II 
molecules on an APC. This interaction stimulates memory $\mathrm{T}$ cells to proliferate and release cytokines.

This hypersensitivity response is due to interaction of antigens with Th1 and Th2 lymphocyte subsets. Both Th1 and Th2 activation are involved in the immunological response of VKC and AKC, but VKC has a predominantly Th2 profile, whilst AKC has a shift towards a Th1-like profile. ${ }^{120}$ Th17 and Treg lymphocyte subsets also have a role in the pathogenesis of conjunctivitis, but their roles are not completely understood. ${ }^{156}$

Recent findings suggest that innate $T$ cells, such as NK cells and $y / \delta T$ cells, also have an important role in allergic diseases. ${ }^{157-159}$ NK cells can produce IFN- $\gamma$, and IL-4, IL5 and IL-13 cytokines, inhibiting or stimulating the allergic response, along with their known cytotoxic activity. In VKC, NK cell numbers decrease in the blood and increase in the conjunctiva. ${ }^{157}$ This indicates a potential role for NK cells in the regulation of allergic reactions. ${ }^{157}$

\section{II.3.2.3. Treatment for ocular allergy}

Several treatments, both systemic and topical, are currently available to manage the signs and symptoms of allergic conjunctivitis. Topical lubricants, such as artificial tears, are usually applied for rinsing antigens from the eye. However, they only provide temporary relief of symptoms and do not have any effect on the inflammatory process associated with allergy.

Drugs for treating ocular allergies belong to different pharmacological classes: antihistamines, mast cell stabilisers, dual acting agents, vasoconstrictors, non-steroidal anti-inflammatory agents, corticoids and immunomodulatory agents.

- $\quad$ Topical antihistamine drugs are the most preferred treatment for ocular allergies. They are classified into first and second generation. The first generation $\mathrm{H} 1$ antagonists have a long safety record. Although they have rapid onset, their effects disappear very quickly. In addition, they are known for a burning sensation upon instillation and their limited potency. ${ }^{160}$ In contrast, the second generation of $\mathrm{H} 1$ antagonists are non-sedating and have a longer duration of action (4-6 h), but they also have side-effects such as burning, redness and/or stinging upon instillation. Oral antihistamines are also available but when symptoms are primarily ocular, topical antihistamines should be used.

- Mast cell stabilisers inhibit degranulation resulting from the activation of $\lg \mathrm{E}$ receptors. ${ }^{161}$ Thus, they are effective in both acute and chronic allergic eye diseases. They have few side-effects including burning and stinging, but they all 
require a preloading period and frequent instillation every day, which sometimes results in poor compliance. ${ }^{162}$

- Dual-action agents, which are mast cell stabilisers with antihistamine action, provide rapid relief from symptoms. ${ }^{163}$ However, some transient and mild sideeffects appear after instillation, such as stinging, burning and a transient bitter taste. $^{164}$

- Vasoconstrictors are alpha-adrenergic agonists approved topically for relief of conjunctival redness, but have poor efficacy on other symptoms. They are also non-specific, without any effect on the allergic reaction. Adverse effects of topical vasoconstrictors include burning and stinging upon instillation, as well as mydriasis and rebound hyperaemia or conjunctivitis medicamentosa with long-term use. ${ }^{165}$

- Non-steroidal anti-inflammatory drugs partially inhibit events that follow degranulation, but they are not the first-line therapy for ocular allergies. They can cause discomfort upon instillation (stinging and burning sensation) and should be used with caution in patients with intolerance, because corneal melting and perforation have been described. ${ }^{166}$

- Topical corticosteroids do not have any effect on mast cells and further events, but they may modulate the mast cell response by inhibiting cytokine production, and activation/recruitment of inflammatory cells. ${ }^{167}$ They have side-effects from chronic application, such as increasing intraocular pressure and cataract formation, delaying wound healing and increasing viral and bacterial infections. ${ }^{168}$

In addition, it is known that cyclosporine A reduces ocular surface inflammation and inhibits histamine release from mast cells and basophils. ${ }^{169,170}$ Therefore, it has been suggested that topical cyclosporine A may be also a useful therapy for the treatment of severe VKC and AKC. ${ }^{134}$

\section{II.4. Natural Compounds: Polyphenols}

The medicinal use of compounds that come from natural sources, such as plants and fruit, has had a great contribution to human health care. Natural products have played a key role in medicine, being for several centuries the sole means to treat injuries and diseases. For example, from our earliest ancestors who relieve their pain chewing on herbs, or the ancient civilisations such as Greeks or Chinese that provided written evidence of using plants for the treatment of a wide variety of diseases, ${ }^{171,172}$ to current anticancer drugs, such as paclitaxel or camptothecin. ${ }^{173}$ The earliest known 
record, written on clay tablets, is from Mesopotamia (2600 BC), describing the use of natural compounds for the treatment of coughs and colds. ${ }^{174}$ Yet it was not until the $19^{\text {th }}$ century, when active compounds from various medicinal plants were isolated and characterised, ${ }^{175}$ that a series of natural products became very important clinical agents, that are still in use today. These compounds include quinine, morphine and codeine ${ }^{175,176}$ as well as antibacterial compounds from micro-organisms such as penicillin, cephalosporin and streptomycin. ${ }^{172}$ In addition, new technologies have provided new tools to purify and determine natural product structures, allowing development of natural compound-based drugs.

The World Health Organisation (WHO) pointed out that up to $80 \%$ of people in developing countries use traditional, complementary or alternative medicine as part of primary health care. ${ }^{177}$ According to Newman, $60 \%$ of currently available drugs have been either directly or indirectly derived from natural compounds. ${ }^{178}$ About $25 \%$ of prescribed drugs worldwide come from plants and 121 such active compounds are in use. ${ }^{179}$ Moreover, $11 \%$ of the drugs considered essential medicines by the WHO are exclusively from plant origin or natural compound-based synthetic drugs. ${ }^{180}$ Therefore it is a matter of great scientific, economic and medical interest to understand and analyse how natural products are beneficial for human health.

All natural products can be classified according to their source: marine, plant, animal and the microbial world. Focusing on plant sources, polyphenols are probably the most investigated naturally-occurring compounds.

Polyphenols are normally produced by plants for their antibiotic and antifungicidal features. ${ }^{181}$ Structurally, polyphenols can be classified into different groups according to the number of phenol rings that they contain, and to the structural elements that bind these rings to one another. Distinctions are thus made between lignans, phenolic acids, stilbenes and flavonoids ${ }^{182}$ (Figure 9). Among all these polyphenol groups, interest focuses on stilbenes and flavonoids. 


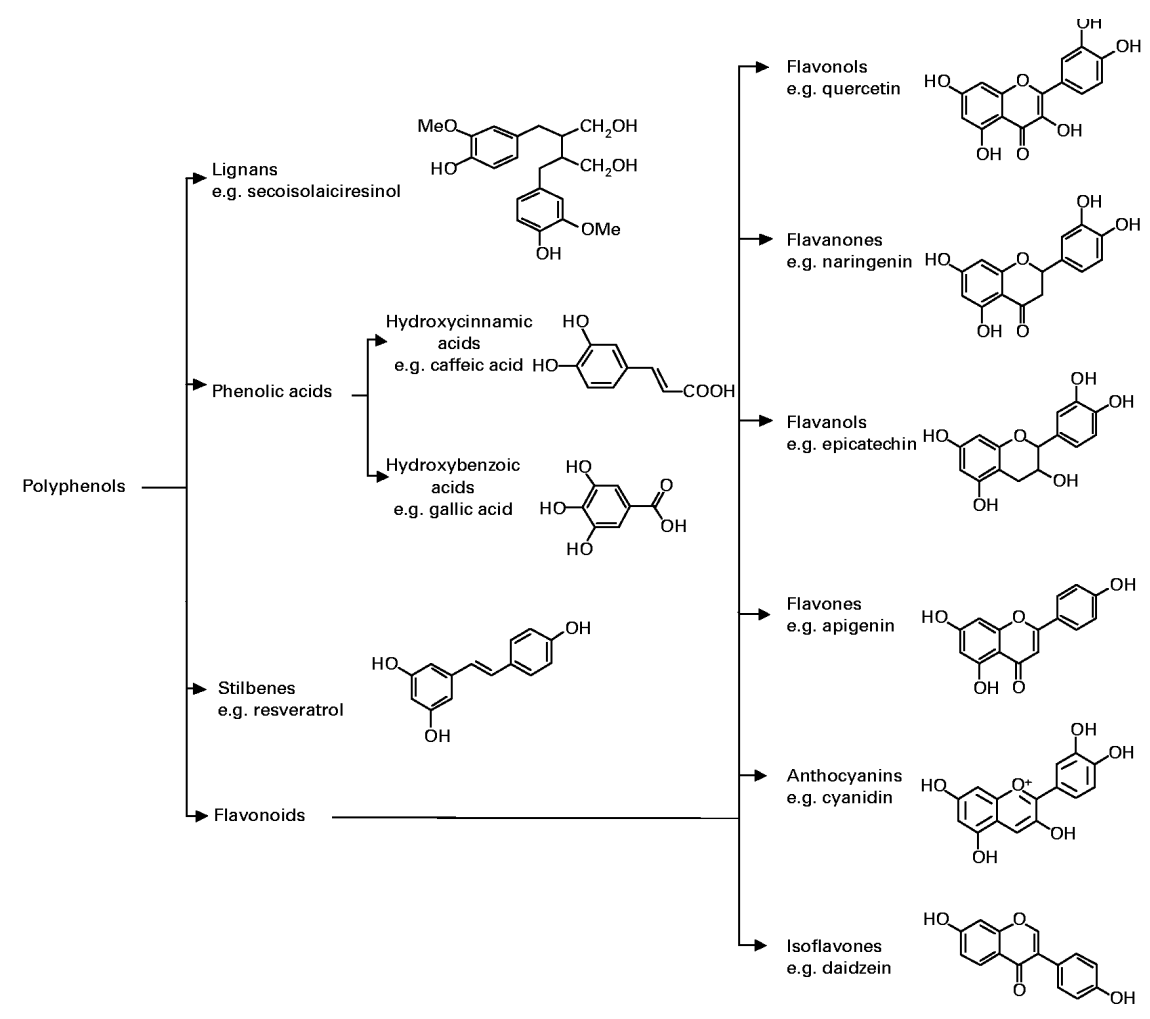

Figure 9. Classification and chemical structure of major classes of polyphenols.

Adapted from Spencer et al. 2008. ${ }^{182}$

\section{II.4.1. Stilbenes}

Stilbenes are a small family of molecules found in a wide range of plants and fruits such as berries and grapes, but they are found in low quantities in the human diet. The name for stilbenes was derived from the Greek word stilbos that means "shining". Stilbenes are often referred to as phytoalexins, due to their protective action upon secretion. ${ }^{183}$ These metabolites act as protective agents to defend the plant against microbial attack, excessive ultraviolet (UV) light exposure and diseases. ${ }^{184}$ Upon environmental threat, the plant host activates the phenylpropanoid pathway and as a consequence stilbene structures are secreted.

Stilbenes are molecules characterised by a 1,2-diphenylethylene backbone (two phenolic rings connected by a two-carbon methylene bridge) in their chemical structures. These compounds have generated much scientific research in their potential clinical applications in the treatment of diseases, because they have shown potent anti-inflammatory, anti-cancer and chemoprotective effects, among others. ${ }^{185-187}$ One of the most popular and well known stilbene is resveratrol (RES). 


\section{II.4.1.1. Resveratrol}

RES (5-[2-(4-hydroxyphenyl)ethenyl]benzene-1,3-diol; molecular weight = $228.25 \mathrm{~g} / \mathrm{mol}$; CAS 501-30-0; Figure 10) is a stilbene-based compound that was first isolated in 1940 by Michio Takaoka from the roots of white hellebore (veratrum grandiflorum). ${ }^{188}$ It is produced by plants to protect them against fungal infections but RES is also produced under environmental stress, such as UV radiation. ${ }^{189}$ Although RES is not widely distributed throughout the plant kingdom, it is mainly found in berries and in the skin and seeds of grapes, including red wine.

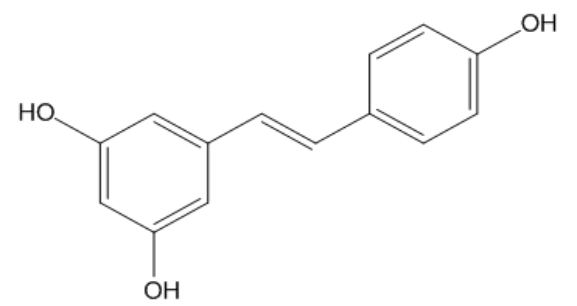

Figure 10. Chemical structure of trans-resveratrol

RES consists of two aromatic rings bridged by ethylene and three hydroxyl groups are attached to the carbon atoms of aromatic rings. There are two isoforms of RES, trans and cis diastereoisomers, due to a double bond in its chemical structure. Although cis-RES is also a natural product found in plants, the name of "resveratrol" commonly refers to trans-RES. Both diastereoisomers are commercially available as cis-isomerisation occurs when the trans-isoform is exposed to UV light and high $\mathrm{pH},{ }^{190,191}$ and because trans-isoform is an extremely photosensitive compound. ${ }^{190}$ Nevertheless, the cis-isomer is less stable in the solid form and less biologically effective ${ }^{192}$ than the trans-isomer, probably due to its non-planar conformation. ${ }^{193}$ In this thesis, the term RES will always refer to the trans-isomer.

RES is a white powder that is soluble in ethanol $(\mathrm{EtOH} ; 50 \mathrm{mg} / \mathrm{mL})$ and dimethyl sulfoxide (DMSO; $16 \mathrm{mg} / \mathrm{mL}$ ), but is poorly soluble in water $(\sim 0.07 \mathrm{mg} / \mathrm{mL}$ ). Nevertheless, RES is a lipophilic molecule and it has high membrane permeability. According to the biopharmaceutical classification system guidance by the FDA, which classifies compounds based on their solubility and permeability, ${ }^{194}$ RES may be considered as a class-II compound (high permeability, low solubility).

Several studies have been carried out to elucidate the pharmacokinetics of RES. For example, Amri et al. summarised the in-vivo fate of RES following oral administration from data obtained in in-vitro cell cultures, ex-vivo cell isolation and invivo experiments, in animals and humans. ${ }^{195}$ When RES is orally administered, intestine absorbs 70 \% RES as a result of a rapid passive diffusion process. RES is 
metabolised by enterocytes forming glucuroconjugates and sulfoconjugates that lead to a maximum plasma concentration between $30 \mathrm{~min}$ and $1.5 \mathrm{~h}$ after intake, depending on the dose administered. ${ }^{196,197}$

The popularity of RES comes from the so-called "French paradox", an epidemiological observation that confirms an inverse correlation between high-fat diet and low incidence of coronary heart disease in some southern regions of France, due to red wine consumption, ${ }^{198}$ meaning RES has cardioprotective properties. However, it was not until 1997, when Jang et al. ${ }^{199}$ reported for the first time the anticancer potential of RES, that the scientific community became more interested in this compound. Since then, there has been an exponential growth of literature about its biological properties related to the number of entries (articles) in PubMed (Medline) published (see Figure 11).

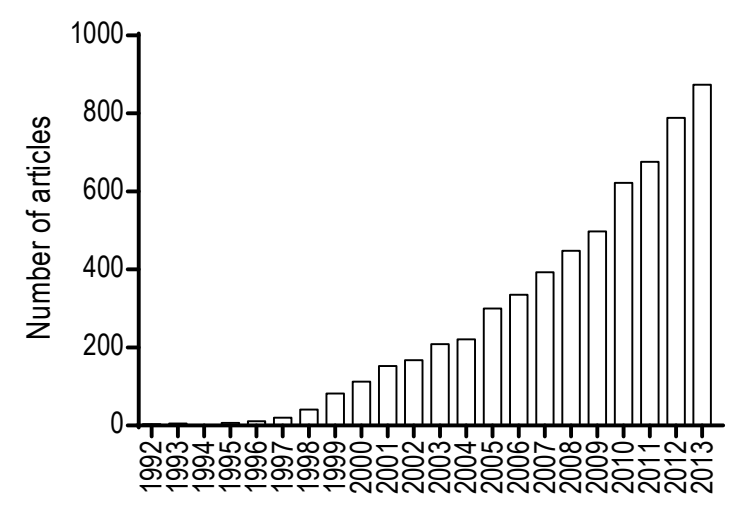

Figure 11. The number of entries (articles) in PubMed published every year using "resveratrol" as keyword.

\section{II.4.1.1.1. Biological properties}

RES has several biological properties as a neuroprotective, cardioprotective and anticarcinogenic compound, ${ }^{199-202}$ but it is also well known as an antioxidant. RES is an effective scavenger of free radicals (reactive oxygen species -ROS- and reactive nitrogen species -RNS-) $)^{203}$ that leads to reduced lipid peroxidation and DNA damage by ROS. ${ }^{204}$ For example, RES is able to inhibit ROS production by polymorphonuclear leukocytes (PMN) stimulated by formyl-methionyl-leucyl-phenyalanine. ${ }^{205}$ In addition, RES protects human lymphocytes activated with hydrogen peroxide $\left(\mathrm{H}_{2} \mathrm{O}_{2}\right)$ against oxidative DNA damage, by increasing the levels of several antioxidant enzymes, such as glutathione peroxidase and glutathione reductase. ${ }^{206}$ RES also extends its antioxidant effect on epithelial cells. Cerqueira et al. found that RES up-regulates glutathione peroxidase and reduces intracellular ROS generation, as well as ICAM-1 
and human beta-defensin-2 expression in lung epithelial cells infected with Pseudomona aeruginosa. ${ }^{207}$ Moreover, Kode et al. demonstrated that RES restores glutathione levels and also quenches ROS in human primary small lung epithelial cells exposed to cigarette smoke. ${ }^{208}$

Although RES has several biological effects, mainly as an antioxidant, the main scientific interest focuses on its anti-inflammatory properties.

\section{II.4.1.1.1.1. Anti-inflammatory properties}

Regarding its anti-inflammatory effects, in-vitro and in-vivo studies have evidenced that RES downregulates the inflammatory response associated with inhibition of activator protein (AP)-1 and the transcription factor nuclear factor (NF)-KB, which is required for the expression of many inflammatory proteins such as GM-CSF, IL-8/CXCL8 and COX-2. ${ }^{209,210}$ Therefore, inhibition of NF-KB may reduce the expression of inflammatory molecules. For example, Manna et al. studied the effect of RES on myeloid cells, lymphoid cells and epithelial cells. They found that RES blocks TNF- $\alpha$-induced activation of NF-KB in a dose- and time-dependent manner, and suppresses TNF- $\alpha$-induced phosphorylation and nuclear translocation of the p65 subunit of NF-kB and AP-1 in all cell types studied. RES also inhibits the TNF- $\alpha-$ induced activation of mitogen-activated protein kinases (MAPKs) and abrogates TNFa-induced cytotoxicity and caspase activation. ${ }^{211}$ Similar results were reported by Kundu et al. in mouse skin stimulated with a prototype tumour promoter 12-Otetradecanoylphorbol-13-acetate. Their study revealed that topical application of RES inhibits activation of NF-KB, and phosphorylation of extracellular signal-regulated kinase (ERK) and p38 MAPK in 12-O-tetradecanoylphorbol-13-acetate-stimulated mouse skin. ${ }^{212,213}$

RES has an anti-inflammatory effect upon immune system cells. Sharma et al. found that RES suppresses the activity of $T$ and $B$ cells, decreasing the expression of CD28 and CD80; it also inhibits the secretion of IFN-y, IL-1, IL-4, IL-6 and TNF- $\alpha$ by macrophages, while IL-10 production is increased. ${ }^{214}$ In addition, RES also have effect on DCs. Svajger et al. demonstrated that human DCs treated with RES lose their ability to produce IL-12(p70), but increase IL-10 production after activation. In addition, DCs treated with RES are poor stimulators of allogeneic $T$ cells and decrease their ability to induce $\mathrm{CD}^{+} \mathrm{T}$ cell migration. ${ }^{215}$ Moreover, Buttari et al. found that RES can prevent activation of human monocyte-derived DCs by glucose-treated albumin, exerting an inhibitory effect on DC surface maturation marker and cytokine expression. ${ }^{216}$ 
RES has inhibitory effects on the expression of cell adhesion molecules and attenuates expression of ICAM-1 in bovine aortic endothelial cells stimulated with IL-6 and TNF- $\alpha .{ }^{217}$ RES also blocks the expression of adhesion molecules, ICAM-1 and VCAM-1, on lipopolysaccharide (LPS)-induced endothelial dysfunction in human microvascular endothelial cells, by inhibiting NF-kB activation. ${ }^{218}$

The anti-inflammatory effect of RES has also been studied in epithelial cells. Zaidi et al. found RES inhibits secretion of IL-8/CXCL8 and suppresses ROS production in human gastric epithelial cells infected by Helicobacter pylori (H. pylori). Moreover, $H$. pylori-initiated morphological changes are markedly blocked by RES. ${ }^{219}$ More recently, Houser et al. found that RES inhibits MCP-1/CCL2 (a chemokine that regulates migration and infiltration of monocytes/macrophages) in human airway cells stimulated by TNF- $\alpha^{220}$

Some studies have suggested the use of RES as potent anti-inflammatory compound in allergic diseases like asthma. Donnelly et al. demonstrated that RES inhibits GM-CSF, NF-kB and COX-2 expressions, and IL-8/CXCL8 release in human primary airway epithelial cells. ${ }^{221}$ Meeyoung et al. found that RES inhibits increases in T-helper-2-type cytokines (IL-4 and IL-5) in plasma and bronchoalveolar lavage fluid, and also effectively suppresses airway hyperresponsiveness, eosinophilia and mucus hypersecretion in an ovalbumin-induced allergic mouse model of asthma. The efficacy of RES is therefore found to be similar to that of dexamethasone. ${ }^{222}$ More recently, Okada et al. described that RES reduces serum IgE production, anaphylactic reaction, and IL-13 and IFN- $\alpha$ production from the mesenteric lymph nodes and spleens of mice sensitised by intragastric administration of ovalbumin plus cholera toxin, and treated with RES. ${ }^{223}$

\section{II.4.1.1.2. Resveratrol and ocular disorders}

RES has also shown anti-inflammatory and antioxidant properties when applied as a treatment for some ocular disorders. Kubota et al. demonstrated that RES can prevent ocular inflammation and oxidative stress in a mouse model of uveitis induced by LPS. ${ }^{224}$ They found that orally-administered RES inhibits retinal leukocyte adhesion and reduces protein levels of MCP-1/CCL2 and ICAM-1 (inflammation-related molecules involved in leukocyte recruitment, ${ }^{225}$ and adhesion ${ }^{226}$ ) in the retina and retinal pigment epithelium-choroid of mice. They also found that LPS augmented 8hydroxydeoxyguanosine (8-OHdG, a marker of oxidative stress in DNA) and RES was able to reduce $8-\mathrm{OHdG}$ generation at $3 \mathrm{~h}$ after inducing uveitis. 
Other studies have shown that RES also has an antioxidant effect on the lens. Doganay et al. studied the effect of RES on a sodium selenite-induced experimental cataract in rats. ${ }^{227}$ They found that selenite induces cataract formation in rats and increases malondialdehyde (MDA) concentration, a marker of lipid peroxidation, following low level of reduced glutathione ( $\mathrm{GSH}$, a biological antioxidant). RES administered intraperitoneally decreases MDA levels and cataract formation, and increases GSH in lens of treated rats, compared to untreated rats. Li et al. reported similar results in an in-vitro experiment using porcine and human primary lens epithelial cells, under both chronic hyperoxic oxygen condition and acute oxidative stress. ${ }^{228}$ They demonstrated that RES increases human lens epithelial cell survival and Forkhead box-O expression (a family of transcription factors involved in DNA repair and apoptosis). Zheng et al. described similar results in a human lens epithelial cell line exposed to $\mathrm{H}_{2} \mathrm{O}_{2}{ }^{229}$ They found that RES is able to reduce intracellular ROS production and p38 and c-Jun N-terminal kinase (JNK) phosphorylation, and to increase the expression levels of antioxidant enzymes such as superoxide dismutase (SOD)-1 and catalase. The anti-inflammatory effect of RES has also been demonstrated in primary porcine trabecular meshwork cells. Chronic treatment of trabecular meshwork cells with RES exposed to a chronic oxidative stimulus $\left(40 \% \mathrm{O}_{2}\right)$ prevents the increase of intracellular ROS, and IL-1 $\beta$, IL-6 and IL-8/CXCL8 cytokine secretion, along with antiapoptotic effects. ${ }^{230}$

RES exerts its anti-inflammatory effects not only on cytokine production and cells-mediated inflammation, but also on bacterial-induced inflammation of the ocular surface. Marino et al. exposed ex-vivo rabbit corneas to Staphylococcus aureus (S. aureus). ${ }^{231}$ They found $S$. aureus increases the expression of TLR -2 and upregulates IL-8/CXCL8 gene expression on corneal epithelial cells. However, RES decreases cell surface TLR-2 and downregulate IL-8/CXCL8 gene expression in cells exposed to $S$. aureus and treated with RES.

The eye, particularly the cornea, are continuously exposed to UV radiation which can provoke inflammation and oxidative stress. ${ }^{232-234}$ Due to its chemical structure, RES has also been shown to have a protective effect on the eye under UV-B exposure. Chou et al. showed that RES inhibits protein kinase $B$ (or AKT) and p38 MAPK phosphorylation, when retinal pigment epithelial cells are irradiated with UV$B{ }^{235}$ In addition, Kubota et al. found that RES protects mouse retina from white light exposure by reducing the number of TUNEL-positive photoreceptor cells and AP-1, the major transcription factor that regulates cellular cycle, differentiation and apoptosis. ${ }^{236}$ 


\section{II.4.2. Flavonoids}

The name flavonoid refers to the Latin word "flavus" meaning yellow, and includes more than 6500 natural compounds. These molecules are secondary metabolites of plants involved in defence against pathogens or UV radiation. Flavonoids are found in several natural sources such as onions, broccoli and leeks, among others. Although they are also part of the human diet, they are not considered as nutrients. Flavonoids have shown several biological effects including antibacterial, ${ }^{237}$ antiviral, ${ }^{238}$ and anticancer ${ }^{239}$ properties. Yet, similarly to stilbenes, there is greater interest in their anti-inflammatory properties. ${ }^{240}$

The basic flavonoid structure is the flavan nucleus, which consists of 15 carbon atoms arranged in three rings (C6-C3-C6). The various classes of flavonoids differ in the level of oxidation and pattern of substitution of the rings (see Figure 9). Therefore, flavonoids can be subdivided into six groups on the basis of their molecular structure: anthocyanins, flavanols, flavanones, flavones, flavonols and isoflavones. ${ }^{182}$ Flavonols are the most ubiquitous flavonoids in food, and one of the most important flavonols is quercetin (QCT).

\section{II.4.2.1. Quercetin}

QCT (3,3',4',5,7-pentahydroxyflavone, molecular weight $=302.24 \mathrm{~g} / \mathrm{mol}$; CAS 117-39-5; Figure 12) was first discovered, along with other flavonoids, by Nobel Prize laureate Albert Szent-Gyorgyi in the 1930s. It is chemically characterised by a phenylbenzo( $\mathrm{Y}$ )pyrone-derived structure consisting of two benzene rings linked through a heterocyclic pyran ring. The three rings are planar with hydroxyl groups attached. The molecule is quite polarised. QCT is a yellow powder entirely insoluble in cold water, poorly soluble in hot water, but quite soluble in lipids, alcohol $(2 \mathrm{mg} / \mathrm{mL})$ and DMSO (30 $\mathrm{mg} / \mathrm{mL}$ ). In addition, QCT is a lipophilic molecule and has high membrane permeability. Therefore, according to the biopharmaceutical classification system, it could also be considered as a class-II compound, like RES. ${ }^{194}$ 
<smiles>O=c1c(O)c(-c2ccc(O)c(O)c2)oc2cc(O)cc(O)c12</smiles>

Figure 12. Chemical structure of quercetin

QCT is the most abundant flavonol in food, representing $75 \%$ of the total intake. ${ }^{241}$ It is found in a variety of foods including apples, berries, onions and tea, as well as many seeds. QCT is also found in some medical botanical plants and trees, such as Ginkgo biloba. The amount of QCT intake in a normal diet is $5-40 \mathrm{mg} / \mathrm{day}$, but this can reach $200-500 \mathrm{mg} /$ day when consumption of fruits and vegetables containing QCT is high. ${ }^{242}$ QCT is metabolised in various organs such as the small intestine, colon liver and kidney. Concentrations of QCT in plasma are in the nanomolar range and depend on dosage intake. ${ }^{243} \mathrm{QCT}$ is generally well tolerated; doses up to $1 \mathrm{~g} /$ day for several months do not produce side-effects in humans. ${ }^{244}$

QCT, like RES, has both anti-inflammatory and antioxidant properties that have also led to an exponential growth of the scientific literature regarding its biological properties (Figure 13).

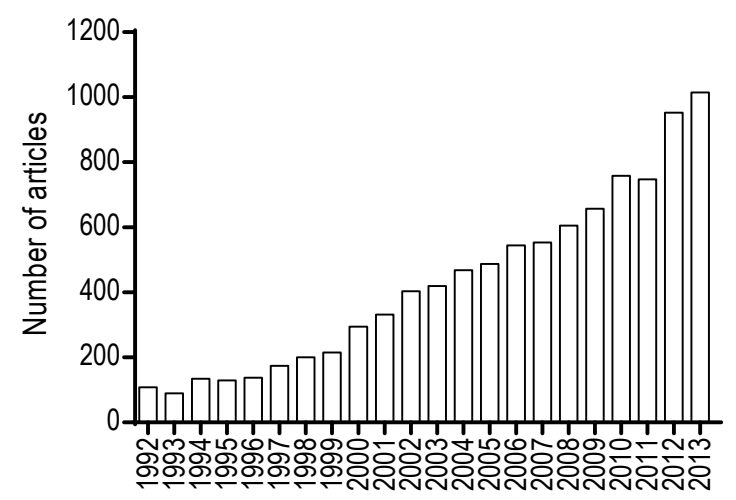

Figure 13. The number of articles in PubMed published every year using "quercetin" as keyword.

\section{II.4.2.1.1. Biological properties}

QCT, like other flavonoids, has anticancer, antiviral, cardioprotective and neuroprotective effects. ${ }^{245,246}$ Among these effects, QCT is also well-known for its antioxidant properties, being one of the most effective free radical scavengers. ${ }^{247}$ In- 
vivo studies support the antioxidant effect of QCT. For example, Kamaraj et al. showed that QCT supplementation ( $25 \mathrm{mg} / \mathrm{kg}$ body weight) in Swiss albino mice decreases lipid peroxidation and tumour marker enzymes (aryl hydrocarbon hydroxylase, 5'nucleotidase and lactate dehydrogenase) and increases antioxidant enzymes (SOD, catalase, glutathione peroxidase and glutathione reductase) in benzo(a)pyrene-induced carcinogenesis of mouse lung. ${ }^{248}$ The antioxidant effect of QCT has been shown, not only in in-vitro and in-vivo animal research, but also in humans. A daily dose of QCT for 6 weeks was shown to decrease plasma concentration of artherogenic oxidised low density lipoprotein in overweight and obese subjects with metabolic syndromes. ${ }^{249,250}$ In addition, QCT decreases serum MDA (an indicator of lipid peroxidation) levels in long distance runners, after 6 weeks of supplementation. ${ }^{251}$

Nevertheless, despite its numerous biological effects, this thesis will focus on the anti-inflammatory properties of QCT.

\section{II.4.2.1.1.1. Anti-inflammatory properties}

QCT has several anti-inflammatory effects, targeting multiple intracellular signalling pathways such as signal transducer and activator of transcription 1 (STAT1), NF-KB activation and MAPK family phosphorylation. ${ }^{252,253}$ For example, Comalada et al. reported the in-vitro and in-vivo effects of QCT in bone marrow-derived macrophages and in an experimental model of rat colitis induced by dextran sulfate sodium, respectively. They found, both in-vitro and in-vivo, that QCT is able to down-regulate the inflammatory response of inhibiting cytokines (TNF- $\alpha$ and IL-1 $\beta$ ) and inducible nitric oxide synthase (iNOS, an enzyme that generates the high reactive nitric oxide-NO-) expression through inhibition of the NF-KB pathway, without modification of JNK activity. ${ }^{254}$

Several in-vitro studies using different cell lines and animal models have shown that QCT can inhibit LPS-induced cytokine production. For instance, in-vitro data have shown that QCT inhibits LPS-induced TNF- $\alpha$ and IL-6 secretion in macrophages, ${ }^{25,256}$ and LPS-induced IL-8/CXCL8 production in lung cells. ${ }^{257}$ Moreover, it was shown that QCT can inhibit LPS-induced mRNA levels of TNF- $\alpha$ and IL-1 $\alpha$ in glial cells. ${ }^{258}$ Similar results were described by Qureshi et al. in peritoneal macrophages from mice stimulated with LPS. These results demonstrated that QCT inhibits LPS-induced expression of several pro-inflammatory genes (IL-1 $\alpha$, IL-1 $\beta$, IL-6, TNF- $\alpha$, IL-12, VCAM1, ICAM-1 and COX-2). ${ }^{259}$ Moreover, in macrophages QCT strongly reduces activation of phosphorylated ERK and p38 MAPK, and inhibits NF-KB activation through stabilisation of the NF-KB//KB complex and IKB degradation. ${ }^{260,261}$ Similar results were 
reported in Chang liver cells where QCT produces a significant decrease of iNOS and COX-2, as well as inhibition of mRNA level of iNOS and COX-2. ${ }^{262}$ In addition, QCT inhibits NF-KB activation and protein concentration of the phosphorylated form of the inhibitor $\mathrm{IKBa}{ }^{262}$

QCT also exerts its anti-inflammatory effect upon epithelial cells. For example, Nanua et al. demonstrated that QCT reduces TNF- $\alpha$-induced IL-8/CXCL8 and MCP1/CCL2 expression in cultured human airway epithelial cells. ${ }^{263}$ QCT also inhibits TNFa-induced phosphatidylinositol 3-kinase activity and AKT phosphorylation (enzymes involved in cellular functions, such as cell growth and proliferation), intracellular $\mathrm{H}_{2} \mathrm{O}_{2}$ production, NF-KB activation and IL-8/CXCL8 promoter activity. ${ }^{263}$ Similar antiinflammatory effects were shown in alveolar basal epithelial cells, where QCT inhibits IL-8/CXCL8 and GM-CSF release, as well as NF-KB and AP-1 and CAMP response element binding protein-dependent transcription to a greater extent than dexamethasone. ${ }^{221}$ These results have suggested that QCT has the ability to inhibit TNF- $\alpha$ transcription by inhibiting phosphorylation and activation of JNK. Therefore, QCT can suppress activation of the transcription factor AP-1, ERK1/2 and p38 MAPK activities, which are important in the post-transcriptional regulation of TNF- $\alpha$ mRNA. ${ }^{264}$ In-vivo experiments also support the anti-inflammatory properties of QCT. Muthigan et al. showed that QCT ameliorates experimental allergic encephalomyelitis in mice (a Th1 cell-mediated autoimmune disease) by blocking IL-12 signalling and Th1 differentiation. ${ }^{265}$ QCT also ameliorates autoimmune myocarditis induced in rats by decreasing TNF- $\alpha$ and IL-17, and by increasing IL-10 cytokine secretions, both in serum and culture supernatants from lymph node cells. ${ }^{266}$

Similar combined anti-inflammatory and anti-allergy properties of QCT have been found in animal models of allergic airway disease. Joskova et al. found that QCT causes bronchodilation in guinea pigs stimulated by ovalbumin after 21 days, reducing hyper-reactivity of airways. ${ }^{267}$ In the same in-vivo model, Jung et al. described that QCT reduces recruitment of leukocytes, particularly neutrophils and eosinophils, during the late-phase response of allergic response. ${ }^{268}$ Indeed, QCT inhalation has the same effect as oral administration, showing similar anti-asthmatic activity to cromolyn sodium and dexamethasone. ${ }^{269}$ In a murine model of allergic airway inflammation, Rogerio et al. showed that QCT inhibits NF-KB activation and reduces both IL-4 and IL-5 levels in plasma, ${ }^{270}$ and eosinophil and neutrophil counts/infiltration in lung tissue. ${ }^{271}$ In addition, using the same in-vivo model, Park et al. found that QCT regulates Th1/Th2 balance, inhibiting asthmatic reactions. ${ }^{272}$ Moreover, QCT suppresses the lgE response by reducing plasma histamine in Wistar rats experimentally sensitised to have an anaphylactic reaction to peanut. ${ }^{273}$ 


\section{II.4.2.1.2. Quercetin and ocular disorders}

The antioxidant and anti-inflammatory properties of QCT have been investigated, not only in cell lines from major organs, but also in some types of ocular tissues and cell lines. One of the first biomedical effects described for QCT in the eye, more that 30 years ago, was its inhibitory action on lens aldose reductase, which can provoke lens opacity. ${ }^{274}$ Oxidative stress is an initiating factor in the development of maturity-onset cataract and Cornish et al. showed that QCT exerts its effect on lens transparency following oxidative stress induced by $\mathrm{H}_{2} \mathrm{O}_{2} \cdot{ }^{275}$ Moreover, Cao et al. demonstrated that QCT can protect human lens epithelial cells from DMSO-induced apoptosis, by upregulating Bax expression. ${ }^{276}$

QCT was also investigated for its effects on suppression of retinal $S$ antigeninduced intraocular inflammation in Lewis rats by Romero et al. ${ }^{277}$ QCT administered intraperitoneally reduces uveal and retinal inflammation, vasculitis and perivasculitis. Morphometric analysis revealed that QCT also reduces choroidal thickness, when compared with control animals. These results clearly show the anti-inflammatory effect of QCT in experimental uveitis.

QCT, like RES, has a protective effect on the eye under UV exposure. Kaidzu et al. studied the antiapoptotic and antioxidant effects of QCT on light-exposed rat retina. They found that QCT alleviates histological damage, as shown by decreasing numbers of TUNEL positive cells and reduced expression of oxidative markers such as 8-OHdG, in rat retina exposed to light. ${ }^{278}$ Focusing on the lens, UV radiation is related to cataract formation. Jiang et al. studied the effect of QCT in human lens epithelial cells exposed to both UV radiation and $\mathrm{H}_{2} \mathrm{O}_{2} \cdot{ }^{279}$ They found that both $\mathrm{UV}$ and $\mathrm{H}_{2} \mathrm{O}_{2}$ induce a decrease in collagen type I and activates JNK and its downstream component, c-Jun, in both a time- and dose-dependent manner. By contrast, QCT protects against UV- and $\mathrm{H}_{2} \mathrm{O}_{2}$ induced decrease of collagen type I and inhibits both UV- and $\mathrm{H}_{2} \mathrm{O}_{2}$-induced JNK and c-Jun activation. 

III. Hypothesis 

The development of this thesis study was dependent upon the following hypothesis:

The topical application of quercetin, resveratrol and their combination has an anti-inflammatory effect on ocular surface diseases, such as dry eye and ocular allergy, which is due to modulation of epithelial cells and immune cell response. 

IV. Objectives 

In order to corroborate the hypothesis, a general objective and further specific objectives were established:

\section{General objective:}

To study the potential effect of quercetin, resveratrol and their combination on cells involved in the inflammatory process of immune-based ocular surface diseases such as dry eye disease and ocular allergy.

\section{Specific objectives:}

- To determine the anti-inflammatory effect of quercetin, resveratrol and their combination on conjunctival and corneal cell lines stimulated by TNF- $\alpha$.

- To determine the antioxidant effect of quercetin, resveratrol and their combination on conjunctival and corneal cell lines irradiated by UV-B light.

- To determine the immunomodulatory activity of quercetin, resveratrol and their combination in peripheral blood mononuclear cells.

- To determine the therapeutic effect of topical quercetin, resveratrol and their combination in a murine model of dry eye disease.

- To determine the effect of quercetin, resveratrol and their combination on mast cell degranulation. 



\section{Material and methods}



This section describes the methodology followed in this thesis and it has been divided into four sections, according to the established objectives in section IV. Firstly, the effect of QCT, RES and their combination was studied on ocular surface epithelial cells stimulated with either TNF- $\alpha$ or UV-B light. Secondly, the immunoregulatory effect of polyphenols was determined in peripheral blood mononuclear cells. After that, the anti-inflammatory effect of QCT, RES and their combination was confirmed in an invivo model of DED. And finally, the anti-allergic effect of QCT, RES and their combination was studied in cord blood-derived mast cells.

\section{VI.1. In-vitro model of inflammation and oxidative stress in human conjunctival and corneal epithelial cells}

The anti-inflammatory and antioxidant effects of QCT, RES and their combination (QCT+RES) were tested in-vitro using two established ocular surface cell lines, one derived from human conjunctival epithelium (IOBA-NHC) and other derived from human corneal epithelium (HCE), under two different stimuli: 1) TNF- $\alpha$-induced inflammation ${ }^{154}$ and 2) UV light-induced oxidative stress. ${ }^{280}$

The cytotoxicity of polyphenols on epithelial cells was firstly assessed, in order to determine the non-toxic concentrations for further experiments. Following this, the effect of QCT, RES and their combination on cytokine/chemokine secretion, COX-2 expression and ROS production were assessed.

\section{VI.1.1. Reagents}

All reagents and antibodies (Table 3) used in this part of the thesis have been grouped based on the manufacturer:

- Invitrogen (Inchinnan, UK): Dulbecco's Modified Eagle Medium/Nutrient Mixture F12 (DMEM/F12), alamarBlue ${ }^{\circledR} \quad$ cell viability assay, 4-(2-hydroxyethyl)-1piperazineethanesulfonic acid (HEPES).

- Nunc (Roskilde, Denmark): Plastic culture dishes and flasks.

- Panreac (Barcelona, Spain): EtOH and D-glucose.

- PeproTech EC (London, UK): Cytokine TNF-a.

- Sigma-Aldrich (St Louis, MO, USA): RES, QCT, DMEM (culture medium without sodium bicarbonate $\left(\mathrm{NaHCO}_{3}\right)$, sodium pyruvate and phenol red), 2',7'- 
dichlorodihydrofluorescein diacetate $\left(\mathrm{H}_{2} \mathrm{DCF}-\mathrm{DA}\right)$, L-glutamine, foetal bovine serum (FBS), cholera toxin, human epithelial growth factor (EGF), bovine insulin, penicillin, streptomycin, fungizone and hydrocortisone, DMSO, phosphate buffered saline (PBS), tris-hydrochloride (Tris- $\mathrm{HCl})$, sodium chloride $(\mathrm{NaCl})$, deoxycholic acid, Triton $X-100$, sodium dodecyl sulphate (SDS), ethylenediaminetetraacetic acid (EDTA), phenylmethanesulfonyl fluoride (PMSF), aprotinin, sodium orthovanadate $\left(\mathrm{Na}_{3} \mathrm{VO}_{4}\right)$, glycerol, 2-mercaptoethanol (2-ME), bromophenol blue, bovine serum albumin (BSA), Tween 20, tris-buffered saline (TBS).

- Thermo Fisher Scientific (Rockford, IL, USA): Bicinchoninic acid (BCA) assay.

\begin{tabular}{|c|c|c|c|}
\hline Antibody & Dilution & Manufacturer & Reference \\
\hline $\begin{array}{c}\text { Rabbit anti-COX-2 } \\
\text { Mouse anti-glyceraldehyde- } \\
\text { 3-phosphate } \\
\text { dehydrogenase (GAPDH) }\end{array}$ & $1 / 100$ & $\begin{array}{c}\text { Novus Biologicals } \\
\text { (Littleton, CO, USA) }\end{array}$ & NB100-689 \\
\hline $\begin{array}{c}\text { HRP-conjugated human } \\
\text { anti-IgG }\end{array}$ & $1 / 500$ & $\begin{array}{c}\text { Santa Cruz Biotechnology } \\
\text { (Heidelberg, Germany) }\end{array}$ & SC-166545 \\
\cline { 1 - 3 } $\begin{array}{c}\text { HRP-conjugated mouse } \\
\text { anti-lgG }\end{array}$ & $1 / 5000$ & $\begin{array}{c}\text { Jackson Laboratory (Bar } \\
\text { Harbor, ME, USA) }\end{array}$ & $715-035-150$ \\
\hline
\end{tabular}

Table 3. Antibodies used in this part of the thesis experiments (section V.1.8).

\section{VI.1.2. Preparation of polyphenol solutions}

QCT and RES were dissolved in EtOH. Fresh stock solutions of QCT and RES were prepared for each experiment, after which serial dilutions were carried out to achieve final concentrations, ranging from 0.5-25 $\mu \mathrm{M}$ QCT and 0.5-300 $\mu \mathrm{M}$ RES. Concentrations tested for each assay are detailed in Table 4.

Combinations of QCT and RES were prepared by mixing QCT and RES solutions, in order to reach the final concentrations for each experiment (as indicated in Table 4).

All solutions were prepared in such a way that the final concentration of vehicle, $0.5 \% \mathrm{EtOH}$, was non-toxic and was the same in all samples when polyphenols were added into each well. All solutions were kept away from light because light degrades polyphenols.

In order to clarify the rationale for the selected concentrations of polyphenols, the cytotoxicity of both compounds was firstly analysed for a range of concentrations 
(0.5-25 $\mu \mathrm{M}$ QCT and 0.5-300 $\mu \mathrm{M}$ RES). Once non-toxic concentrations of each compound for both cell lines were determined, non-toxic concentrations (0.5-25 $\mu \mathrm{M}$ QCT and 0.5-50 $\mu \mathrm{M}$ RES) were selected for dose-response studies in the two in-vitro models (TNF- $\alpha$-induced inflammation and UV-B-induced oxidative stress). Finally, one concentration of each compound (0.5 $\mu \mathrm{M}$ QCT and $5 \mu \mathrm{M}$ RES), which did not significantly decrease cytokine/chemokine secretion and ROS production in the doseresponse studies, was selected in order to analyse the effect of both compounds in combination. Regarding COX-2 assay, as the dose-response curve was not obtained, the highest concentrations of each compound tested on dose-response curves $(25 \mu \mathrm{M}$ QCT and $50 \mu \mathrm{M}$ RES) and the combination of both compounds tested previously (0.5 $\mu \mathrm{M} Q \mathrm{QCT}$ and $5 \mu \mathrm{M}$ RES) were used in this experiment in order to maintain the same concentrations tested. 


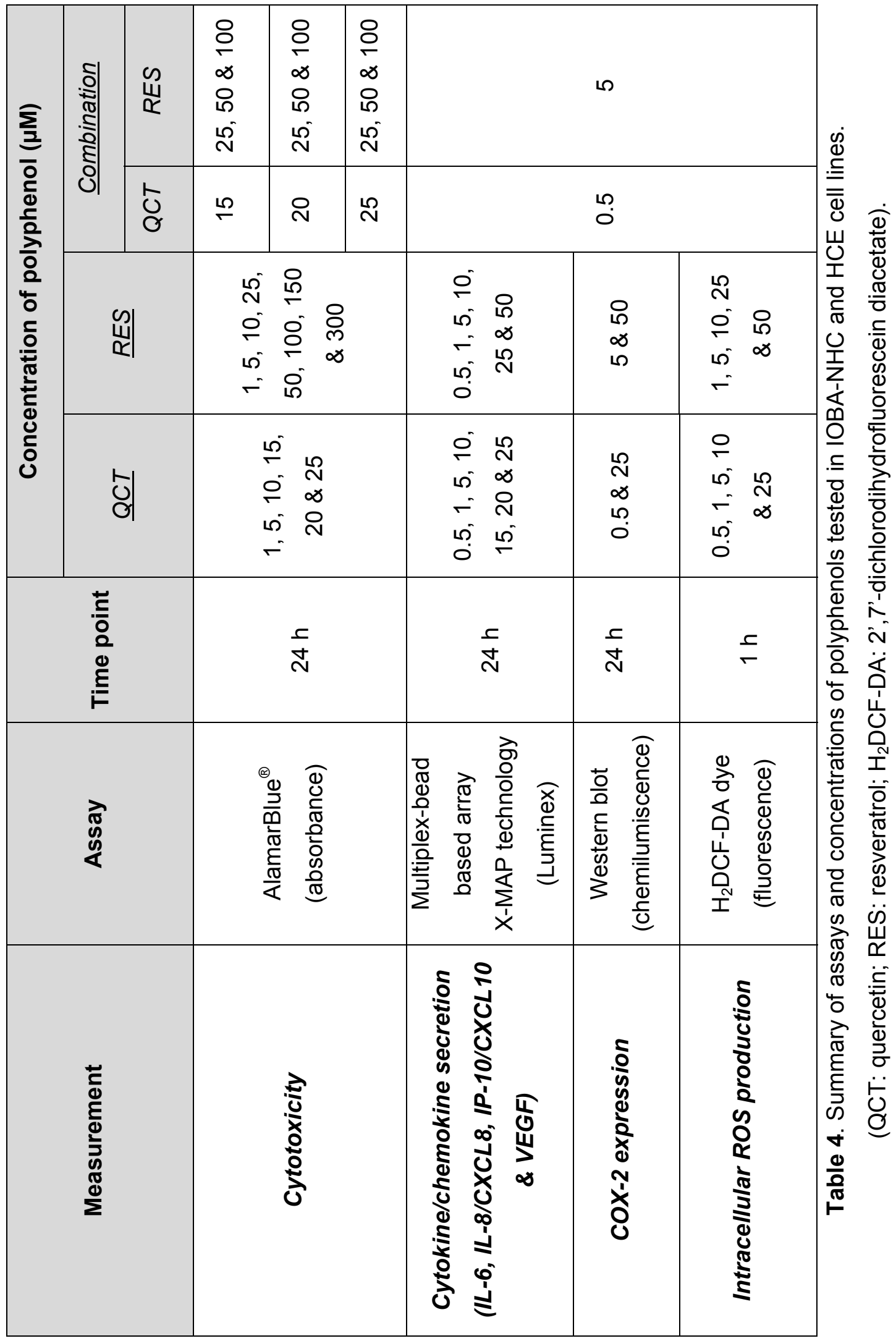




\section{VI.1.3. Cell lines and culture conditions}

Two different ocular surface epithelial cell lines, IOBA-NHC and HCE, were used for these experiments.

A) The IOBA-NHC is a non-transfected, spontaneously immortalised epithelial cell line derived from normal human conjunctiva. ${ }^{281}$ It was used from passage 62. IOBA-NHC cells were cultured in DMEM/F-12 L-glutamine supplemented with $10 \%$ FBS, $0.1 \mu \mathrm{g} / \mathrm{mL}$ cholera toxin, $2 \mathrm{ng} / \mathrm{mL}$ EGF, $1 \mu \mathrm{g} / \mathrm{mL}$ bovine insulin, $5000 \mathrm{U} / \mathrm{mL}$ penicillin, $5 \mathrm{mg} / \mathrm{mL}$ streptomycin, $2.5 \mu \mathrm{g} / \mathrm{mL}$ fungizone and $0.5 \mu \mathrm{g} / \mathrm{mL}$ hydrocortisone.

B) The HCE is an SV40-immortalised human corneal epithelial cell line ${ }^{282}$ kindly gifted by Professor Arto Urti (University of Helsinki, Finland). It was used from passage 45. HCE cells were cultured in DMEM/F-12 L-glutamine supplemented with $15 \%$ FBS, $0.5 \%$ DMSO, $0.1 \mu \mathrm{g} / \mathrm{mL}$ cholera toxin, $10 \mathrm{ng} / \mathrm{mL}$ EGF, $5 \mu \mathrm{g} / \mathrm{mL}$ insulin, $100 \mathrm{U} / \mathrm{mL}$ penicillin and $0.1 \mathrm{mg} / \mathrm{mL}$ streptomycin.

Both cell lines were cultured in an incubator at $37{ }^{\circ} \mathrm{C}$ in a humidified atmosphere of $5 \% \mathrm{CO}_{2}$. Media were changed every other day and cells were observed every day by phase contrast microscopy.

All experiments were carried out in DMEM culture medium without $\mathrm{NaHCO}_{3}$, sodium pyruvate and phenol red, and supplemented with $3.15 \mathrm{~g} / \mathrm{L}$ D-glucose, $2 \mathrm{mM} \mathrm{L-}$ glutamine and $25 \mathrm{mM}$ HEPES, hereinafter referred to as complete medium (CM).

\section{VI.1.4. Cytotoxicity assay}

The toxicity of QCT, RES and QCT+RES on epithelial cells was assessed by the resazurin reduction test. AlamarBlue ${ }^{\circledR}$, or resazurin, is a non-toxic and nonfluorescent compound that is reduced continuously by cells to resorufin, a fluorescence compound. This conversion occurs intracellularly by mitochondrial, microsomal and cytosolic oxidoreductases. ${ }^{283}$ AlamarBlue $^{\circledR}$ can distinguish metabolically active cells because non-viable cells have lower innate metabolic activity and hence they generate a proportionally lower signal than healthy cells. The fluorescence intensity of alamarBlue ${ }^{\circledR}$ reagent is directly proportional to cell number.

IOBA-NHC and HCE cells were seeded in 96-well plates at density of $10^{4}$ cells per well and grown for 3 days. After that, culture medium was replaced with serumfree, non-supplemented medium and cells were maintained in it for $24 \mathrm{~h}$. Then, the serum-free medium was replaced with $\mathrm{CM}$ and cells were treated with different 
concentrations of QCT, RES or QCT+RES (see Table 4 for details of polyphenol concentrations) and incubated for $24 \mathrm{~h}$ at $37^{\circ} \mathrm{C}$. Control cells were treated with vehicle $(0.5 \%$ EtOH). Following incubation, supernatants were discarded and $10 \%$ alamarBlue $^{\circledR}$, prepared in supplemented DMEM/F12 cultured medium, was added. Cells were incubated for $4 \mathrm{~h}$ at $37^{\circ} \mathrm{C}$. Finally, medium from each sample was collected and fluorescence was measured at $560 \mathrm{~nm}_{\mathrm{ex}} / 590 \mathrm{~nm}_{\mathrm{em}}$ by UV/Vis spectrophotometry (SpectraMax ${ }^{\circledR}$ M5, Molecular Devices, Sunnyvale, CA, USA). Three independent experiments were performed and measurements were from 8 replicates for each condition studied.

\section{VI.1.5. Cell cytokine stimulation and polyphenol treatments}

IOBA-NHC and HCE cells were seeded in 24-well plates at densities of $45 \times 10^{3}$ and $60 \times 10^{3}$ cells per well $\left(23 \times 10^{3}\right.$ and $32 \times 10^{3}$ cell/ $\left./ \mathrm{cm}^{2}\right)$ respectively, and grown for 3 days. For the COX-2 assay, cells were seeded and grown in $25 \mathrm{~cm}^{2}$ flasks at density of $60 \times 10^{3}$ cells per flasks until confluence. Culture medium was then replaced with serumfree, non-supplemented medium and cells were maintained in this for $24 \mathrm{~h}$ at $37^{\circ} \mathrm{C}$. Subsequently, serum-free medium was discarded and cells were pre-treated with QCT, RES, QCT+RES (see Table 4 for concentration details) or vehicle, in CM for $2 \mathrm{~h}$ at 37 ${ }^{\circ} \mathrm{C}$. After that, pre-treatments were removed, cells were stimulated with $25 \mathrm{ng} / \mathrm{mL}$ TNF$\alpha$ in the presence of QCT, RES, QCT+RES or vehicle, and incubated for $24 \mathrm{~h}$ in CM. Unstimulated cells treated with polyphenols but without TNF- $\alpha$ were used as control. After the treatment period, the conditioned media were collected and centrifuged at 59 $\mathrm{x} \mathrm{g}$ for $5 \mathrm{~min}$. Supernatants, and plates and flasks with adherent cells were stored at $80{ }^{\circ} \mathrm{C}$ until use. Three independent experiments were performed in duplicate, unless otherwise stated.

\section{VI.1.6. Cellular lysis and total protein assay}

Total protein content was measured in plates/flasks with adherent cells. Firstly, cells were lysed. The adherent cells were washed with cold PBS and disrupted with ice-cold radioimmunoprecipitation assay (RIPA) buffer [10 mM tris- $\mathrm{HCl}(\mathrm{pH} 7.4), 150$ $\mathrm{mM} \mathrm{NaCl}, 1 \%$ deoxycholic acid, $1 \%$ Triton X-100, $0.1 \%$ SDS, and $1 \mathrm{mM}$ EDTA] supplemented with two proteases inhibitors $(0.1 \mathrm{mg} / \mathrm{mL}$ PMSF and $60 \mu \mathrm{g} / \mathrm{mL}$ aprotinin), and a tyrosine phosphatase inhibitor $\left(0.1 \mu \mathrm{M} \mathrm{Na}_{3} \mathrm{VO}_{4}\right)$. Samples were incubated on ice for $30 \mathrm{~min}$. Then, samples were centrifuged at $18,000 \times \mathrm{g}$ for $30 \mathrm{~min}$ at $4{ }^{\circ} \mathrm{C}$. Finally, supernatants were collected and stored at $-80^{\circ} \mathrm{C}$ until use. 
Total protein was assessed using the BCA assay. This method combines the Biuret reaction (reduction of $\mathrm{Cu}^{+2}$ to $\mathrm{Cu}^{+1}$ by protein in an alkaline medium) with the highly sensitive colourimetric detection of $\mathrm{Cu}^{+1}$ using BCA. ${ }^{284}$ The purple-coloured reaction product of this assay is formed by the chelation of two molecules of BCA with one $\mathrm{Cu}^{+1}$. This complex exhibits a strong absorbance that is nearly linear with increasing protein concentrations. The BCA assay was carried out on cell lysates according to the manufacturer's instructions. Briefly, $25 \mu \mathrm{L}$ of standards and cell lysate samples were treated with $B C A$ working reagent $(B C A$ in $0.1 \mathrm{mM}$ sodium hydroxide and $4 \%$ cupric sulphate solution) and incubated for $30 \mathrm{~min}$ at $37^{\circ} \mathrm{C}$. Then, samples were cooled at room temperature and the absorbance was read at $562 \mathrm{~nm}$ using the SpectraMax ${ }^{\circledR}$ M5 UV/Vis spectrophotometer (Molecular Devices). Data were analysed using the software SoftMax ${ }^{\circledR}$ Pro (Molecular Devices).

\section{VI.1.7. Measurement of cytokine/chemokine secretion}

Cytokine/chemokine secretion was assessed by a multiplex-bead based array, using Luminex ${ }^{\mathrm{TM}} \mathrm{x}-\mathrm{MAP}^{\circledR}$ multiplexing bead technology. ${ }^{285,286}$ This assay consists of a series of microspheres (beads) containing fluorochromes of differing intensity embedded within the bead, giving each group of beads with a specific molecules attached a unique signal. The multiplex system enables the detection and quantification of multiple analytes (proteins and peptides, or nucleic acids) in a single sample volume.

IL-6, IL-8/CXCL8, IP-10/CXCL10 and VEGF levels were determined in cell supernatants with a commercial Milliplex 4-plex human cytokine/chemokine immunobead-based assay (HCYTO, Millipore, Watford, UK), according to the manufacturer's instructions. Briefly, $25 \mu \mathrm{L}$ of cell supernatant from each sample were incubated in 96-well plates with antibody-immobilised beads overnight at $4{ }^{\circ} \mathrm{C}$. Then, beads were washed and incubated with biotinylated cytokine/chemokine antibody solution for $1 \mathrm{~h}$ at room temperature, followed by incubation with streptavidinphycoerythrin for $30 \mathrm{~min}$ at room temperature. Finally, beads were washed and read on a Luminex ${ }^{\mathrm{TM}}$ 100-IS instrument (Luminex Corporation, Austin, Tx, USA). Standard curves of known concentrations of recombinant human cytokines/chemokines were used to convert fluorescent units to cytokine/chemokine concentration units $(\mathrm{pg} / \mathrm{mL})$. The minimum detectable level for each cytokine/chemokine, based on the manufacturer specifications, was: $0.3 \mathrm{pg} / \mathrm{mL}$ for IL-6; $0.2 \mathrm{pg} / \mathrm{mL}$ for IL-8/CXCL8; 1.2 $\mathrm{pg} / \mathrm{mL}$ for IP-10/CXCL10; and $5.8 \mathrm{pg} / \mathrm{mL}$ for VEGF. When a cytokine level was not 
detectable, the minimum detectable level was used in the analysis. Data were analysed with the BeadView ${ }^{\mathrm{TM}}$ Software (Upstate, UK) and normalised to total protein content for each sample.

\section{VI.1.8. Cyclo-oxygenase-2 expression}

The expression levels of COX-2 in cells were assessed by SDS-polyacrylamide gel electrophoresis (PAGE) and Western blotting, according to the Laemmli method. ${ }^{287}$ Cell homogenates from flasks were mixed with an equal volume of $2 X$ Laemmli $4 \%$ SDS, $20 \%$ glycerol, $10 \%$ 2-ME, $0.004 \%$ bromphenol blue and $0.125 \mathrm{mM}$ Tris- $\mathrm{HCl}$, $\mathrm{pH} 6.8]$.

Proteins in samples were denatured and reduced by boiling them for $5 \mathrm{~min}$ at $110^{\circ} \mathrm{C}$. Then, $20 \mu \mathrm{g}$ of total protein from each sample were separated by SDS-PAGE on $10 \%$ acrylamide gels. After SDS-PAGE, proteins in the gel were transferred to 0.2 $\mu \mathrm{m}$ pore size nitrocellulose membranes, blocked with $5 \%$ non-fat milk, $3 \%$ BSA and $0.05 \%$ Tween 20 in TBS for $1 \mathrm{~h}$ under agitation, at room temperature. Membranes were then incubated with rabbit anti-COX-2 antibody (1/100) overnight under agitation at $4{ }^{\circ} \mathrm{C}$. Subsequently, membranes were washed and incubated with HRP-conjugated human anti-lgG antibody (1/2000) diluted in blocking buffer for $1 \mathrm{~h}$ at room temperature. Membranes were washed and immunoreactive bands of COX-2 protein were detected by incubation of membranes in enhanced chemiluminiscent solution (Santa Cruz) for $1 \mathrm{~min}$. Images were captured by a charge-coupled device (CCD) camera in a ChemiDoc XRS (Bio-Rad, Inc., Hercules, CA, USA). GAPDH band was used as loading control to normalise the levels of COX-2 protein detected. Briefly, membranes were washed and incubated with Restore ${ }^{\mathrm{TM}}$ western blot stripping buffer (Thermo Fisher Scientific) for $1 \mathrm{~h}$ at $60^{\circ} \mathrm{C}$. Subsequently, membranes were washed, blocked and further incubated with primary mouse anti-GAPDH (1/500) antibody and secondary HRP-conjugated mouse anti-lgG antibody (1/5000), as described above. Quantification of protein immunoreactive bands was assessed by volumetric densitometry using Quantity One ${ }^{\circledR}$ software (Bio-Rad). Two experiments were performed in duplicate. Band intensity data were expressed as percentage of band intensity. 


\section{VI.1.9. Measurement of reactive oxygen species induced by UV-B radiation}

The generation of intracellular ROS by UV-B exposure of epithelial cells was assessed using $\mathrm{H}_{2}$ DCF-DA, which is a non-fluorescent dye that passively diffuses into cells, where it is cleaved and deacetylated to $\mathrm{H}_{2} \mathrm{DCF}$ by intracellular esterases. Nonfluorescent $\mathrm{H}_{2} \mathrm{DCF}$ is rapidly oxidised to fluorescent DCF by intracellular ROS.

IOBA-NHC and HCE cells were cultured in supplemented medium in 24 well UV transparent plates for $72 \mathrm{~h}$. Then, culture medium was discarded, serum-free nonsupplemented medium was added in which cells were maintained for $24 \mathrm{~h}$. After that, culture medium was replaced and cells were pre-treated with QCT, RES, QCT+RES (see table 4 for doses used) or vehicle for $1 \mathrm{~h}$ at $37^{\circ} \mathrm{C}$ in $\mathrm{CM}$. At that point, pretreatments were discarded, and cells were loaded with $\mathrm{H}_{2}$ DCF-DA adding $500 \mu \mathrm{L}$ of 10 $\mu \mathrm{M} \mathrm{H}_{2}$ DCF-DA solution in CM and incubated for $30 \mathrm{~min}$. $\mathrm{H}_{2}$ DCF-DA medium was then aspirated; cells were treated with QCT, RES, QCT+RES or vehicle at the same concentrations used before, and exposed to 8W UV-B lamps (with an excitation peak of $302 \mathrm{~nm}$ ), located $3 \mathrm{~cm}$ below cells. At that distance the UV-B radiation power density was $7.15 \mathrm{~mW} / \mathrm{cm}^{2}$, according to the manufacturer (Bio-Rad). Cells were irradiated for $15 \mathrm{~s}$ from the bottom of the well plate to avoid UV-B absorption by polyphenols in the culture media. After $15 \mathrm{~s}$, UV-B radiant exposure was $107.25 \mathrm{~mJ} / \mathrm{cm}^{2}$, as calculated with the following formula: $\mathrm{H}=\mathrm{E} \cdot \mathrm{t}$, where $\mathrm{H}$ is the radiant exposure $\left(\mathrm{J} / \mathrm{cm}^{2}\right), E$ is the irradiance $\left(\mathrm{W} / \mathrm{cm}^{2}\right)$ and $\mathrm{t}$ is the exposure time (s). Control cells were not irradiated. After UV-B exposure, cells were cultured for $1 \mathrm{~h}$ and then intracellular fluorescence was measured at $488 \mathrm{~nm}_{\mathrm{ex}}$ / $522 \mathrm{~nm}_{\mathrm{em}}$ using the SpectraMax ${ }^{\circledR}$ M5 UV/Vis spectrophotometer (Molecular Devices). Fluorescence data from each sample were normalised to the corresponding total protein content, determined previously in adherent cells by the BCA protein assay kit. Three different experiments were carried out and samples were performed in duplicate. 


\section{VI.2. In-vitro proliferative response of human T cells}

The immunosuppressive effect of QCT, RES and their combination QCT+RES was tested on human peripheral blood mononuclear cells (PBMCs). Cytotoxicity, and cell proliferation and viability were studied.

These experiments were carried out at University College London, under the supervision of Dr. Virginia Calder. The doctoral candidate's stay was supported by "ayudas para estancias breves en el desarrollo de tesis doctorales" by University of Valladolid in 2012. Additionally, some experiments were carried out at IOBA, University of Valladolid.

\section{VI.2.1. Reagents}

All reagents were purchased from Sigma-Aldrich, unless otherwise specified. All assays were performed in Roswell Park Memorial Institute (RPMI)-1640 HEPES Modification medium supplemented with $2 \mathrm{mM}$ L-glutamine, $10 \%$ FBS, $50 \mathrm{U} / \mathrm{mL}$ penicillin, $50 \mu \mathrm{g} / \mathrm{mL}$ streptomycin, non-essential amino acids (glycine, L-alanine, Lasparagine, L-aspartic acid, L-glutamic acid, L-proline and L-serine), $1 \mathrm{mM}$ sodium pyruvate and $2.5 \mu \mathrm{M}$ 2-ME. Propidium iodide $(\mathrm{PI})$ was also obtained from Sigma.

Anti-CD3 (clone HIT3a) and anti-CD28 (clone 28.2) were purchased from Becton Dickinson Biosciences (BD, Oxford, UK). Carboxyfluorescein diacetate succinimidyl ester (CFSE) and Ficoll-Hypaque were purchased from Invitrogen. Foetal calf serum (FCS) was purchased from Labtech International (Ringmer, UK).

\section{VI.2.2. Preparation of polyphenol solutions}

QCT, RES and QCT+RES solutions were prepared as previously described (see Section V.1.2), with some modifications.

Stock solutions of QCT and RES were prepared in EtOH, after which serial dilutions were carried out to achieve final concentrations ranging from 0.5-25 $\mu \mathrm{M} Q C T$ and $0.5-100 \mu \mathrm{M}$ RES. Aliquots of each solution were kept at $-20^{\circ} \mathrm{C}$. Concentrations tested for each assay are detailed in Table 5 . The final concentration of vehicle in cells treated with QCT or RES alone was a non-toxic concentration of $0.5 \% \mathrm{EtOH}$ in the culture medium.

Final concentrations tested of QCT+RES were achieved by adding QCT solutions and RES solutions to each sample in order to reach the final concentrations 
in cultured medium as indicated in Table 5. The final concentration of vehicle in cells treated with QCT+RES was a non-toxic concentration of $1 \% \mathrm{EtOH}$ in the culture medium.

In order to clarify the rationale for the selected concentrations of polyphenols, the cytotoxicity of both compounds was firstly analysed for a range of concentrations (0.5-25 $\mu \mathrm{M} \mathrm{QCT} \mathrm{and} \mathrm{0.5-100} \mathrm{\mu M} \mathrm{RES,} \mathrm{alone} \mathrm{and} \mathrm{in} \mathrm{combination).} \mathrm{After} \mathrm{that,} \mathrm{low}$ concentrations of QCT $(0.5,1,5$ and $10 \mu \mathrm{M})$ and RES $(5$ and $10 \mu \mathrm{M})$ were selected in order to study their effect on PBMC proliferation. These latter concentrations were selected based on the previous experiment, although some of them were toxic for PBMCs. This issue is discussed in Section VII.2 because both experiments were not comparable. 


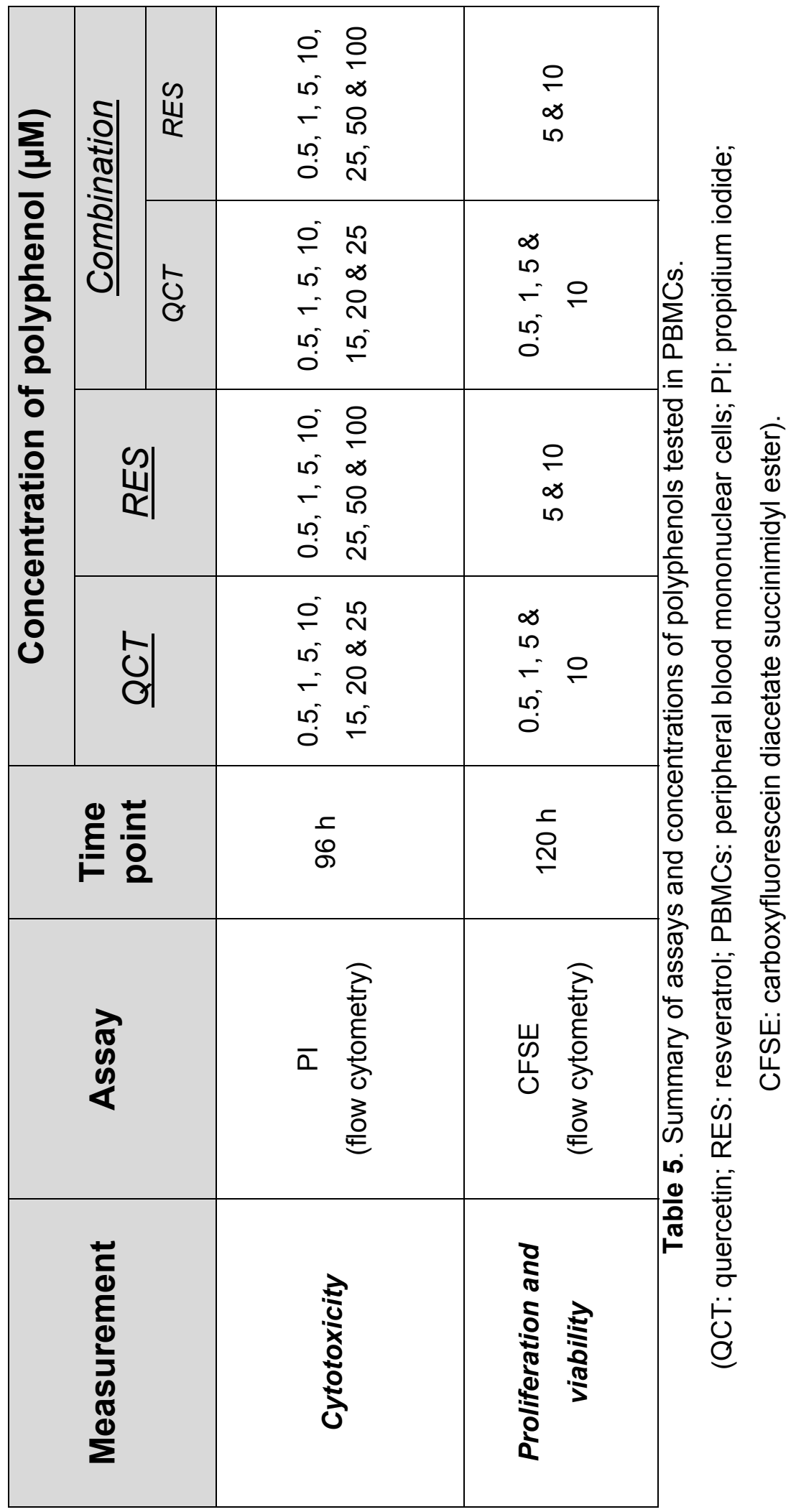




\section{VI.2.3. Donors}

Peripheral blood was obtained from 3 anonymous healthy donors with informed consent. Exclusion criteria included a history of autoimmune, inflammatory or allergic disease, haematological disorder or current usage of systemic medication. The protocols used in this study were reviewed and approved by the Local Ethics Committee ("UCL committee" and "Comité Ético de la Universidad de Valladolid", the latter included in appendix III). All studies involving human subjects were conducted according to the tenets of the Declaration of Helsinki.

\section{VI.2.4. Peripheral blood mononuclear cell isolation}

PBMCs were isolated by Ficoll-Hypaque density gradient centrifugation. Peripheral blood collected using heparinised tubes was diluted with an equal volume of culture medium. Diluted blood was slowly layered over a Ficoll-Hypaque solution and centrifuged at $624 \times \mathrm{g}$ for $40 \mathrm{~min}$ at room temperature, with no brake. Mononuclear cells were then collected, washed by adding excess of culture medium and centrifuged at $193 \times \mathrm{g}$ for $10 \mathrm{~min}$ at room temperature. After washing, PBMCs were resuspended in RPMI culture medium for further experiments.

\section{VI.2.5. Cytotoxicity assay}

The cytotoxicity of polyphenols alone and in combination on PBMCs was assayed by $\mathrm{PI}$ staining by means of flow cytometry. $\mathrm{PI}$ is a membrane impermeant fluorescence dye that binds to DNA and RNA. Late stages of apoptosis are characterised by loss of membrane permeability and DNA fragmentation and, consequently, loss of nuclear DNA content. Hence, PI penetrates into cells undergoing apoptosis and binds to fragmented DNA, identifying dead cells.

PBMCs were seeded in 96-well plates at density of $2 \times 10^{5}$ cells per well and treated with different concentrations of QCT, RES, QCT+RES (see Table 5 for concentration details) or vehicle. After that, PBMCs were stimulated with $50 \mathrm{ng} / \mathrm{mL}$ antiCD3 and $10 \mu \mathrm{g} / \mathrm{mL}$ anti-CD28, and incubated in $5 \% \mathrm{CO}_{2}$ for $96 \mathrm{~h}$ at $37{ }^{\circ} \mathrm{C}$. Control cells were stimulated in presence/absence of vehicle. At the end of incubation, $5 \mu \mathrm{g} / \mathrm{mL}$ $\mathrm{PI}$ were added and cells were acquired using a FACSCalibur ${ }^{\mathrm{TM}}$ flow cytometer (BD). At least 15,000 events were analysed using CellQuest ${ }^{\mathrm{TM}}$ Pro software (BD). Samples were previously gated on forward scatter (FS) versus side scatter (SS) to exclude debris and clumps. All cells were further gated to assess level of viable cells (PI- 
negative). Three different experiments were carried out and samples were performed in single samples.

\section{VI.2.6. Proliferation and viability assay}

The effect of polyphenols on PBMC proliferation and viability was assessed by means of the CFSE dye. ${ }^{288}$ CFSE is a colourless and non-fluorescent dye that passively diffuses into cells. CFSE becomes highly fluorescent when acetate groups are cleaved by intracellular esterases. When a cell divides the daughter cells each comprise half of the parental cell. Hence CFSE passes equally on to the daughter cells upon cell division and it is able to trace the division of cells by the dilution of CFSE dye during the first and subsequent cell divisions.

Cells were incubated with $5 \mu \mathrm{M}$ CFSE in serum-free non-supplemented medium for $10 \mathrm{~min}$ at $37{ }^{\circ} \mathrm{C}$ in a water bath. After that, $1 \mathrm{~mL}$ of cold stop buffer (10\% FCS in RPMI) was added to cells and were then incubated for $30 \mathrm{~min}$ at room temperature, washed once with RPMI, and further resuspended to reach a concentration of $2 \times 10^{5}$ cells per well. PBMCs were then treated with different concentrations of QCT, RES, QCT+RES (see Table 5 for more details) or vehicle, stimulated with $50 \mathrm{ng} / \mathrm{mL}$ anti-CD3 and $10 \mu \mathrm{g} / \mathrm{mL}$ anti-CD28, and incubated in $5 \% \mathrm{CO}_{2}$ for $120 \mathrm{~h}$ at $37^{\circ} \mathrm{C}$. At the end of incubation period, cells were counterstained with $\mathrm{PI}(5 \mu \mathrm{g} / \mathrm{mL})$. Samples were acquired using FACSCalibur flow cytometer, and listmode data were generated using CellQuest Pro software. Data were further analysed using WinList (Verity Software House, Topsham, ME, USA). Samples were gated on FS versus SS to exclude debris and clumps. For proliferation, cells were gated in a CFSE/PI dot plot to track the divisions of CFSE-labelled cells enabling identification of the percentage of divided (proliferated) cells. Percentage of divided cells was obtained by the formula:

$$
\text { Cell division }(\%)=(\text { Divided cells } / \text { Divided cells }+ \text { Undivided cell }) \times 100
$$

where "divided cells" were PI-negative/CFSE-negative, and "undivided cells" were PInegative/CFSE-positive.

For the viability assay, all lymphocytes were gated to assess the levels of PInegative cells (live cells). Gates for CFSE-positive and PI-negative cells were set on viable lymphocytes at day 0.

In all cases, treatments were performed in triplicate for each experiment and three different experiments were carried out. 


\section{VI.3. Experimental murine model of dry eye}

The anti-inflammatory effect of QCT, RES and their combination (QCT+RES) was tested in an experimental murine model of DED, followed by an adoptive transfer model. ${ }^{87,94}$ Corneal fluorescein staining (CFS), aqueous tear production (TP), goblet cell count, cytokine levels in tears and $\mathrm{CD}^{+} \mathrm{T}$ cell infiltration in conjunctiva were assessed (Figure 14).



Figure 14. Timeline for experimental DED and adoptive transfer model. (TP: tear production; CK: cytokine/chemokine production; CFS: corneal fluorescein staining; GC: goblet cell count).

These experiments were carried out in Allergan headquarters (Irvine, CA, USA) under the supervision of Dr. Michael E. Stern, Vice-President of the Inflammation Research Program. The doctoral candidate's stay was supported by "ayudas para estancias breves en el desarrollo de tesis doctorales" by University of Valladolid in 2011 and 2013.

\section{VI.3.1. Animals}

Female C57BL/6 and female T cell deficient nude C57BL/6 mice, both 8-10 weeks old, were purchased from Taconic Farms (Oxnard, CA, USA). Animal studies approval was obtained from the Institutional Animal Care and Use Committee at Allergan. All studies adhered to the Association for Research in Vision and Ophthalmology (ARVO) statement for the use of animals in ophthalmic and vision research.

\section{VI.3.2. Desiccating stress-induced dry eye and topical treatments}

Experimental DED was induced in female C57BL/6 mice (hereinafter referred to as donor mice) as previously described. ${ }^{87,94}$ Up to five mice were placed in a cage with a perforated plastic screens on two sides of the cage. Mice were exposed to DS by 
placing them in a controlled-environment chamber with airflow from fans (two fans on each side of the cage) for $10 \mathrm{~h}$ a day, and room relative humidity at $20 \%$ and temperature maintained at $23{ }^{\circ} \mathrm{C}$. A continuous dose of $0.1 \mathrm{mg} /$ day scopolamine hydrobromide (Sigma-Aldrich) was administered by subcutaneously implanted osmotic pump (Alzet ${ }^{\circledR}$, Cupertino, CA, USA). DS was carried out for 10 days. Control mice were kept in a non-stressed environment between $50 \%$ and $80 \%$ relative humidity and temperature of $21-23^{\circ} \mathrm{C}$, without exposure to forced airflow.

Mice were randomly divided into 6 groups, each group with 9 mice:

1) Control group (no DS and no topical treatment).

2) DS (no topical treatment).

3) DS + vehicle (vehicle was confidentially developed by Allergan).

4) DS + $0.01 \%$ QCT.

5) $\mathrm{DS}+0.1 \%$ RES.

6) $\mathrm{DS}+0.01 \% \mathrm{QCT}+0.1 \%$ RES.

The treatments started one day before DS was set up and they were administered topically in both eyes $(5 \mu \mathrm{L} / \mathrm{eye})$, three times a day. At the end of the experiment, mice were euthanised with $\mathrm{CO}_{2}$ and left eyeballs (with attached lids) were immediately embedded in optimal cutting temperature compound (OCT; VWR, Suwanee, GA, USA) and flash frozen. Right eyeballs with attached lids were also excised and kept in $4 \%$ paraformaldehyde at $4{ }^{\circ} \mathrm{C}$, until use.

\section{VI.3.2.1. Corneal staining in donor mice}

CFS was used to evaluate corneal epithelial damage caused by DS in donor mice after 9 days. A dose of $5 \mu \mathrm{L}$ of $0.125 \%$ fluorescein sodium salt (Sigma) was applied into the lateral conjunctival sac of the mouse and $1 \mathrm{~min}$ later both corneas were examined, using a stereo microscope under cobalt blue light. Superficial punctate staining was recorded in a masked fashion using the National Eye Institute grading system, scoring 0 to 3 (Grade $0=$ no punctate staining to less than 5 . Grade $1=5-20$ punctates; Grade 2 = 20-50 punctates; Grade $3>50$ punctates) for each of 5 areas of both corneas: central, superior, inferior, nasal and temporal. ${ }^{289}$

\section{VI.3.2.2. Aqueous tear production in donor mice}

TP was performed the day before DS induction and after 9 days of DS in donor mice, using phenol red-impregnated cotton threads (Zone-Quick; Lacrimedics, Eastsound, WA, USA). The thread was held with sterilised jeweller forceps and placed 
in the lateral cantus of the right eye for $30 \mathrm{~s}$. Threads turn from yellow to red on absorption of tears. Wetting of the thread was measured using the millimeter scale on the cotton thread package.

\section{VI.3.2.3. Tear collection in donor mice}

Tear samples were collected on day 6 and 10 in donor mice. A volume of $1.5 \mu \mathrm{L}$ of cytokine assay buffer (Millipore) was instilled into each eye, and immediately $2 \mu \mathrm{L}$ (1 $\mu \mathrm{L} / \mathrm{eye}$ ) of tear fluid and buffer were collected from the tear meniscus in the lateral cantus with a glass capillarity tube and a microcap (Drummond Scientific, Broomall, PA, USA). Then, the tear sample pooled from both eyes was diluted in $8 \mu \mathrm{L}$ of Beadlyte assay buffer and stored at $-80^{\circ} \mathrm{C}$ until the time of assay.

\section{VI.3.2.4. Measurement of cytokine and chemokine levels in donor mouse tears}

The level of selected cytokines and chemokines in the tears was evaluated using a multiplex bead analysis with $\mathrm{x}-\mathrm{MAP}^{\circledR}$ technology (as previously described in Section V.1.7). Cytokine/chemokine tear levels were assessed using a Milliplex 15-plex

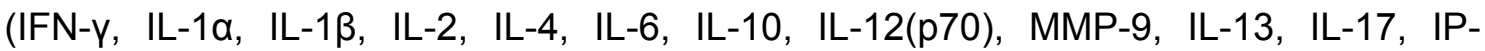
10/CXCL10, MCP-1/CCL2, RANTES/CCL5 and TNF- $\alpha$ ) mouse cytokine/chemokine immunobead-based assay (Millipore) and analysed on a Luminex 200 instrument (Luminex Corporation). The minimum detectable level for each cytokine/chemokine, based on the manufacturer specifications, was: $1.1 \mathrm{pg} / \mathrm{mL}$ for IFN- $\gamma, 10.3 \mathrm{pg} / \mathrm{mL}$ for IL1a, $5.4 \mathrm{pg} / \mathrm{mL}$ for IL-1ß, $1.0 \mathrm{pg} / \mathrm{mL}$ for IL-2, $0.4 \mathrm{pg} / \mathrm{mL}$ for IL-4, $1.1 \mathrm{pg} / \mathrm{mL}$ for IL-6, 2.0 $\mathrm{pg} / \mathrm{mL}$ for IL-10, $4.8 \mathrm{pg} / \mathrm{mL}$ for IL-12(p70), $0.38 \mathrm{pg} / \mathrm{mL}$ for MMP-9, $8.3 \mathrm{pg} / \mathrm{mL}$ for IL-13, $0.5 \mathrm{pg} / \mathrm{mL}$ for IL-17, $0.8 \mathrm{pg} / \mathrm{mL}$ for IP-10/CXCL10, $6.7 \mathrm{pg} / \mathrm{mL}$ for MCP-1/CCL2, 2.7 $\mathrm{pg} / \mathrm{mL}$ for RANTES/CCL5 and $2.3 \mathrm{pg} / \mathrm{mL}$ for TNF- $\alpha$. When a cytokine level was not detectable, the minimum detectable level was used in the analysis. Data were analysed using Milliplex analyst software.

\section{VI.3.2.5. Goblet cell count in donor mice}

Right eyeballs with attached lids from donor mice fixed in $4 \%$ paraformaldehyde were further embedded in paraffin. Eight-micrometer sections were stained with periodic acid-Schiff (PAS) reagent. Sections were viewed by light microscopy using an Eclipse E400 (Nikon, Melville, NY, USA) with a 20x objective. For quantification of goblet cells, a midline section of each eye was counted following the morphometric guideline of the entire superior and inferior conjunctiva, starting at the 
limbus and spanning the entire length to the tarsal conjunctiva, including the conjunctival epithelium and stroma to a depth of $75 \mu \mathrm{M}$ below the basement membrane. Goblet cells were counted in a masked fashion.

\section{VI.3.2.6. Immunohistochemistry in donor mice}

$\mathrm{CD}^{+} \mathrm{T}$ cell immunostaining and counting were performed as previously described. ${ }^{39,94,290,291}$ OCT-embedded left eyeballs (with attached lids) from donor mice were sectioned at $7 \mu \mathrm{m}$ thickness with a cryostat microtome (CM3050 S; Leica Microsystems, Buffalo Grove, IL, USA), mounted on glass slides and stored at $-80^{\circ} \mathrm{C}$. On the day of use, the mounted sections were dried at $37{ }^{\circ} \mathrm{C}$ overnight and fixed in -20 ${ }^{\circ} \mathrm{C}$ acetone for $10 \mathrm{~min}$ at room temperature. Endogenous peroxidases were then quenched with $\mathrm{H}_{2} \mathrm{O}_{2}$ for 10 min, slides were rinsed with PBS and air dried for $1 \mathrm{~h}$. After that, non-specific sites were blocked with $20 \%$ normal rabbit serum (Dako, Carpinteria, CA; USA), and rat anti-mouse CD4 primary antibody (BD) was added to each slide and incubated for $1 \mathrm{~h}$ at room temperature. After slides were extensively washed, sections were incubated with biotinylated polyclonal anti-rat IgG secondary antibody (BD) for 30 min. Then, Vectastain Elite $A B C$ reagent (Vector laboratories, Burlingame, CA, USA) was added for antigen localisation, following manufacturer's instructions. Finally, samples were incubated with NovaRED (Vector Laboratories) peroxidase substrate to give a red stain, and subsequently counterstained with haematoxylin (Invitrogen). In addition, rat anti-mouse lgG isotype (BD) was used as negative control. CD4 ${ }^{+} \mathrm{T}$ cells in the conjunctiva were countered in a masked fashion by light microscopy, using an Eclipse E400 (Nikon). For quantification of $\mathrm{CD}^{+}{ }^{+} \mathrm{T}$ cells, a midline section of each eye was counted following the morphometric guideline of the entire superior and inferior conjunctiva, starting at the limbus and spanning the entire length to the tarsal conjunctiva, including the conjunctival epithelium and stroma to a depth of $75 \mu \mathrm{M}$ below the basement membrane. $\mathrm{CD} 4^{+} \mathrm{T}$ cells were counted in a masked fashion.

\section{VI.3.3. Adoptive transfer model}

$\mathrm{CD}^{+} \mathrm{T}$ cells from each group of donor mice (DS and control mice) were isolated from spleens and cervical lymph nodes, and enriched using the CD4 ${ }^{+} \mathrm{T}$ cell isolation kit II (MACS System, Miltenyi Biotec, Auburn, CA, USA), according to the manufacturer's instructions. Then, one donor-equivalent of cell suspension in PBS was transferred intraperitoneally into each T cell deficient nude C57BL/6 mouse (hereinafter referred to as recipient mouse), as previously described. ${ }^{39,94}$ One donor-equivalent is 
defined as the number of cells remaining after the respective in-vitro manipulation (i.e. $\mathrm{CD}^{+} \mathrm{T}$ cells) of a single set of lymph nodes or spleen (approximately $5 \times 10^{6} \mathrm{CD}^{+} \mathrm{T}$ cells). Eight recipient mice per donor group were used. Recipient mice were sacrificed $72 \mathrm{~h}$ after adoptively transferred $\mathrm{CD} 4^{+} \mathrm{T}$ cells. Immediately, left eyeballs with attached lids were embedded in OCT and flash frozen, and right eyeballs with attached lids were also excised and kept in $4 \%$ paraformaldehyde at $4{ }^{\circ} \mathrm{C}$ until use.

\section{VI.3.3.1. Aqueous tear production in recipient mice}

TP was performed in recipient mice one day before and $48 \mathrm{~h}$ after transferring $\mathrm{CD}^{+} \mathrm{T}$ cells. TP was measured following the same procedure described in Section V.3.2.2.

\section{VI.3.3.2. Tear collection in recipient mice}

Tear samples were collected on day 3 in recipient mice following the same procedure described in Section V.3.2.3.

\section{VI.3.3.3. Measurement of cytokine and chemokine levels in recipient mouse $\underline{\text { tears }}$}

The level of selected cytokines and chemokines (IFN- $-\mathrm{l}, \mathrm{IL}-1 \alpha, \mathrm{IL}-1 \beta, \mathrm{IL}-2, \mathrm{IL}-4$, IL-6, IL-10, IL-12(p70), MMP-9, IL-13, IL-17, IP-10/CXCL10, MCP-1/CCL2, RANTES/CCL5 and TNF- $\alpha$ ) in the tears of recipient mice was evaluated using a multiplex bead analysis with $\mathrm{x}-\mathrm{MAP}^{\circledR}$ technology, as previously described in Sections V.1.7 and V.3.2.4. The minimum detectable level for each cytokine/chemokine, based

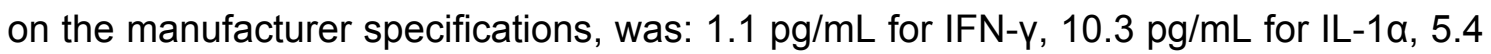
$\mathrm{pg} / \mathrm{mL}$ for IL-1 $\beta, 1.0 \mathrm{pg} / \mathrm{mL}$ for IL-2, $0.4 \mathrm{pg} / \mathrm{mL}$ for IL-4, $1.1 \mathrm{pg} / \mathrm{mL}$ for IL-6, $2.0 \mathrm{pg} / \mathrm{mL}$ for IL-10, $4.8 \mathrm{pg} / \mathrm{mL}$ for IL-12(p70), $0.38 \mathrm{pg} / \mathrm{mL}$ for MMP-9, $8.3 \mathrm{pg} / \mathrm{mL}$ for IL-13, $0.5 \mathrm{pg} / \mathrm{mL}$ for IL-17, $0.8 \mathrm{pg} / \mathrm{mL}$ for IP-10/CXCL10, $6.7 \mathrm{pg} / \mathrm{mL}$ for MCP-1/CCL2, $2.7 \mathrm{pg} / \mathrm{mL}$ for RANTES/CCL5 and $2.3 \mathrm{pg} / \mathrm{mL}$ for TNF- $\alpha$. When a cytokine level was not detectable, the minimum detectable level was used in the analysis. Data were analysed using Milliplex analyst software. 


\section{VI.3.3.4. Goblet cell count in recipient mice}

In order to count goblet cell numbers in the conjunctiva, right eyeballs from recipient mice (with attached lids) and previously fixed in $4 \%$ paraformaldehyde, were embedded in paraffin, sectioned and stained as described in Section V.3.2.5.

\section{VI.3.3.5. Immunohistochemistry in recipient mice}

OCT-embedded left eyeballs, with attached lids, from recipient mice were sectioned and analysed as described in Section V.3.2.6 for counting $\mathrm{CD}^{+} \mathrm{T}$ cell infiltration in conjunctiva. 


\section{VI.4. In-vitro model of conjunctival mast cell response}

The anti-allergic effect of QCT, RES and their combination (QCT+RES) was tested on human cord blood-derived mast cells (CBMCs). ${ }^{292}$ Cell viability, and histamine and cytokine secretions were assessed.

These experiments were carried out in at University College London, under the supervision of Dr. Virginia Calder. The doctoral candidate's stay was supported by "ayudas para estancias breves en el desarrollo de tesis doctorales" by University of Valladolid in 2012.

\section{VI.4.1. Reagents}

Stemspan $^{\mathrm{TM}}$ serum-free medium was purchased from StemCell Technologies (Grenoble, France).

Stem cell factor (SCF) and cytokines IL-3 and IL-6 were purchased from Peprotech. FCS was purchased from Labtech. Human IgE was obtained from Abcam (Cambridge, MA, USA). PI and anti-human lgE ( $\varepsilon$-chain specific) antibody was obtained from Sigma.

\section{VI.4.2. Preparation of polyphenol solutions}

QCT, RES and QCT+RES solutions were prepared as previously described in Section V.1.2.

Stock solutions of QCT and RES were prepared in EtOH, after which serial dilutions were carried out to achieve final concentrations ranging from 0.5-25 $\mu \mathrm{M} Q C T$ and 0.5-100 $\mu \mathrm{M}$ RES. Cells treated with each polyphenol alone had a final concentration of $0.5 \% \mathrm{EtOH}$ for dose-response curves. Aliquots of each solution were kept at $-20^{\circ} \mathrm{C}$. Concentrations tested for each assay are detailed in Table 6 .

Final concentration testing of QCT+RES for preliminary studies (two experiments performed in single samples) were achieved by adding QCT solutions and RES solutions to each sample in order to reach the final concentrations in cultured medium, as indicated in Table 6. The final concentration of vehicle was a non-toxic concentration of $1 \% \mathrm{EtOH}$ in the culture medium. 
A combined QCT and RES solution for a final experiment, performed in triplicate, was prepared by mixing a QCT solution and a RES solution in order to reach the final concentrations of $10 \mu \mathrm{M}$ QCT $+25 \mu \mathrm{M}$ RES, and $0.5 \%$ EtOH as vehicle.

In order to clarify the rationale for the selected concentrations of polyphenols, the cytotoxicity of both compounds was firstly analysed for a range of concentrations (0.5-25 $\mu \mathrm{M}$ QCT and 0.5-100 $\mu \mathrm{M}$ RES, alone and in combination). Once non-toxic concentrations were determined, dose-response curves to QCT (0.5-25 $\mu \mathrm{M})$ and RES $(0.5-100 \mu \mathrm{M})$ for histamine release were determined (the same concentrations of QCT and RES were also selected in order to study their effect on cytokine release in a further study). Finally, one concentration of each compound (10 $\mu \mathrm{M}$ QCT and $25 \mu \mathrm{M}$ RES), which did not significantly decrease histamine release in the dose-response study, was selected in order to analyse the effect of both compounds in combination on histamine release and cytokine secretion. 


\begin{tabular}{|c|c|c|c|c|c|c|c|}
\hline \multirow{4}{*}{ 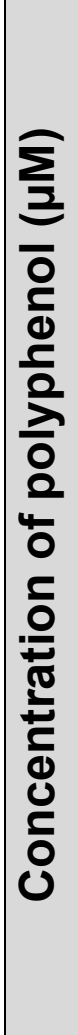 } & \multirow{2}{*}{ 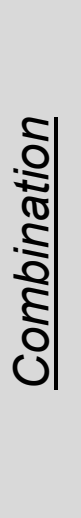 } & $\underset{w}{\mathbb{x}}$ & $\begin{array}{cc}\circ & \circ \\
\circ & \circ \\
10 & \infty \\
\sigma & 0 \\
5 & 0 \\
0 & 0 \\
0 & \sim\end{array}$ & 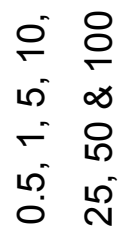 & \multicolumn{3}{|c|}{$\stackrel{\sim}{\sim}$} \\
\hline & & ơ &  & 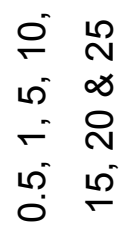 & \multicolumn{3}{|c|}{$\stackrel{\circ}{-}$} \\
\hline & \multicolumn{2}{|c|}{$\begin{array}{l}\omega \\
\frac{\omega}{\alpha}\end{array}$} & 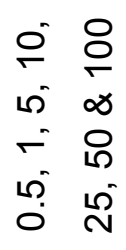 & 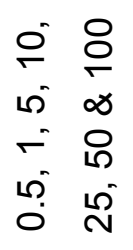 & & $\begin{array}{cc}0 & 8 \\
0 & 0 \\
10 & \infty \\
\sigma & 0 \\
5 & 10 \\
0 & \infty\end{array}$ & \\
\hline & \multicolumn{2}{|c|}{$\stackrel{-}{ত}$} & 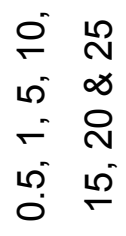 &  & & 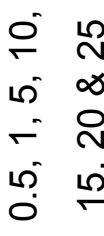 & \\
\hline & \multicolumn{2}{|l|}{ 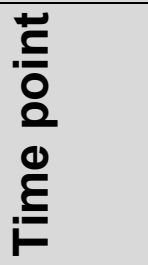 } & $\frac{\ulcorner}{\stackrel{\sim}{N}}$ & $\stackrel{ᄃ}{\leftarrow}$ & \multicolumn{3}{|c|}{$\stackrel{c}{\stackrel{\sim}{\sim}}$} \\
\hline & \multicolumn{2}{|l|}{ 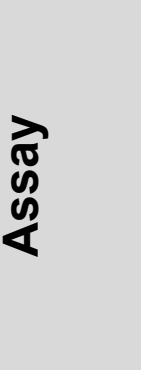 } & 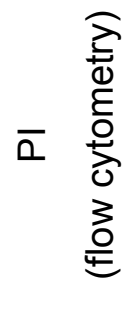 & 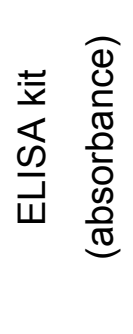 &  & 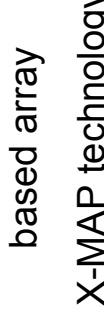 & 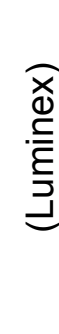 \\
\hline & \multicolumn{2}{|l|}{ 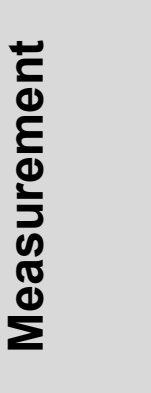 } & 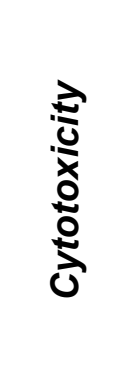 & 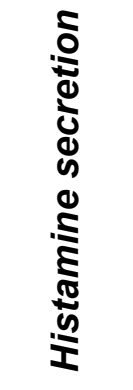 & 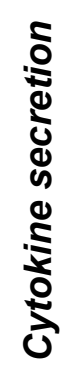 & 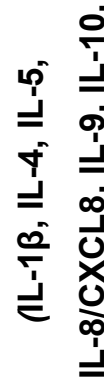 & 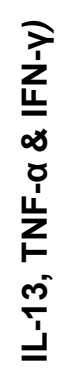 \\
\hline
\end{tabular}




\section{VI.4.3. Cord blood-derived mast cells}

Commercially available cord blood CD34 ${ }^{+}$stem cells (Lonza, Wokingham, UK) were cultured as previously described ${ }^{293}$ in Stemspan serum-free medium, supplemented with $100 \mathrm{ng} / \mathrm{mL}$ SCF and $50 \mathrm{ng} / \mathrm{mL}$ IL-6. Additionally, $1 \mathrm{ng} / \mathrm{mL}$ IL-3 was added to cultured medium during the first 14 days of culture. From week 8 to 11 , cell culture medium was further supplemented with $10 \%$ FCS. After 11 weeks of culture, cells were characterised [CD117 ${ }^{+}$(c-kit); > $90 \%$ tryptase-positive, > $90 \%$ chymasepositive] using a FACSCalibur flow cytometer (BD) and used for experiments between 11 and 16 weeks of culture.

\section{VI.4.4. Sensitisation and activation of mast cells}

CBMCs were activated by cross-linking surface $\lg E$ with an anti-lgE antibody, as previously described. ${ }^{292,293}$ CBMCs were seeded in 96-well plates at a density of $2 \times 10^{5}$ cells per well in $200 \mu \mathrm{L}$ of Stemspan medium supplemented with $100 \mathrm{ng} / \mathrm{mL}$ SCF. Cells were then pre-sensitised by adding human $\operatorname{lgE}(4 \mu \mathrm{g} / \mathrm{mL})$ and were incubated for $18 \mathrm{~h}$ at $37^{\circ} \mathrm{C}$. Cells not exposed to IgE were used as a control. After that, anti-human IgE antibody $(50 \mu \mathrm{g} / \mathrm{mL})$ was added to $\operatorname{lgE}$-sensitised cells and further incubated for $24 \mathrm{~h}$ at $37^{\circ} \mathrm{C}$.

\section{VI.4.5. Polyphenol treatments}

After $18 \mathrm{~h}$ of pre-sensitisation with IgE, QCT, RES, QCT+RES or vehicle were added to samples and cells were then incubated for $30 \mathrm{~min}$ prior to add anti-lgE. Cellfree supernatants were collected at $1 \mathrm{~h}$ and $24 \mathrm{~h}$ after anti-lgE activation and stored at $80^{\circ} \mathrm{C}$ for subsequent analysis (Table 6 ).

\section{VI.4.6. Cytotoxicity assay}

In order to determine the non-toxic concentration of polyphenols on CBMCs, cell toxicity after exposure to different concentrations of QCT, RES or QCT+RES (see Table 6 for more details) and vehicle were assessed $24 \mathrm{~h}$ after $\operatorname{lgE} /$ anti-lgE activation by PI staining using flow cytometry, as previously described in Section V.2.5. Briefly, 24 $\mathrm{h}$ after sensitisation/activation of CBMCs and polyphenol treatments, $5 \mu \mathrm{g} / \mathrm{mL} \mathrm{PI}$ was added to cells and immediately afterwards cells were acquired using a FACSCalibur flow cytometer (BD). At least 15,000 events were analysed using CellQuest Pro software (BD). Samples were previously gated on FS versus SS to exclude debris and 
clumps. All cells were further gated to assess level of viable cells (PI-negative). One experiment was carried out and samples were performed in triplicate for testing polyphenols individually and in single samples for testing polyphenol mixtures.

\section{VI.4.7. Histamine release}

The concentration of histamine was measured in cell-free supernatants after $1 \mathrm{~h}$ of IgE/anti-lgE activation, using a commercial enzyme-linked immunosorbent assay (ELISA) kit (IBL, Hamburg, Germany). The assay was conducted following the manufacturer's instructions. First, $50 \mu \mathrm{L}$ of supernatants and standards were acylated and diluted before being placed into plate. Then, peroxidase-conjugated histamine and histamine antiserum were added to each sample. The plate was incubated for $3 \mathrm{~h}$, washed and developed with tetramethylbenzidine solution. Finally, absorbance was measured at $450 \mathrm{~nm}$ using a microplate reader (Titertek ${ }^{\mathrm{TM}}$, Vienna, Austria). Doseresponse curves were obtained from two experiments performed in single samples. A further experiment were performed in triplicate for testing the effect of QCT and RES combination (10 $\mu \mathrm{M} \mathrm{QCT}+25 \mu \mathrm{M}$ RES) on histamine secretion.

\section{VI.4.8. Cytokine secretion}

IL-1 $\beta$, IL-4, IL-5, IL-8/CXCL8, IL-9, IL-10, IL-13, TNF- $\alpha$ and IFN- $\gamma$ levels were determined in cell supernatants after $24 \mathrm{~h}$ using Luminex bead technology with a commercial Milliplex 9-plex human cytokine/chemokine immunobead-based assay (HCYTOMAG, Millipore), as previously described in Section V.1.7. The minimum detectable levels for each cytokine/chemokine were $0.8 \mathrm{pg} / \mathrm{mL}$ for $\mathrm{IL}-1 \beta, 4.5 \mathrm{pg} / \mathrm{mL}$ for IL-4, $0.5 \mathrm{pg} / \mathrm{mL}$ for IL-5, $0.4 \mathrm{pg} / \mathrm{mL}$ for IL-8/CXCL8, $1.2 \mathrm{pg} / \mathrm{mL}$ for IL-9, $1.1 \mathrm{pg} / \mathrm{mL}$ for IL$10,1.3 \mathrm{pg} / \mathrm{mL}$ for IL-13, $0.7 \mathrm{pg} / \mathrm{mL}$ for TNF- $\alpha$ and $0.8 \mathrm{pg} / \mathrm{mL}$ for IFN-ץ. When a cytokine level was not detectable, the minimum detectable level was used in the analysis. Doseresponse curves were obtained from two experiments performed in single samples. A further experiment were performed in triplicate for testing the effect of QCT and RES combination (10 $\mu \mathrm{M}$ QCT + $25 \mu \mathrm{M}$ RES) on cytokine secretion. 


\section{VI.5. Statistical Analysis}

For in-vitro experiments, all data were expressed as mean \pm standard error of the mean (SEM). Statistics were analysed using the SPSS software package (SPSS version 15.0 for Windows, SPSS Inc., Chicago, IL, USA). Homogeneity of variances was analysed using Levene's test. Cytotoxicity, cytokine secretion, COX-2 expression and intracellular ROS production data from IOBA-NHC and HCE cells were analysed using the $t$-test or $t$-test with Welch correction for comparison of unstimulated cells versus stimulated cells, and one-way analysis of variance (ANOVA) with Dunnett's post-hoc test or Games-Howell test for intergroup comparisons. Cytotoxicity, and proliferation and viability data from PBMCs were analysed using the $t$-test or $t$-test with Welch correction. Cytotoxicity, histamine secretion and cytokine/chemokine production from CBMCs were analysed using the $t$-test or $t$-test with Welch correction.

For the in-vivo model of DED and adoptive transfer model, data were expressed as median \pm interquartile range (the difference between the 25 th and 75 th percentiles) using Tukey's method for plotting the whiskers and outliers. The Mann-Whitney test was used for statistical comparisons (outliers obtained from Tukey's method were included in the statistical analysis).

Two-sided p-values equal to or less than 0.05 were considered statistically significant. 


\section{Results}



Results have been divided into four sections following the same scheme of the chapter "material and methods" chapter:

1. In-vitro model of inflammation and oxidative stress in human conjunctival and corneal epithelial cells.

2. In-vitro proliferative response of human T cells.

3. Experimental murine model of DED.

4. In-vitro model of conjunctival mast cell response. 



\section{VI.1. In-vitro model of inflammation and oxidative stress in human conjunctival and corneal epithelial cells}

This section summarises all data obtained from testing the cytotoxicity, antiinflammatory and antioxidant effects of polyphenols on conjunctival and corneal epithelial cells.

\section{VI.1.1. Cytotoxicity of polyphenols}

The cytotoxicity of QCT, RES and QCT+RES in conjunctival and corneal epithelial cells after $24 \mathrm{~h}$ exposure was tested using the alamarBlue ${ }^{\circledR}$ assay.

Prior to testing polyphenols in both epithelial cell lines, it was verified that the vehicle used for polyphenol solubilisation $(0.5 \% \mathrm{EtOH})$ did not have any effect on conjunctival and corneal epithelial cells (as shown in Figure 15).
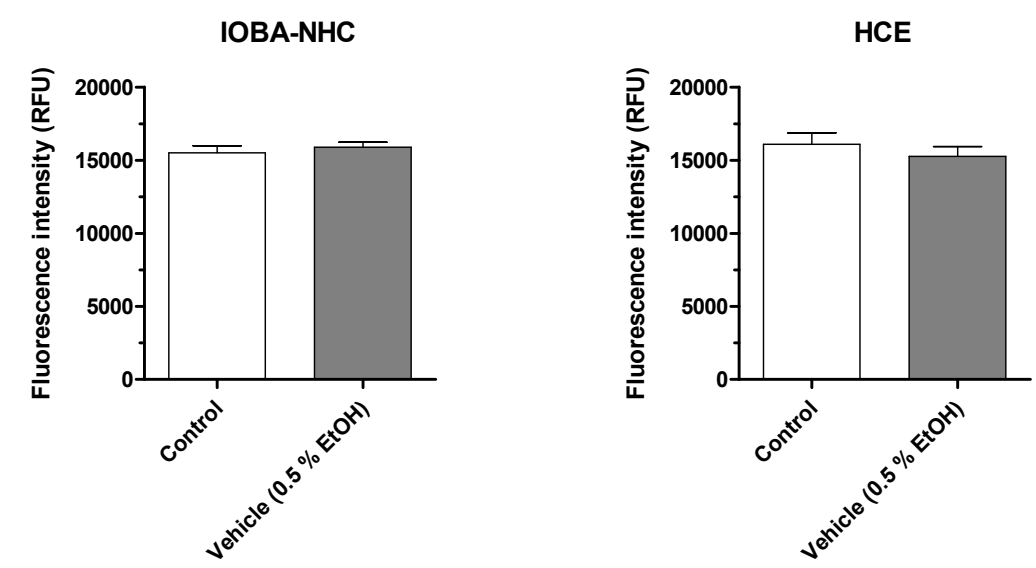

Figure 15. Effect of $0.5 \%$ ethanol (EtOH) on both IOBA-NHC and HCE cells. $\mathrm{N}=3$.

\section{VI.1.1.1. Cytotoxicity in IOBA-NHC cells}

Figure $16(A-C)$ shows the fluorescence intensity, in relative fluorescence units (RFU), for IOBA-NHC cells treated with QCT, RES and QCT+RES. QCT did not decrease IOBA-NHC cell viability at any concentration tested (1, $510,15,20$ and 25 $\mu \mathrm{M}$ ) compared to vehicle (Figure 16A). Moreover, a significant increase in fluorescence intensity was found at $20 \mu \mathrm{M} Q C T(p<0.05)$. Regarding RES concentrations tested (1, $5,10,25,50,100$ and $300 \mu \mathrm{M}$ ), only $300 \mu \mathrm{M}$ RES provoked a significant decrease of cell viability $(p<0.05)$, compared to vehicle (Figure 16B). IOBA-NHC cells were also treated with 15, 20 and $25 \mu \mathrm{M}$ QCT in combination with each 25, 50 and $100 \mu \mathrm{M}$ RES concentrations. There was a significant decrease in fluorescence intensity when HCE 
cells were treated with $25 \mu \mathrm{M}$ QCT $+25 \mu \mathrm{M}$ RES $(p<0.01), 20 \mu \mathrm{M}$ QCT $+50 \mu \mathrm{M}$ RES (p<0.05), $15 \mu \mathrm{M}$ QCT + $100 \mu \mathrm{M}$ RES $(p<0.05)$ and $20 \mu \mathrm{M}$ QCT + $100 \mu \mathrm{M}$ RES $(p<0.05$; Figure 16C).
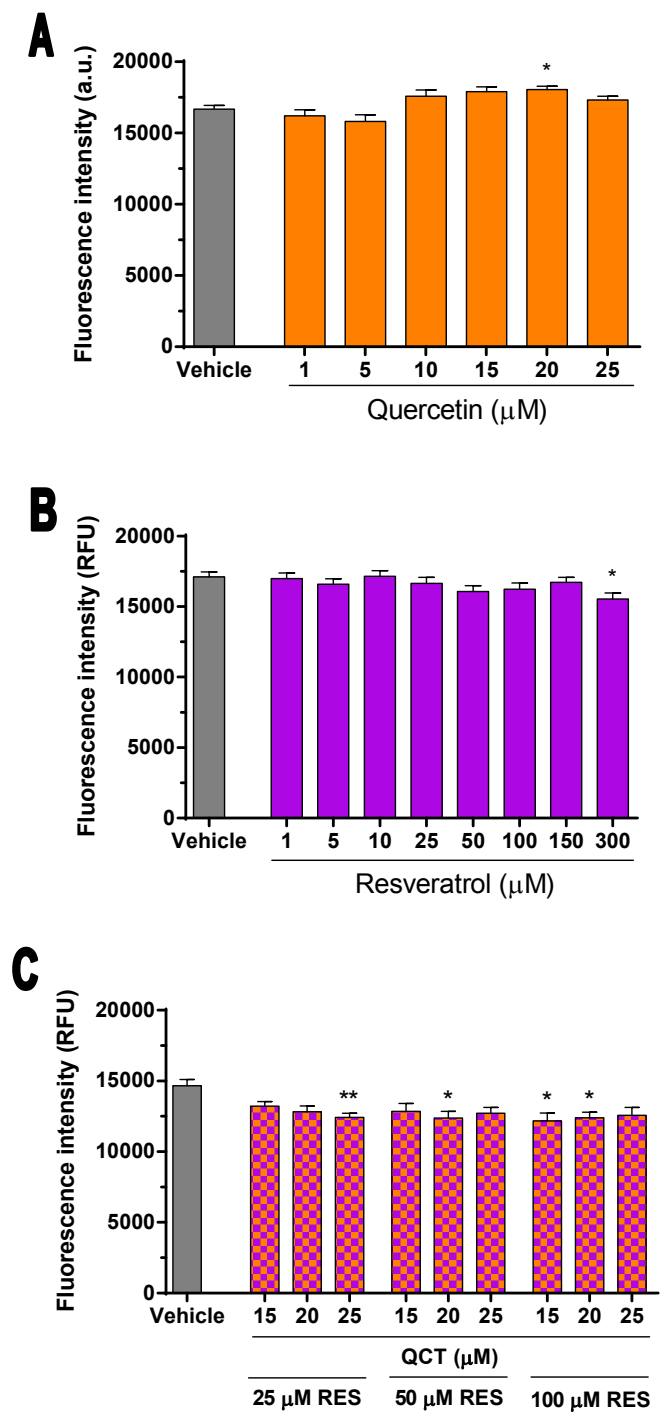

Figure 16. Cytotoxicity effect of quercetin (QCT) (A), resveratrol (RES)

(B) and their combinations (C) on IOBA-NHC cell line. ${ }^{*} p<0.05,{ }^{* *} p<0.01$, compared to vehicle $(0.5 \%$ ethanol). $\mathrm{N}=3$. 


\section{VI.1.1.2. Cytotoxicity in HCE cells}

Figure $17(A-C)$ shows the fluorescence intensity for HCE cells treated with QCT, RES and QCT+RES. QCT did not affect HCE cell viability at any concentration tested (1, $510,15,20$ and $25 \mu \mathrm{M})$, compared to vehicle (Figure 17A). When HCE cells were treated with RES $(1,5,10,25,50,100$ and $300 \mu \mathrm{M})$, cell viability only decreased at $300 \mu \mathrm{M}$ RES ( $p<0.001$ ), compared to vehicle (Figure 17B). No combination of 15, 20 and $25 \mu \mathrm{M}$ QCT with each of 25, 50 and $100 \mu \mathrm{M}$ RES was toxic for HCE cells (Figure $17 C)$.
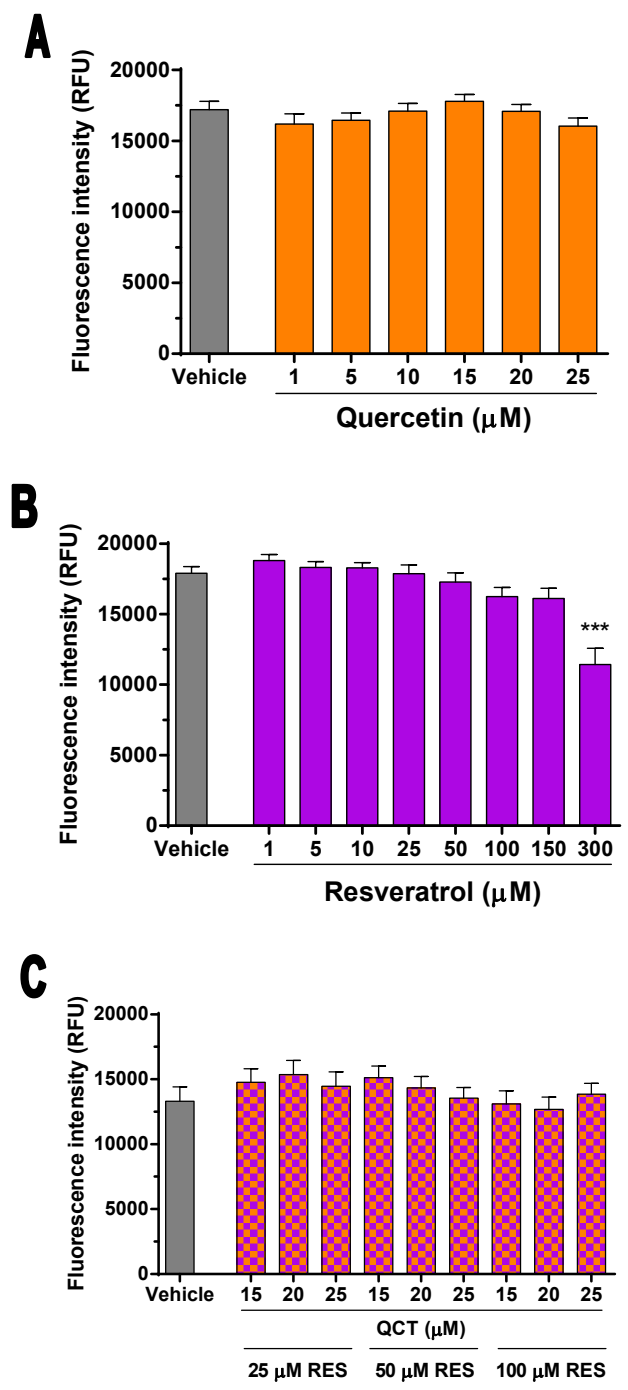

Figure 17. Cytotoxicity effect of quercetin (QCT) (A), resveratrol (RES)

(B) and their combinations (C) on HCE cell line. ${ }^{* * *} p<0.001$, compared to vehicle $(0.5 \%$ ethanol). $\mathrm{N}=3$. 


\section{VI.1.2. Effect of Quercetin and Resveratrol on cytokine/chemokine secretion induced by TNF- $\alpha$}

After determining the non-toxic concentrations of QCT, RES and their combinations, the anti-inflammatory effect of polyphenols were studied. For this purpose, secreted IL-6, IL-8/CXCL8, IP-10/CXCL10 and VEGF dose-response curves were obtained for QCT $(0.5,1,5,10,15,20$ and $25 \mu \mathrm{M})$ and $\operatorname{RES}(0.5,1,5,10,25$ and $50 \mu \mathrm{M}$ ) in conjunctival and corneal epithelial cells stimulated with $25 \mathrm{ng} / \mathrm{mL}$ TNF- $\alpha$.

After that, two concentrations of polyphenols (0.5 $\mu \mathrm{M}$ QCT and $5 \mu \mathrm{M}$ RES), which did not have a significant effect on cytokine/chemokine secretion in doseresponse curves, were chosen in order to determine the effect of both compounds together. Cytokine/chemokine secretion by both cell lines was determined using a multiplex-bead based array.

For a better comprehension of all data presented herein, results from polyphenols-treated IOBA-NHC cells are firstly presented, followed thereafter by the results from polyphenols-treated HCE cells. For each cell type, results are also subdivided into four sections, according to the cytokines under study: I) IL-6, II) IL8/CXCL8, III) IP-10/CXCL10 and IV) VEGF.

\section{VI.1.2.1. Cytokine/chemokine secretion by IOBA-NHC cells}

\section{VI.1.2.1.1. IL-6 secretion}

Figure 18 (A-C) shows the effect of QCT, RES and their combination on IL-6 secretion by TNF- $\alpha$-stimulated IOBA-NHC cells. Stimulation of IOBA-NHC cells with TNF- $\alpha$ induced a significant increase of IL-6 ( $p<0.01)$, compared to control cells. This TNF- $\alpha$-induced IL-6 secretion decreased in a dose-dependent manner when IOBANHC cells were treated with 15,20 and $25 \mu \mathrm{M}$ QCT $(p<0.05 ; p<0.01$ and $p<0.01$, respectively; Figure 18A), reducing IL-6 to almost basal levels. There was also a decrease in TNF- $\alpha$-induced IL-6 secretion when IOBA-NHC cells were treated with 25 and $50 \mu \mathrm{M}$ RES ( $p<0.05$ and $p<0.01$, respectively, Figure 18B). When $0.5 \mu \mathrm{M} Q C T$ and $5 \mu \mathrm{M}$ RES were mixed and tested in IOBA-NHC cells, there was an inhibitory effect of $0.5 \mu \mathrm{M} Q C T+5 \mu \mathrm{M}$ RES ( $\mathrm{p}<0.01$ ), which was stronger than that observed for $0.5 \mu \mathrm{M}$ QCT $(p<0.05)$ and $5 \mu M$ RES $(p>0.05)$ individually (Figure $18 C)$. 
A

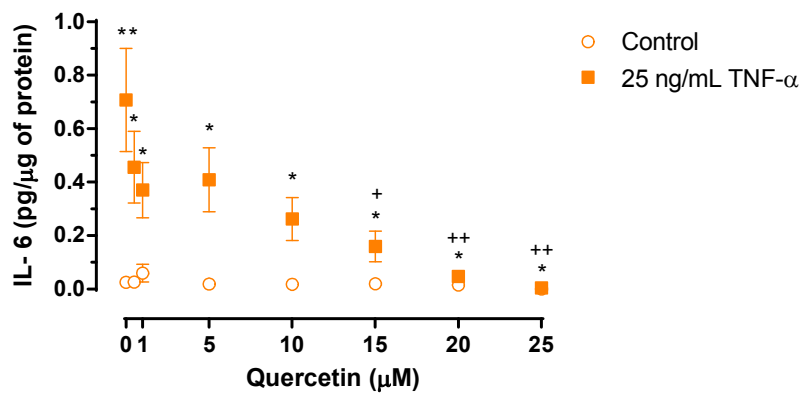

B

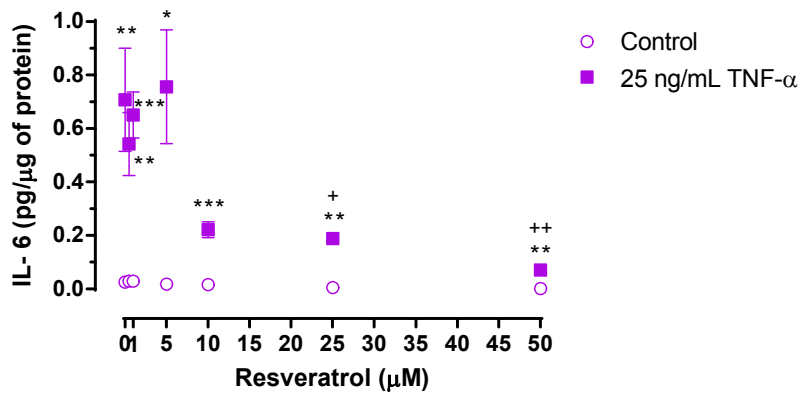

C

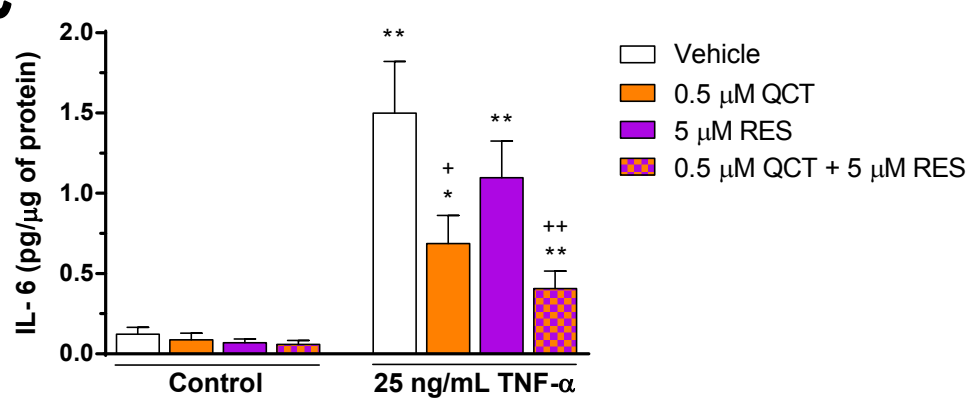

Figure 18. Effect of quercetin (QCT) (A), resveratrol (RES) (B) and their combination (C) on IL- 6 secretion by IOBA-NHC cells. ${ }^{*} p<0.05,{ }^{* *} p<0.01,{ }^{* * *} p<0.001$, compared to control cells; ${ }^{+} p<0.05,{ }^{++} p<0.01$, compared to vehicle-treated stimulated cells $(0.5 \%$ ethanol). $\mathrm{N}=3$.

\section{VI.1.2.1.2. IL-8/CXCL8 secretion}

Figure 19 (A-C) shows the effect of QCT, RES and their combination on IL8/CXCL8 secretion by TNF- $\alpha$-stimulated IOBA-NHC cells. Stimulation of IOBA-NHC cells with TNF- $\alpha$ induced a significant increase of IL-8/CXCL8 $(p<0.001)$, compared to control cells. When cells were treated with polyphenols, this stimulated secretion of IL8/CXCL8 decreased at 20 and $25 \mu \mathrm{M} Q C T$ ( $p<0.05$ and $p<0.01$, respectively, Figure 19A), whilst RES treatment decreased TNF- $\alpha$-induced IL-8/CXCL8 secretion only at 50 $\mu \mathrm{M}(\mathrm{p}<0.05$; Figure 19B). The mix of both compounds also significantly decreased IL- 
8/CXCL8 production $(p<0.05)$, but this decrease was similar to that found in cells treated with $0.5 \mu \mathrm{M} Q C T(p<0.05$; Figure 19C).
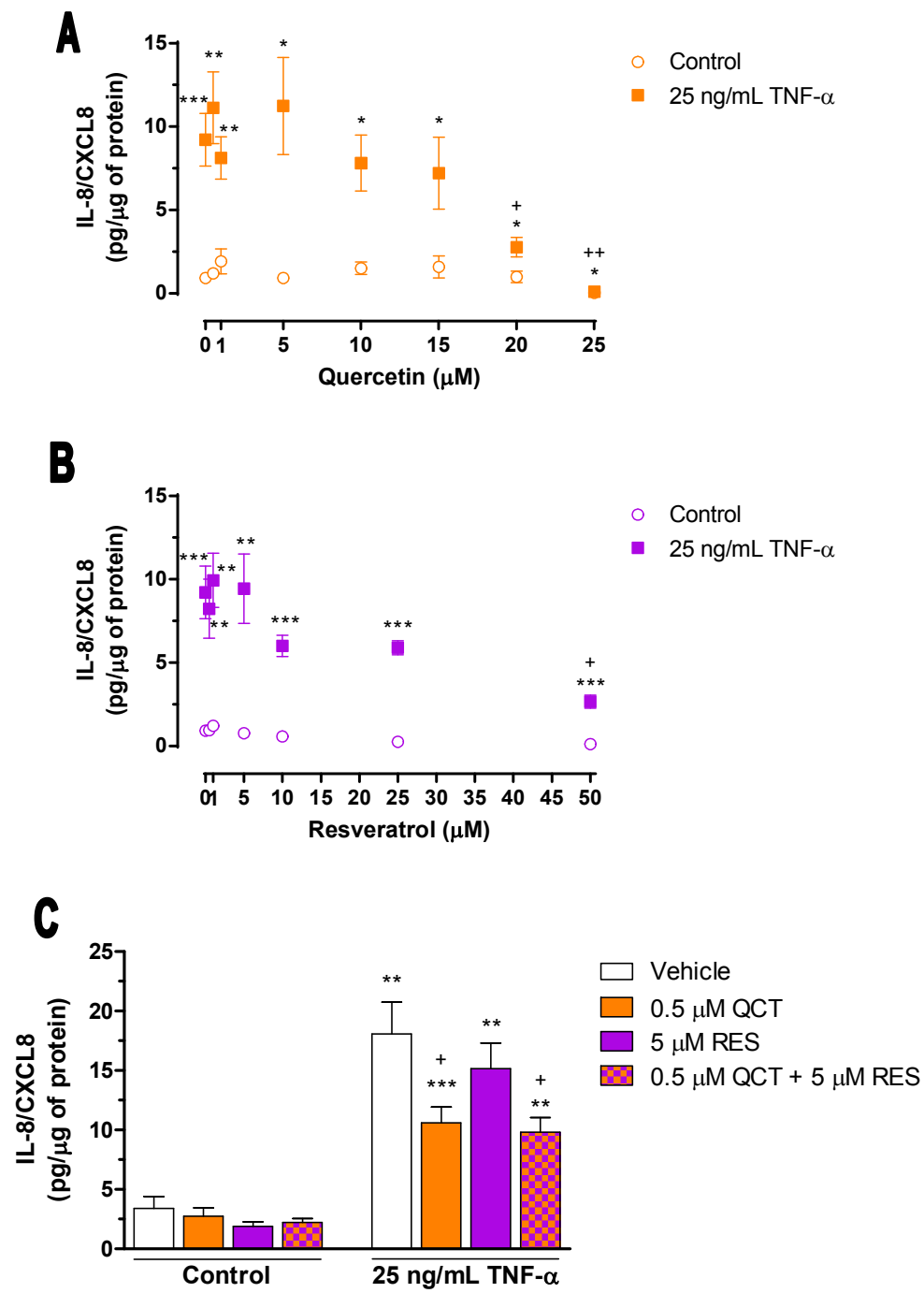

Figure 19. Effect of quercetin (QCT) (A), resveratrol (RES) (B) and their combination (C) on IL-8/CXCL8 secretion by IOBANHC cells. ${ }^{*} p<0.05,{ }^{* *} p<0.01,{ }^{* * *} p<0.001$, compared to control cells; ${ }^{+} p<0.05,{ }^{++} p<0.01$, compared to vehicle-treated stimulated cells $(0.5 \%$ ethanol). $\mathrm{N}=3$.

\section{VI.1.2.1.3. IP-10/CXCL10 secretion}

The effect of QCT, RES and QCT+RES on IP-10/CXCL10 secreted by TNF- $\alpha-$ treated IOBA-NHC cells is shown in Figure 20 (A-C). TNF- $\alpha$ provoked an increase of IP-10/CXCL10 secretion $(p<0.01)$. As shown in Figure $20 A$, this increase of IP10/CXCL10 secretion was attenuated by $10 \mu \mathrm{M} Q \mathrm{QCT}$ ( $p<0.01$ ), and decreased to basal levels at 15,20 and $25 \mu \mathrm{M}$ QCT (all $p<0.01$, compared to TNF- $\alpha$-stimulated cells). RES 
decreased IP-10/CXCL10 at concentrations of 10, 25 and $50 \mu \mathrm{M}$ (all $p<0.05$ ) compared to stimulated cells (Figure 20B), but not down to basal levels. No significant differences in IP-10/CXCL10 levels were found when IOBA-NHC cells were treated with $0.5 \mu \mathrm{M}$ $\mathrm{QCT}+5 \mu \mathrm{M}$ RES with respect to stimulated cells (Figure 20C).
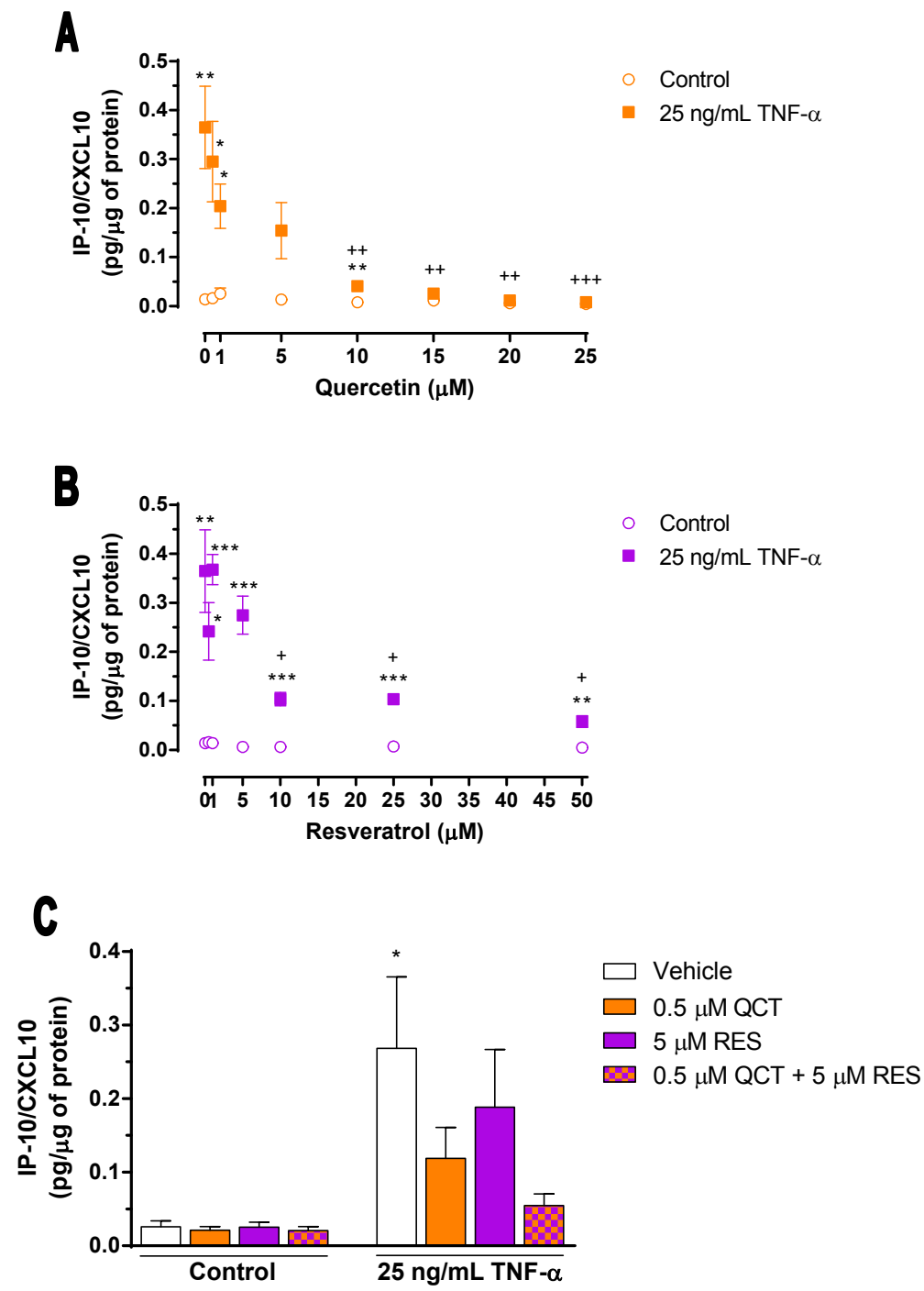

Figure 20. Effect of quercetin (QCT) (A), resveratrol (RES) (B) and their combination (C) on IP-10/CXCL10 secretion by IOBANHC cells. ${ }^{*} p<0.05,{ }^{* *} p<0.01,{ }^{* * *} p<0.001$, compared to control cells; ${ }^{+} p<0.05,{ }^{++} p<0.01,{ }^{+++} p<0.001$, compared to vehicle-treated stimulated cells $(0.5 \%$ ethanol). $\mathrm{N}=3$. 


\section{VI.1.2.1.4. VEGF secretion}

Figure $21(\mathrm{~A}-\mathrm{C}$ ) shows the effect of QCT, RES and $0.5 \mu \mathrm{M}$ QCT + $5 \mu \mathrm{M}$ RES on VEGF secretion by IOBA-NHC cells. TNF- $\alpha$ did not induce VEGF production by IOBA$\mathrm{NHC}$ cells. None of the QCT and RES concentrations had a significant effect on VEGF secretion in dose-response curves (Figure $21 \mathrm{~A}-\mathrm{B}$ ). Although $0.5 \mu \mathrm{M} \mathrm{QCT}$ showed a lower level of VEGF secretion (but not significant compared to control, Figure 21A), it was significant $(p<0.01)$ when the QCT+RES combination was further tested, as shown in Figure 21C. In addition, the combination $0.5 \mu \mathrm{M}$ QCT + $5 \mu \mathrm{M}$ RES decreased VEGF secretion in TNF- $\alpha$-stimulated conjunctival epithelial cells $(p<0.01)$, compared to vehicle-treated stimulated cells (Figure 21C).

A

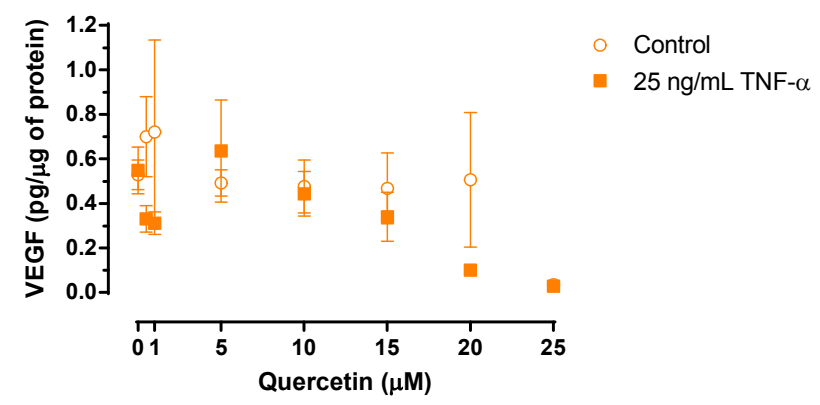

B


Figure 21. Effect of quercetin (QCT) (A), resveratrol (RES) (B) and their combination (C) on VEGF secretion by IOBA-NHC cells. ${ }^{* *} p<0.01$, compared to control cells; ${ }^{++} p<0.01$, compared to vehicle-treated stimulated cells $(0.5 \%$ ethanol). $\mathrm{N}=3$. 


\section{VI.1.2.2. Cytokine/chemokine secretion by HCE cells}

\section{VI.1.2.2.1. IL-6 secretion}

The effects of QCT, RES and their combination on IL-6 secretion by HCE cells are shown in Figure 22 (A-C). In these cells, TNF- $\alpha$ induced a significant increase of IL6 secretion $(p<0.001)$. When TNF- $\alpha$-stimulated cells were treated with $1 \mu \mathrm{M} Q C T$, IL-6 secretion was significantly decreased $(p<0.01)$. Moreover, concentrations of $5,10,15$, 20 and $25 \mu \mathrm{M}$ QCT strongly reduced IL-6 production, with all concentrations showing similar inhibitory effects (all $p<0.001$; Figure 22A). There was a decrease of IL-6 secretion in a dose-dependent manner when HCE cells were treated with 10, 25 and $50 \mu \mathrm{M}$ RES (all $p<0.001$; Figure 22B). However, the combination of QCT and RES did not have a significant effect on TNF-a-induced IL-6 secretion (Figure 22C).

A

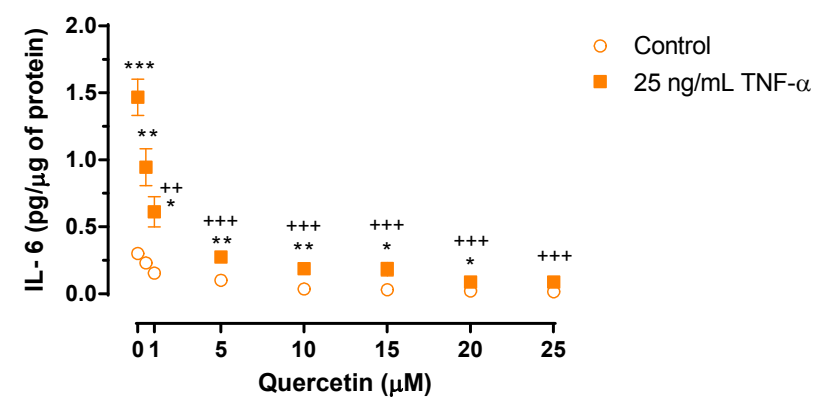

B
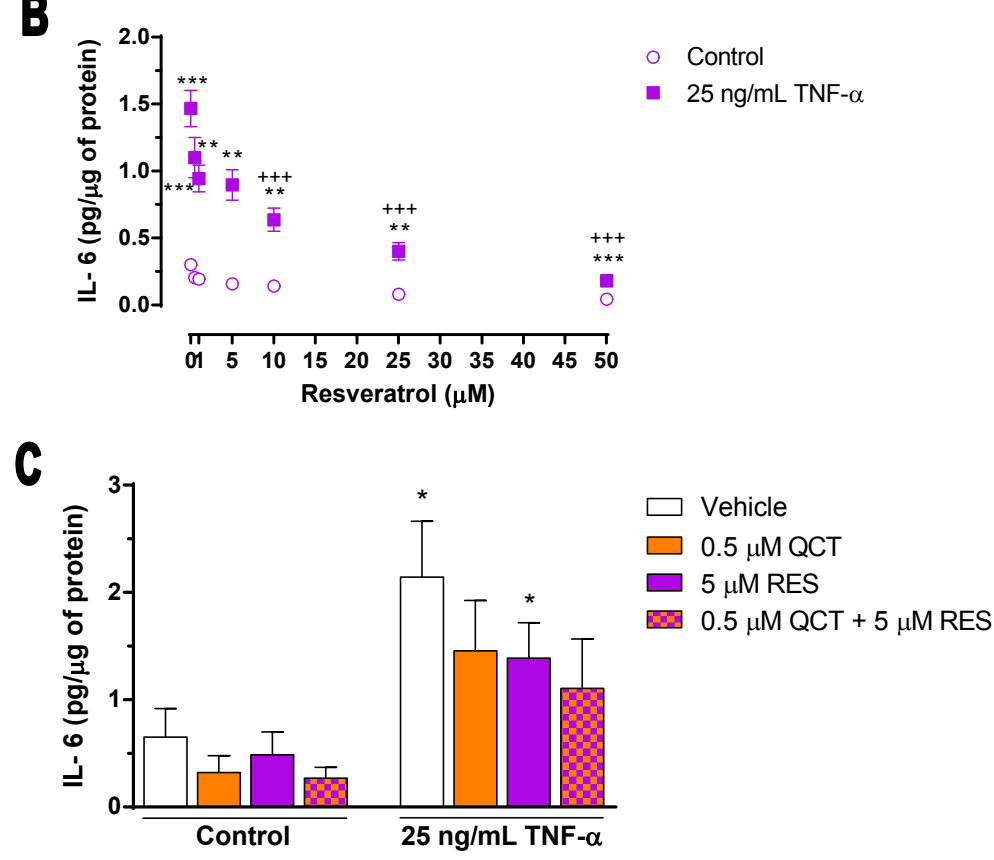

Figure 22. Effect of quercetin (QCT) (A), resveratrol (RES) (B) and their combination (C) on IL- 6 secretion by HCE cells. ${ }^{*} p<0.05$, ${ }^{* *} p<0.01,{ }^{* * *} p<0.001$, compared to control cells; ${ }^{++} p<0.01$, ${ }^{+++} p<0.001$, compared to vehicle-treated stimulated cells $(0.5 \%$ ethanol). $\mathrm{N}=3$. 


\section{VI.1.2.2.2. IL-8/CXCL8 secretion}

The effect of QCT, RES and their combination on IL-8/CXCL8 secretion by TNF- $\alpha$-stimulated HCE cells is shown in Figure 23 (A-C). Stimulation of HCE cells with TNF- $\alpha$ induced a significant increase of IL-8/CXCL8 chemokine $(p<0.001)$. When HCE cells were treated with polyphenols, TNF- $\alpha$-stimulated IL-8/CXCL8 secretion was significantly decreased at $5 \mu \mathrm{M} Q C T(p<0.05)$. This effect was found to be stronger at $10,15,20$ and $25 \mu \mathrm{M}$ QCT $(p<0.01$; Figure 23A). Different results were found for RES treatments, because only the highest concentration used $(50 \mu \mathrm{M})$ significantly decreased IL-8/CXCL8 secretion ( $p<0.05$; Figure 23B). The mix of both compounds did not have any effect on TNF- $\alpha$-stimulated IL-8/CXCL8 secretion (Figure 23C).
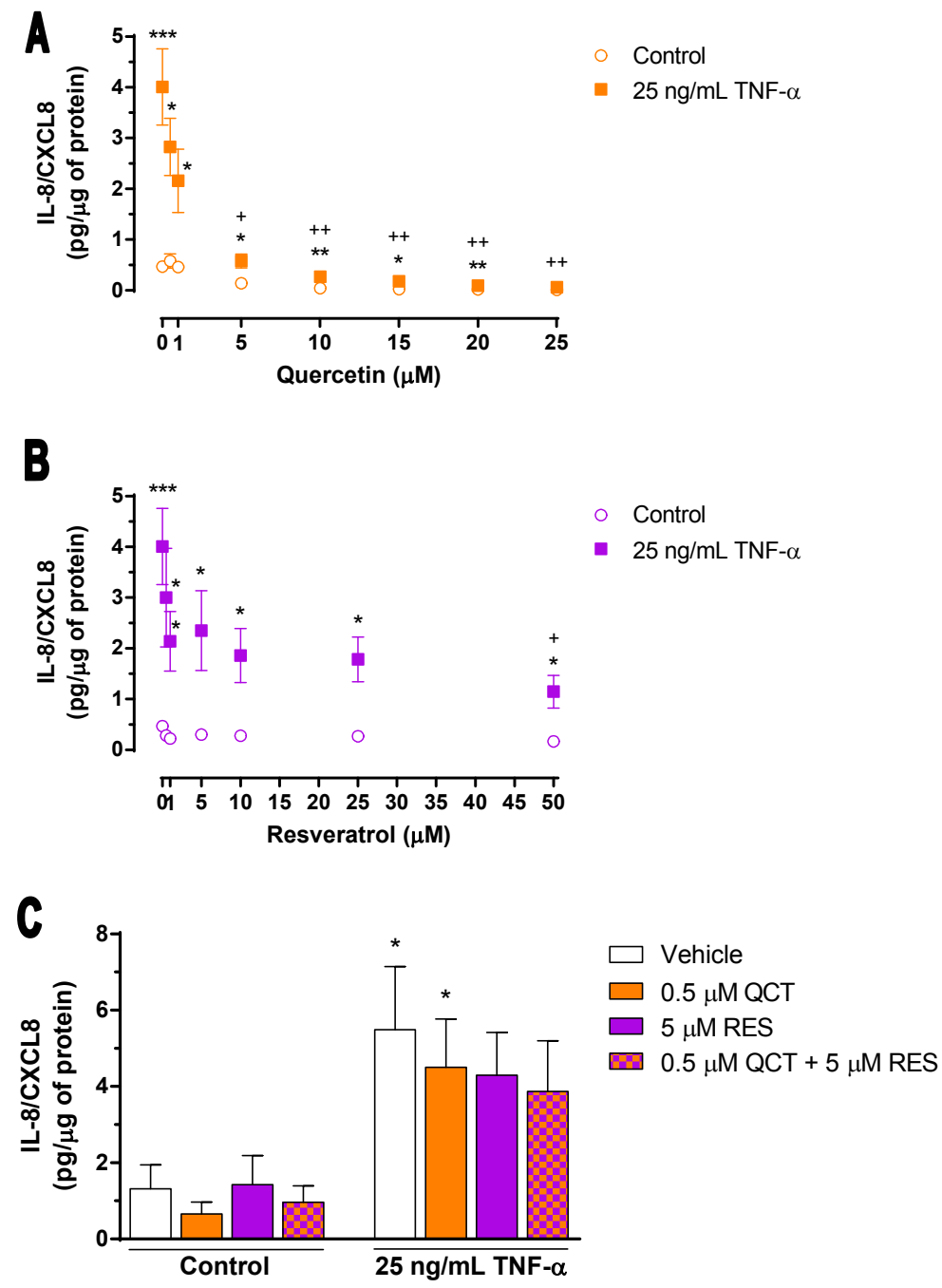

Figure 23. Effect of quercetin (QCT) (A), resveratrol (RES) (B) and their combination (C) on IL-8/CXCL8 secretion by HCE cells. ${ }^{*} p<0.05$, ${ }^{* *} p<0.01,{ }^{* * *} p<0.001$, compared to control cells; ${ }^{+} p<0.05,{ }^{++} p<0.01$, compared to vehicle-treated stimulated cells ( $0.5 \%$ ethanol). $\mathrm{N}=3$. 


\section{VI.1.2.2.3. IP-10/CXCL10 secretion}

Figure $24(\mathrm{~A}-\mathrm{C})$ shows the effect of QCT, RES and their combination on TNF- $\alpha-$ induced IP-10/CXCL10 secretion by HCE cells. TNF- $\alpha$ provoked an increase of IP10/CXCL10 secretion $(p<0.01)$. As shown in Figure 24A, IP-10/CXCL10 secretion by TNF- $\alpha$-stimulated HCE cells was attenuated by QCT at $1 \mu \mathrm{M}(\mathrm{p}<0.05)$, and by 5,10 , 15,20 and $25 \mu \mathrm{M} Q \mathrm{QCT}$, which significantly (all $p<0.001$ ) reduced chemokine secretion down to unstimulated levels. RES decreased IP-10/CXCL10 secretion stimulated by TNF- $\alpha$ at all concentrations tested: 0.5 and $1 \mu \mathrm{M}(\mathrm{p}<0.05)$, and 5, 10, 25 and $50 \mu \mathrm{M}$ $(p<0.01$ ); however only $50 \mu \mathrm{M}$ RES decreased IP-10/CXCL10 to basal levels (Figure 24B). No significant differences in IP-10/CXCL10 levels were found when IOBA-NHC cells were treated with $0.5 \mu \mathrm{M}$ QCT $+5 \mu \mathrm{M}$ RES (Figure 24C).


Figure 24. Effect of quercetin (QCT) (A), resveratrol (RES) (B) and their combination (C) on IP-10/CXCL10 secretion by HCE cells. ${ }^{*} p<0.05,{ }^{* *} p<0.01,{ }^{* * *} p<0.001$, compared to control cells; ${ }^{+} p<0.05$, ${ }^{++} \mathrm{p}<0.01,{ }^{++} \mathrm{p}<0.001$, compared to vehicle-treated stimulated cells ( $0.5 \%$ ethanol). $\mathrm{N}=3$. 


\section{VI.1.2.2.4. VEGF secretion}

Figure $25(\mathrm{~A}-\mathrm{C})$ shows the effect of QCT, RES and $0.5 \mu \mathrm{M} \mathrm{QCT}+5 \mu \mathrm{M}$ RES on VEGF secretion by HCE cells. TNF- $\alpha$ did not induce VEGF production in HCE cells. Neither QCT, nor RES, nor their combination had an effect on VEGF secretion by corneal epithelial cells (Figures $25 \mathrm{~A}, \mathrm{~B}$ and C).

A



B

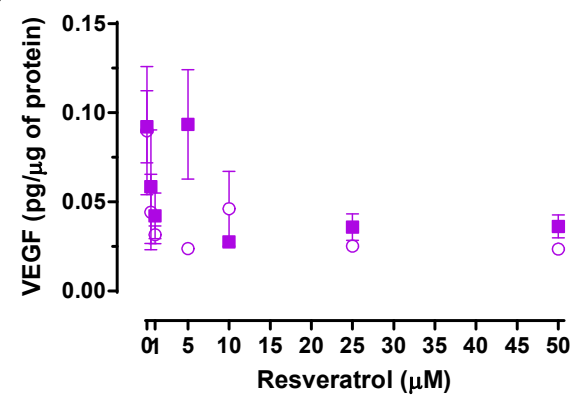

○ Control

- $25 \mathrm{ng} / \mathrm{mL}$ TNF- $\alpha$

$c$

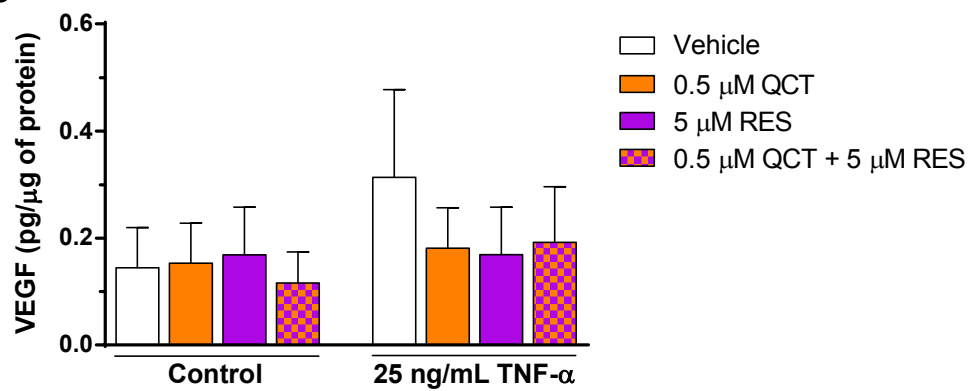

Figure 25. Effect of quercetin (QCT) (A), resveratrol (RES) (B) and their combination (C) on VEGF secretion by HCE cells. $\mathrm{N}=3$. 


\section{VI.1.3. Effect of Quercetin and Resveratrol on COX-2 expression}

The effect of QCT and RES on COX-2 expression was examined in order to investigate their underlying mechanisms on TNF-a-induced inflammation in both epithelial cell lines. In the present study, both IOBA-NHC and HCE cells were stimulated with $25 \mathrm{ng} / \mathrm{mL}$ TNF- $\alpha$ and treated with 0.5 and $25 \mu \mathrm{M} \mathrm{QCT,} 5$ and $50 \mu \mathrm{M}$ RES, and the polyphenol mixture $(0.5 \mu \mathrm{M}$ QCT + $5 \mu \mathrm{M}$ RES). COX-2 expression was analysed by SDS-PAGE Western blotting. All data were normalised to GAPDH expression.

\section{VI.1.3.1. COX-2 expression by IOBA-NHC cells}

Figure 26A shows the effect of polyphenols on TNF- $\alpha$-induced COX-2 expression by IOBA-NHC cells, normalised to GAPDH expression. TNF- $\alpha$ caused an increase of COX-2 expression ( $p<0.001$ ), compared to unstimulated cells. None of the polyphenol treatments had any effect on COX-2 expression, but $25 \mu \mathrm{M}$ QCT produced an increase of COX-2 expression in both unstimulated and stimulated cells. Data are also presented as fold-change (Figure 26B). TNF- $\alpha$ produced a 5-fold increase of COX2 expression. Both concentrations of RES ( 5 and $50 \mu \mathrm{M}$ ) produced a 4-fold decrease of COX-2 expression, whereas 0.5 and $25 \mu \mathrm{M}$ QCT induced a 3-fold and 2.5-fold decrease, respectively. The polyphenol mixture $(0.5 \mu \mathrm{M} \mathrm{QCT}+5 \mu \mathrm{M}$ RES) demonstrated a stronger decrease in COX-2 expression (2.3-fold) than both polyphenols alone. 

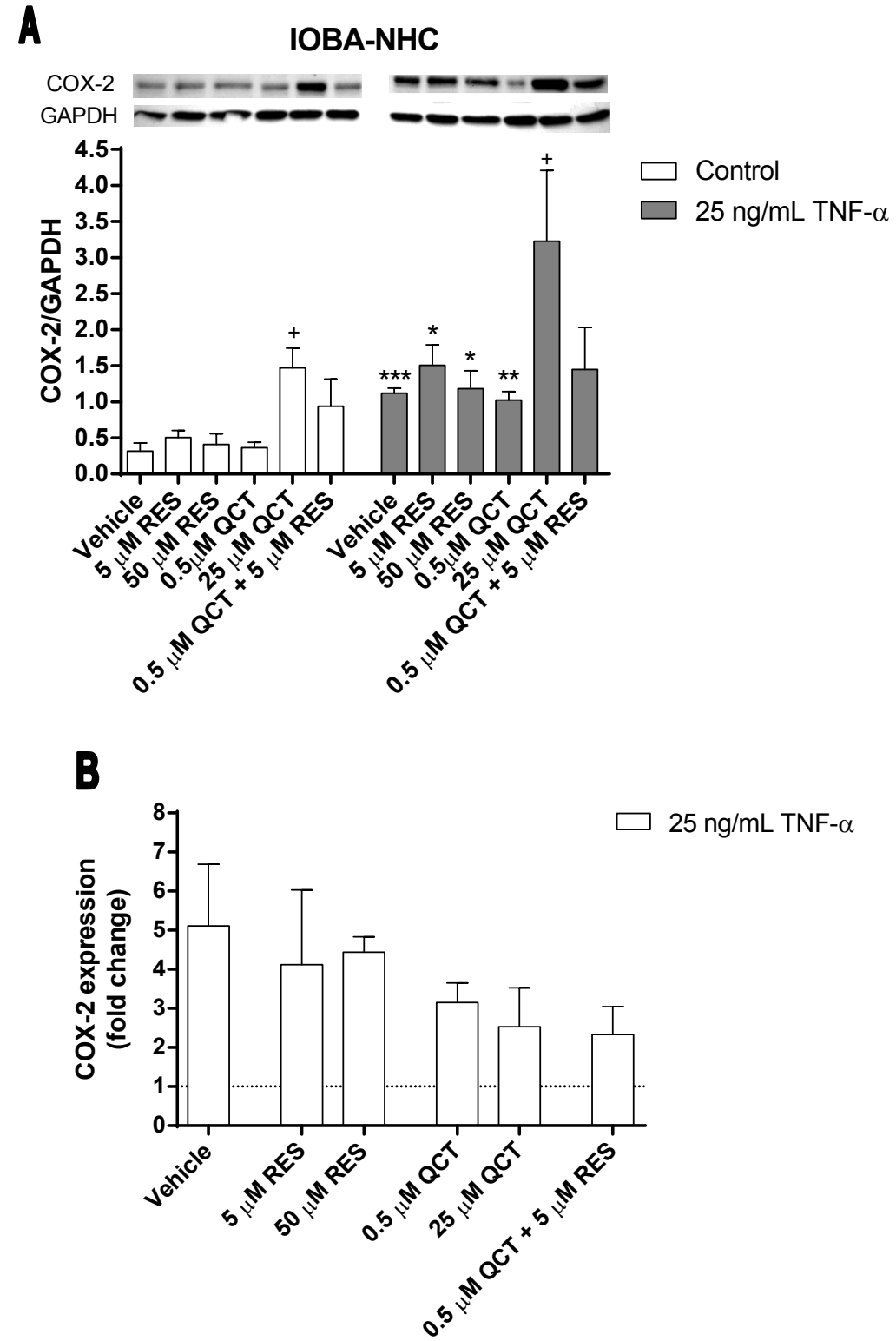

Figure 26. Effect of quercetin (QCT), resveratrol (RES) and their combination on COX-2 secretion by IOBA-NHC cells. ${ }^{*} p<0.05,{ }^{* *} p<0.01,{ }^{* * *} p<0.001$, compared to control cells; ${ }^{+} p<0.05$, compared to cells treated with vehicle $(0.5 \%$ ethanol). Dashed line represents control cells. A representative Western blot image is shown in Figure $A . N=2$.

\section{VI.1.3.2. COX-2 expression by HCE cells}

Figure 27A shows the effect of polyphenols on TNF- $\alpha$-induced COX-2 expression by HCE cells, normalised to GAPDH expression. TNF- $\alpha$ did not produce an increase of COX-2 expression. Neither RES treatments nor $0.5 \mu \mathrm{M} Q C T$ produced a significant decrease of COX-2 expression. Although $25 \mu \mathrm{M}$ QCT induced a significant decrease of COX-2 ( $p<0.05)$, compared to vehicle-treated stimulated cells. Treatment with $0.5 \mu \mathrm{M} \mathrm{QCT}+5 \mu \mathrm{M}$ RES did not affect significantly COX-2 expression. Data 
presented as fold-changes are shown in Figure 27B. TNF-a (vehicle) produced a 2-fold increase of COX-2 expression. Both RES treatments ( 5 and $50 \mu \mathrm{M})$ produced a 1 -fold decrease. The $0.5 \mu \mathrm{M}$ QCT concentration produced a 1.5-fold decrease, whilst $25 \mu \mathrm{M}$ QCT induced a 0.2 -fold decrease of COX-2 expression. The mix of polyphenols $(0.5$ $\mu \mathrm{M} Q \mathrm{QCT}+5 \mu \mathrm{M}$ RES) decreased COX-2 expression up to 0.4 -fold (Figure 27B).
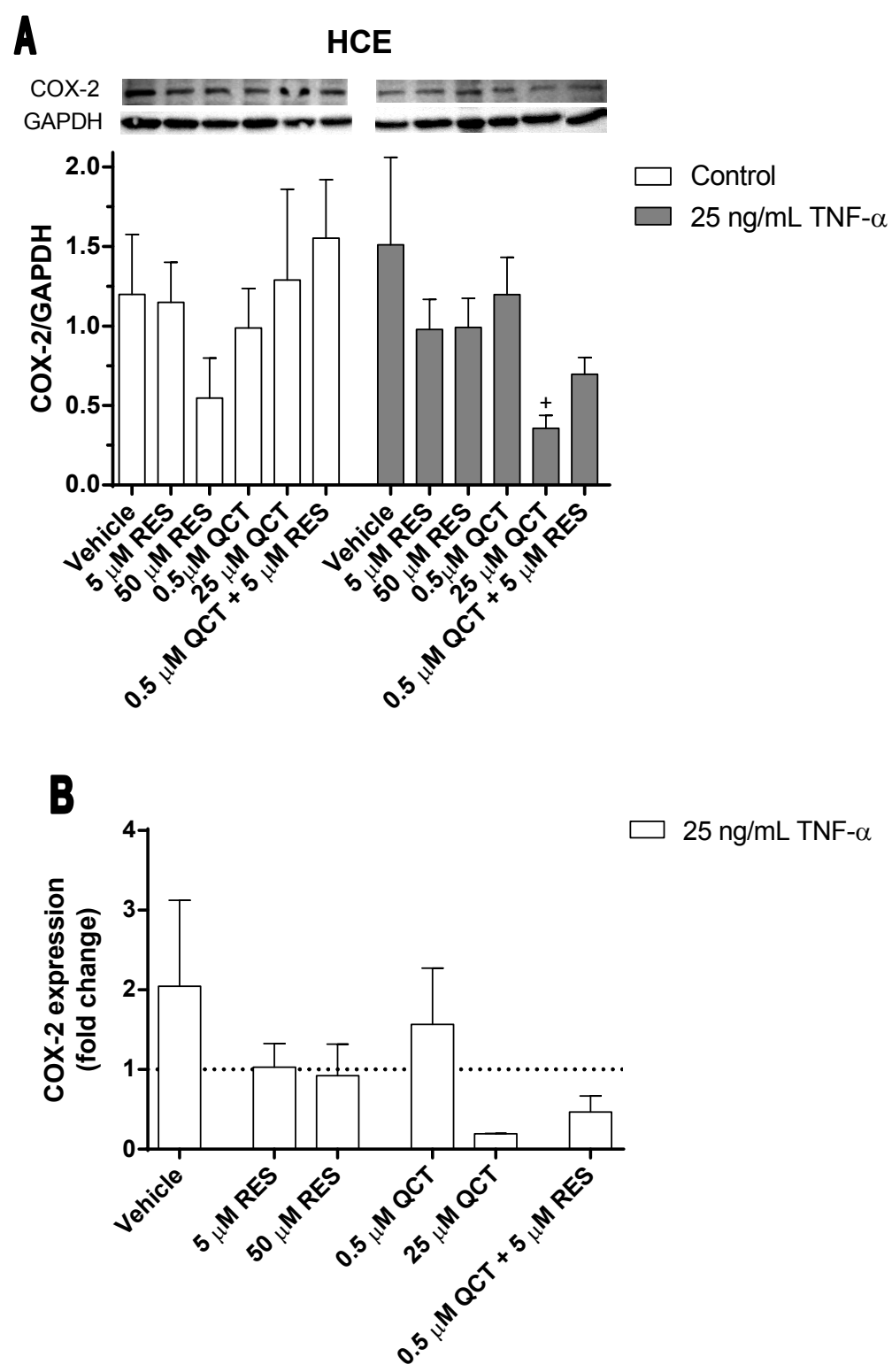

Figure 27. Effect of quercetin (QCT), resveratrol (RES) and their combination on COX-2 secretion by HCE cells. ${ }^{+} p<0.05$, compared to cells treated with vehicle $(0.5 \%$ ethanol). Dashed line represents control cells. A representative Western blot image is shown in Figure $A . N=2$. 


\section{VI.1.4. Reactive oxygen species induced by ultraviolet B radiation}

UV-B is the most common cause of radiation damage to the eye and is a contributor to oxidative damage in cells. As mentioned previously, QCT and RES are well known antioxidants. Moreover, both polyphenols are UV-absorbing compounds. Thus, dose-response curves were firstly obtained to study the effect of QCT and RES on UV-B-induced intracellular ROS, and a combination of low doses of both compounds was further analysed. As in previous sections within this "results chapter", data from IOBA-NHC cells are firstly presented, followed by results from HCE cells.

\section{VI.1.4.1. Intracellular ROS production by IOBA-NHC cells}

UV-B radiation produced a significant increase of intracellular ROS on IOBA$\mathrm{NHC}$ cells after $1 \mathrm{~h}(\mathrm{p}<0.01$; Figure 28). None of the concentrations tested of QCT (0.5, $1,5,10$ and $25 \mu \mathrm{M})$ significantly decreased intracellular ROS production in cells exposed to UV-B. It should be noted, however, that no significant differences were found between UV-B-exposed cells treated with 1, 5, 10 and $25 \mu \mathrm{M} \mathrm{QCT}$ and control cells (Figure 28A). When UV-B-exposed IOBA-NHC cells were treated with RES, only $50 \mu \mathrm{M}$ RES significantly decreased intracellular ROS production $(p<0.05$; Figure 28B). In addition, there were no significant differences between exposed and unexposed cells treated with 25 and $50 \mu \mathrm{M}$ RES (Figure 28B). Similar results were found when cells were treated with a combination of QCT and RES, although there were no significant differences between UV-B-exposed cells treated with vehicle and UV-Bexposed cells treated with $0.5 \mu \mathrm{M}$ QCT, $5 \mu \mathrm{M}$ RES or their combination. The latter (polyphenol combination) was not significantly different to its control (Figure 28C). 


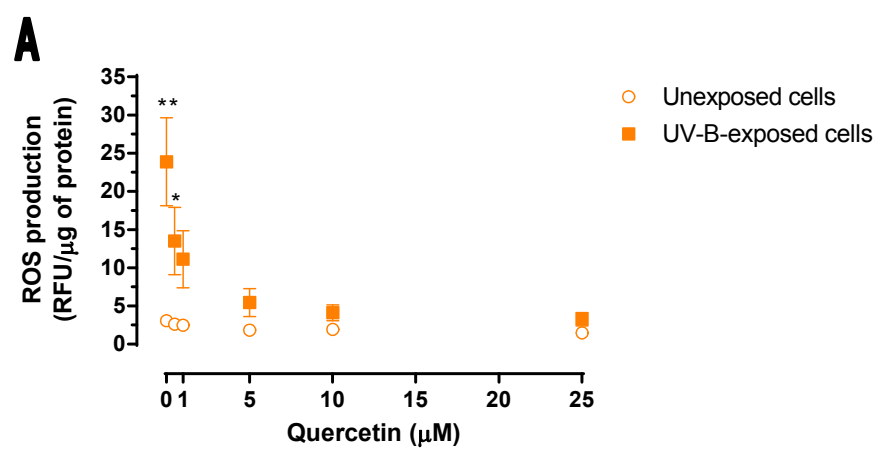

B

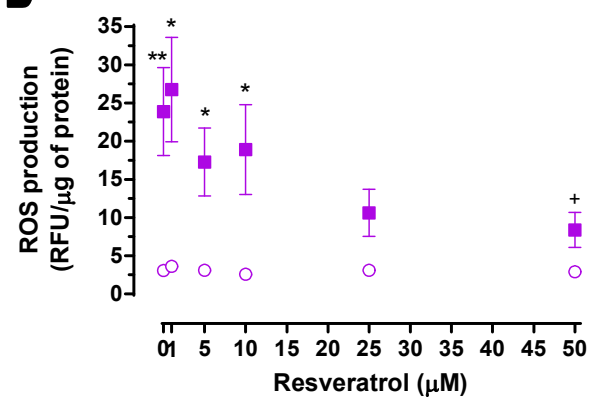

○ Unexposed cells

- UV-B-exposed cells

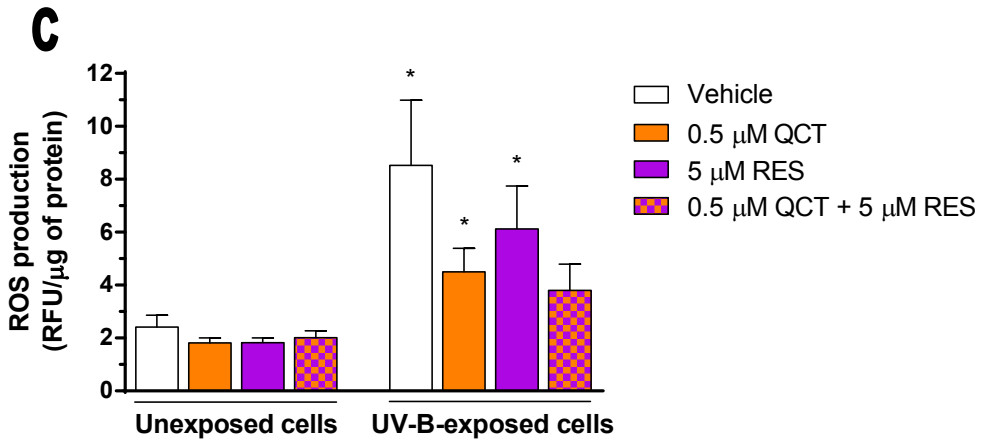

Figure 28. Effect of quercetin (QCT) (A), resveratrol (RES) (B) and their combination $(\mathrm{C})$ on intracellular reactive oxygen species (ROS) induced by UV-B in IOBA-NHC cells. ${ }^{*} p<0.05,{ }^{* *} p<0.01$, compared to unexposed cells; ${ }^{+} p<0.05$, compared to vehicle-treated UV-B-exposed cells $(0.5 \%$ ethanol). RFU: relative fluorescence units. $\mathrm{N}=3$.

\section{VI.1.4.2. Intracellular ROS production by HCE cells}

When HCE cells were irradiated with UV-B light, intracellular ROS increased dramatically ( $p<0.001$; Figure 29). All doses of QCT tested $(0.5,1,5,10$ and $25 \mu \mathrm{M}$ ) decreased intracellular ROS production significantly $(p<0.01, p<0.05, p<0.001, p<0.001$ and $p<0.001$, respectively; Figure 29A). Regarding RES treatments, 25 and $50 \mu \mathrm{M}$ RES decreased significantly UV-induced intracellular ROS production (all $p<0.001$ ), whilst lower concentrations of RES did not have any effect (Figure 29B). Similar results to those found in IOBA-NHC cells were found in HCE cells treated with a combination QCT and RES, although there was no significant difference between UV-B-exposed 
cells treated with $0.5 \mu \mathrm{M}$ QCT, $5 \mu \mathrm{M}$ RES or their combination and the vehicle-treated UV-B-exposed cells. The latter (polyphenol combination) was not significantly different to its control (Figure 29C).
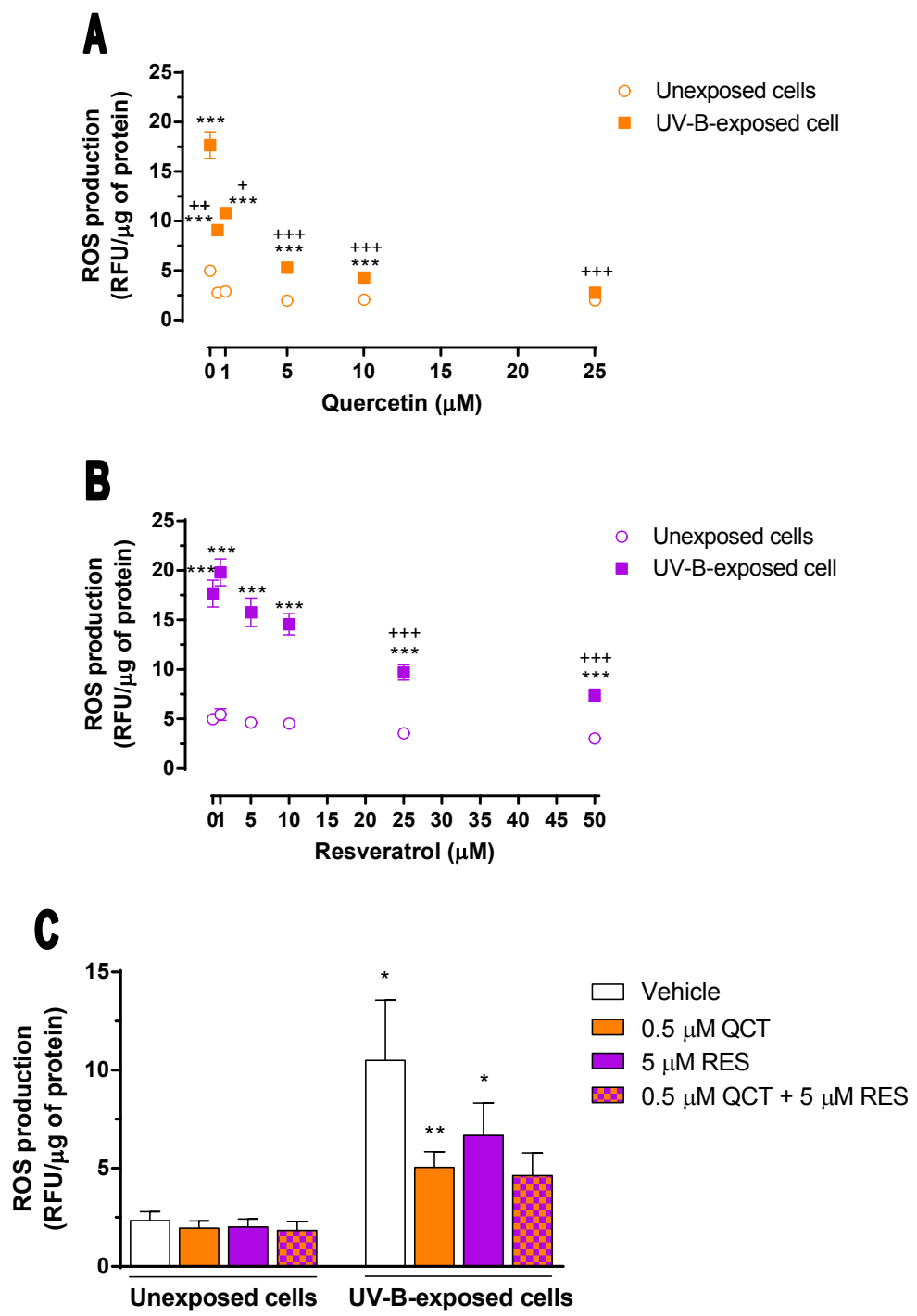

Figure 29. Effect of quercetin (QCT) (A), resveratrol (RES) (B) and their combination (C) on intracellular reactive oxygen species (ROS) induced by UV-B in HCE cells. ${ }^{*} p<0.05$, ${ }^{* *} p<0.01$, compared to unexposed cells; ${ }^{+} p<0.05,{ }^{++} p<0.01$, ${ }^{++} p<0.001$, compared to vehicle-treated UV-B-exposed cells ( $0.5 \%$ ethanol). RFU: relative fluorescence units. $\mathrm{N}=3$. 


\section{VI.2. In-vitro proliferative response of human T cells}

This section summarises all of the data obtained from testing the immunoregulatory effect of polyphenols on PBMCs from healthy donors.

\section{VI.2.1. Cytotoxicity of polyphenols}

The cytotoxicity of QCT, RES and QCT+RES in PBMCs stimulated with antiCD3/anti-CD28 after $96 \mathrm{~h}$ exposure was tested using PI staining and flow cytometry.

Figure 30 summarises all of the data from this assay. Polyphenol concentrations used in this experiment were: 0.5, 1, 5, 10, 15, 20 and $25 \mu \mathrm{M} Q C T ; 0.5$, $1,5,10,25,50$ and $100 \mu \mathrm{M}$ RES; and the mix of all concentrations of QCT with all concentrations of RES.

PBMCs stimulated with anti-CD3/anti-CD28 antibodies increased significantly viability compared to unstimulated cells ( $p<0.001$; Figure 30 ).

As it has been pointed out in "material and methods" section, polyphenol treatments have different concentrations of vehicle depending on whether QCT and RES were added separately $(0.5 \% \mathrm{EtOH})$ or mixed $(1 \% \mathrm{EtOH})$. However, there was not a significant difference in PBMC viability between either concentrations of $\mathrm{EtOH}$ in culture medium (Figure 30).

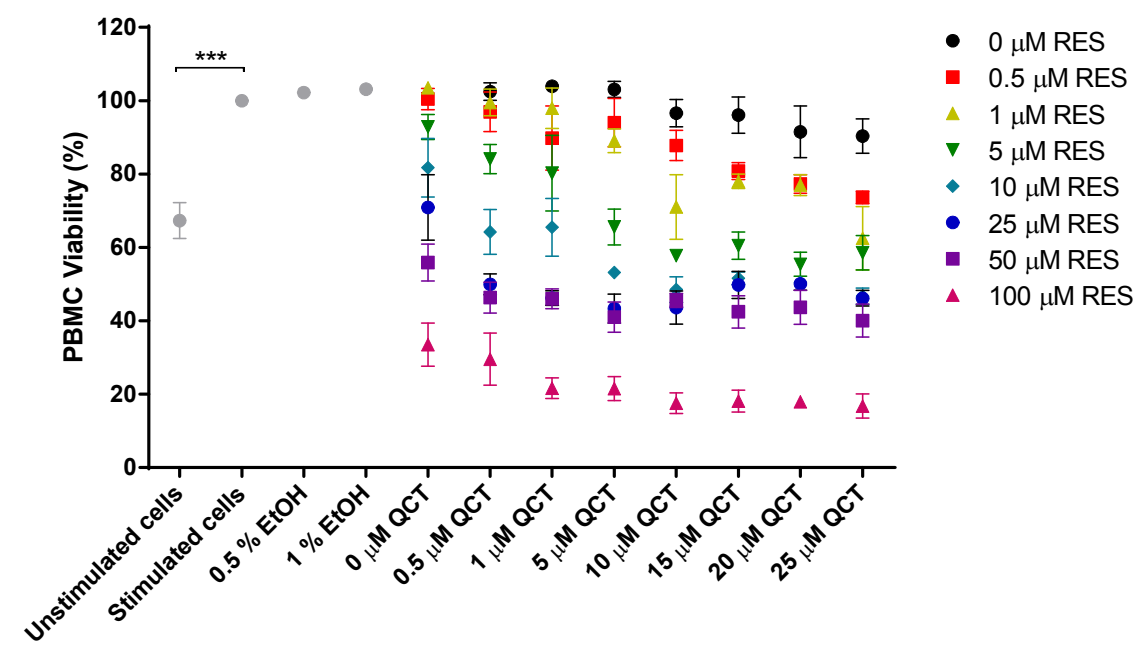

Figure 30. Cytotoxicity of quercetin (QCT), resveratrol (RES) and their combinations in peripheral blood mononuclear cells (PBMCs). ${ }^{* * *} p<0.001$. EtOH: ethanol. $\mathrm{N}=3$.

In order to clarify comparisons between polyphenol treatments, the cytotoxicity data are subdivided as follows: i) cytotoxicity of QCT, ii) cytotoxicity of RES and iii) cytotoxicity of combinations. 


\section{VI.2.1.1. Cytotoxicity of QCT}

Figure 31 shows cytotoxicity of QCT in PBMCs. Seven concentrations of QCT were tested: $0.5,1,5,10,15,20$ and $25 \mu \mathrm{M}$. None of the QCT concentrations used were toxic for PBMCs, after $96 \mathrm{~h}$ of culture.

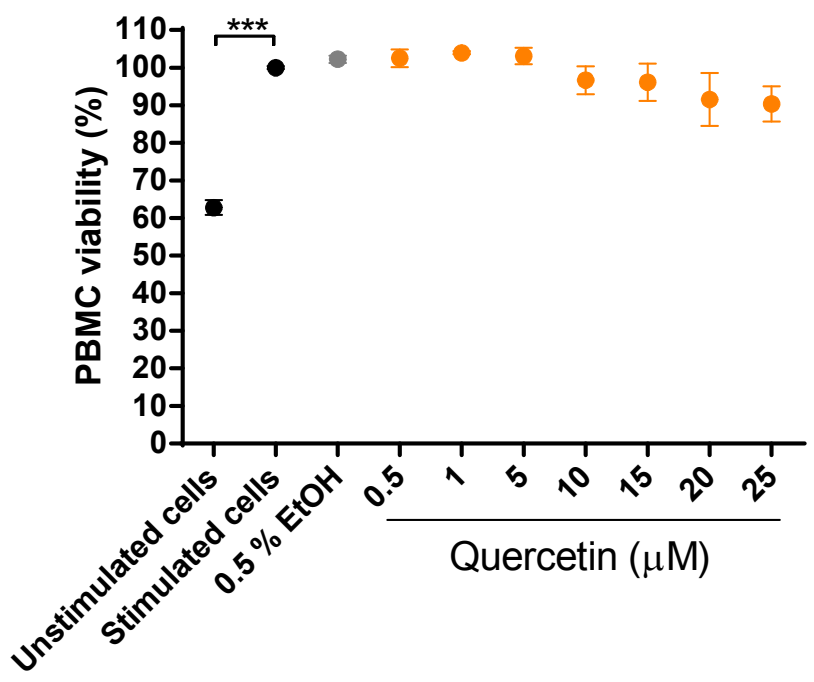

Figure 31. Cytotoxicity of quercetin (QCT) in peripheral blood mononuclear cells (PBMCs). ${ }^{* * *} \mathrm{p}<0.001$. EtOH: ethanol. $\mathrm{N}=3$.

\section{VI.2.1.2. Cytotoxicity of RES}

Figure 32 shows the cytotoxicity of RES in PBMCs. Seven concentrations of RES were tested: $0.5,1,5,10,25,50$ and $100 \mu \mathrm{M}$. High concentrations of RES (50 and $100 \mu \mathrm{M}$ ) significantly decreased ( $p<0.05$ and $p<0.01$, respectively) the percentage of viable cells.

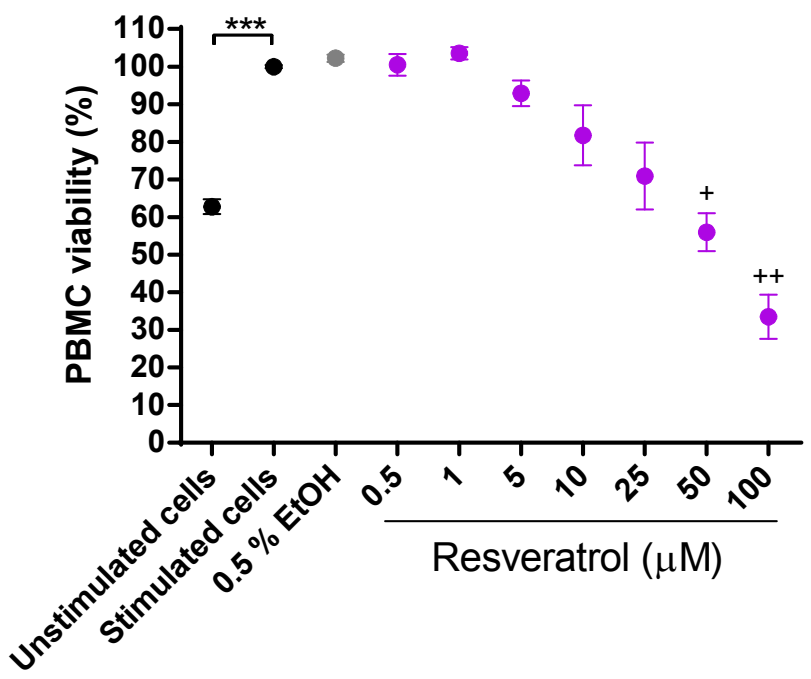

Figure 32. Cytotoxicity of resveratrol (RES) in peripheral blood mononuclear cells (PBMCs). ${ }^{* * *} p<0.001 .{ }^{+} p<0.05,{ }^{++} p<0.01$, compared to PBMCs treated with $0.5 \%$ ethanol $(\mathrm{EtOH}) . \mathrm{N}=3$. 


\section{VI.2.1.3. Cytotoxicity of combination of QCT and RES}

Figure 33 shows the cytotoxicity of combinations of QCT and RES in PBMCs and Table 7 shows significant differences for each combination of polyphenols, compared to stimulated cells treated with $\mathrm{EtOH}$. Seven concentrations of QCT $(0.5,1$, $5,10,15,20$ and $25 \mu \mathrm{M})$ and seven concentrations of RES $(0.5,1,5,10,25,50$ and $100 \mu \mathrm{M}$ ) were tested alone and in combination with each other. Cell viability decreased when QCT and RES concentrations increased separately and in combination.

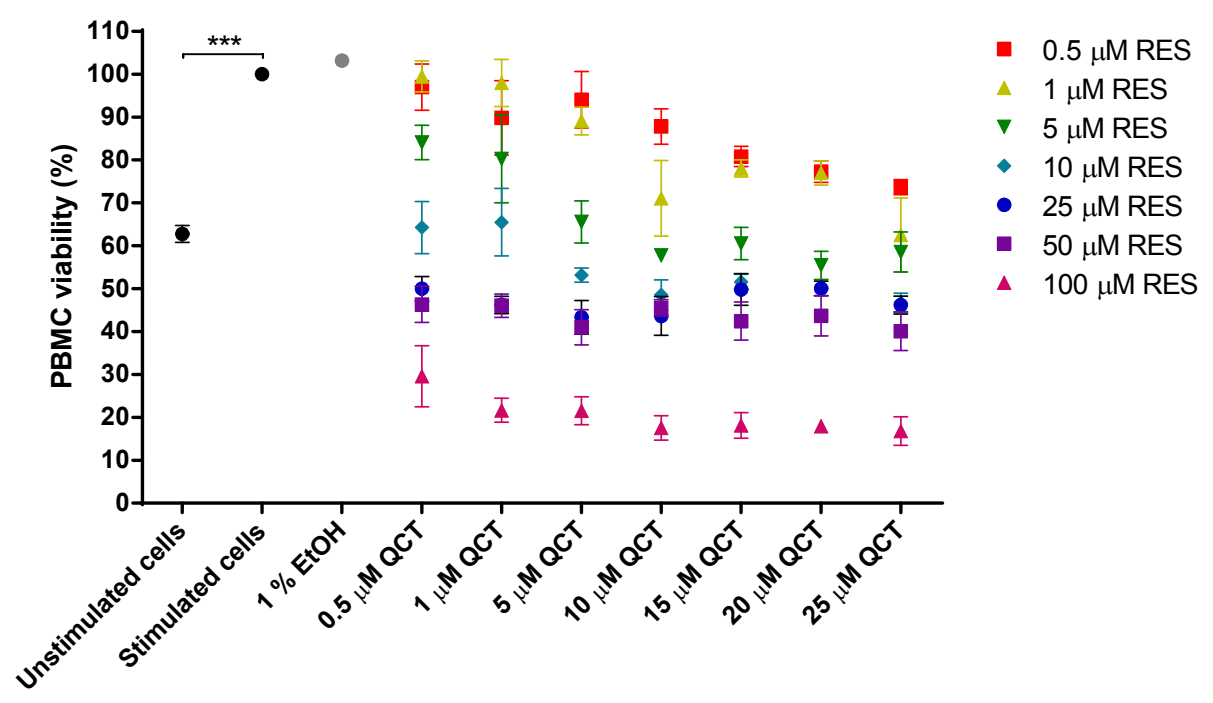

Figure 33. Cytotoxicity of combination of quercetin (QCT) and resveratrol (RES) in peripheral blood mononuclear cells (PBMCs). Data normalised to stimulated cells without treatments. EtOH: ethanol. $\mathrm{N}=3$.

\begin{tabular}{|c|c|c|c|c|c|c|c|c|}
\hline & \multicolumn{8}{|c|}{ QCT $(\mu \mathrm{M})$} \\
\hline \multirow{8}{*}{ 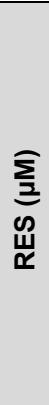 } & & 0.5 & 1 & 5 & 10 & 15 & 20 & 25 \\
\hline & 0.5 & ns & ns & ns & ns & 0.001 & 0.001 & 0.001 \\
\hline & 1 & ns & ns & ns & ns & 0.001 & 0.001 & 0.05 \\
\hline & 5 & ns & ns & 0.05 & 0.001 & 0.001 & 0.001 & 0.05 \\
\hline & 10 & 0.05 & 0.05 & 0.001 & 0.001 & 0.001 & 0.001 & 0.001 \\
\hline & 25 & 0.001 & 0.001 & 0.001 & 0.001 & 0.001 & 0.001 & 0.001 \\
\hline & 50 & 0.001 & 0.001 & 0.001 & 0.001 & 0.001 & 0.001 & 0.001 \\
\hline & 100 & 0.001 & 0.001 & 0.001 & 0.001 & 0.001 & 0.001 & 0.001 \\
\hline
\end{tabular}

Table 7. Statistical differences between quercetin (QCT) + resveratrol (RES) combinations and stimulated cells treated with $1 \%$ ethanol. ns: no significant. 


\section{VI.2.2. Proliferation of PBMCs}

After studying the cytotoxicity effect of polyphenols, it was investigated whether low doses of QCT and RES, and their combination, affect PBMC activation. Cells were activated with anti-CD3/anti-CD28 antibodies and treated with different concentrations of QCT $(0.5,1,5$ and $10 \mu \mathrm{M})$, RES (5 and $10 \mu \mathrm{M})$ and their combinations, as shown in Figure $34(A-B)$. The CFSE proliferation assay (Figure 34A) demonstrated that $5 \mu \mathrm{M}$ RES in combination with 5 and $10 \mu \mathrm{M} Q C T$, and $10 \mu \mathrm{M}$ RES in combination with $0.5,1$, 5 and $10 \mu \mathrm{M}$ QCT inhibited PBMC activation in a dose-dependent manner (all $\mathrm{p}<0.05$ ). In addition, although this did not reach significance, $10 \mu \mathrm{M}$ RES alone tended to decrease PBMC proliferation $(p=0.0826)$, compared to $0.5 \%$ EtOH (Figure 34A). Moreover, there was not a decrease in the percentage of PI negative cells (Figure 34B), which confirmed that these inhibitory effects were not in any case due to a decrease in cell viability after $120 \mathrm{~h}$ of incuabation. 


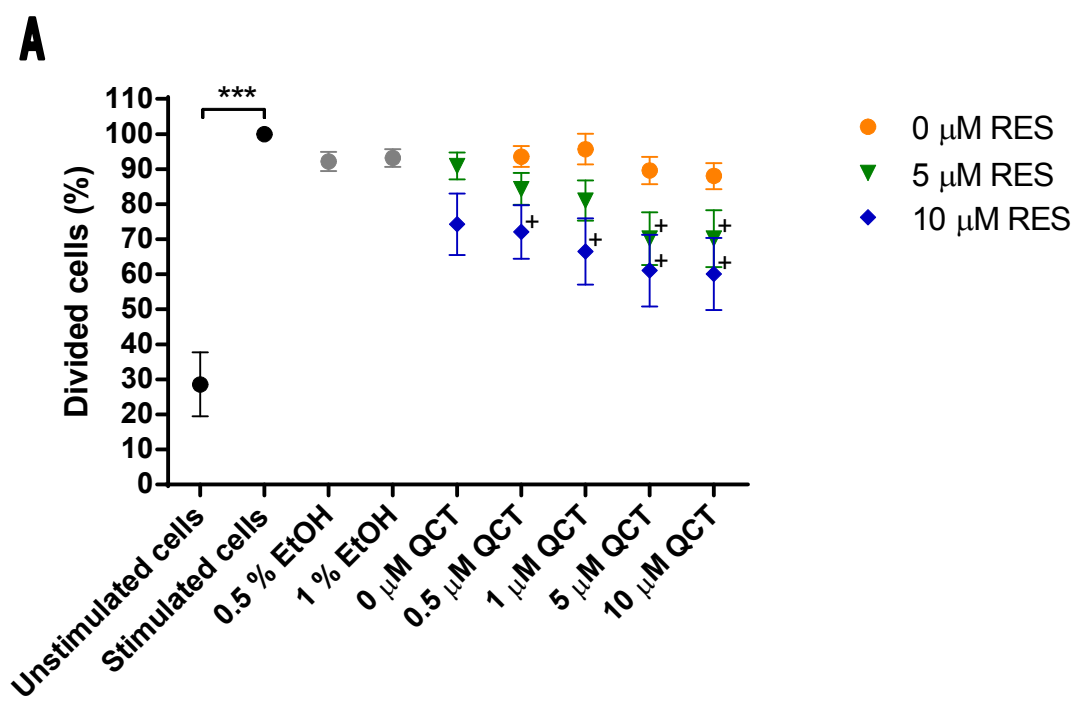

B



Figure 34. Peripheral blood mononuclear cells (PBMCs) treated with quercetin (QCT), resveratrol (RES) and their combination. $A$ : The proliferation of PBMCs evaluated by CFSE labelling assay. B: Percentage of propidium iodide (PI) negative cells. Data are expressed as percentage of cells relative to stimulated cells without treatment. ${ }^{* * *} p<0.001 .{ }^{+} p<0.05$, compared to its vehicle (ethanol: $\mathrm{EtOH}) . \mathrm{N}=3$. 


\section{VI.3. Experimental murine model of dry eye and adoptive transfer model}

This section summarises all of the data obtained from testing topical treatments of polyphenol on the in-vivo model of DED and adoptive transfer model.

\section{VI.3.1. Desiccating stress-induced model of dry eye}

In this study, mice exposed to DS for 10 days (donor mice) were treated topically with $0.01 \%$ QCT, $0.1 \%$ RES and their combination $(0.01 \%$ QCT $+0.1 \%$ RES). CFS, TP, cytokine/chemokine concentration in tears, goblet cell density and $\mathrm{CD} 4^{+} \mathrm{T}$ cell infiltration in conjunctiva were determined.

\section{VI.3.1.1. Corneal fluorescein staining in donor mice}

Visual evaluation of CFS was used as a measure of the effect of DS on corneal epithelial barrier function. Figure 36 shows the CFS score for donor mice. After 9 days, both DS mice and vehicle-treated DS mice showed a significant increase in CFS $(p<0.001)$, compared to control group. There were no differences in staining between DS mice and vehicle-treated DS mice. Topical treatment with $0.01 \%$ QCT $(p<0.001)$ and $0.01 \%$ QCT $+0.1 \%$ RES $(p<0.05)$ showed a significant decrease in CFS, compared to vehicle-treated DS mice. In addition, Figure 37 shows representative CFS images of corneas from each group. Minimal scattered punctate staining, or no staining, was observed on the corneas of control mice after instillation of fluorescein dye (A). Intense punctate fluorescein staining was observed in both DS mice (B) and vehicle-treated DS mice $(C)$. This intense punctate staining decreased when mice were topically treated three times a day with $0.01 \%$ QCT (D), $0.1 \%$ RES (E) and $0.01 \%$ $\mathrm{QCT}+0.1 \% \operatorname{RES}(\mathrm{F})$. 


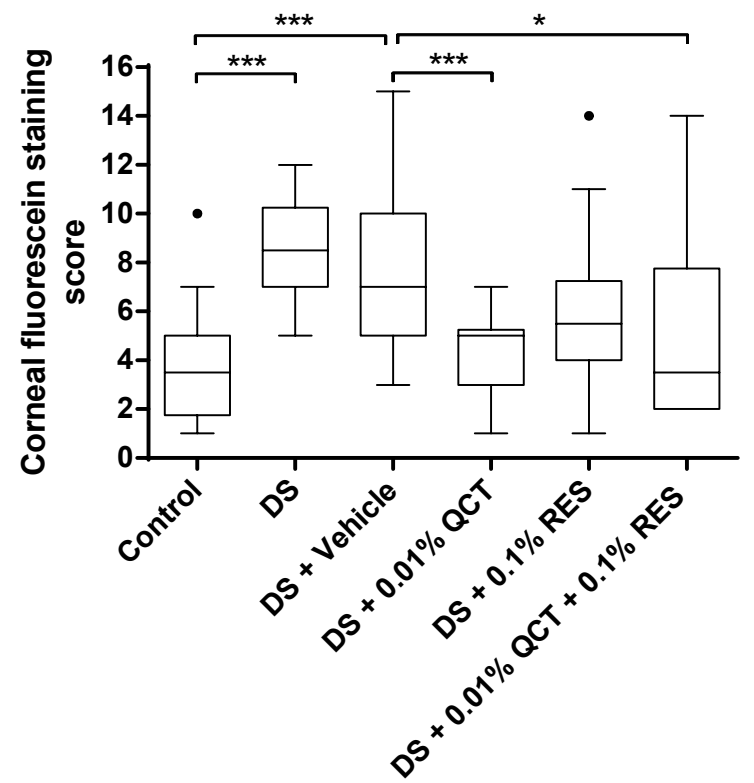

Figure 36. Corneal fluorescein staining in donor mice. Black dots represent outliers. DS: desiccating stress; QCT: quercetin; RES: resveratrol. ${ }^{*} p<0.05 ;{ }^{* * *} p<0.001 . \mathrm{N}=9$.
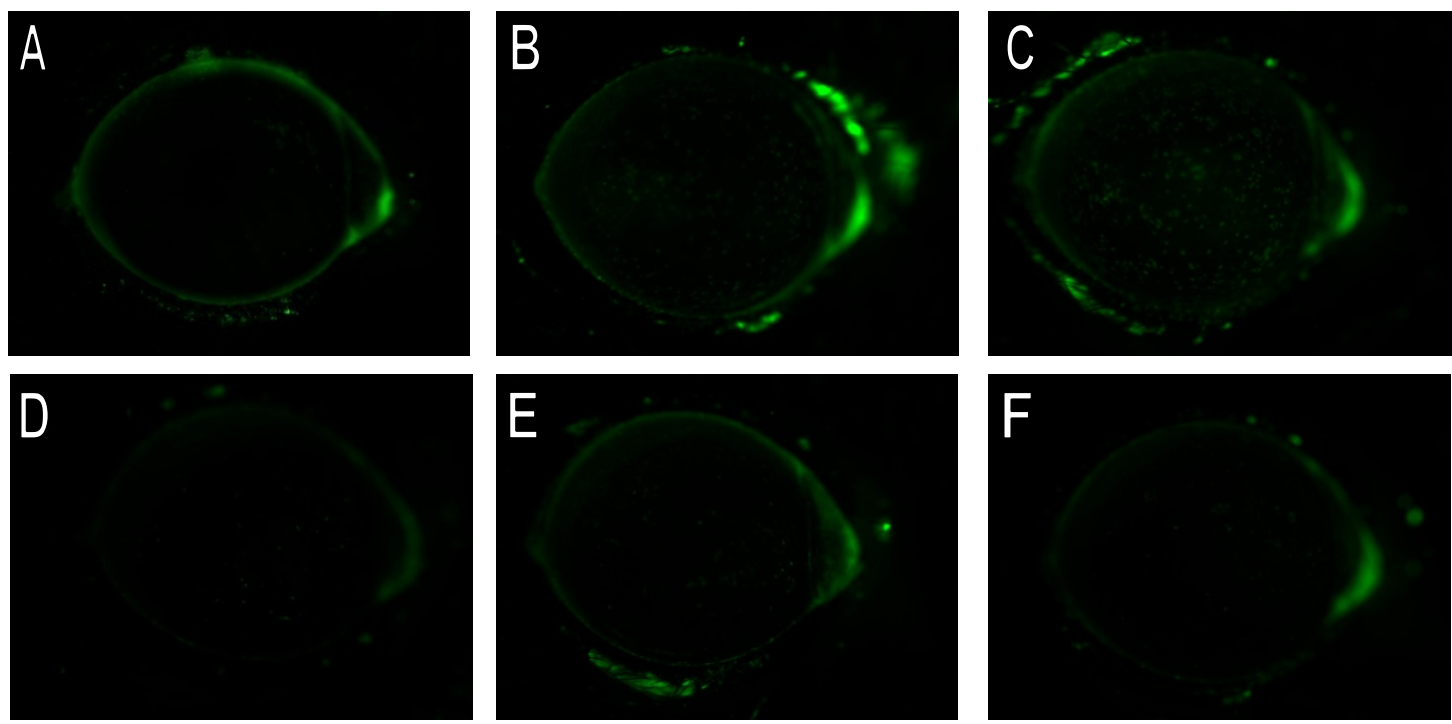

Figure 37. Representative corneal fluorescein staining images of corneas from each group. A: control group; B: desiccating stress (DS) mice; C: vehicle-treated DS mice; D: $0.01 \%$ quercetin (QCT)-treated DS mice; E: $0.1 \%$ resveratrol (RES)-treated DS mice; F: $0.01 \%$ QCT $+0.1 \%$ RES-treated DS mice. $\mathrm{N}=9$.

\section{VI.3.1.2. Aqueous tear production in donor mice}

TP was measured using the phenol red thread test in donor mice, 1 day before DS challenge (baseline) and after 10 days of DS (post-DS). Figure 38 shows the data 
for TP. DS caused a significant decreased in TP $(p<0.001)$, compared to baseline. Neither QCT, nor RES, nor their combination had any effect on TP.

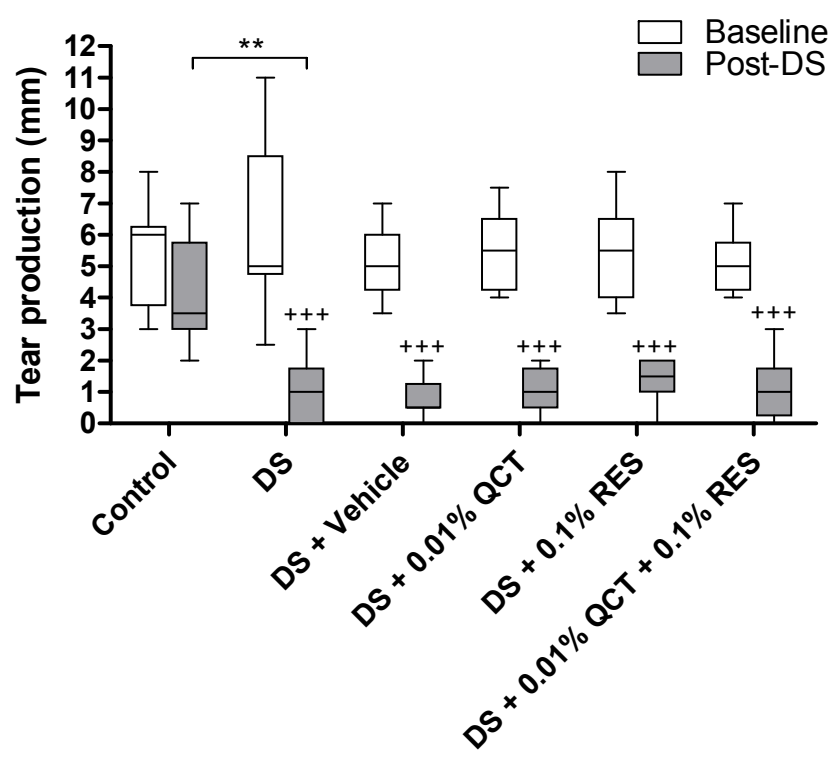

Figure 38. Tear production in donor mice. DS: desiccating stress; QCT: quercetin; RES: resveratrol. ${ }^{* *} p<0.01 .{ }^{+++} p<0.001$, compared to baseline data. $\mathrm{N}=9$.

\section{VI.3.1.3. Cytokine/chemokine concentration in tears of donor mice}

The level of 15 cytokines/chemokines in tears was evaluated using a multiplex bead analysis with $x-M A P^{\circledR}$ technology. Cytokines and chemokines IL1- $\alpha$, IL-2, IL-4, IP10/CXCL10, RANTES/CCL5 and TNF- $\alpha$ were detected in tears at day 6 (Figure 39; the percentage of detected cytokines was $>70 \%$ except for TNF- $\alpha$ : $>50 \%$ ), whilst IL-2, IL4, IP-10/CXCL10 and TNF- $\alpha$ were detected at day 10 (Figure 40; the percentage of detected cytokines was $>60 \%$ for all cytokines). Of the cytokines assessed, DS only significantly increased IL1- $\alpha$ in vehicle-treated DS mice and RANTES/CCL5 in DS mice (both $p<0.05$ ) at day 6 , compared to control mice. Levels of IL1- $\alpha$ decreased when DS mice were treated with $0.01 \%$ QCT $(p<0.05)$, $0.1 \%$ RES $(p<0.01)$, and $0.01 \%$ QCT + $0.1 \%$ RES $(p<0.01)$. At day 6 , although this did not reach significance, there was a trend for decreased IL-4 levels with $0.01 \%$ QCT $(p=0.0625)$, compared to vehicletreated DS mice. There was also a tendency for decreased levels of IP-10/CXCL10 by $0.01 \%$ QCT $(p=0.0850)$, at day 10 (Figure 40$)$. 

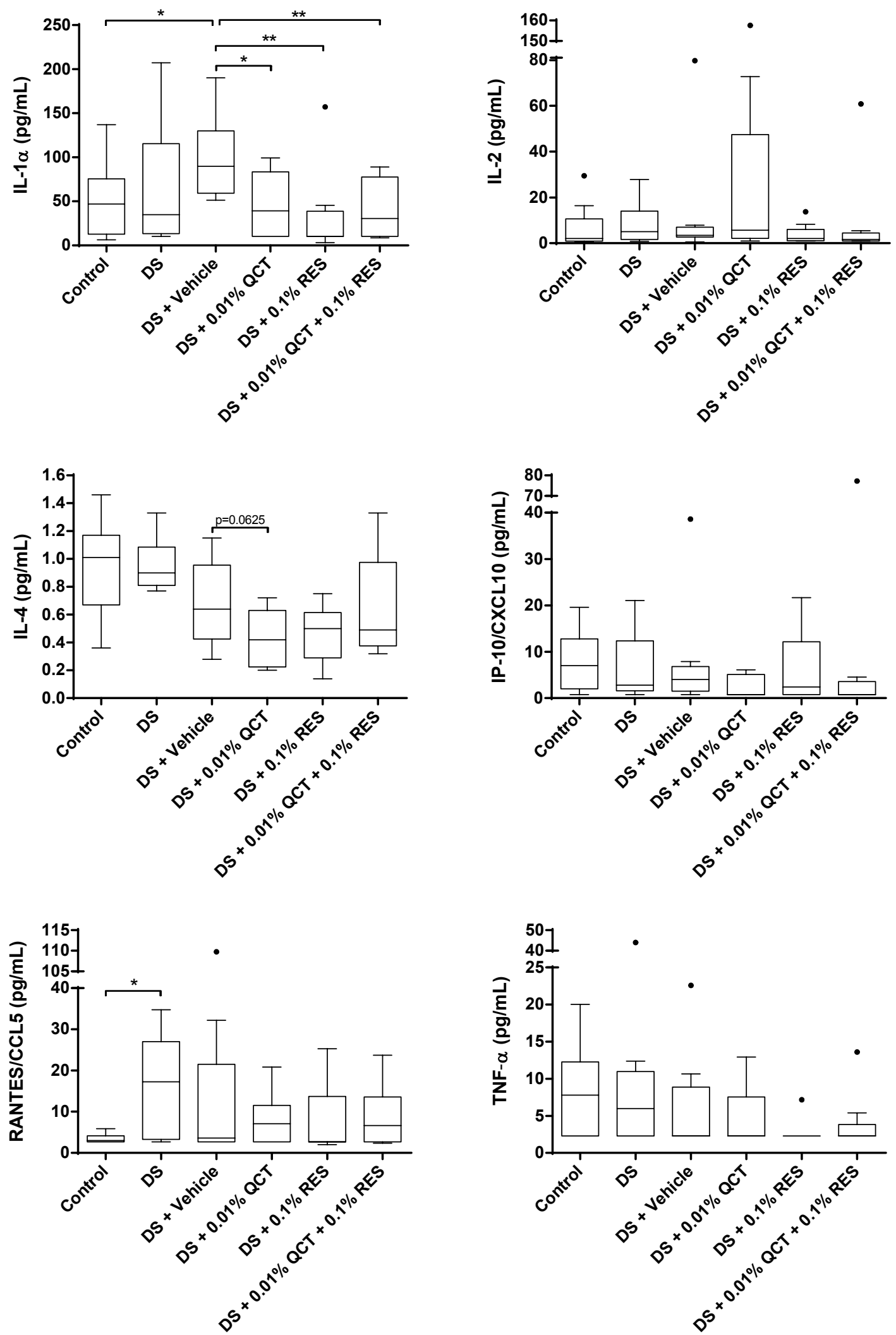

Figure 39. Cytokine/chemokine concentration in donor mouse tears at day 6. Black dots represent outliers. DS: desiccating stress; QCT: quercetin; RES: resveratrol. ${ }^{*} \mathrm{p}<0.05$; ${ }^{* *} \mathrm{p}<0.01$. $\mathrm{N}=9$. 

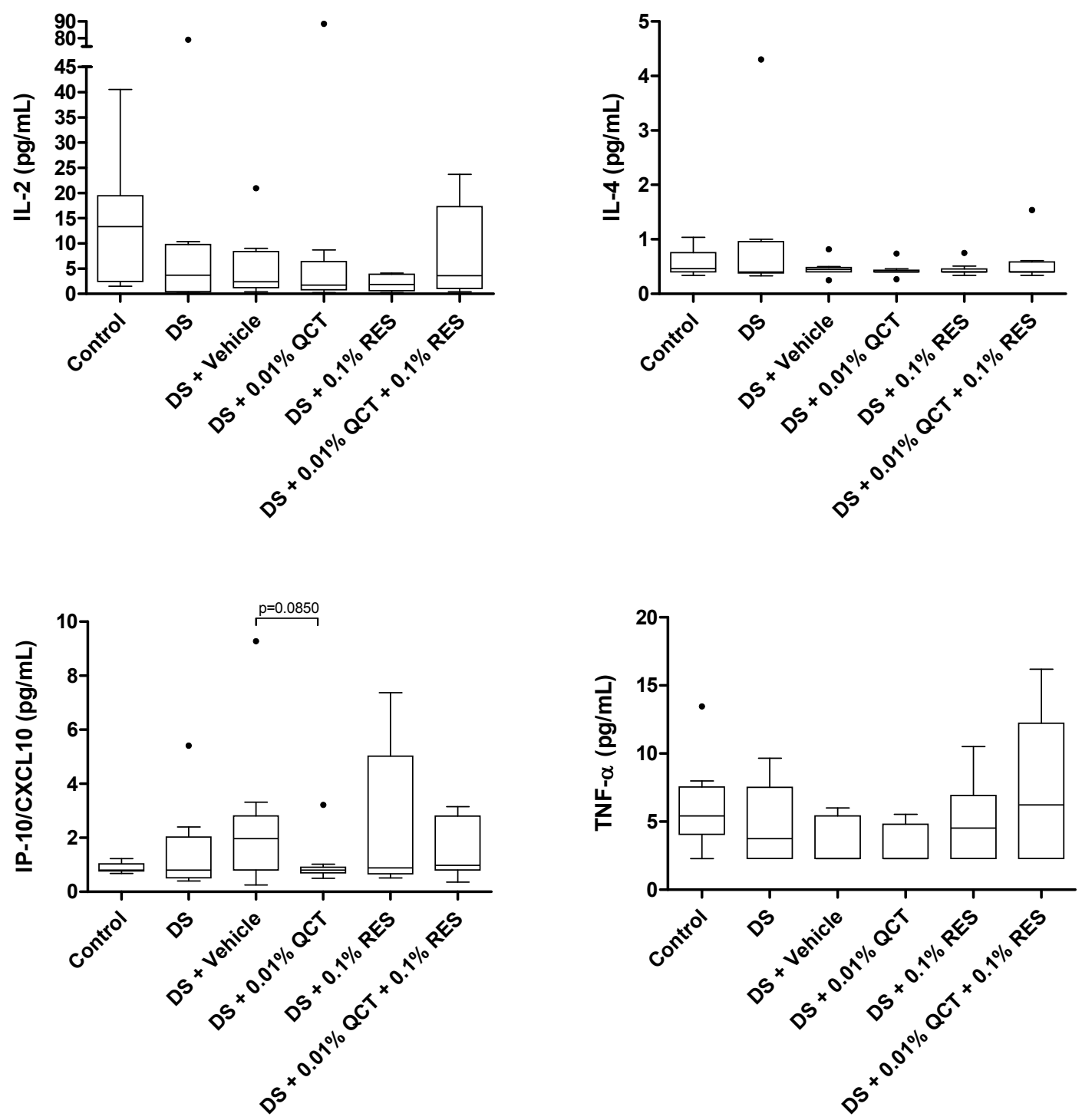

Figure 40. Cytokine/chemokine concentration in donor mouse tears at day 10. Black dots represent outliers. DS: desiccating stress; QCT: quercetin; RES: resveratrol. N=9.

\section{VI.3.1.4. Goblet cell count in donor mice}

Goblet cell numbers in donor conjunctiva were determined using PAS staining. Figure 41 shows the goblet cell count from superior and inferior conjunctivae. Neither DS nor polyphenols altered goblet cell number in donor mice. 


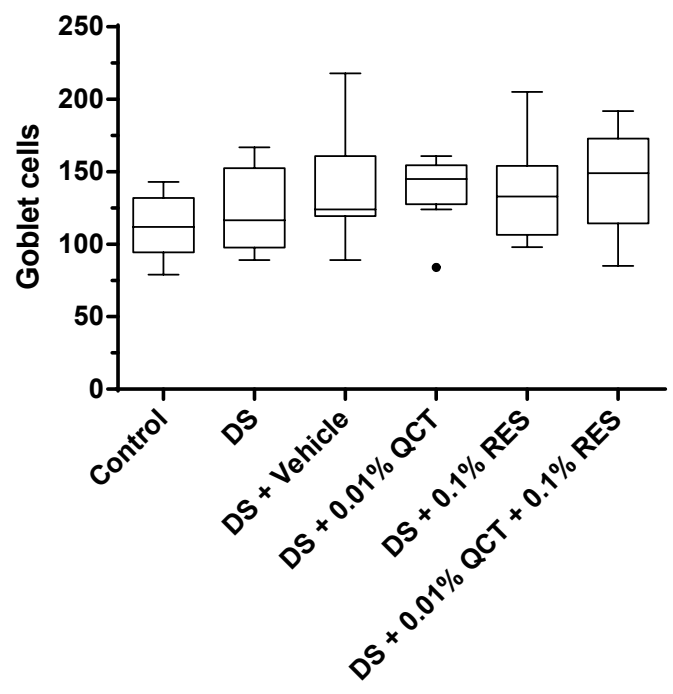

Figure 41. Goblet cell count in donor mouse conjunctiva. Black dots represent outliers. DS: desiccating stress; QCT: quercetin; RES: resveratrol. $\mathrm{N}=9$.

\section{VI.3.1.5. Immunohistochemistry in donor mice}

$\mathrm{CD}^{+} \mathrm{T}$ cell numbers in donor conjunctiva were determined by immunohistochemistry. Figure 42 shows $\mathrm{CD}^{+} \mathrm{T}$ cell count from superior and inferior conjunctivae. Neither DS nor polyphenols altered $\mathrm{CD}^{+} \mathrm{T}$ cell infiltration in donor mouse conjunctiva.

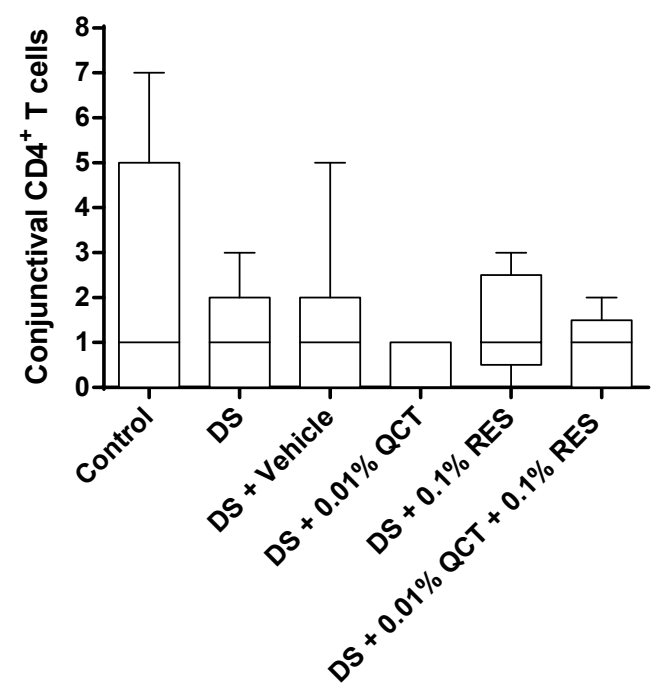

Figure 42. Conjunctival $\mathrm{CD}^{+} \mathrm{T}$ cells in donor mice. DS: desiccating stress; QCT: quercetin; RES: resveratrol. $\mathrm{N}=9$. 


\section{VI.3.2. Adoptive transfer model}

After 10 days of DS, CD4 ${ }^{+} \mathrm{T}$ cells were isolated from donor mice and adoptively transferred to athymic mice (recipient mice). The results from this model are shown in this section.

\section{VI.3.2.1. Aqueous tear production of recipient mice}

TP was measured using the phenol red thread test in donor mice 1 day before transferring $\mathrm{CD}^{+} \mathrm{T}$ cells (baseline), and after $72 \mathrm{~h}$ (post-DS). Figure 43 shows the data for TP in recipient mice. DS tended to decrease TP in recipients of vehicle-treated DS mice ( $p=0.0643$ ), compared to the control. Recipients of $0.01 \%$ QCT $+0.1 \%$ REStreated DS mice significantly increased TP $(p<0.01)$, compared to recipients of vehicletreated DS mice. Neither $0.01 \%$ QCT nor $0.1 \%$ RES had any effect on TP in recipient mice.

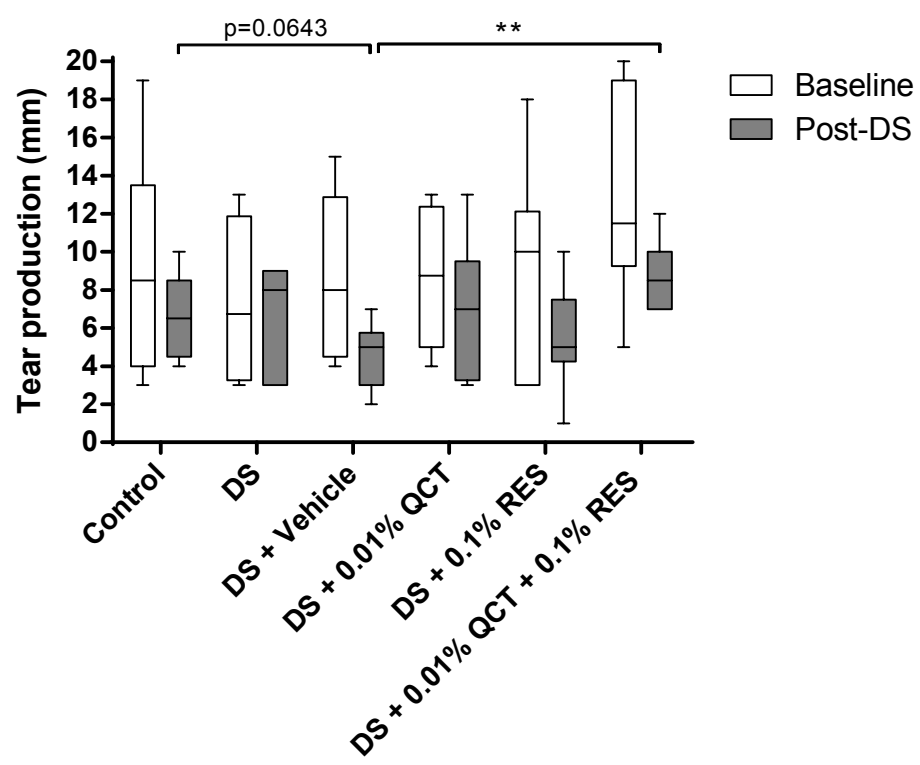

Figure 43. Tear production in recipient mice. DS: desiccating stress; QCT: quercetin; RES: resveratrol. ${ }^{* *} \mathrm{p}<0.01 . \mathrm{N}=8$.

\section{VI.3.2.2. Cytokine/chemokine concentration in tears of recipient mice}

The level of 15 cytokines/chemokines in tears was evaluated as shown in Figure 44. All analysed cytokines/chemokines were detected (percentage of detected cytokines was $>80 \%$ for all cytokines). Cytokine IL-13 tended to decrease in recipient of DS mice ( $p=0.0766)$, compared to recipients of control mice, and IL-17 decreased significantly $(p<0.05)$ in recipients of vehicle-treated DS mice, compared to recipients of control mice. Neither $0.01 \%$ QCT, nor $0.1 \%$ RES, nor $0.01 \%$ QCT + $0.1 \%$ RES had any effect on cytokine/chemokine levels in recipient mice. 

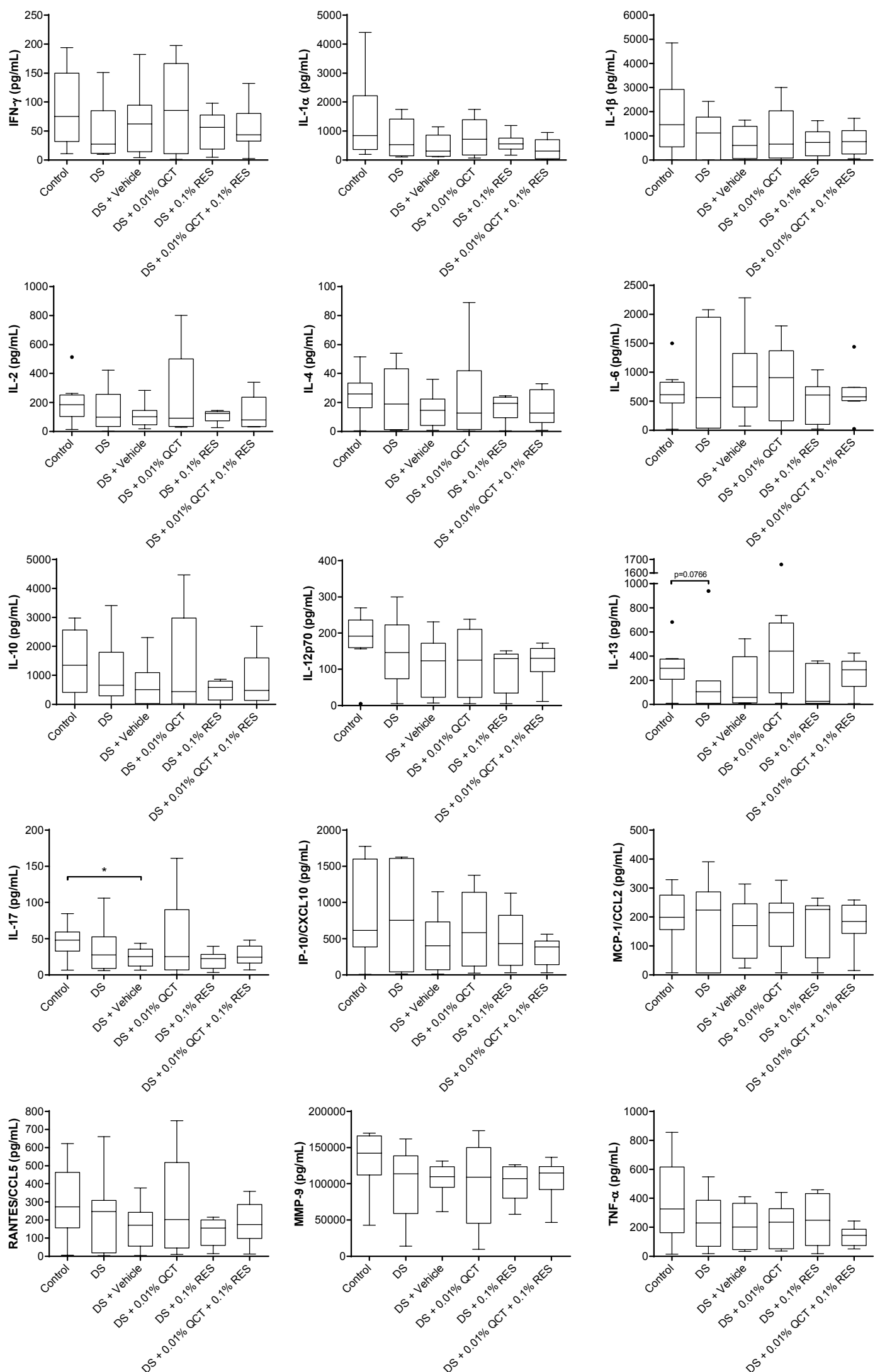

Figure 44. Cytokine/chemokine concentration in recipient mouse tears at day 3. Black dots represent outliers. DS: desiccating stress; QCT: quercetin; RES: resveratrol. * $p<0.05$. $N=8$. 


\section{VI.3.2.3. Goblet cell count in recipient mice}

Goblet cell numbers in recipient conjunctiva were determined using PAS stain. Figure 45 shows goblet cell count from superior and inferior conjunctivae. Neither DS nor polyphenols altered goblet cell numbers in recipient mice.

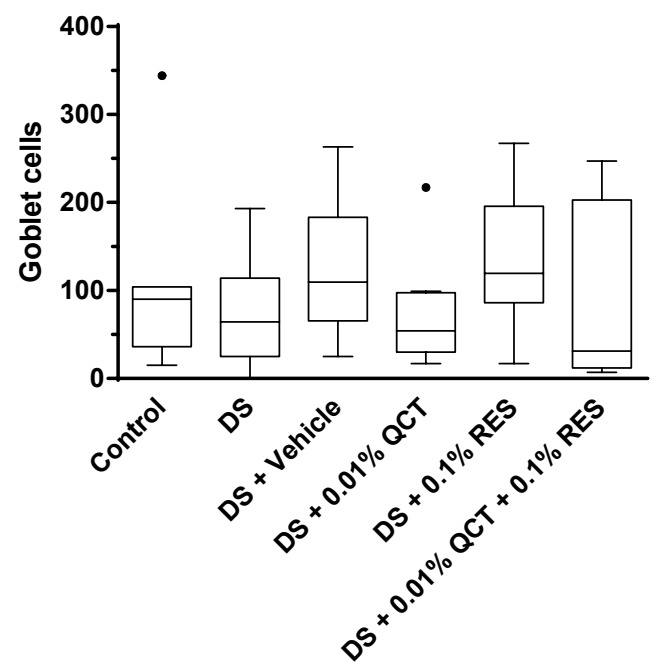

Figure 45. Goblet cell count in recipient mouse conjunctiva. Black dots represent outliers. DS: desiccating stress; QCT: quercetin; RES: resveratrol. $\mathrm{N}=8$.

\section{VI.3.2.4. Immunohistochemistry in recipient mice}

$\mathrm{CD}^{+} \mathrm{T}$ cell number was determined by immunohistochemistry. Figure $46 \mathrm{~A}$ shows representative immunostaining images of $\mathrm{CD}^{+} \mathrm{T}$ cells from each group and Figure 46B shows $\mathrm{CD}^{+} \mathrm{T}$ cell infiltration in the superior and inferior recipient mouse conjunctiva. DS produced a tendency to increased $\mathrm{CD} 4^{+} \mathrm{T}$ cells in conjunctiva of recipients of DS mice $(p=0.0715)$. No significant differences were found in recipients of vehicle-treated DS mice and recipients of DS mice. Regarding topical treatments with polyphenols, $0.1 \%$ RES decreased $\mathrm{CD}^{+} \mathrm{T}$ cell infiltration in conjunctiva of recipients of RES-treated DS mice $(p<0.05)$. QCT $(0.01 \%)$ showed a tendency for decreased $\mathrm{CD}^{+} \mathrm{T}$ cell infiltration $(\mathrm{p}=0.0743)$, compared to recipients of vehicle-treated DS mice. 
A
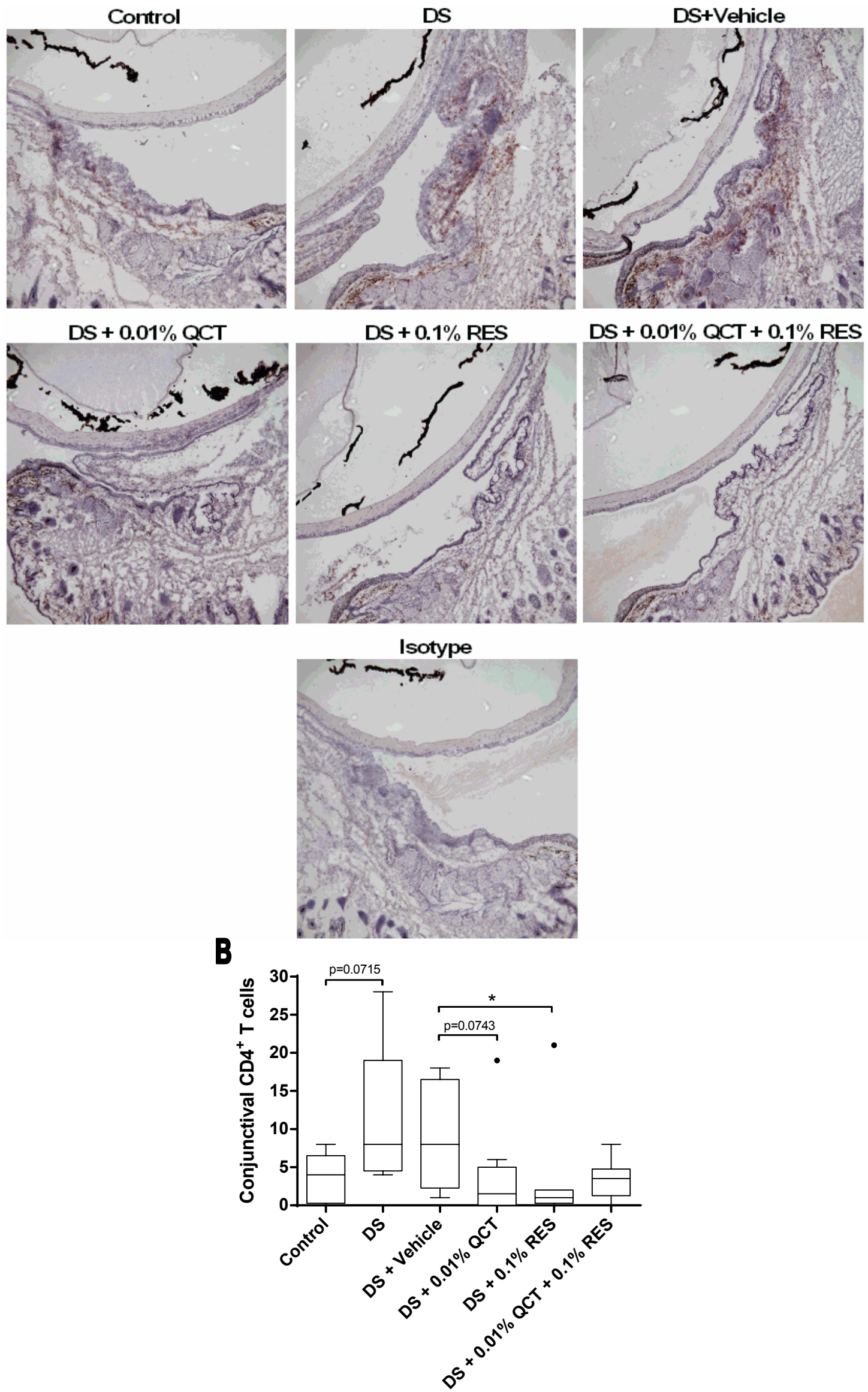

Figure 46. $\mathrm{CD}^{+} \mathrm{T}$ cell infiltration in conjunctiva of recipient mouse. $\mathrm{A}$ : Representative immunostaining images from each group. Original magnification 10X. B: CD4 ${ }^{+} \mathrm{T}$ cell infiltration in conjunctiva. Isotype were used as a negative control. Black dots represent outliers. DS: desiccating stress; QCT: quercetin; RES: resveratrol. * $\mathrm{p}<0.05$. $\mathrm{N}=8$. 


\section{VI.4. In-vitro model of conjunctival mast cell response}

This section summarises all of the data obtained from testing the anti-allergic effect of polyphenols on CBMCs.

\section{VI.4.1. Cytotoxicity of polyphenols}

The cytotoxicity of QCT, RES and QCT+RES in CBMCs stimulated with $\lg \mathrm{E} / \mathrm{anti}-\mathrm{IgE}$ after $24 \mathrm{~h}$ exposure was tested using PI staining and flow cytometry.

\section{VI.4.1.1. $\underline{\text { Cytotoxicity of QCT }}$}

Figure 47 shows the cytotoxicity of QCT in CBMCs. Seven concentrations of QCT were tested: $0.5,1,5,10,15,20$ and $25 \mu \mathrm{M}$. None of the QCT concentrations used were toxic for CBMCs after $24 \mathrm{~h}$ of culture.

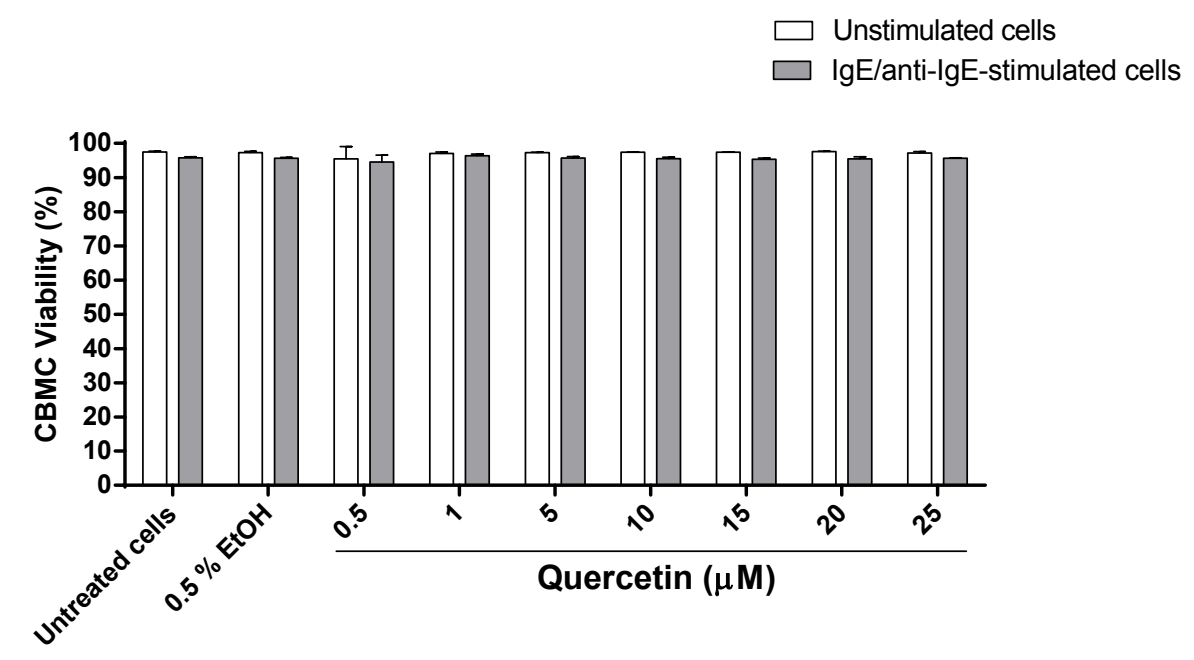

Figure 47. Cytotoxicity of quercetin (QCT) in cord blood mast cells (CBMCs). EtOH: ethanol. $\mathrm{N}=1$.

\section{VI.4.1.2. Cytotoxicity of RES}

Figure 48 shows the cytotoxicity of RES in CBMCs. Seven concentrations of RES were tested: $0.5,1,5,10,25,50$ and $100 \mu \mathrm{M}$. None of the RES concentrations used were toxic for CBMCs. 


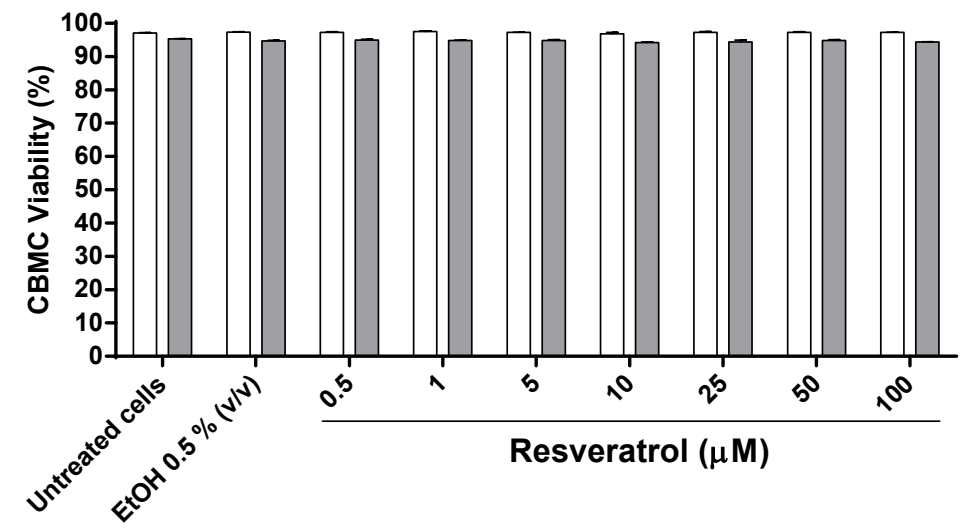

Figure 48. Cytotoxicity of resveratrol (RES) in cord blood mast cells (CBMCs). Ethanol: $\mathrm{EtOH} . \mathrm{N}=1$.

\section{VI.4.1.3. $\underline{\text { Cytotoxicity of combinations of QCT and RES }}$}

Figure 49 shows the cytotoxicity of combinations of QCT and RES in CBMCs. Seven concentrations each of QCT $(0.5,1,5,10,15,20$ and $25 \mu \mathrm{M})$ and RES $(0.5,1$, $5,10,25,50$ and $100 \mu \mathrm{M}$ ) were tested alone and in combination with each other. None of the combinations of QCT+RES affected CBMC viability.

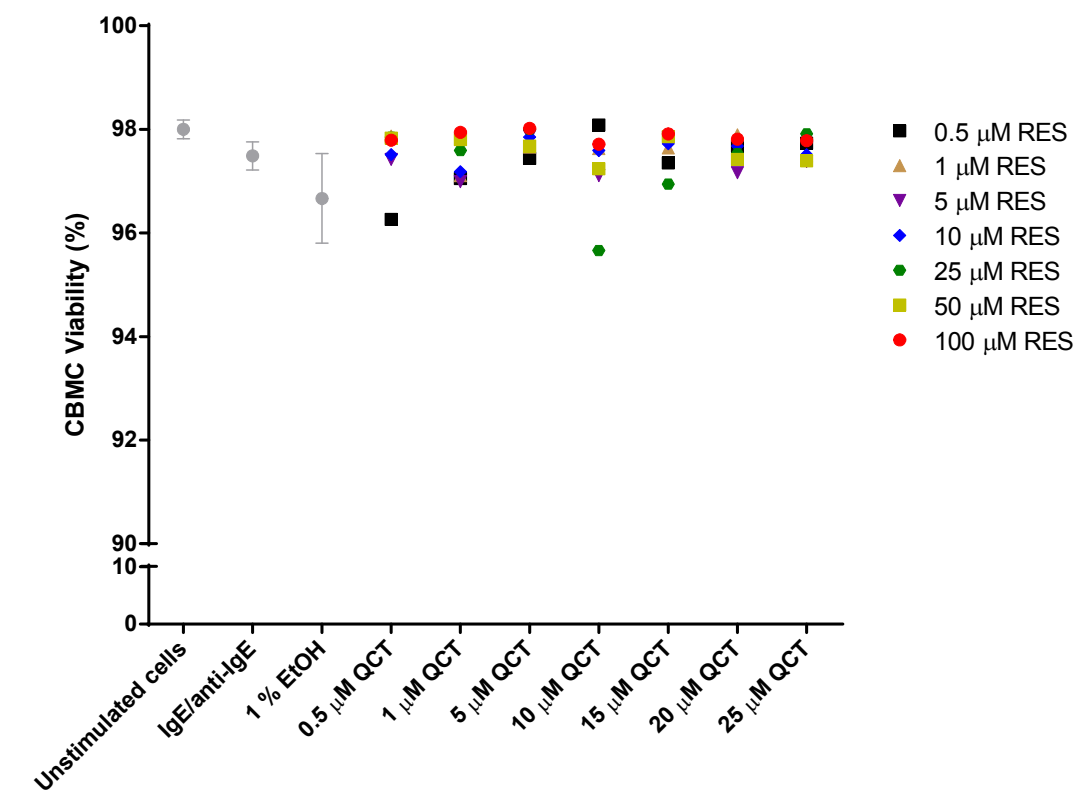

Figure 49. Cytotoxicity of combination of quercetin (QCT) and resveratrol (RES) in cord blood mast cells (CBMCs). EtOH: ethanol. $\mathrm{N}=1$. 


\section{VI.4.2. Histamine release}

Dose-response curves for studying the effect of QCT, RES and their combinations on histamine release were firstly obtained from two different experiments performed in single samples.

\section{VI.4.2.1. Effect of QCT on histamine release}

Figure 50 shows the effect of different concentrations of QCT on histamine release by CBMCs. One hour following IgE/anti-lgE stimulation, histamine secretion was increased $(p<0.001)$. This increased secretion of histamine was significantly reduced by vehicle $(0.5 \% \mathrm{EtOH})(p<0.001)$. Histamine secretion by stimulated CBMCs treated with vehicle was only significantly decreased by $0.5 \mu \mathrm{M} Q \mathrm{QT} \quad(p<0.01)$. However, there was a tendency for decreased histamine secretion by QCT at concentrations of $1 \mu \mathrm{M}$ ( $p=0.0556), 10 \mu \mathrm{M}$ ( $p=0.0504), 15 \mu \mathrm{M}(\mathrm{p}=0.0509), 20 \mu \mathrm{M}$ $(p=0.0556)$ and $25 \mu \mathrm{M}(p=0.0513)$.

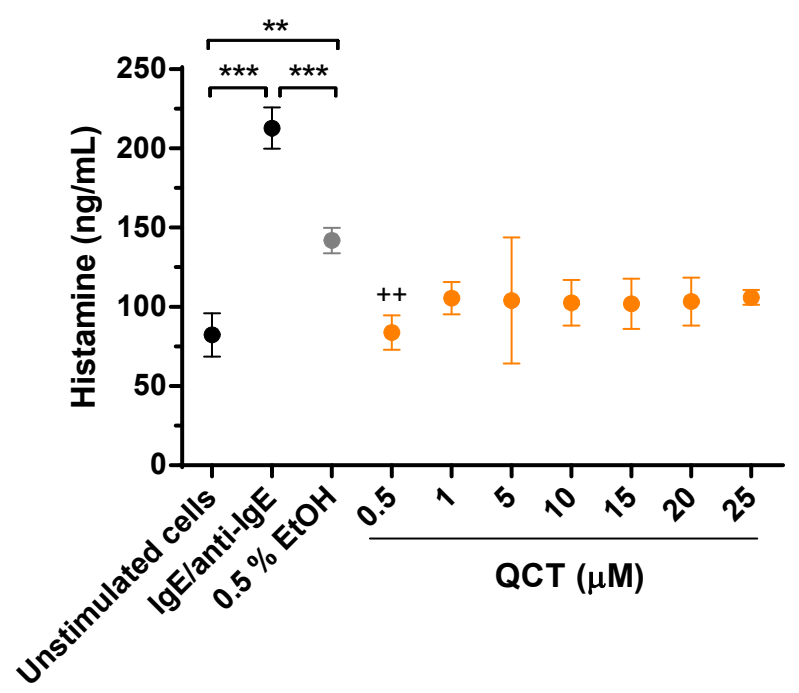

Figure 50. Effect of quercetin (QCT) on histamine secretion. ${ }^{* *} p<0.01$, ${ }^{* * *} p<0.001 ;{ }^{++} p<0.01$, compared to stimulated cells treated with vehicle: ethanol (EtOH). $\mathrm{N}=2$ (single samples).

\section{VI.4.2.2. Effect of RES on histamine release}

Figure 51 shows the effect of different RES concentrations on histamine release by CBMCs. RES did not have any significant effect on histamine release, except for $5 \mu \mathrm{M}$ RES, which showed a trend for decreased histamine secretion $(p=0.0804)$. 


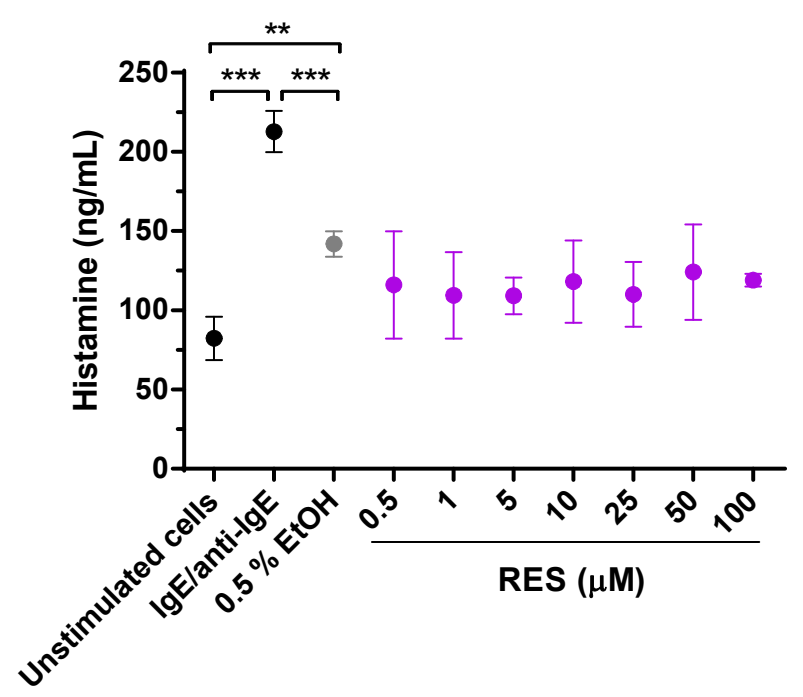

Figure 51. Effect of resveratrol (RES) on histamine secretion. EtOH: ethanol. ${ }^{* *} p<0.01,{ }^{* * *} p<0.001 . N=2$ (single samples).

\section{VI.4.2.3. Effect of QCT+RES combination on histamine release}

Figure 52 ( $A$ \& B) shows the effect of different QCT+RES combinations on histamine release by CBMCs. For better comprehension, Figures $52 \mathrm{~A}$ and $\mathrm{B}$ represent all data with and without plotting SEM, respectively. Table 8 shows the significant differences for each combination of QCT+RES, compared to stimulated cells treated with vehicle, $1 \% \mathrm{EtOH}$. Histamine secretion by stimulated CBMCs treated with vehicle was significantly decreased by $5 \mu \mathrm{M}$ QCT $+25 \mu \mathrm{M}$ RES, $10 \mu \mathrm{M}$ QCT $+25 \mu \mathrm{M}$ RES, 0.5 $\mu \mathrm{M} Q C T+50 \mu \mathrm{M}$ RES and $10 \mu \mathrm{M} \mathrm{QCT}+100 \mu \mathrm{M}$ RES (all $p<0.05$ ). 
A

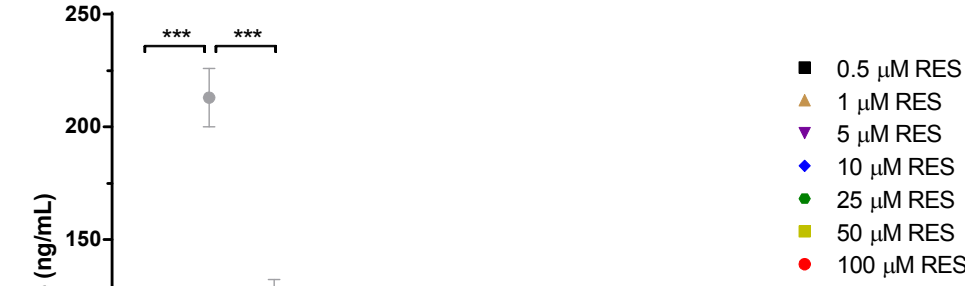

B

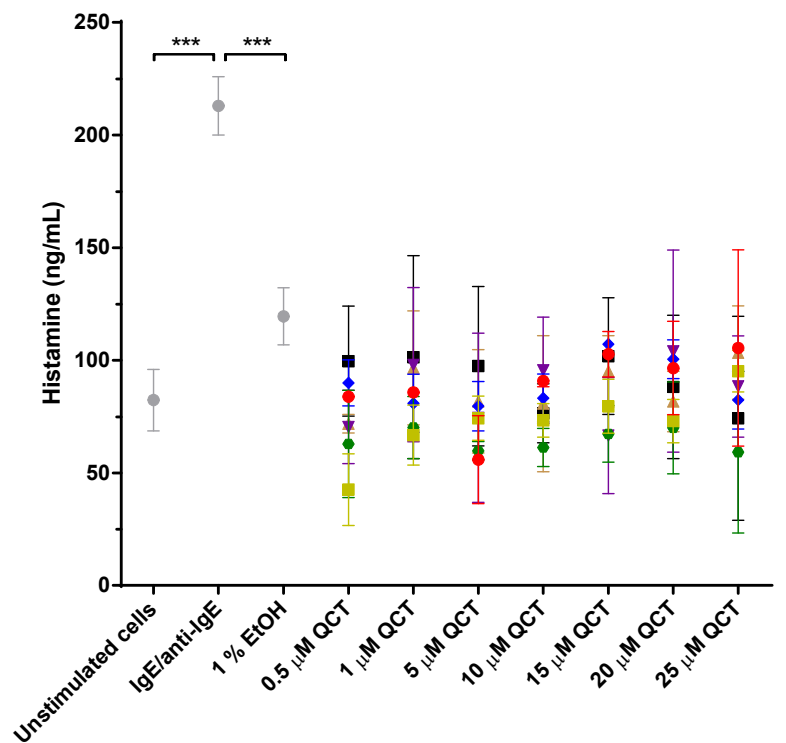

- $0.5 \mu \mathrm{M}$ RES

$1 \mu \mathrm{M}$ RES

$\checkmark 5 \mu \mathrm{M} R E S$

- $10 \mu \mathrm{M}$ RES

- $25 \mu \mathrm{M}$ RES

- $50 \mu \mathrm{M}$ RES

- $100 \mu \mathrm{M}$ RES

Figure 52. Effect of combination of quercetin (QCT) and resveratrol (RES) on histamine secretion. EtOH: ethanol. $\mathrm{N}=2$ (single samples).

\begin{tabular}{|c|c|c|c|c|c|c|c|c|}
\hline & \multicolumn{8}{|c|}{ QCT $(\mu \mathrm{M})$} \\
\hline \multirow{8}{*}{ 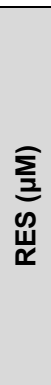 } & & 0.5 & 1 & 5 & 10 & 15 & 20 & 25 \\
\hline & 0.5 & ns & ns & $\mathrm{ns}$ & $\mathrm{ns}$ & $\mathrm{ns}$ & ns & ns \\
\hline & 1 & 0.0861 & ns & ns & ns & ns & ns & ns \\
\hline & 5 & ns & ns & ns & ns & 0.0893 & ns & ns \\
\hline & 10 & ns & ns & ns & ns & $\mathrm{ns}$ & ns & ns \\
\hline & 25 & ns & 0.0859 & 0.05 & 0.05 & 0.0719 & ns & 0.0802 \\
\hline & 50 & 0.05 & 0.0706 & ns & ns & ns & ns & ns \\
\hline & 100 & ns & ns & 0.05 & $\mathrm{~ns}$ & ns & ns & ns \\
\hline
\end{tabular}

Table 8. Statistical differences between quercetin(QCT) + resveratrol(RES) combinations and stimulated cells treated with vehicle ( $1 \%$ ethanol). Ns: no significant. 
After dose-response curves were obtained from two different experiments carried out in single samples, a combination of QCT+RES (10 $\mu \mathrm{M} Q C T+25 \mu \mathrm{M}$ RES), which produced a significant decrease in histamine secretion, was chosen in order to study the effect of a polyphenol combination (in an experiment performed in triplicate). Figure 53 shows the effect of $10 \mu \mathrm{M}$ QCT, $25 \mu \mathrm{M}$ RES and $10 \mu \mathrm{M}$ QCT + $25 \mu \mathrm{M}$ RES on histamine release by CBMCs. Stimulated cells treated with $0.5 \% \mathrm{EtOH}$ showed a significant decrease of histamine release compared to control cells $(p<0.001)$. Moreover, $10 \mu \mathrm{M}$ QCT and $25 \mu \mathrm{M}$ RES significantly decreased histamine release of stimulated CBMCs (both $\mathrm{p}<0.01$ ). The mix of both compounds did not have any effect on histamine release in stimulated cells.

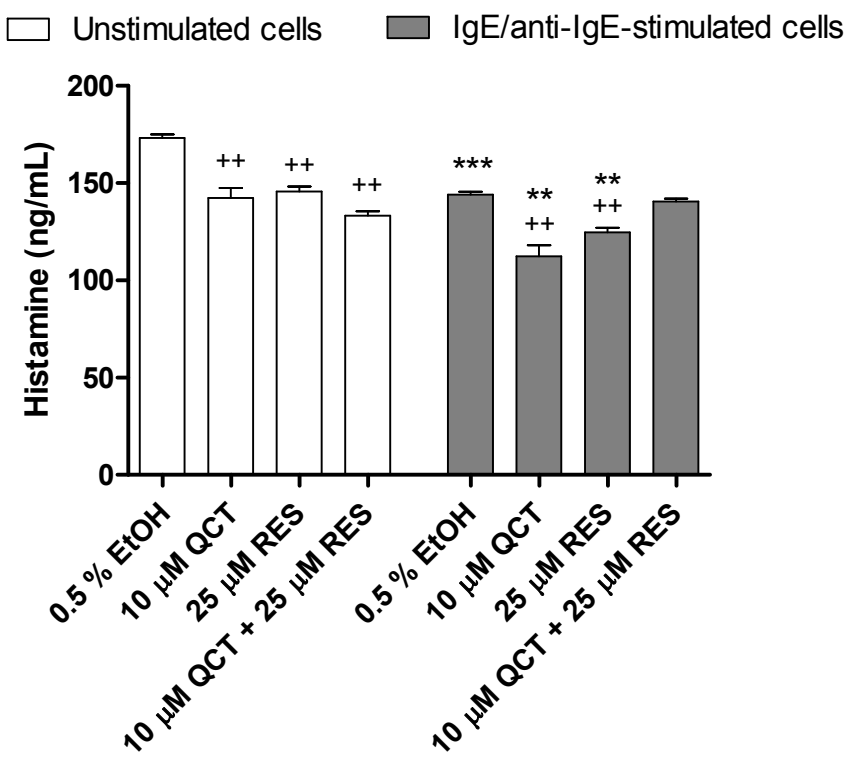

Figure 53. Effect of quercetin (QCT), resveratrol (RES) and their combination on histamine secretion. ${ }^{* *} p<0.01,{ }^{* * *} p<0.001$, compared to unstimulated cells; ${ }^{++} p<0.01$, compared to cells treated with ethanol (EtOH). $\mathrm{N}=1$ (triplicate).

\section{VI.4.3. Cytokine/chemokine secretion}

After determining the effect of different concentrations of QCT, RES and their combinations on histamine release, the effect of polyphenols on cytokine release after $24 \mathrm{~h}$ was studied. For this purpose, secreted cytokine dose-response curves were first obtained for QCT $(0.5,1,5,10,15,20$ and $25 \mu \mathrm{M})$ and $\operatorname{RES}(0.5,1,5,10,25,50$ and $100 \mu \mathrm{M}$ ) in CBMCs stimulated by $\operatorname{lgE} / a n t i-\lg E$ (from two different experiments performed in single samples). After that, the two concentrations of polyphenols tested on histamine release (10 $\mu \mathrm{M}$ QCT and $25 \mu \mathrm{M}$ RES) and both compounds alone were 
also tested in order to determine the effect of both compounds together (one experiment performed in triplicate).

For a better comprehension of all of the data presented herein, results are subdivided into nine sections, according to the studied cytokines/chemokines: IL-1 $\beta$, IL4, IL-5, IL-8/CXCL8, IL-9, IL-10, IL-13, TNF- $\alpha$ and IFN-y.

\section{VI.4.3.1. IL-1 $\underline{\mathrm{L} \text { secretion }}$}

IL-1 $\beta$ secretion was not detected in any sample.

\section{VI.4.3.2. IL-4 secretion}

Figure $54(\mathrm{~A}-\mathrm{C})$ shows the effect of QCT, RES and their combination on IL-4 secretion. Stimulation with IgE/anti-IgE did not change IL-4 basal secretion by CBMCs. Neither QCT, nor RES, nor QCT+RES had any effect on IL-4 secretion. 
A



B


Figure 54. Effect of quercetin (QCT) (A), resveratrol (RES) (B) and QCT+RES (C) on IL-4 secretion. EtOH: ethanol. $\mathrm{N}=2$ for $\mathrm{A}$ and $\mathrm{B}$ (single samples); and $\mathrm{N}=1$ for $\mathrm{C}$ (triplicate). 


\section{VI.4.3.3. IL-5 secretion}

Figure 55 (A-C) shows the effect of QCT, RES and their combination on IL-5 secretion. QCT increased IL-5 levels at 1 and $10 \mu \mathrm{M}(\mathrm{p}<0.05$; Figure 55A). On the other hand RES decreased IL-5 secretion at $25 \mu \mathrm{M}$ (Figure 55B). IL-5 secretion was only stimulated by IgE/anti-IgE in CBMCs when compounds were tested together (Figure 55C). QCT+RES had no effect on IL-5 secretion (Figure 55C). 
A

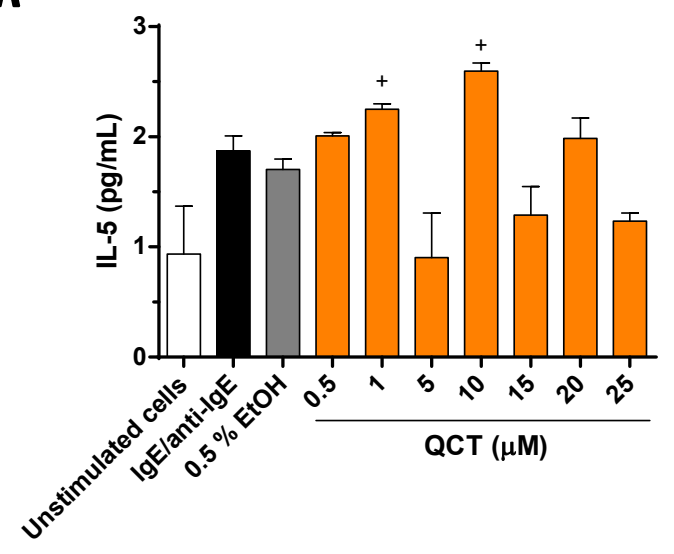

B
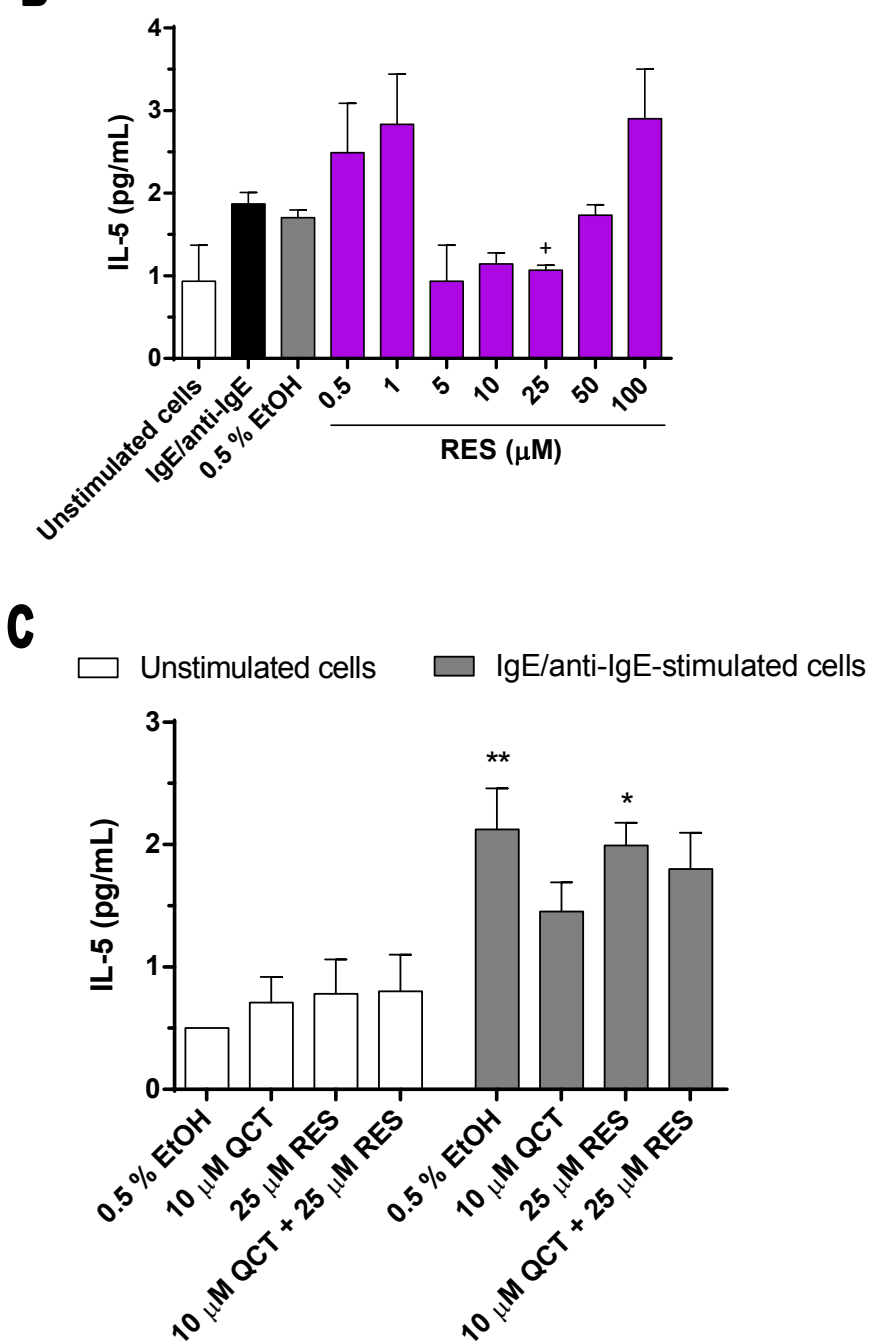

Figure 55. Effect of quercetin (QCT) (A), resveratrol (RES) (B) and QCT+RES (C) on IL-5 secretion. ${ }^{*} \mathrm{p}<0.05,{ }^{*} \mathrm{p}<0.01$, compared to unstimulated cells; ${ }^{+} p<0.05$, compared to stimulated cells treated with ethanol (EtOH). N=2 for A and B (single samples); and $\mathrm{N}=1$ for $\mathrm{C}$ (triplicate). 


\section{VI.4.3.4. IL-8/CXCL8 secretion}

Figure $56(A-C)$ shows the effect of QCT, RES and their combination on IL-8 secretion. Stimulation of CBMCs with IgE/anti-lgE induced a significant increase of IL8/CXCL8, compared to control cells ( $p<0.05$; Figures $56 \mathrm{~A}$ and $B$ ). When cells were treated with polyphenols, this stimulated secretion of IL-8/CXCL8 was decreased at 10 , 15 and $20 \mu \mathrm{M}$ QCT (all $p<0.01$; Figure 56A), whilst RES decreased IL-8/CXCL8 secretion at $10,25,50$ and $100 \mu \mathrm{M}(\mathrm{p}<0.05, \mathrm{p}<0.01, p<0.01$ and $\mathrm{p}<0.01$, respectively; Figure 56B), compared to IgE/anti-lgE-stimulated cells treated with EtOH. The mix of both compounds did not have a significant effect on IL-8/CXCL8 production by CBMCs but its level was lower that stimulated cells treated with vehicle. 
A

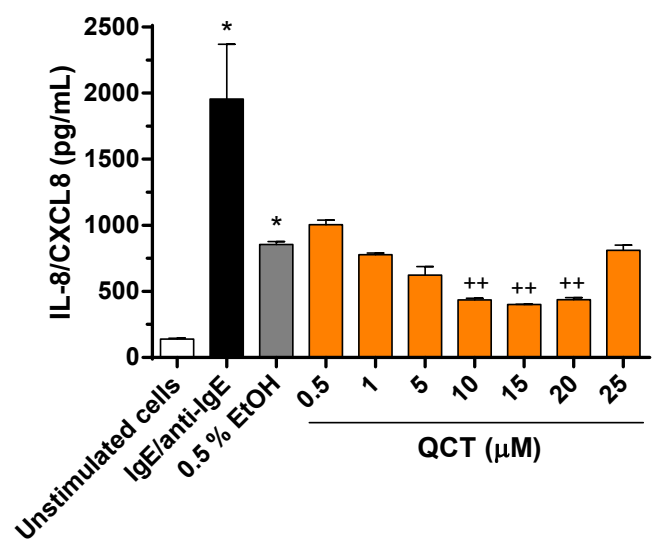

B

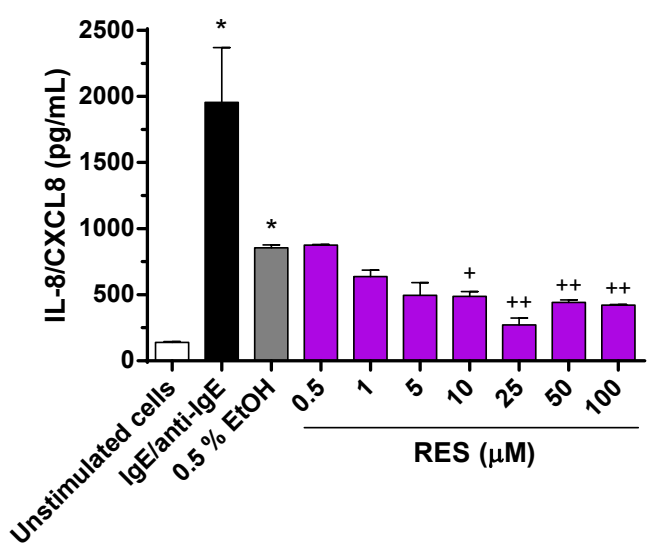

C

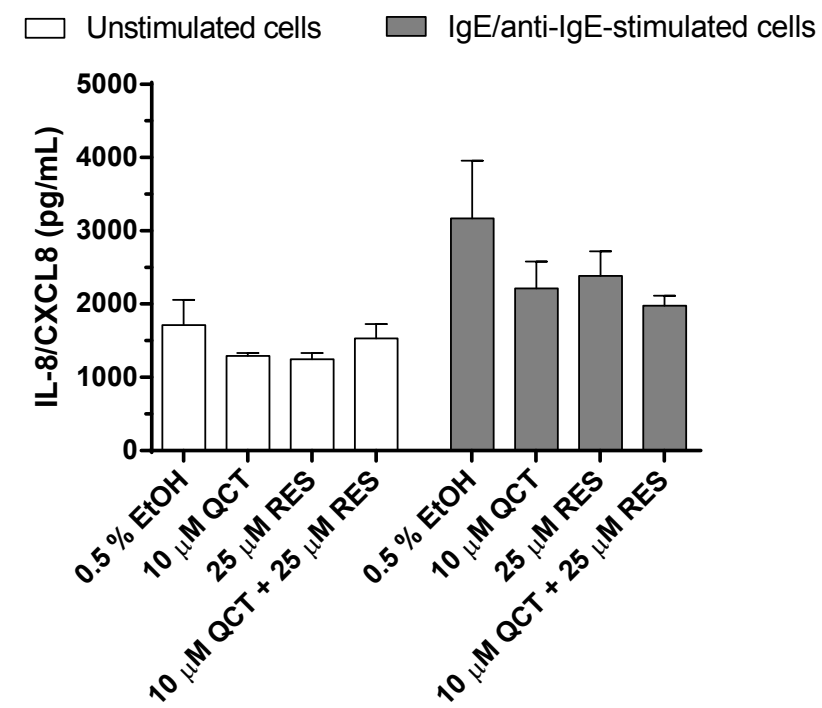

Figure 56. Effect of quercetin (QCT) (A), resveratrol (RES) (B) and QCT+RES (C) on IL-8/CXCL8 secretion. * $p<0.05$, compared to unstimulated cells; ${ }^{+} p<0.05,{ }^{++} p<0.01$, compared to stimulated cells treated with ethanol $(\mathrm{EtOH}) . \mathrm{N}=2$ for $\mathrm{A}$ and $\mathrm{B}$ (single samples); and $\mathrm{N}=1$ for $\mathrm{C}$ (triplicate). 
VI.4.3.5. IL-9 secretion

IL-9 secretion was not detected in any sample.

VI.4.3.6. $\underline{\mathrm{LL}-10 \text { secretion }}$

Figure 57 (A-C) shows the effect of QCT, RES and their combination on IL-10 secretion. IgE/anti-IgE stimulation did not induce IL-10 secretion by CBMCs. Neither QCT, nor RES, nor their combination had any significant effect on IL-10 production. 
A



B

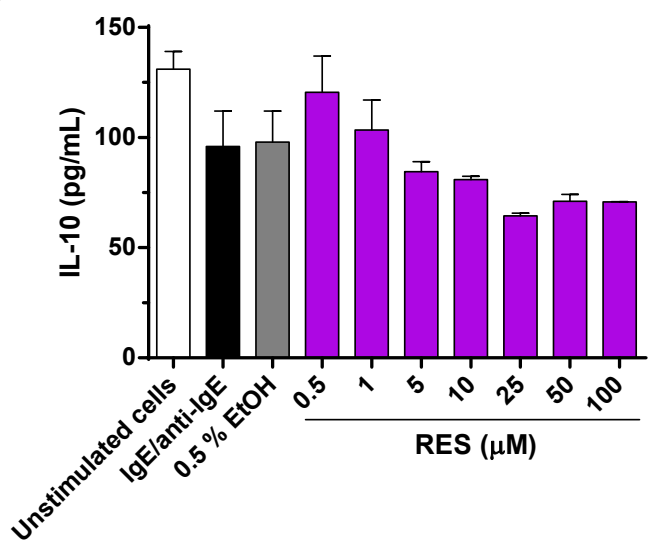

C

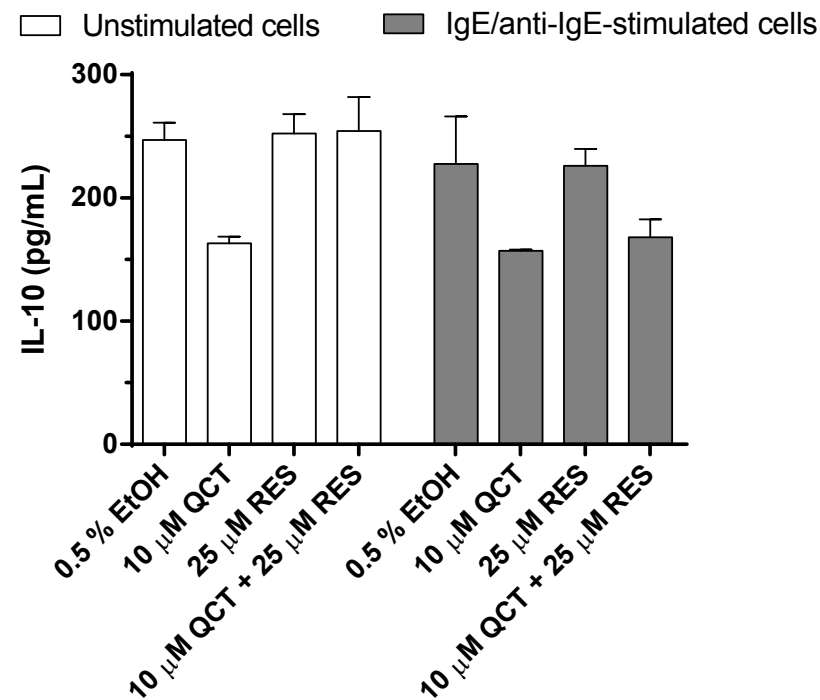

Figure 57. Effect of quercetin (QCT) (A), resveratrol (RES) (B) and QCT+RES (C) on IL-10 secretion. EtOH: ethanol. $\mathrm{N}=2$ for $A$ and $\mathrm{B}$ (single samples); and $\mathrm{N}=1$ for $\mathrm{C}$ (triplicate). 


\section{VI.4.3.7. IL-13 secretion}

Figure 58 (A-C) shows the effect of QCT, RES and their combination on IL-13 secretion. IgE/anti-IgE stimulation induced IL-13 secretion by CBMCs (Figures $58 \mathrm{~A}$ and B). QCT significantly decreased IL-13 secretion at concentrations of 10, 15 and 20 $\mu \mathrm{M}$ (all $p<0.05$; Figure $58 \mathrm{~A}$ ). IL-13 secretion was also significantly decreased when CBMCs were treated with 5, 10, 25 and $100 \mu \mathrm{M}$ RES (all $p<0.05$; Figure 58B). The combination of QCT and RES did not have any effect on IL-13 secretion (Figure 58C). 
A

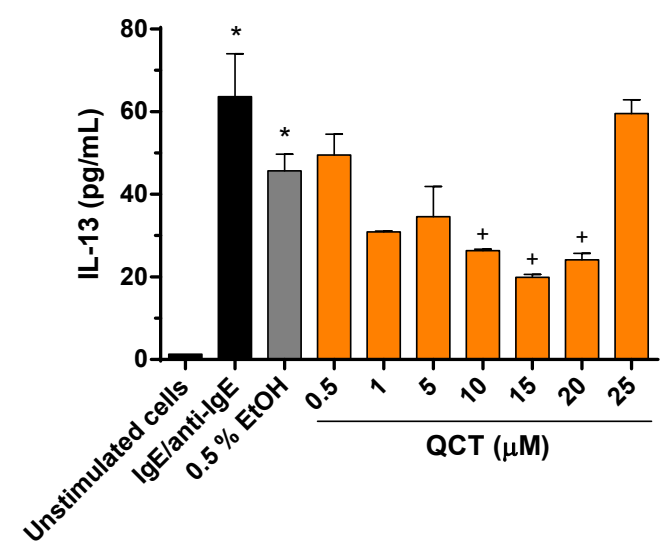

B

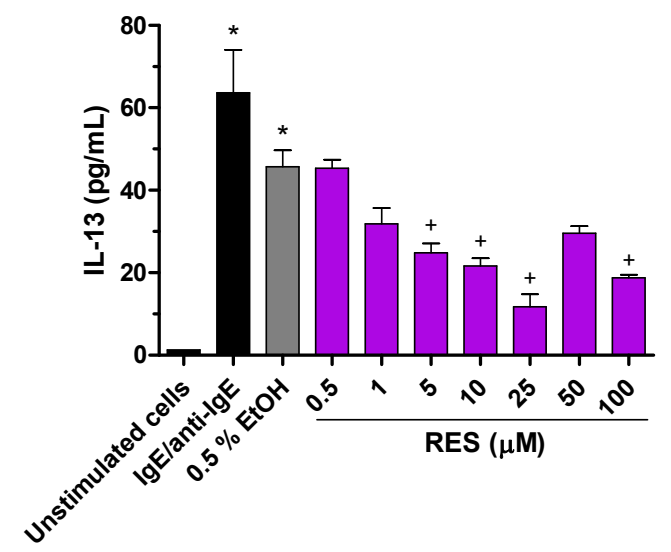

C

$\square$ Unstimulated cells $\square$ IgE/anti-lgE-stimulated cells



Figure 58. Effect of quercetin (QCT) (A), resveratrol (RES) (B) and QCT+RES (C) on IL-13 secretion. " $p<0.05$, compared to unstimulated cells; ${ }^{+} p<0.05$, compared to stimulated cells treated with ethanol (EtOH). $\mathrm{N}=2$ for $A$ and $B$ (single samples); and $\mathrm{N}=1$ for $\mathrm{C}$ (triplicate). 


\section{VI.4.3.8. TNF- $\alpha$ secretion}

Figure 59 (A-C) shows the effect of QCT, RES and their combination on TNF$\alpha$ secretion. IgE/anti-IgE stimulation induced TNF- $\alpha$ secretion by CBMCs (Figures $59 \mathrm{~A}$ and B). This stimulated secretion of TNF- $\alpha$ was significantly decreased when QCT was added at 1, 10, 15, 20 and $25 \mu \mathrm{M}$ QCT (all $p<0.05$; Figure 59A). Similar inhibitory effects on TNF- $\alpha$ secretion were found when IgE/anti-lgE-stimulated CBMCs were treated with 1, 5, 10, 25, 50 and $100 \mu \mathrm{M}$ RES ( $p<0.05$; Figure 59B). The mix of both compounds did not have any effect on TNF-a production (Figure 59C). 
A

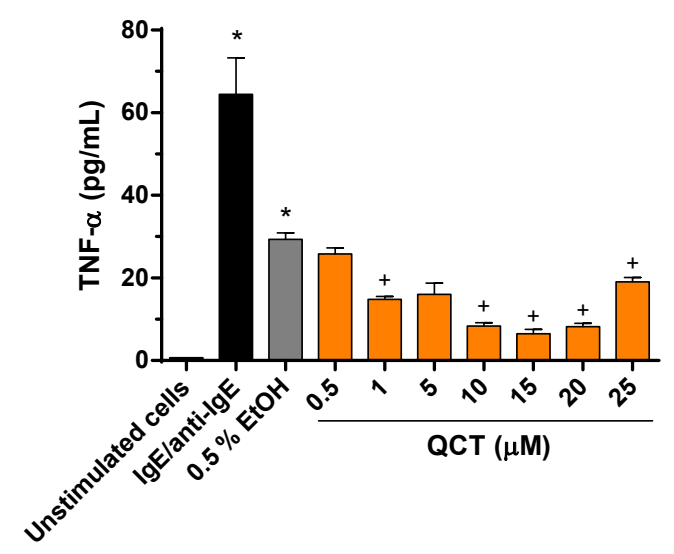

B

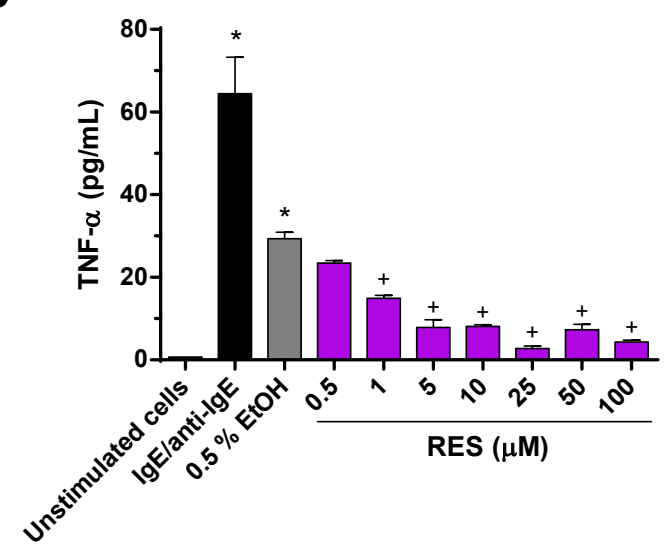

C

$\square$ Unstimulated cells $\square$ IgE/anti-lgE-stimulated cells

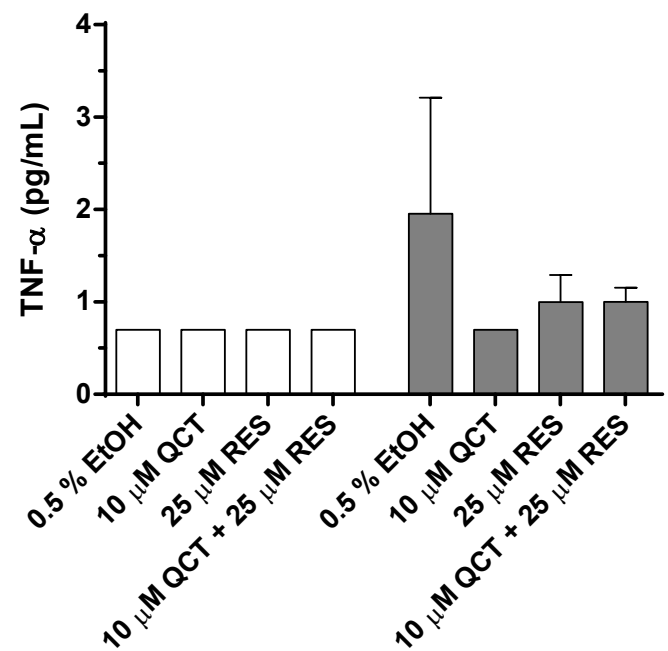

Figure 59. Effect of quercetin (QCT) (A), resveratrol (RES) (B) and QCT+RES (C) on TNF-a secretion. ${ }^{*} p<0.05$, compared to unstimulated cells; ${ }^{+} p<0.05$, compared to stimulated cells treated with ethanol (EtOH). $\mathrm{N}=2$ for $\mathrm{A}$ and $\mathrm{B}$ (single samples); and $\mathrm{N}=1$ for $C$ (triplicate). 
VI.4.3.9. IFN-y secretion

Figure 60 (A-C) shows the effect of QCT, RES and their combination on IFN-Y secretion. IFN-y secretion was not stimulated by IgE/anti-IgE in CBMCs. Neither QCT, nor RES, nor their combination had any effect on IFN-y secretion. 
A

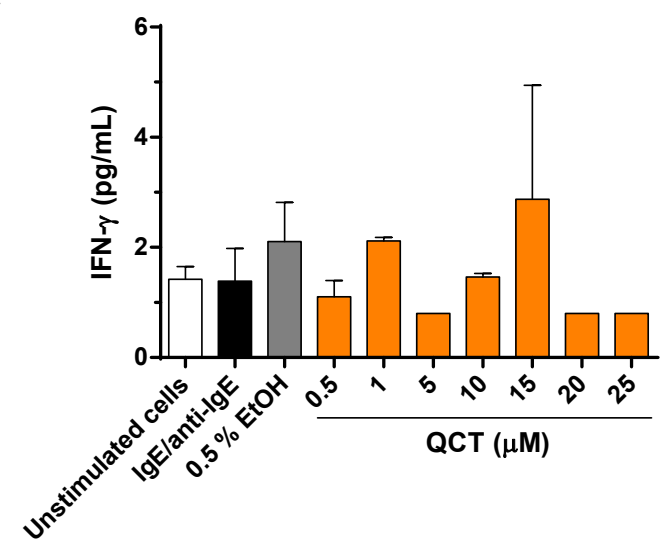

B

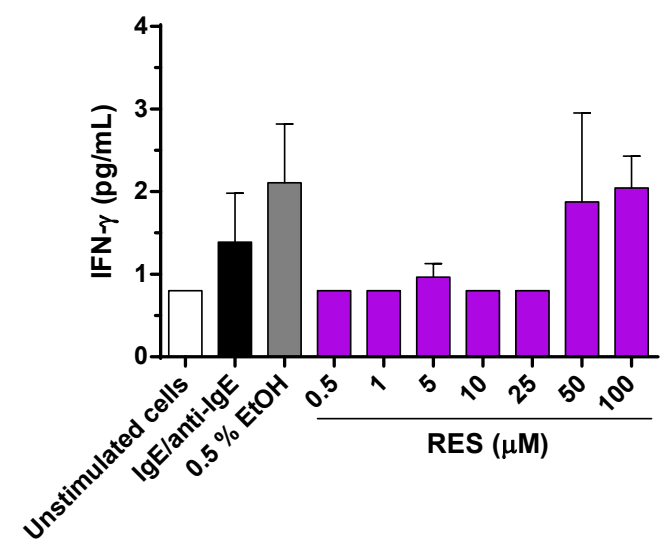

C $\square$ Unstimulated cells $\square$ IgE/anti-lgE-stimulated cells



Figure 60. Effect of quercetin (QCT) (A), resveratrol (RES) (B) and QCT+RES (C) on IFN-y secretion. EtOH: ethanol. $\mathrm{N}=2$ for $\mathrm{A}$ and $\mathrm{B}$ (single samples); and $\mathrm{N}=1$ for $\mathrm{C}$ (triplicate). 



\section{Discussion}



There are several ocular surface diseases, such as DED and allergy, in which inflammation is the underlying mechanism. It has been demonstrated that several types of cells play a key role in these inflammatory disorders. For example, it was demonstrated that pro-inflammatory cytokines in the conjunctival epithelium ${ }^{71,79,81}$ and $\mathrm{CD}^{+} \mathrm{T}$ cell infiltration into the conjunctiva and lacrimal glands are found in patients with DED. ${ }^{4,10,79}$ Regarding ocular allergic diseases, acute allergy occurs as a result of IgE-mediated mast cell degranulation, whilst chronic allergies are mediated by $\mathrm{T}$ cells and continuous activation of mast cells. ${ }^{119,120}$ This fact will help in the development of new treatments that can have a beneficial effect on the greatest number of cells involved in the inflammatory process.

The medicinal use of naturally-occurring compounds has had a great contribution to human health care, being the basis for some of today's common medicines, for example anticancer drugs. There are several sources of natural products, but interest focuses predominantly on plant sources, more specifically, on polyphenols. Two of the major polyphenols are QCT and RES because of their biological effects. These two compounds have been shown to have anticancer, antioxidant and antiviral activities, among others, but this work has focused mainly on their anti-inflammatory effects.

The aim of this thesis was to elucidate the role of both compounds and their combination as potential therapeutic agents for ocular inflammation, and more specifically, for DED and ocular allergy.

In order to confirm the hypotheses presented in this thesis, the antiinflammatory and antioxidant effects of polyphenols were firstly determined in two human ocular surface epithelial cell lines (from cornea and conjunctiva) by measuring cytotoxicity, cytokine/chemokine secretion, COX-2 expression and intracellular ROS production. After that, the immunoregulatory properties of QCT, RES and their combination were studied in-vitro on activated PBMCs from healthy donors. Their effects of cytotoxicity and on proliferation of PBMCs were determined.

Once the anti-inflammatory effect of QCT, RES and their combination was studied in-vitro, their potential therapeutic effect on DED was corroborated using a murine model of DED, followed by an adoptive transfer model. Previous studies by Dr. Stern and colleagues pointed out that this murine model of DED allows imitation of the pathophysiological processes involved in the development of DED, ${ }^{87,92-96}$ thus being a suitable in-vivo model for testing the polyphenols studied in this thesis. In addition, the major role of $\mathrm{CD}^{+} \mathrm{T}$ lymphocytes in $\mathrm{DS}$ is corroborated by the adoptive transfer model, which could help to elucidate the mechanism underlying the anti-inflammatory properties of polyphenols. 
Finally, the anti-allergy effects of both QCT and RES, and their combination was studied on sensitised/activated CBMCs. For this purpose, histamine secretion and cytokine/chemokine production by CBMCs were studied.

The use of compounds which have potent anti-inflammatory and antioxidant effects on inflammatory diseases are well documented. However, the potential effect of these compounds on ocular surface diseases has not been fully evaluated to date. This thesis has demonstrated that polyphenols, such as QCT and RES, have antioxidant and anti-inflammatory activities on cells involved in ocular surface diseases. Moreover, it has been demonstrated for the first time the immunoregulatory effect of both polyphenols in an in-vivo model of DED, inhibiting $T$ cell activation in recipients of DSexposed mice. 


\section{VII.1. In-vitro model of inflammation and oxidative stress in human conjunctival and corneal epithelial cells}

The first objective of this thesis was to determine the anti-inflammatory effect of QCT, RES and their combination on two ocular surface epithelial cell lines, IOBA-NHC and HCE cells. For this purpose, the cytotoxicity of different concentrations of QCT, RES and their combinations were firstly determined. After that, non-toxic concentrations were evaluated for their anti-inflammatory activity on cytokine/chemokine release and COX-2 expression in an in-vitro model of ocular inflammation. Additionally, their antioxidant effects were tested on an in-vitro model of UV-induced oxidative stress.

It has been shown that cell culture medium plays a key role when polyphenols are tested in-vitro, ${ }^{294}$ acting as pro-oxidant, degrading and decreasing polyphenol content. This degradation has been linked to the presence of $\mathrm{NaHCO}_{3}$ in culture medium. ${ }^{295,296}$ In addition, cell culture media usually contain many potential antioxidant compounds which can interfere with the antioxidant capacity of tested compounds, when studying oxidative stress in-vitro. Two of these compounds are pyruvate and phenol red. ${ }^{297-300}$ Therefore, in order to avoid these artefacts in the results, all experiments from this part of the thesis were carried out using a culture medium devoid of $\mathrm{NaHCO}_{3}$, sodium pyruvate and phenol red.

Some previous in-vitro studies have shown that polyphenols exhibit different cytotoxicity depending on the cell type used and/or specific type of polyphenol. The results from this thesis showed that QCT was not toxic for both IOBA-NHC and HCE cells, up to concentration of $25 \mu \mathrm{M}$. Concentrations of QCT above this value were not tested due to the very low solubility of QCT in $\mathrm{EtOH}(2 \mathrm{mg} / \mathrm{mL})$. Thus, the amount of QCT tested in this work was restricted by the vehicle. However, as showed above in the Results section of this thesis, this solubility restriction was not a limitation to testing the biological properties of QCT. Regarding RES toxicity, this compound was not toxic up to $300 \mu \mathrm{M}$ in both cell lines. Moreover, IOBA-NHC cells tolerated RES better than HCE cells, because the latter decreased more than conjunctival epithelial cells at 300 $\mu \mathrm{M}$ RES after $24 \mathrm{~h}$ exposure.

Both polyphenols were also mixed at different concentrations to test their toxicities when applied in combination. When 15, 20 and $25 \mu \mathrm{M}$ QCT were mixed with 25,50 and $100 \mu \mathrm{M}$ RES, IOBA-NHC cell numbers decreased in some combinations of 
QCT+RES, but viability remained above $80 \%$ for all treatments. On the other hand, none of the combinations tested of QCT and RES were toxic for corneal epithelial cells.

Results from the cytotoxicity assays agreed with previous work. Stoddard et al. studied the antioxidant effect of some polyphenols in stratified human corneal limbal epithelial cells and found that QCT is not toxic at $56.5 \mu \mathrm{M}$ in these cells. ${ }^{301}$ Other authors have tested different polyphenols in ocular surface epithelial cells. For example, Chen et al. found that curcumin, a natural substance derived from the rhizome of the plant Curcuma longa, is not toxic up to $30 \mu \mathrm{M}$ for immortalised human corneal epithelial cells. ${ }^{302}$ While Cavet et al. found that the green tea polyphenol epigallocatechin gallate is not toxic at any of the doses tested $(0.3-30 \mu \mathrm{M})$ for a human corneal epithelial cell line. ${ }^{303}$ Taken together, these studies suggest that polyphenols in general, and QCT and RES in particular, are not toxic for ocular surface epithelial cell lines at concentrations up to $50 \mu \mathrm{M}$.

TNF- $\alpha$ is a cytokine that has pleiotropic effects including activation of apoptosis, inflammation and immune responses, in many cell types. Thus, TNF- $\alpha$ is a potent stimulator of cytokine/chemokine release such as IL-6, IL-8/CXCL8 and IP-10/CXCL10 by ocular surface epithelial cells. ${ }^{154}$ However, not only does TNF- $\alpha$ stimulate the ocular surface epithelium, but it is also secreted by both corneal and conjunctival epithelial cells when they are stimulated by osmotic stress. ${ }^{304,305}$

In order to study the anti-inflammatory effect of polyphenols, TNF- $\alpha$ was selected as a pro-inflammatory stimulus to challenge both ocular surface epithelial cell lines. TNF- $\alpha$ was chosen as the stimulus for IOBA-NHC and HCE cells because this pro-inflammatory cytokine is involved in many ocular diseases, such as DED and ocular allergy. For example, TNF- $\alpha$ is elevated in patients with DED, ${ }^{80,113}$ Sjögren syndrome ${ }^{71}$ ocular cicatricial pemphigoid, ${ }^{306}$ and Steven-Johnson syndrome. ${ }^{307}$ In addition, TNF- $\alpha$ is able to stimulate the recruitment of leukocytes to inflamed ocular surface and activate them, by promoting expression of adhesion molecules such as ICAM-1 on the ocular surface epithelium. ${ }^{84}$ TNF- $\alpha$ is also released during acute allergic reactions by conjunctival mast cells, leading to upregulation of TNF receptor 1 (TNFR1) on human conjunctival epithelial cells, ${ }^{308}$ however it has not been detected in patients with SAC. ${ }^{309}$ Together with IL-4, TNF- $\alpha$ is a promoter of adhesion molecules expression and chemokine secretion from corneal keratocytes, conjunctival fibroblasts and epithelial cells, all contributing to inflammatory cell recruitment and induction of the allergic late phase reaction. ${ }^{310}$ In addition, it is one of a number of cytokines found in patients with $\mathrm{VKC}^{309,311}$ and $\mathrm{AKC},{ }^{312}$ especially in their most severe forms. ${ }^{139}$ 
As expected, TNF- $\alpha$ stimulated IL-6, IL-8/CXCL8 and IP-10/CXCL10 secretion by both IOBA-NHC and HCE cells after $24 \mathrm{~h}$ exposure. However, TNF- $\alpha$ did not stimulate VEGF secretion by both cell lines. IL-6, IL-8/CXCL8, IP-10/CXCL10 and VEGF secretion were analysed because all of these cytokines/chemokines are involved in some inflammatory ocular surface diseases.

IL-6 is a pro-inflammatory cytokine that has a central role in B cell differentiation and polymorphonuclear cell infiltration, ${ }^{313}$ being recognised as a primary mediator in the acute inflammatory response. ${ }^{314}$ It can be synthesised by several cell types including monocytes, $\mathrm{T}$ lymphocytes and non-inflammatory cell types, such as epithelium and endothelium. ${ }^{313,315}$ On the ocular surface, IL- 6 has an established role in inflammatory diseases, such as DED and ocular allergy. IL-6 has been suggested as a possible biomarker for DED because it is increased in the conjunctival epithelium and tears of DED patients. ${ }^{71,82,113,316,317}$ Moreover, IL-6 concentration in tears correlates with some clinical parameters in patients with DED, such as pain, tear film breakup time, Schirmer test, tear clearance, goblet cell density, tear lysozyme levels and conjunctival staining. . $^{80,82,317,318}$

IL-8/CXCL8 is a chemokine that belongs to the CXC chemokine subfamily that can be produced by any cell with a TLR (i.e. ocular surface epithelial cells and macrophages). It plays an important role in inflammation through its capacity to recruit $\mathrm{T}$ cells and non-specific inflammatory cells to sites of inflammation, and by its capacity to activate neutrophils. ${ }^{319}$ In the ocular surface, IL-8/CXCL8 is involved in ocular inflammation and angiogenesis in the conjunctiva and cornea. ${ }^{320}$ For example, patients with conjunctivochalasis have an increased level of IL-8/CXCL8 in tears, and this increase correlates with the severity of symptoms. ${ }^{321}$ In addition, IL-8/CXCL8 has also been identified in tears and conjunctiva of patients with DED. ${ }^{80,317}$ The expression of IL$8 / C X C L 8$ is increased in the conjunctival epithelium of VKC patients and is significantly correlated with the presence of neutrophils and eosinophils. ${ }^{322}$

IP-10/CXCL10 is also a CXC chemokine identified as the product of a gene induced by IFN- $\gamma \cdot{ }^{323} \mathrm{IP}-10 / \mathrm{CXCL} 10$ has been reported to attract human monocytes, $\mathrm{T}$ lymphocytes and NK cells, ${ }^{324,325}$ because CXCR3, which is the specific receptor for IP10/CXCL10, is selectively expressed on activated $T$ cells and NK cells. ${ }^{326}$ The restricted expression and the selectivity for a single receptor on $\mathrm{T}$ cells and NK cells suggest that IP-10/CXCL10 is involved in the regulation of lymphocyte recruitment and the formation of the lymphoid infiltrates observed in autoimmune inflammatory diseases on the ocular surface. For example, a high concentration of IP-10/CXCL10 is detected in tears of EDE patients, ${ }^{80}$ and also in the conjunctiva and tears of Sjögren and non- 
Sjögren syndrome DED patients. ${ }^{81}$ In addition, an increased IP-10/CXCL10 expression in both corneal and conjunctival epithelia has been described in mice exposed to DS. ${ }^{96}$

VEGF (also known as VEGF-A) is a secreted growth factor peptide that belongs to a gene family that includes VEGF-B, VEGF-C, VEGF-D, VEGF-E and placental growth factor. VEGF is critical in regulating blood and lymphatic vessel development and maintenance in health and disease. Indeed, VEGF is identified in tissues of the eye that are normally vascularised such as conjunctiva, iris and the pigment epithelium of the choroid and retina. ${ }^{327}$ Nevertheless, it plays a major role in ocular pathologies associated with tissue remodelling, inflammation and angiogenesis (growth of new blood vessels) such as pterygium, ${ }^{328}$ age-related macular degeneration and diabetic retinopathy. ${ }^{329}$

It is well known that QCT and RES have an anti-inflammatory effect on many cell types, but this effect remains unknown in ocular surface epithelial cells. In order to elucidate this question, IOBA-NHC and HCE cells were treated with different doses of QCT or RES and stimulated with TNF- $\alpha$. Regarding IOBA-NHC cells, both polyphenols QCT and RES inhibited the secretion of cytokines/chemokines tested. QCT decreased IL-6 (15, 20 and $25 \mu \mathrm{M})$, IL-8/CXCL8 (20 and $25 \mu \mathrm{M})$, and IP-10/CXCL10 (10, 15, 20 and $25 \mu \mathrm{M})$. For RES treatments, this compound also decreased IL-6 (25 and $50 \mu \mathrm{M})$, IL-8/CXCL8 $(50 \mu \mathrm{M})$ and IP-10/CXCL10 $(10,25$ and $50 \mu \mathrm{M})$. These data showed that low concentrations of both polyphenols $(10 \mu \mathrm{M})$ were sufficient to decrease IP. 10/CXCL10 secretion, whilst higher concentrations of QCT and RES were necessary to reduce IL-6 and IL-8/CXCL8 levels from conjunctival cells. In addition, although both compounds inhibited cytokine/chemokine secretion, QCT was more effective because the same inhibitory effect was reached at low doses, compared to doses of RES (i.e. QCT inhibited IL-8/CXCL8 at $25 \mu \mathrm{M}$, whilst RES had a similar effect at $50 \mu \mathrm{M}$ ).

Referring to HCE cells, QCT and RES also decreased the secretion of cytokines/chemokines tested. QCT decreased IL-6 (1, 5, 10, 15, 20 and $25 \mu \mathrm{M})$, IL8/CXCL8 (5, 10, 15, 20 and $25 \mu \mathrm{M})$, and IP-10/CXCL10 (1, 5, 10, 15, 20 and $25 \mu \mathrm{M})$; and RES decreased IL-6 (10, 25 and $50 \mu \mathrm{M})$, IL-8/CXCL8 $(50 \mu \mathrm{M})$, and IP-10/CXCL10 $(0.5,1,5,10,25$ and $50 \mu \mathrm{M})$. Both compounds significantly inhibited IL-6 and IP10/CXCL10 dose-dependently from low doses. IL-8/CXCL8-stimulated secretion was only inhibited by QCT dose-dependently, whilst only a high dose of RES (50 $\mu \mathrm{M})$ was able to inhibit IL-8/CXCL8. In contrast to conjunctival epithelial cells, QCT and RES had similar inhibitory effect on HCE cells because low doses of both compounds decreased cytokine/chemokine secretion (IL-6 and IP-10/CXCL10). This indicates that the antiinflammatory effects of QCT and RES on IL-6 and IP-10/CXCL10 secretion is stronger 
in HCE cells than IOBA-NHC cells, and QCT is more effective that RES in inhibiting IL8/CXCL8 secretion by both cell lines.

Interestingly, $25 \mu \mathrm{M}$ QCT decreased cytokine/chemokine concentrations (IL-6, IL-8/CXCL8 and IP-10/CXCL10) to control values in both cell lines, although significant differences were found in some data when compared to controls. This is due to the fact that there was a different amount of variability or spread, in the dataset. Data from cells exposed to TNF- $\alpha$ (treated or not with polyphenols) had higher variability than those found in control samples (cells not exposed to TNF- $\alpha$ ). This may be solved by increasing the number of experiments performed.

Once the effects of QCT and RES were tested separately, both polyphenols were mixed and the anti-inflammatory properties of their combination were studied in both cell lines. For this purpose, one low dose from each compound was chosen based on dose-response curves: the concentrations selected were $0.5 \mu \mathrm{M}$ QCT and $5 \mu \mathrm{M}$ RES, because neither concentration had a significant effect on almost all cytokines/chemokines studied (except for RES on IP-10/CXCL10 secretion by HCE cells). Results from this thesis showed that the combination $0.5 \mu \mathrm{M} Q C T+5 \mu \mathrm{M}$ RES decreased stimulated secretion of IL-6 and IL-8/CXCL8 by IOBA-NHC cells. Although the inhibitory effect of $0.5 \mu \mathrm{M}$ QCT $+5 \mu \mathrm{M}$ RES on IL-8/CXCL8 was similar to that provoked by $0.5 \mu \mathrm{M}$ QCT alone, in this case no significant difference was found when compared to the control group. In addition, $0.5 \mu \mathrm{M}$ QCT did not show any effect in the dose-response curve, whilst it did in the experiment using the combination of polyphenols. The combination of QCT and RES inhibited IP-10/CXCL10 stimulated secretion by cells to a greater extent than both polyphenols did separately. Although this inhibition did not reach statistical significance compared to TNF- $\alpha$-stimulated cells treated with vehicle.

Referring to HCE cells treated with the combination of polyphenols, the inhibition of cytokine/chemokine secretion was not significantly different from that provoked by polyphenols alone. These results suggest that QCT and RES are effective in inhibiting multiple cytokines and chemokines induced by a pro-inflammatory mediator (TNF- $\alpha$ ), demonstrating that they might have a therapeutic potential in the treatment of ocular surface inflammatory diseases. A stronger inhibitory effect was observed in the conjunctival epithelial cells when treated with the combination of low doses of polyphenols, but this effect was not found in corneal epithelial cells. Thus, it is hypothesised that other combinations may have different effect compared to those found for $0.5 \mu \mathrm{M} Q \mathrm{QCT}+5 \mu \mathrm{M}$ RES.

Although there are very few studies regarding the effects of natural compounds on the ocular surface, similar results to those described in this thesis have been 
described for other polyphenols in ocular surface epithelial cells. Chen et al. demonstrated that curcumin has an anti-inflammatory effect on HCE cells exposed to increasing osmolarities of cultured medium (with sodium chloride, $\mathrm{NaCl}$ ). ${ }^{302}$ They found that $5 \mu \mathrm{M}$ curcumin can abolish the hyperosmoticity-induced production of cytokines such as IL-1 $1 \beta$, IL- 6 and TNF- $\alpha$, as well as IL-1 $\beta$ mRNA and activation of p38. Therefore, they suggested that curcumin inhibits hyperosmoticity-induced increase of IL-1 $\beta$ production in HCE cells, through inhibition of $p 38$, which leads to NF-kB p65 inhibition by curcumin. Cavet et al. also described that epigallocatechin gallate has an anti-inflammatory effect on HCE cells using two stimuli, IL-1 $\beta$ and hyperosmolarity with sucrose. ${ }^{303}$ The authors found that IL-1 $\beta$ increases the release of IL-6, IL-8/CXCL8, MCP-1, G-CSF and GM-CSF, whereas hyperosmolarity only increases IL-6 and MCP1. The polyphenol epigallocatechin gallate decreases IL-1 $\beta$ - and hyperosmolar-induced cytokine/chemokine secretion by HCE cells, in a dose-dependent manner from 3 to 30 $\mu \mathrm{M}$. In addition, this polyphenol inhibits the IL-1 $\beta$ - and hyperosmolar-induced phosphorylation/activation of both p38 and JNK in HCE cells. Their results also indicated that inhibition of IL-1 $\beta$-induced cytokine expression is, at least in part, mediated by inhibition of both AP-1 and NF-kB transcription activities by epigallocatechin gallate.

The present study has shown that TNF- $\alpha$ did not provoke VEGF secretion by any of both epithelial cell lines, although it has been demonstrated that normal and inflamed ocular surface epithelial cells can secrete VEGF. ${ }^{330,331}$ For example, VEGF levels are increased in tears of mild-to-moderate DED patients ${ }^{80}$ and in conjunctival biopsies and tears of patients suffering $\mathrm{VKC}$ or $\mathrm{AKC},{ }^{332}$ playing a crucial role in the remodelling process of these severe allergic conjunctival disorders. ${ }^{333}$ Yet, the effect of QCT and RES on VEGF secretion by ocular surface epithelial cells could not be determined in this in-vitro model. However, previous studies have reported that QCT and RES can affect VEGF production in other cell types. For example, stimulation of human retinal pigment epithelial cells (ARPE-19) with glucose in the presence of 0-10 $\mu \mathrm{M}$ RES dose-dependently inhibits VEGF, along with IL-6, IL-8/CXCL8 and COX-2. ${ }^{334}$ Similar results were found when human retinal pigment epithelial cells from donors were stimulated with platelet-derived growth factor or cobalt chloride in the presence of 0-50 $\mu \mathrm{M}$ QCT, as QCT also inhibit VEGF in a dose-dependent manner. ${ }^{335}$ In addition to that, it has been demonstrated that polyphenols can also inhibit VEGF in-vivo. Koh et al. evaluated the efficacy of topical application of the green tea polyphenol epigallocatechin gallate for the treatment of corneal neovascularisation in a rabbit model. They induced neovascularisation by placing a black silk suture in the corneal 
stroma for a week. After that, they treated rabbits with topical epigallocatechin gallate for 2 weeks. Their results showed that epigallocatechin gallate effectively inhibits corneal neovascularisation in rabbits by decreasing VEGF messenger RNA levels, along with a decrease of COX-2 expression. ${ }^{336}$ Our in-vitro model of inflammation demonstrated that TNF- $\alpha$ did not induce VEGF secretion by conjunctival and corneal epithelial cells. However, VEGF levels are increased in some ocular diseases as it has been pointed out above and it is hypothesised that both conjunctival and corneal epithelial cells may secrete VEGF using other stimuli such as hypoxia, ${ }^{337}$ which could also permit the study of the effect of polyphenols on VEGF secretion by ocular surface epithelial cells.

Besides cytokines and chemokines, prostaglandins and thromboxanes (collectively termed prostanoids) play an important role in the generation of the inflammatory response. ${ }^{338}$ They are formed when arachidonic acid is released from the plasma membrane by phospholipases and is metabolised by the sequential actions of enzymes. These are bifunctional enzymes (having both cyclooxygenase and peroxidase activities) and exist as two isoforms referred to as COX-1 and COX-2. ${ }^{339}$ COX-1 is expressed constitutively in most cells, being the dominant source of prostanoids that subserve housekeeping functions, such as gastric epithelial cytoprotection and homeostasis. ${ }^{340}$ COX-2 is the more important source of prostanoid formation in inflammation and in proliferative diseases, being induced by inflammatory stimuli, hormones and growth factors. ${ }^{340}$ COX-2 is also involved in inflammatory disorders of the eye. For example, samples from patients suffering pterygium have COX-2 expression, while normal conjunctiva and limbus specimens do not express COX-2, indicating that COX-2 only exists in the pterygium and not in the normal conjunctiva and limbus. ${ }^{341}$ Increased levels of prostaglandins in tears are found in patients with DED, which correlates with patients' symptom scores. ${ }^{342}$ These clinical results are supported by increased COX-2 and prostaglandins expression levels in tear-producing tissues of DED mice. ${ }^{342}$ In addition, it seems that prostaglandins, and therefore COX-2, are involved in allergic conjunctivitis as non-steroidal antiinflammatory therapy, which inhibits COX enzymes, is commonly used to alleviate itching associated with allergic conjunctivitis. ${ }^{343}$

In order to complement the study of the anti-inflammatory properties of polyphenols, the effects of QCT and RES on basal and TNF- $\alpha$-stimulated COX-2 expression in both epithelial cell lines, were analysed. These results showed that TNF$\alpha$ induced COX-2 expression in IOBA-NHC cells. Regarding the effect of polyphenols, neither QCT, nor RES, nor their combination had any effect on COX-2 expression in 
IOBA-NHC cells, compared to stimulated cells treated with $0.5 \% \mathrm{EtOH}$. However, COX-2 levels expressed as a fold-change with respect to each control showed that QCT and QCT+RES decreased COX-2 expression but not significantly. Surprisingly, $25 \mu \mathrm{M}$ QCT induced an increase of COX-2 expression in both unstimulated and stimulated IOBA-NHC cells, but this increase was less than control cells when the fold change was calculated. This unexpected result may suggest a pro-inflammatory role of QCT, however results from this thesis and scientific literature have shown that QCT is a COX inhibitor compound in several types of cells. For example, Qureshi et al. found that QCT inhibits LPS-induced expression of COX-2 in peritoneal macrophages from mice. ${ }^{259}$ Similar results were reported by García-Mediavilla et al. who found that QCT produces a large concentration-dependent decrease of COX-2, as well as inhibition of mRNA levels, in Chang liver cells. ${ }^{262}$

Referring to HCE cells, TNF- $\alpha$ did not induce COX-2 expression in corneal cells. This is a notable limitation of this in-vitro assay because TNF- $\alpha$ did not stimulate a positive control in order to study the effect of polyphenols on COX-2 expression by HCE cells. Thus, comparison of how polyphenols change COX-2 expression relative to vehicle may not be relevant.

Nevertheless, results from this thesis demonstrated that QCT, RES and a mix of both compounds decreasing TNF- $\alpha$-induced COX-2 production by conjunctival epithelial cells.

In this work it has also been demonstrated that polyphenols can protect conjunctival and corneal epithelial cells from UV-B radiation-induced oxidative stress. IOBA-NHC and HCE cells were irradiated with UV-B light for $15 \mathrm{~s}\left(107.25 \mathrm{~mJ} / \mathrm{cm}^{2}\right)$. The average value of solar UV-B radiation reaching the human cornea is $105 \mathrm{~mJ} / \mathrm{cm}^{2}$ during a $1 \mathrm{~h}$ exposure. ${ }^{344}$ Thus, the irradiance of the lamp used in this experiment was energetically relevant as the energy of $1 \mathrm{~h}$ exposure was condensed to $15 \mathrm{~s}$. As expected, this UV-B radiation provoked an increase of intracellular ROS production in ocular surface epithelial cells. ${ }^{280}$

UV radiation is one of the largest causes of ROS induction in the eye and plays an important role in the pathogenesis of a large number of ocular diseases. For example, one of the most common acute effects of UV-B radiation on the cornea is photokeratitis. ${ }^{345,346}$ Chronic UV radiacion can also provoke solar keratopathy, ${ }^{347}$ pterygium, ${ }^{348,349}$ and ocular melanoma in the conjunctiva. ${ }^{347,350}$ Oxidative stress, along with inflammation, are involved in all of these pathologies. Cytokines, such as IL-6 and IL-9, are released by the epithelial cells in pterygium after UV-B exposure ${ }^{349}$ and oxidative mediators have also been found in patients with primary pterygium. ${ }^{351}$ In-vivo 
models of photokeratitis have demonstrated that there is a decrease in antioxidant enzymes in the corneal epithelium, when eyes are excessively exposed to UV-B. This event provokes an oxidative stress, leading to transcriptional activation of inflammatory factors such as NF-KB and iNOS, and formation of cytotoxic nitric oxide and nitrogenrelated oxidants. ${ }^{352-354}$

When IOBA-NHC and HCE cells were exposed to UV-B and treated with different doses of QCT or RES, ROS production decreased in a dose dependentmanner in both cell lines. This effect was stronger in HCE cells than that found in IOBA-NHC, suggesting that polyphenols are more effective at protecting corneal epithelial cells from UV-B light. Moreover, the antioxidant effect of QCT was stronger than the antioxidant effect of RES in HCE cells: a lower concentration of QCT (1 $\mu \mathrm{M})$ was necessary for decreasing ROS production significantly, compared to RES ( $25 \mu \mathrm{M})$. In addition, the mix of both compounds abolished the significant increase of intracellular ROS production in both cell lines, compared to UV-B unexposed cells. These results indicate that QCT, RES and the mix of low doses of both compounds can decrease the oxidative stress involved in cells exposed to UV-B radiation. These results agree with previous studies. For example, the antioxidant effect of QCT was previously described by Stoddard et al. in stratified human corneal limbal epithelial cells. ${ }^{301}$ Although they did not expose cells to UV-B radiation, they found that the concentration of QCT which gives $50 \%$ of the maximum response (EC50) is $2.98 \mu \mathrm{M}$ QCT, when cells are exposed to 2,2'-azobis(2-amidinopropane) dihydrochloride (to generate intracellular ROS).

Regarding the antioxidant effect of other polyphenols on ocular surface epithelial cells exposed to UV radiation, similar results were described by Larrosa et al. using two phenolic compounds: hydrocaffeic acid and p-coumaric acid. ${ }^{355}$ They found that $10 \mu \mathrm{M}$ hydrocaffeic acid alone and a mixture of both compounds (5 $\mu \mathrm{M}$ hydrocaffeic acid $+5 \mu \mathrm{M}$-coumaric acid) reduce oxidation damage in human conjunctival cells exposed to UV-B radiation $\left(4.4 \mathrm{~mJ} / \mathrm{cm}^{2}\right)$. In addition, they also treated rabbit eyes with hydrocaffeic acid alone and a mixture of both compounds drops before UV-B exposure $\left(7.9 \mathrm{~mJ} / \mathrm{cm}^{2}\right)$, and showed that polyphenols reduce corneal and scleral DNA oxidation damage, xanthine oxidase activity and MDA levels in the corneal tissues of rabbits. Similar results have been reported recently by Chen et al. ${ }^{356}$ They exposed rabbits to UV-B radiation $\left(100 \mathrm{~mJ} / \mathrm{cm}^{2}\right)$ and further treated them with topical epigallocatechin gallate $(0.01 \%$ and $0.1 \%)$ for 8 weeks. Treatment with epigallocatechin gallate eye drops ameliorates corneal damage, as well as increasing SOD, catalase and GSH activity in the corneas, compared to the UV-B-treated group. 
Corneal and conjunctival epithelial cells play a major role in ocular surface inflammation. Both types of cells can secrete inflammatory mediators when they are challenged by pro-inflammatory stimuli. This thesis has demonstrated for the first time that two naturally-occurring compounds, QCT and RES, and their combination modulate the inflammatory response of ocular surface epithelial cells in-vitro, decreasing cytokine/chemokine release when epithelial cells were exposed to TNF- $\alpha$. Both polyphenols, QCT and RES, have shown to have similar effect on both ocular surface epithelial cells, but QCT is more effective than RES. In addition, the results described in this thesis have also demonstrated that QCT and RES can protect epithelial cells from UV-B-induced oxidative stress. The chronic inflammation is linked to the oxidative stress because this has been evidenced in ocular surface diseases, such as DED and ocular allergy. Results from this thesis suggest that QCT and RES may exert their protective effect via both pathways. This is an important and relevant result because a novel therapy using polyphenols may act as both an anti-inflammatory antioxidant therapy when applied topically to the ocular surface. 


\section{VII.2. In-vitro proliferative response of human T cells}

One of the therapeutic objectives in tissue inflammation is to reduce the local inflammatory response by regulation of $\mathrm{T}$ cell activation and proliferation and cytokine production. $\mathrm{CD}^{+} \mathrm{T}$ cells play a key role in several ocular surface diseases such as DED and allergy. Thus, after testing the anti-inflammatory effect of polyphenols in ocular surface epithelial cells, the second objective of this thesis was to test the immunoregulatory effect of QCT, RES and their combination on PBMCs from healthy donors. For this purpose, the cytotoxicity of polyphenols was firstly determined and thereafter the antiproliferative activity of QCT, RES and QCT+RES was tested on PBMCs.

Results from this thesis have demonstrated that cytotoxicity of QCT, RES and QCT+RES increased in a dose-dependent manner. It was also demonstrated that the polyphenol combination decreased proliferation of activated PBMCs, without affecting their viability. In this in-vitro model of inflammation, PBMCs from healthy donors were activated using anti-CD3/anti-CD28 antibodies.

A wide range of agents can be used to induce $T$ cell activation and proliferation, such as calcium ionophore combined with phorbol ester and lectins (phytohemagglutinin (PHA) and concanavalin A). However, a direct way of inducing $\mathrm{T}$ cell activation involves stimulation with monoclonal antibodies that interact with the TCR/CD3 complex. This method, which was used in this thesis, has the advantage that it is a more physiologically relevant approach to stimulate $\mathrm{T}$ cells in a manner that partially imitates stimulation by APCs. Thus, the in-vitro model used in this thesis for activating PBMCs attempts to simulate immune-complex-mediated activation and proliferation of $\mathrm{T}$ cells following the two-signal model of lymphocyte activation, which has prevailed as a dominant paradigm to explain how $T$ and $B$ cells could be constrained into discriminating self from non-self. ${ }^{357}$

In order to test the anti-inflammatory effect of polyphenols, activated PBMCs were treated for $96 \mathrm{~h}$ with a wide range of QCT, RES or QCT+RES concentrations to determine which concentrations were toxic for PBMCs. The results showed that high doses of RES decreased activated PBMC numbers, whilst none of the QCT concentrations tested decreased cell viability. In contrast, QCT and RES combinations increased cytotocity when the concentration of both polyphenols increased. This indicates that RES is more toxic than QCT for PBMCs. 
Once the cytotoxicity of polyphenols was tested, low concentrations of QCT and RES were studied individually and in combination, in order to discern whether polyphenols could affect cell proliferation after $120 \mathrm{~h}$, using the CFSE assay counterstained with PI. Results showed that $5 \mu \mathrm{M}$ RES in combination with 5 and 10 $\mu \mathrm{M}$ QCT, and $10 \mu \mathrm{M}$ RES in combination with $0.5,1,5$ and $10 \mu \mathrm{M}$ QCT decreased PBMC proliferation, whilst only $10 \mu \mathrm{M}$ RES showed a tendency to decreased cell proliferation. This is the most relevant result from this part of the thesis because it demonstrates the antiproliferative effect of QCT and RES on PBMCs. Moreover, this effect on proliferation was not due to a decrease in cell viability as PI staining demonstrated. More studies are necessary to elucidate whether other concentrations of QCT and RES, and other combinations of both compounds are more effective.

It is necessary to emphasise that the cytotoxicity experiments and the viability data from the proliferation assays were not comparable to each other, although they both showed PI negative cells. This is due to the fact that samples from the cytotoxicity assay were gated at the end of the experiment (96 h) and compensation was performed pre-acquisition by the instrument, prior to analysing all samples. In contrast, the CFSE assay was gated at day 0 and compensation was performed post-acquisition using specific software (WinList software) after $120 \mathrm{~h}$ of culture. All these circumstances make it very difficult to compare to both experiments. For example, some concerns may arise comparing the results from the cytotoxicity assay against the viability results from the proliferation assay: the combination of 5 and $10 \mu \mathrm{M}$ RES with 5 and $10 \mu \mathrm{M}$ QCT increased cytotoxicity significantly (Figure 33), whereas the same polyphenol combinations did not decrease cell viability in the proliferation assay as shown in Figure 34B. In addition, low doses of RES $(0.5$ and $1 \mu \mathrm{M})$ in combination with QCT were tested in the first experiment $(n=1)$, along with 5 and $10 \mu M R E S$, but only these latter concentrations diminished proliferation (without affecting cell viability). Therefore, only 5 and $10 \mu \mathrm{M}$ RES in combination with QCT were tested subsequently. Nevertheless, it was demonstrated that the combination of low doses of QCT and RES can inhibit PBMC proliferation in-vitro.

In addition, some data from the cytotoxicity and proliferation assays were above $100 \%$. This effect was due to the fact that all data were normalised to stimulated (no treated) cells (representing $100 \%$ ). Thus, values above $100 \%$ only indicated that these values were higher that those found in stimulated (no treated) cells.

The antiproliferative effect in-vitro of QCT and RES individually on PBMCs has been reported in the scientific literature. For example, Lugli et al. ${ }^{358}$ stimulated CFSElabelled PBMCs with PHA for $72 \mathrm{~h}$, adding QCT $(10,50$ and $100 \mu \mathrm{M})$ at 24 or $48 \mathrm{~h}$ after 
stimulation. They found that $10 \mu \mathrm{M}$ QCT (added $48 \mathrm{~h}$ after PHA stimulation) slightly inhibited cell proliferation, while 50 and $100 \mu \mathrm{M}$ concentrations are much more efficient at decreasing cell proliferation. This effect is more consistent when QCT is added $24 \mathrm{~h}$ after PHA stimulation. In contrast to these results, Sternberg et al. ${ }^{359}$ stimulated PBMCs isolated from healthy donors with PHA in the presence of QCT $(0,0.2,1,5,10,25,50$, 100 and $200 \mu \mathrm{M})$ and found that QCT inhibits PBMC proliferation in a dose-dependent manner after $48 \mathrm{~h}$ of culture. The effect of QCT on cell proliferation is significant from the $5 \mu \mathrm{M}$ concentration. In addition, QCT (at a concentration of $200 \mu \mathrm{M}$ ) reduces cell proliferation by $35 \%$, with no cytotoxicity (determined using the trypan blue dye exclusion test). Moreover, the antiproliferative activity of QCT was also reported in other normal cell types such as human umbilical vein cord blood cells ${ }^{360}$ and normal CD34 ${ }^{+}$bone marrow progenitors in-vitro. ${ }^{361}$

Regarding the immunomodulation role of RES, Falchetti et al. studied its effect on PBMC proliferation and viability. ${ }^{362}$ They stimulated PBMCs with anti-CD3 for 3 days and further stimulated cells with anti-CD28 for 3 days. PBMCs were treated with RES $(0-44 \mu \mathrm{M})$ at day 1 and 4 . They found that RES (22 and $44 \mu \mathrm{M})$ completely blocks antiCD3/anti-CD28-induced PBMC proliferation with no significant effect on cell viability, whereas RES has no effect on cell proliferation at lower concentrations. Other authors reported similar results. For example, Boscolo et al. ${ }^{363}$ stimulated PBMCs with PHA in the presence of $0.1,10$ and $100 \mu \mathrm{M}$ RES and analysed cell proliferation after $72 \mathrm{~h}$, using 5-bromo-2'-deoxyuridine. This study showed that $100 \mu \mathrm{M}$ RES strongly inhibits PHA-stimulated PBMC proliferation, but it does not exert this effect at $10 \mu \mathrm{M}$. Larbi et al. also reported similar results. ${ }^{364}$ They stimulated CFSE-labelled PBMCs with antiCD3/anti-CD28 coated beads and treated them with RES $(5,10,25$ and $50 \mu \mathrm{M})$ under hyperoxic conditions (culture in air) for 6 days. They found that RES has an inhibitory effect on $\mathrm{T}$ cell activation by anti-CD3/anti-CD28-coated beads at 25 and $50 \mu \mathrm{M}$ concentrations, but not at 5 and $10 \mu \mathrm{M}$. However, this proliferation decrease is due to increased RES-induced apoptosis of PBMCs, explaining their decreased proliferation effect under hyperoxic conditions. In addition, it has been suggested that RES may exert its antiproliferative effect by down-regulating the expression of CD28 and CD80, as previously described in concanavalin A-stimulated splenocytes from mice. ${ }^{214}$ Based on these published results, it might be hypothesised that QCT and RES could increase their antiproliferative effect on anti-CD3/anti-CD28-stimulated PBMCs at concentrations higher than those used in this thesis.

Results from this thesis have shown that the combination of QCT+RES at low doses was able to decrease proliferation of anti-CD3/anti-CD28-stimulated PBMCs more than QCT and RES separately. Thus, a mix of low doses of QCT and RES can 
be more effective than the same concentration of polyphenols separately, as an immunomodulatory treatment in-vitro. Some studies also point out that mixing of polyphenols can affect cell proliferation at low concentrations. For example, Cosentino et al. studied the effect of Achyrocline satureioides (an aromatic herb from South America) infusion on PBMC proliferation. ${ }^{365}$ They tested a range of total polyphenol concentrations expressed as QCT equivalents $(0.0006-0.24 \mu \mathrm{g} / \mathrm{mL}$ QCT equivalents 0.002-0.79 $\mu \mathrm{M}$ QCT equivalents), because QCT is one of the main flavonoids contained in Achyrocline satureioides. ${ }^{366}$ This group found that treatment of PHAstimulated PBMCs from healthy donors reduces concentration-dependent PHAinduced proliferation to complete inhibition in the 0.2-0.79 $\mu \mathrm{M}$ QCT equivalent concentration range (after $48 \mathrm{~h}$ of culture), without affecting cell viability. The authors concluded that the effect exerted by Achyrocline satureioides infusion at low concentrations seems unlikely to depend solely upon the activity of QCT, because Achyrocline satureioides has shown the presence of many flavonoids, terpenoids and other chemicals. ${ }^{366}$

In this thesis, it has been demonstrated that a combination of QCT and RES exert an inhibitory activity on normal immune functions, such as $\mathrm{T}$ cell activation and proliferation. This result showed that a combination of low doses of QCT+RES have a stronger antiproliferative effect on PBMCs than both compounds alone. More studies are necessary in order to elucidate whether this effect also occurs at higher doses.

The reduction in cell proliferation by QCT+RES is an important observation, since circulating autoreactive $T$ cells in inflammatory ocular surface diseases may initiate a second cycle of inflammation, which subsequently may lead to the induction of a chronic process. 


\section{VII.3. Experimental murine model of dry eye and adoptive transfer mode}

The results from the in-vitro experiments have shown the potential of QCT, RES and QCT+RES as an anti-inflammatory therapy on different types of cells, such as conjunctival and corneal epithelial cells and PBMCs. These cells, among others, play an important role in several immune-based ocular surface diseases such as DED. Thus, the third objective of this thesis was to test QCT, RES and QCT+RES combination in an in-vivo model of DED in order to confirm their anti-inflammatory activities.

The DED model used for this thesis has been extensively studied and characterised because the mice develop ocular surface lesions similar to those found in DED patients. Mice exposed to DS have an increased CFS, reduced tear production, decreased goblet cell density and $\mathrm{CD}^{+} \mathrm{T}$ cell infiltration in conjunctiva, including an increase of cytokine/chemokine concentrations in tears. ${ }^{87,93-96,99,367}$

In this thesis, DS induced a significant decrease in tear production, as well as CFS in donor mice, as previously described. ${ }^{87}$ In contrast to the literature, however, DS did not affect either goblet cell numbers or $\mathrm{CD}^{+} \mathrm{T}$ cell infiltration of the conjunctiva. As suggested by Dr. Stern (personal communication), it may be hypothesised that it is due to the fact that the mouse exposed to DS has the complication of a fluctuating innate response that is superimposed over the adaptive immune result.

Prior to testing natural compounds in-vivo, the concentrations for both compounds were chosen based on published studies and a preliminary study carried out in Allergan in 2011. Cavet et al. ${ }^{303}$ and Lee et al. ${ }^{368}$ tested the effect of the green tea polyphenol epigallocatechin gallate in-vitro (using HCE cells) and in-vivo (in a DED model), respectively. Cavet et al. found that epigallocatechin gallate has a significant anti-inflammatory effect at $30 \mu \mathrm{M}$ on HCE cells. Results from in-vitro studies of this thesis have shown that similar concentrations of QCT and RES, to that found by Cavet et al., also had an anti-inflammatory effect on HCE cells. In addition, Lee et al. found that epigallocatechin gallate has a significant anti-inflammatory effect on DED model at a concentration of $0.01 \%$. Thus, it was hypothesised that a similar concentration of QCT and RES might also show their anti-inflammatory properties in the DED model. As a result, $0.01 \%$ QCT, $0.01 \%$ RES and their combination were tested in a preliminary study using an in-vivo model of DED (the most important result from this experiment is shown in the patent derived from this thesis in Appendix I). Based on this preliminary 
experiment, it was finally decided for experiments in this thesis to test $0.01 \%$ QCT and increase RES concentration up to $0.1 \%$, and to test RES alone and in combination with $0.01 \%$ QCT.

This part of the thesis has demonstrated that topical application of $0.01 \%$ QCT, $0.1 \%$ RES or $0.01 \%$ QCT $+0.1 \%$ RES can decrease the clinical signs (CFS) and inflammatory response (cytokine production) of the ocular surface in a murine model of DED.

CFS is a routine clinical test for determining the corneal epithelial integrity in patients. When corneal epithelial cells are damaged (leading to cell-cell junction disruption), fluorescein uptake occurs providing a visible staining. ${ }^{369}$ This disruption renders the ocular surface susceptible to environmental insults. Corneal staining is usually found in patients suffering ocular surface diseases such as DED, and even in patients exposed to a controlled adverse environment, ${ }^{66}$ which points out that the corneal epithelium is compromised. Current results from this study showed that topical treatment with $0.01 \%$ QCT and $0.01 \%$ QCT + $0.1 \%$ RES decreased clinical signs associated with corneal staining, thus protecting the ocular surface by enhancing DSexposed mouse corneal integrity. Surprisingly, these results did not correlate to TP because neither QCT nor QCT+RES had any effects on tear production. As it has been pointed out previously in this thesis based on scientific literature, DS and scopolamine induce clinical disease through different mechanisms. ${ }^{88}$ As a result, the CFS score increases for both mechanisms but DS only causes a slight tear reduction at the early time points (days 3 and 7 ) that is not conserved at later time points (days 10 and 14), whilst scopolamine inhibits tear secretion at day $10{ }^{88}$ Moreover, CFS score increases more in mice without lacrimal glands (extraorbital lacrimal gland excision) exposed to DS, than mice treated with scopolamine exposed to DS. According to Chen et al., ${ }^{88}$ these results correlate to a higher Th17 response in lacrimal gland excision+DS mice, compared to scopolamine+DS mice, indicating that Th17 is involved in regulating corneal integrity. Thus, it is hypothesised that the protective effect of QCT and RES on corneal integrity may be due to an inhibitory effect upon Th17 cells. More studies are necessary to elucidate the mechanisms underlying the improvement of corneal integrity by polyphenols.

This protective effect of studied polyphenols on corneal integrity agrees with a previous study by Lee et al. ${ }^{368}$ They tested the effect of the green tea polyphenol epigallocatechin gallate in a similar in-vivo DED model. They applied $0.01 \%$ and $0.1 \%$ epigallocatechin gallate solutions topically to both mouse eyes twice daily starting $48 \mathrm{~h}$ after DS induction. They found that topical treatments at day 4 , including vehicle $(1 \%$ 
DMSO in PBS), have a significant decrease in corneal staining compared to the untreated group, but they also reported that there is no difference in corneal staining among these treatment groups. However, after 9 days of DS, $0.1 \%$ epigallocatechin gallate-treated eyes show a significant decrease of corneal staining compared to the untreated group and vehicle-treated group, without differences between $0.01 \%$ epigallocatechin gallate-treated and vehicle-treated groups.

DED was induced in mice by administering scopolamine $(0.1 \mathrm{mg} / \mathrm{day})$ via implanted osmotic pumps and then exposing them to DS in a controlled-environment chamber $\left(23{ }^{\circ} \mathrm{C}, 20 \%\right.$ relative humidity, airflow from fans for $10 \mathrm{~h}$ a day). Cholinergic blockade with scopolamine and exposure to DS induce clinical disease through different primary pathogenic mechanisms. Scopolamine is a tropane alkaloid that antagonises muscarinic activity. Thus, it inhibits acetylcholine-mediated stimulation of the lacrimal gland by blocking muscarinic acetylcholine receptors. In the DED model, systemic administration of the anticholinergic scopolamine promotes pathogenic aqueous tear deficiency and inflammation of the lacrimal gland. ${ }^{370}$ In contrast, environmental stress induced by low relative humidity and constant airflow does not lead to significant tear deficiency. ${ }^{88}$ In this thesis, the results have shown that QCT and RES did not have any effect on tear production. Thus, topical administration of polyphenols did not affect lacrimal gland function. However, it has been recently shown that QCT is able to have an effect on the lacrimal gland, when it is administered orally in an in-vivo model of diabetes. ${ }^{371}$ The authors found that diabetic mice fed ad libitum for 3 months with an experimental diet containing $0.5 \%$ QCT have a significant recovery of tear volume, along with changes in the lacrimal gland morphology, which indicates improvements in the pathological features of diabetes. Based on these results, it is hypothesised that administration of polyphenols orally and topically might increase the anti-inflammatory effect on DED disease found in this thesis.

Cytokine/chemokine concentrations in tears were also analysed by immunobead assay: 6 and 4 out of 15 cytokines/chemokines included in the array were detected at days 6 and 10, respectively. This loss of cytokine/chemokine detection in the tear fluid at day 10 may be due in part to i) a concentration effect caused by decreased tear volume as the phenol red cotton thread test demonstrated, and/or ii) cytokine/chemokine levels increase at early stages of DS and decrease after 10 days of DS, as has been described previously for MMPs in tears of DS-exposed mice. ${ }^{372}$ Nevertheless, the pro-inflammatory cytokine IL-1 $\alpha$ and chemokine RANTES/CCL5 were increased in DS-exposed mouse tears. Topical treatment with $0.01 \%$ QCT, $0.1 \%$ RES or $0.01 \%$ QCT $+0.1 \%$ RES decreased IL-1a level. Although DS did not 
significantly increase IL-4 at day 6 and IP-10/CXCL10 at day 10 , there was a tendency to decreased cytokine/chemokine levels by $0.01 \%$ QCT. All of these molecular mediators can induce immune-mediated inflammation. For example, RANTES/CCL5 is a chemokine involved in recruitment of $T$ cells, IP-10/CXCL10 preferentially attracts Th1 cells, and IL-1a (a pro-inflammatory form of IL-1), is implicated in inducing inflammatory cytokine/chemokine expression (i.e. IL-6, IL-8/CXCL8, MCP-1/CCL2 and GM-CSF), as well as MMP synthesis by keratocytes, corneal fibroblasts and corneal epithelial cells. ${ }^{373}$ All of these pro-inflammatory mediators are increased in patients suffering DED. ${ }^{80,374}$ Thus, it is suggested that there is potential for QCT and RES as anti-inflammatory treatments of ocular surface diseases. In addition, Lee et al. demonstrated that polyphenols can also decrease inflammatory cytokine expression in mouse cornea. ${ }^{368}$ They quantified the transcripts encoding IL-1 $\beta$, TNF- $\alpha$ and MCP$1 / C C L 2$ in corneas of DS-exposed mice and found that treatment with topical $0.01 \%$ and $0.1 \%$ epigallocatechin gallate decreases the relative expression of IL-1 $\beta$ and MCP-1/CCL2 transcripts, but not the TNF- $\alpha$ transcript. These results suggest that the anti-inflammatory effect of topical polyphenols on cytokine secretion may occur, in part, at early stages of the inflammatory response. Thus, more studies are necessary to elucidate the mechanisms of how polyphenols inhibit cytokine/chemokine concentrations in tears of the inflamed ocular surface.

After 10 days of DS, an adoptive transfer experiment was performed to determine the effect of polyphenols in $\mathrm{CD} 4^{+} \mathrm{T}$ cells, in DS-induced ocular inflammation. As described in the "material and methods" section, draining CLN cells and spleen cells were collected from donor mice after 10 days of DS exposure and topical treatments with $0.01 \%$ QCT, $0.1 \%$ RES or the combination of both concentrations. CD4 ${ }^{+} \mathrm{T}$ cells were then adoptively transferred to recipient athymic (nude) mice, which were maintained under non-stressed conditions for $72 \mathrm{~h}$. The athymic mouse is an immunodeficient mouse that is not able to produce $\mathrm{CD} 4^{+} \mathrm{T}$ cells. Thus, the recipient mouse is a tabula rasa (blank slate) in that it is not exposed to anything that will initiate an innate response, giving a clean evaluation of the cellular adaptive response in the recipient. It should be noted that inflammation in recipients of CLN and spleen cells from DS donors is restricted to the LFU (cornea, conjunctiva and lacrimal gland), and no inflammatory cell infiltrates are found in the salivary gland, oral mucosa, thyroid, heart, lung, colon, spleen, adrenal gland or vagina. ${ }^{94}$ This model offers the advantage of more precisely dissecting T-cell-specific aspects (effector and regulatory) that contribute to the development of, and protection from, ocular surface autoimmunity. ${ }^{375}$ 
The results from this thesis have shown that recipients of DS-exposed donors had an increase of $\mathrm{CD} 4^{+} \mathrm{T}$ cell infiltration in the conjunctiva, although recipient mice were never exposed to DS. This is an expected result, as previously described by Niederkorn et al., who also found an increase of pro-inflammatory cytokine levels in tears, an extensive cellular infiltration into the conjunctiva, cornea and lacrimal gland, a decrease of TP and loss of goblet cells. ${ }^{94}$ These results described by Niederkorn and colleagues demonstrate that inflammation in a DS-induced mouse DED model is immune-mediated. Surprisingly, in our case, no decrease of TP and goblet cell numbers were observed, nor was there an increase of cytokine/chemokine concentration in tears of recipients after receiving CLN and spleen cells from DSexposed donors. Bearing in mind that this adoptive model lasted 3 days, a more prolonged adoptive transfer model might be required in order to find significant changes in aqueous tear production, goblet cell number and cytokine/chemokine concentration in tears of recipients.

The results from this thesis have also clarified that the anti-inflammatory effect of polyphenols is, in part, due to the inhibition of $\mathrm{CD}^{+} \mathrm{T}$ cells in recipient mice, which play an important role in the development of DED. ${ }^{70}$ The results have shown that 0.01 $\%$ QCT and $0.1 \%$ RES decreased $\mathrm{CD}^{+} \mathrm{T}$ cell infiltration in the conjunctiva of recipients of polyphenol-treated DS mice. In addition, the $0.01 \%$ QCT $+0.1 \%$ RES combination did not significantly decrease $\mathrm{CD}^{+} \mathrm{T}$ cell infiltration, but its value was clinically relevant because it was similar to that found in recipients of control mice. These results, which are likely to be the most important findings derived from this thesis, also corroborated the immunoregulatory effect of polyphenols on PBMCs invitro (described in Section VI.2).

Although more studies are needed to elucidate the mechanisms involved in the abolishment of the inflammatory response by polyphenols, it has been proposed that this inhibitory effect may also occur in cells involved with $\mathrm{CD} 4^{+} \mathrm{T}$ cell activation. For example, Lee et al. also studied the effect of epigallocatechin gallate on CD11 $\mathrm{b}^{+}$cells (monocytes/macrophages) in a DED model. ${ }^{368}$ They found that an increased number of central and peripheral corneal $\mathrm{CD} 11 \mathrm{~b}^{+}$cells in mice exposed to DS, whilst tropical treatment with $0.1 \%$ epigallocatechin gallate decreases $C D 11 b^{+}$cells, compared to the untreated and vehicle-treated groups. However, as the authors pointed out, the mere presence or absence of $\mathrm{CD}_{11 \mathrm{~b}^{+}}$cells may not reflect their functional status. APCs, such as monocytes/macrophages $\left(\mathrm{CD} 11 \mathrm{~b}^{+}\right)$and $\mathrm{DCs}\left(\mathrm{CD} 11 \mathrm{c}^{+}\right)$, play a pivotal role during the development and progression of DED by bridging the innate and adaptive immune response to activate autoreactive $T$ cells and maintain primed and targeted $T$ 
cells at the ocular surface..$^{70,99}$ In fact, one of the most recent studies by Dr. Stern and co-workers demonstrated that the APCs responses are modulated by Treg cells (inducible and natural), which increase early in response to DS and have an increased ability to suppress DC maturation and $\mathrm{T}$ cell proliferation by paracrine-mediated inhibition. ${ }^{376}$ Moreover, they also reported that stress-induced damage to the ocular surface resembling DED can occur independently of chronic $\mathrm{T}$ cell-mediated disease. ${ }^{377}$ Thus, further studies are necessary to elucidate whether QCT, RES and their combination have any effect on other immune cells that play a critical role in the in-vivo model of DED, such as APCs and Treg cells.

The anti-inflammatory therapies have been accepted as treatment of DED since numerous evidences showing the role of inflammation in the pathogenesis of DED. Therefore, the use of anti-inflammatory drugs may prove beneficial for both the subjective and objective complaints of DED. In this section, it has been demonstrated that both polyphenols, QCT and RES, decrease the clinical signs and inflammatory process underlying DS-induced DED, suppressing the local activation of CD4 ${ }^{+} \mathrm{T}$ cells in the conjunctiva of athymic mice. The anti-inflammatory effects of QCT and RES found in the two in-vitro models previously described, using ocular surface epithelial cells and $T$ cells, have been corroborated in the in-vivo model of DED. Moreover, polyphenols are able to protect the ocular surface against DS and scopolamine as CFS data showed. All together indicates that polyphenols act at different levels of the inflammatory response. These scientific evidences may establish QCT and RES as novel therapeutic agents for treating DED. 


\section{VII.4. In-vitro model of conjunctival mast cell response}

Mast cells are important effector cells in the conjunctiva because during allergic ocular diseases such as SAC, mast cells are activated following allergen cross-linking of surface $\mathrm{lgE}$ receptors, leading to degranulation and release of histamine, leukotrienes, prostaglandins and cytokines. In the present study, the effect of QCT, RES and their combinations were tested on CBMCs stimulated with $\lg \mathrm{E} / \mathrm{anti}-\mathrm{lgE}$.

One of the characteristics of an allergenic protein is that it induces specific $\lg E$ production and contains multiple $\lg E$ binding epitopes, so that the $\lg \mathrm{E}$ on mast cells can be cross-linked. As a result of IgE cross-linking, mast cells release their well-known inflammatory mediators and cytokines such as histamine and TNF- $\alpha$. The stimuli used to activate mast cells in-vitro is often not physiological and involves artificial chemicals such as compound 48/80, phorbol-12-myristate 13-acetate or calcium ionophore A23187. In the present study, IgE/Anti-IgE was used as a positive mast cell trigger for CBMCs. ${ }^{292}$ This model attempts to simulate the physiological stimulation by crosslinking of the FcERI (Figure 61). For this purpose, CBMCs are firstly sensitised with IgE. This sensitisation with $\operatorname{lgE}$ enhances FceRI expression on the surface of mast cells. Then, IgE-sensitised CBMCs are activated using anti-lgE, which potentially cross-links FcERI-bound IgE on mast cells. This event finally provokes mast cell degranulation.


Activation by $\lg E /$ Anti-lgE

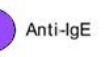

8.్టి. Pro-inflammatory mediators

Figure 61. Mechanisms of real and in-vitro mast cell activation by IgE cross-linking. Picture modified from Bax et al. ${ }^{378}$

In order to test the polyphenols on mast cells, activated CBMCs were treated with a wide range of QCT, RES or QCT+RES concentrations for $24 \mathrm{~h}$ to determine the toxic concentrations for CBMCs. Results showed that neither of QCT, nor RES, nor 
QCT+RES combinations decreased activated CBMC numbers. These results indicated that polyphenols are not toxic for CBMCs.

The anti-allergic effect of polyphenols was firstly tested on histamine release after $1 \mathrm{~h}$ of mast cell activation. IgE cross-linking with anti-IgE induced a significant increase of histamine. Surprisingly, the vehicle (EtOH) used to dissolve polyphenols decreased histamine secretion at both concentrations tested, $0.5 \%$ and $1 \%$. In fact, histamine levels found in the study of polyphenol combination was less than that found in control cells (unstimulated cells treated with EtOH).

In order to test the effects of QCT, RES and their combination on histamine release from CBMCs, dose-response curves were obtained for different concentrations of QCT and RES, and their combinations in two different experiments performed in single samples. After that, one combination of QCT and RES $(10 \mu \mathrm{M}$ QCT $+25 \mu \mathrm{M}$ RES) was chosen for studying its effect on the secretion of histamine by CBMCs, from one experiment performed in triplicate.

When CBMCs were treated with different concentrations of QCT, only $0.5 \mu \mathrm{M}$ QCT significantly decreased histamine release. Other QCT concentrations tended to decrease histamine levels, and all calculated $p$-values were close to the significance level, but did not reach statistical significance. Regarding RES treatments, only $5 \mu \mathrm{M}$ tended to decrease histamine secretion. Mean histamine values obtained from the remaining of RES concentrations were always lower, but not significant, than that obtained in stimulated cells treated with EtOH. Referring to the effect of QCT and RES combinations on histamine release, only 5 combinations out of 49 tested resulted in significantly decreased histamine levels. However, all of the mean values of histamine levels from each combination were also less than that found in stimulated cells treated with $\mathrm{EtOH}$. This fact may be due to two reasons: i) the effect of vehicle on histamine release (a low histamine value was found in stimulated cells treated with $\mathrm{EtOH}$, compared to unstimulated cells) and ii) the low number of samples (two experiments performed in single samples in dose-response curves). Therefore, increasing the number of samples may show significant differences, as found in the second experiment, in which $10 \mu \mathrm{M}$ QCT and $25 \mu \mathrm{M}$ RES alone decreased histamine levels significantly. This is a limitation commented on Section IX.

Previous studies have evidenced that QCT and RES have an inhibitory effect on histamine release. Kimata et al. tested the properties of QCT on human cultured mast cells stimulated with IgE/anti-lgE. ${ }^{379}$ They found that QCT (1 to $100 \mu \mathrm{M}$ ) shows a concentration-dependent inhibition of IgE-mediated histamine release and GM-CSF, leukotrienes and prostaglandin $D_{2}$ secretion by human mast cells. The authors also 
suggested that the target of QCT is not only FcERI-mediated activation, but also other intracellular events because QCT also inhibits histamine secretion when mast cells are exposed to calcium ionophore A23187 (a non-immunological stimulus for histamine release), indicating that $\mathrm{QCT}$ also regulates $\mathrm{Ca}^{2+}$ signalling pathway in mast cells. Regarding RES, Baolin et al. studied its effect on bone marrow-derived mouse mast cells. ${ }^{380}$ They reported that $100 \mu \mathrm{M}$ RES inhibits the release of histamine, leukotrienes and prostaglandin $D_{2}$ mediated by both $\lg E$ and calcium ionophore $A 23187$. These results suggested that RES also regulates $\mathrm{Ca}^{2+}$ signalling pathway in mast cells.

Degranulation of mast cells releases not only histamine, but also proinflammatory cytokines. Thus, the anti-allergic properties of polyphenols were also tested on the release of nine cytokines/chemokines: IL-1 $\beta$, IL-4, IL-5, IL-8/CXCL8, IL-9, IL-10, IL-13, TNF- $\alpha$ and IFN- $\gamma$. Firstly, dose-response curves for different concentrations of QCT and RES were obtained from two experiments performed in single samples. After that, the same combination of QCT and RES used on histamine release (10 $\mu \mathrm{M} \mathrm{QCT}+25 \mu \mathrm{M}$ RES) was tested on cytokine/chemokine secretion by CBMCs (one experiment performed in triplicate).

Cross-linking with anti-IgE induced a significant increase of IL-8/CXCL8, IL-13 and TNF- $\alpha$. On the other hand, IL-4, IL-5, IL-10 and IFN- $\gamma$ were detected but stimulation with $\lg \mathrm{E} / \mathrm{anti}-\mathrm{IgE}$ did not induce a significant increase of these cytokines. As found in the histamine assay, the final concentration of vehicle $(0.5 \% \mathrm{EtOH})$ produced a decrease of IL-8/CXCL8, IL-13 and TNF- $\alpha$ levels, but these levels were significantly elevated compared to unstimulated cells.

IL-1 $\beta$ and IL-9 were not detected at $24 \mathrm{~h}$, however it has been recently described by Mohd Zaki et al. that IL-9 is detected $24 \mathrm{~h}$ after anti-lgE stimulation of bone marrow-derived murine mouse mast cells, and reaches its maximum value at 48 h. ${ }^{381}$ The authors also reported that IL-9 positive cells are increased in patients suffering SAC and increased mast cell numbers co-localised with IL-9, indicating that these cells secrete IL-9. Thus, it is hypothesised that IL-9 may be detected at longer times, such as 48 or $72 \mathrm{~h}$ after IgE/anti-lgE stimulation of CBMCs.

Regarding the effect of different concentrations on the polyphenols QCT and RES, both decreased cytokine/chemokine levels of IgE/anti-lgE-stimulated CBMCs. QCT decreased IL-8/CXCL8 (10, 15 and $20 \mu \mathrm{M}), \mathrm{IL}-13(10,15$ and $20 \mu \mathrm{M})$ and TNF- $\alpha$ $(1,10,15,20$ and $25 \mu \mathrm{M})$. For RES treatments, these compounds also decreased IL8/CXCL8 $(10,25,50$ and $100 \mu \mathrm{M}), \mathrm{IL}-13(5,10,25$ and $100 \mu \mathrm{M})$ and TNF- $\alpha$ (1 to 100 $\mu \mathrm{M})$. These data showed that low concentrations of QCT and RES inhibited TNF- $\alpha$ secretion, whilst higher concentrations of polyphenols were necessary for decreasing 
IL-8/CXCL8 and IL-13 secretion. These data demonstrated that QCT and RES inhibit cytokine release from IgE/anti-lgE-stimulated CBMCs. Moreover, these results agree with previous studies, regarding the effect of both compounds on mast cells. For example, Min et al. investigated the effect of QCT on the expression of proinflammatory cytokines in a human mast cell line stimulated with phorbol-12-myristate 13-acetate and calcium ionophore A23187, and then treated with QCT. ${ }^{382}$ They found that QCT decreases the gene expression and production of TNF- $\alpha$, IL-1 $\beta$ and IL8/CXCL8 in stimulated cells. QCT also attenuates activation of NF-KB and p38 mitogen-activated protein kinase. In addition, Park et al. found that QCT inhibits gene expressions and secretion of the pro-inflammatory cytokines TNF- $\alpha$, IL-1 $\beta$ and IL8/CXCL8 on a human mast cell line, following stimulation with IgE or phorbol-12myristate 13-acetate and calcium ionophore A23187. ${ }^{383}$ Kempuraj et al. treated human CBMCs with QCT $(0.01,0.1,1,10$ or $100 \mu \mathrm{M})$ for $15 \mathrm{~min}$ before activation with anti$\operatorname{lgE}$. They found that the release of IL-8/CXCL8 and TNF- $\alpha$ is inhibited at $100 \mu \mathrm{M}$ QCT ${ }^{384}$ Regarding RES properties, Kang et al. demonstrated the effects of RES on the expression of pro-inflammatory cytokines in a human mast cell line. ${ }^{385}$ They stimulated cells with phorbol-12-myristate 13-acetate plus calcium ionophore A23187 in the presence of RES (10 and $50 \mu \mathrm{M}$ ) and found that RES significantly inhibits induction of the inflammatory cytokines IL-8/CXCL8 and TNF- $\alpha$. While Catalli et al. studied the effect of RES in comparison to the anti-allergy drug tranilast in both human mast cell line and human primary CD $34^{+}$-derived mast cells. They found that both RES and tranilast inhibit mast cell degranulation induced by substance $P$, IgE/anti-lgE and compound 48/80. RES inhibition is immediate, preventing degranulation when added simultaneously to the physiological stimuli, and the effect is sustained for up to $24 \mathrm{~h}$. RES also attenuates substance P-induced TNF- $\alpha$ and MCP-1/CCL2 production, and inhibits IgE-mediated release of leukotrienes, whilst tranilast is ineffective. Furthermore, RES reduces expression of the high-affinity IgE receptor, FcERI, on a human mast cell line. The effects of RES on mast cell activation is more marked in human primary CD $34^{+}$-derived mast cells, with this compound being significantly more efficacious than tranilast in these cells. ${ }^{386}$

Referring to the combination of QCT and RES, $10 \mu \mathrm{M}$ QCT + $25 \mu \mathrm{M}$ RES did not have any effect on cytokine/chemokine levels. However in this experiment, the levels of IL-8/CXCL8, IL-13 and TNF- $\alpha$ were not increased, and the cytokine/chemokine levels were not significantly reduced by QCT and RES alone, as expected from the dose-response data. Only the IL-5 level was significantly increased. It is hypothesised that the vehicle $(0.5 \% \mathrm{EtOH})$ had an inhibitory effect on the secretion of these mediators. Therefore, it was not been possible to determine the 
effect of both compounds together in this assay. In addition, above referenced authors dissolved RES and QCT with DMSO. In contrast to the results found in this thesis, they did not report any effect of this solvent on cytokine release and mast cell degranulation. Thus, DMSO may be better solvent for testing polyphenols on mast cells than $\mathrm{EtOH}$. However, DMSO was discarded as a solvent for all in-vitro experiments due to its toxic effect on cells. Nevertheless, it has been suggested that combinations of polyphenols can have anti-allergic effects in-vitro and in-vivo. For example, Cruz et al. reported on the protective effect of Kalanchoe pinnata (a perennial plant found in tropical areas) extract in fatal anaphylactic shock, likewise a Th2-driven immunopathology. ${ }^{387}$ They demonstrated that mice treated daily with oral Kalanchoe pinnata during hypersensitisation with ovalbumin are all protected against death when challenged with the allergen, as compared with the $100 \%$ mortality rate observed in the untreated group. Oral protection is accompanied by a reduced production of ovalbumin-specific $\mathrm{IgE}$ antibodies, reduced eosinophilia and impaired production of the IL-5, IL-10 and TNF- $\alpha$ cytokines. They also found that Kalanchoe pinnata prevented antigen-induced mast cell degranulation and histamine release in-vitro. ${ }^{387}$ The authors did not find any inhibitory effect of the vehicle (PBS) on mast cells degranulation. Polyphenols used in these experiments from Kalanchoe pinnata were soluble in water-based solution because authors extracted polyphenols with distilled water.

Mast cells are one type of cells that take part in the acute allergic response owing to their release of synthesised and stored inflammatory mediators, such as histamine, cytokines and proteases. Thus, their activation plays an important role in the pathogenic process of ocular allergy. Activation of mast cells with IgE/anti-lgE results in the secretion of the preformed contents of their granules, such as histamine and cytokines. This thesis has shown that QCT and RES inhibit the release of histamine and cytokines mediated by IgE/anti-lgE activation of CBMCs. This indicated that both compounds are effective against IgE receptor-mediated mast cell degranulation. Thus, this study demonstrates that polyphenols differentially modulate two important effector functions, histamine release and cytokine expression of $\lg E / a n t i-l g E$ activated mast cells. However, the molecular targets of polyphenols on IgE-mediated allergy pathway need to be determined in further experiments. 



\section{Summary of thesis results}



The aim of this thesis was to corroborate the following hypothesis:

\section{"The topical application of QCT, RES and their combination has an anti- inflammatory effect on ocular surface diseases such as dry eye and ocular allergy, which is due to modulation of epithelial cells and immune cell responses"}

The most important results from this thesis are shown in Figures 62 and 63, which summarise results from all of the in-vitro models and in-vivo model, respectively. QCT, RES and their combination have shown to target several inflammatory mediators/pathways that have been identified in ocular surface diseases, such as DED and ocular allergy.

In order to test the potential effect of QCT, RES and their combination, compounds were tested on three in-vitro models of inflammation and allergy, and one in-vivo model of DED. Firstly, polyphenols were tested on two ocular surface (conjunctival and corneal) epithelial cell lines stimulated with the cytokine TNF- $\alpha$ and UV-B light exposure by measuring cytokine secretion, COX-2 expression and intracellular ROS production. After that, the effect of polyphenols on $\mathrm{T}$ cell activation and proliferation was studied on anti-CD3/anti-CD28-stimulated PBMCs. Thirdly, the anti-inflammatory effects of topically-applied polyphenols were tested in a murine model of DED, followed by an adoptive transfer model. CFS, TP, goblet cell number, cytokine/chemokine levels in tears and $\mathrm{CD}^{+} \mathrm{T}$ cell infiltration in conjunctiva were measured. Finally, the anti-allergic effects of polyphenols were studied on CBMCs stimulated by $\lg \mathrm{E}$ cross-linking with anti-lgE. Histamine secretion and cytokine/chemokine release were determined.

Regarding the in-vitro models, experiments performed on ocular surface (conjunctival and corneal) epithelial cells demonstrated that QCT, RES and their combination caused inhibition of TNF- $\alpha$-stimulated cytokine/chemokine secretion (IL-6, IL-8/CXCL8 and IP-10/CXCL10) and COX-2 expression, as well as decreased intracellular ROS production in UV-B-exposed cells. The immunoregulatory effect of QCT+RES was demonstrated on anti-CD3/anti-CD28-stimulated PBMCs by inhibiting cell proliferation. Moreover, the anti-allergic properties of QCT, RES and QCT+RES were finally demonstrated on IgE/anti-IgE-stimulated CBMCs by decreasing histamine levels and cytokine/chemokine secretion (IL-8/CXCL8, IL-13 and TNF- $\alpha$ ), after mast cell degranulation (Figure 62). 


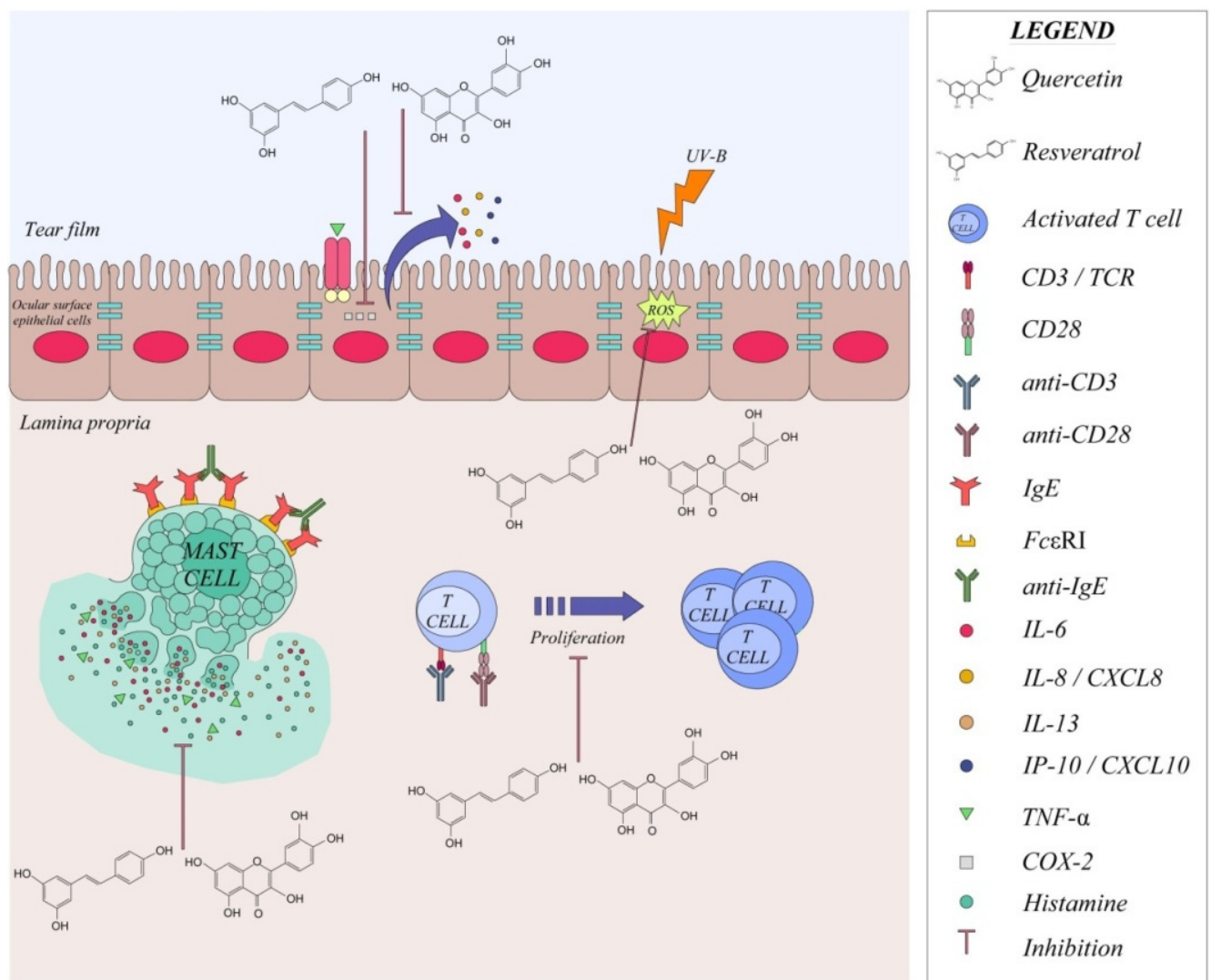

Figure 62. Summary of the most important results from in-vitro experiments.

Regarding the in-vivo model of DED, results from this thesis have shown that $\mathrm{QCT}, \mathrm{RES}$ and $\mathrm{QCT}+\mathrm{RES}$ protect the ocular surface from DS exposure. In addition, $\mathrm{QCT}, \mathrm{RES}$ and $\mathrm{QCT}+\mathrm{RES}$ reduce the infiltration of $\mathrm{CD} 4^{+} \mathrm{T}$ cells in the conjunctiva of athymic mice (Figure 63).



Figure 63. Summary of the most important results from in-vivo experiments. CFS: corneal fluorescein staining. Adapted from Stern et al. 2013. ${ }^{70}$ 
QCT and RES have demonstrated their anti-inflammatory properties in different types of cells that are involved in several ocular surface diseases, and were corroborated in an animal model of DED. Consequently, the topical application of QCT, RES or their combination might be promising anti-inflammatory therapy for ocular surface diseases such as DED and ocular allergy. Moreover, they may have the potential to help improve UV-B exposure-induced ocular surface disorders, because current clinical therapy for ocular surface inflammation does not offer any protection against UV-B radiation. ${ }^{388}$

Summary of results of this thesis:

1. Quercetin, resveratrol and their combination have an anti-inflammatory effect on ocular surface epithelial cells by inhibiting cytokine/chemokine secretion and cOX-2 expression in an in-vitro model of ocular inflammation.

2. Quercetin, resveratrol and their combination have an antioxidant effect on ocular surface epithelial cells by decreasing intracellular reactive oxygen species production in-vitro in cells exposed to ultraviolet light.

3. Quercetin and resveratrol combination have an immunoregulatory effect on $T$ cells by inhibiting the activation and proliferation of human peripheral blood mononuclear cells in-vitro.

4. Quercetin, resveratrol and their combination have anti-allergic effects on mast cell degranulation by inhibiting histamine release and cytokine/chemokine secretion in-vitro.

5. Topical application of quercetin, resveratrol and their combination decreases ocular signs and cytokine levels in tears of mice in an in-vivo model of DED.

6. Topical application of quercetin and resveratrol avoid $\mathrm{CD} 4^{+} \mathrm{T}$ cell infiltration in conjunctiva of mice in an adoptive transfer model. 



\section{Limitations and future studies}



This work has some limitations that are addressed in this section.

The anti-inflammatory and antioxidant effects of polyphenols were firstly tested in two ocular surface cell lines (conjunctival -IOBA-NHC-and corneal -HCE- epithelial cells) stimulated by TNF-a. Both cell lines are commonly used to study physiological and pathophysiological mechanisms involved in inflammation, and also to test new potential compounds for treating ocular surface diseases. The cellular responses in cell lines do not reflect the complexity of the human ocular surface epithelia. However, invitro models offer several advantages, such as the study of the underlying biological mechanisms of action of drugs and they allow the testing of several drugs without a large financial investment. Another significant limitation is the fact that these tests were performed using a monolayer rather than a stratified structure, as is found in the ocular surface. Finally, the anti-inflammatory effect of polyphenols has only been tested using the pro-inflammatory cytokine TNF- $\alpha$ (which did not stimulate COX-2 secretion by HCE cells), and inflammatory pathways involved in ocular surface inflammation (such as MAPK) have not been studied in these in-vitro models. Thus, it is proposed for further studies in ocular surface epithelial cells:

i) to test the anti-inflammatory effect of polyphenols in stratified cultures of human epithelial cells;

ii) to use other pro-inflammatory cytokines involved in moderate-severe ocular surface inflammation for stimulating cells, i.e. IL-4, IL-13, IFN-y and/or TGF- $\beta$; and

iii) to study cellular pathways involved in ocular surface inflammation.

In addition to that, the antioxidant effect of polyphenols was studied using UV-B radiation. For this purpose, only intracellular ROS was measured. However, it is well known that ROS are involved in apoptosis. Moreover, UV-B radiation is one of the most common inductors of ocular surface epithelial apoptosis. Thus, future studies might address the effect of both polyphenols in ocular surface epithelial cell apoptosis. Regarding this point, a preliminary study was performed during the stay in Allergan, looking at the effect of polyphenols in the mitochondrial permeability transition pore, using IOBA-NHC cells. These preliminary results have been included in Appendix II.

After testing the anti-inflammatory effects of polyphenols in conjunctival and corneal epithelial cells, the immunomodulatory properties of QCT, RES and QCT+RES were studied in PBMCs isolated from healthy donors, analysing viability and proliferation of PBMCs treated with polyphenols. This part of the thesis also has some limitations. For example, PBMCs used in this study were only obtained from three subjects. In addition, experiments from this thesis have demonstrated the antiproliferative effect of QCT+RES, but not for both compounds alone at the 
concentrations tested. Moreover, there was a lack of studies on the molecular mechanisms underlying their antiproliferative effects. In addition, PBMCs are a mix of different cell subsets that may be affected by polyphenols in different ways. Therefore, further studies may include:

i) experiments with a larger population;

ii) to test more concentrations of QCT and RES individually;

iii) assays for studying molecular mechanisms involved in inflammation; and

iv) characterisation of the modulatory effect of polyphenols on the different subsets of $T$ cells.

The anti-inflammatory effects of QCT, RES and QCT+RES were studied in a murine model of DED, followed by an adoptive transfer model. For this purpose, 0.01 $\%$ QCT and $0.1 \%$ RES and their combination were tested. There were some limitations regarding these experiments. Firstly, $\mathrm{CD}^{+} \mathrm{T}$ cell infiltration in conjunctiva was studied by immunohistochemistry, but this cell marker is expressed by several cell types and it is well known that the different subtypes of $\mathrm{CD} 4^{+} \mathrm{T}$ cells (i.e. Th1, Th2, Th17 and Treg cells) are involved in the development of experimental DED model. Therefore, future studies might evaluate the effect of polyphenols on CD4 ${ }^{+} \mathrm{T}$ cell subtypes. Secondly, APCs play an important role in the development of DED being necessary for the initiation and development of the disease. Moreover, a previous study supports the idea that polyphenols may act on $C D 11 b^{+} D C s$. Therefore, it would be very interesting to look into the effect of QCT, RES and their combination on APCs. In addition, it is also well known that oxidative stress is involved in DED because increase markers of DNA oxidative damage (i.e. 8-OHdG), and lipid peroxidation markers (such as 4-hydroxy-2-nonenal and MDA) are found in a mouse model of DED. ${ }^{389}$ Thus, the antioxidant effect of both polyphenols on experimental DED may be tested as their antioxidant effects have been demonstrated in ocular surface epithelial cells in this thesis. All of these future experiments on cells of the immune system would allow characterisation of the mechanisms underlying polyphenols as anti-inflammatory compounds in DED. Finally, only two concentrations of QCT and RES, and their combination were tested. In-vitro results demonstrated that the mixed low concentrations of both polyphenols proved the anti-inflammatory activity of QCT and RES, compared to those results found in cells treated with polyphenols separately. Thus, future studies could evaluate the effect of low concentrations of QCT, RES and their combinations in this in-vivo model of DED. 
The anti-allergic properties of polyphenols QCT and RES on CBMCs have also been demonstrated in this thesis, but with some limitations. Firstly, polyphenols have been demonstrated to have inhibitory effects on histamine and cytokine release in response to $\lg \mathrm{E}$ cross-linking. Yet other mediators, such as leukotrienes and prostaglandin $D_{2}$, have not been studied, as well as the intracellular mechanism underlying the effects of both polyphenols. Secondly, this is an in-vitro model using mast cells, which play a key role in ocular allergic diseases, but there are other cells that are involved in these disorders such as neutrophils, basophils and eosinophils. Another limitation of this study was the fact that the vehicle $(\mathrm{EtOH})$ used for dissolving polyphenols had an effect on CBMCs degranulation at low doses. Lastly, the in-vitro model does not reflect the complexity of the allergic response to the human ocular surface. Therefore, further studies should include:

i) another vehicle to avoid artefacts (i.e. DMSO);

ii) experiments for studying the effect of polyphenols and their combinations on leukotrienes and prostaglandin $\mathrm{D}_{2}$ and their molecular mechanisms;

iii) assays for testing the polyphenols effects on other cell types involved in allergy; and iv) an in-vivo model of ocular allergy as an approximation to the response in the ocular allergic process.

This thesis has demonstrated the anti-inflammatory effect of QCT, RES and their combination on ocular surface epithelial cells, T cells, mast cells and in a murine model of DED. The main target of any research is to bring discoveries from the bench to the bedside. This purpose inspires the target of IOBA: "investigar para curar mejor" (to research to heal better). Therefore, the most important future study would be to test the effect of both QCT and RES topically in patients suffering DED. For this aim, experiments could be performed in a controlled environmental chamber (CER-lab) located in the IOBA building, in which pressure, temperature and relative humidity can be controlled. This future study could provide clinical evidence that QCT and RES may be useful in the treatment of DED. 



\section{Conclusions}



1. Quercetin and resveratrol are naturally-occurring compounds with antiinflammatory and antioxidant properties on human epithelial cells from the cornea and conjunctiva, and immunoregulatory effects preventing activation of cells of the immune system, such as lymphocytes and mast cells.

2. Both compounds have shown these properties separately and in combination, indicating the necessity for future experiments to determine what concentrations of each individually, or both in combination, are most effective to use.

3. Their properties have shown that these compounds could be used as topical treatment of inflammatory diseases of the ocular surface, such as dry eye or ocular allergy, as results from the murine model of dry eye have shown. 

XI. Resumen en español 



\section{Objetivos}

Los objetivos generales y específicos planteados para comprobar la hipótesis establecida en esta tesis son:

\section{Objetivo general:}

Estudiar el efecto de los compuestos quercitina, resveratrol y su combinación en los procesos inflamatorios de la superficie ocular con base inmune como el ojo seco y la alergia ocular.

\section{Objetivos específicos:}

- Determinar el efecto anti-inflamatorio de quercitina, resveratrol y su combinación en dos líneas celulares procedentes de los epitelios conjuntival y corneal humano estimuladas con TNF- $\alpha$.

- Determinar el efecto antioxidante de quercitina, resveratrol y su combinación en dos líneas celulares procedentes de los epitelios conjuntival y corneal humano irradiadas con luz UV-B.

- Determinar la actividad inmunorreguladora de quercitina, resveratrol y su combinación en células mononucleares procedentes de sangre periférica humana.

- Determinar el efecto terapéutico de la aplicación tópica de de quercitina, resveratrol y su combinación en un modelo murino de ojo seco.

- Determinar el efecto de de quercitina, resveratrol y su combinación en la degranulación de mastocitos. 


\section{Material y métodos}

\subsection{Modelo in-vitro de inflamación y estrés oxidativo en células epiteliales conjuntivales y corneales humanas}

El efecto anti-inflamatorio y antioxidante de QCT, RES y su combinación fue estudiado in-vitro en dos líneas celulares, la primera derivada del epitelio conjunctival humano (IOBA-NHC) y la segunda derivada del epitelio corneal humano (HCE), utilizando dos estímulos diferentes: 1) inflamación inducida por TNF- $\alpha$ y 2) estrés oxidativo provocado por luz UV.

La citotoxicidad de los polifenoles fue estudiada en primer lugar para determinar las concentraciones no tóxicas para ambas líneas celulares. Después, el efecto de QCT, RES y su combinación fue estudiado en la secreción de citoquinas y quimioquinas, la expresión de COX-2 y la producción de ROS.

\subsubsection{Reactivos}

Todos los reactivos y anticuerpos (tabla 1) usados en esta parte de la tesis han sido agrupados según el fabricante:

- Invitrogen (Inchinnan, Reino Unido): Dulbecco's Modified Eagle Medium/Nutrient Mixture F12 (DMEM/F12), test alamarBlue ${ }^{\circledR}$ para la viabilidad celular, ácido 4-(2hidroxietil)-1-piperazineetanosulfónico (HEPES).

- Nunc (Roskilde, Dinamarca): Frascos y placas de cultivo celular.

- Panreac (Barcelona, España): EtOH y D-glucosa.

- PeproTech EC (Londres, Reino Unido): TNF-a.

- Sigma-Aldrich (St Louis, MO, EE.UU.): RES, QCT, DMEM (medio de cultivo sin $\mathrm{NaHCO}_{3}$, piruvato sódico y rojo fenol), diacetato de 2',7'-diclorodihidrofluoresceína $\left(\mathrm{H}_{2} \mathrm{DCF}-\mathrm{DA}\right)$, L-glutamina, suero fetal bobino (FBS), toxina colérica, factor de crecimiento epitelial humano (EGF), insulina bobina, penicilina, estreptomicina, fungizona, hidrocortisona, dimetil sulfóxido (DMSO), tampón fosfato salino (PBS), tris-ácido clorhídirico (Tris- $\mathrm{HCl})$, cloruro sódico $(\mathrm{NaCl})$, ácido desoxicólico, Triton X100, dodecil sulfato de sodio (SDS), ácido etilendiaminotetraacético (EDTA), fluoruro de fenilmetilsulfonilo (PMSF), aprotinina, ortovanadato sódico $\left(\mathrm{Na}_{3} \mathrm{VO}_{4}\right)$, glicerol, 2-mercaptoetanol (2-ME), azul de bromofenol, albumina de suero bovino (BSA), Tween 20, tris-tampón salino (TBS). 
- Thermo Fisher Scientific (Rockford, IL, EE.UU.): ácido bicinconínico (BCA).

\begin{tabular}{|c|c|c|c|}
\hline Anticuerpo & Dilución & Fabricante & Referencia \\
\hline $\begin{array}{c}\text { Conejo anti-COX-2 } \\
\text { fosfato deshidrogenasa } \\
\text { (GAPDH) }\end{array}$ & $1 / 100$ & $\begin{array}{c}\text { Novus Biologicals } \\
\text { (Littleton, CO, USA) }\end{array}$ & NB100-689 \\
\hline $\begin{array}{c}\text { HRP-conjugado humano } \\
\text { anti-lgG }\end{array}$ & $1 / 500$ & $\begin{array}{c}\text { Santa Cruz Biotechnology } \\
\text { (Heidelberg, Germany) }\end{array}$ & SC-166545 \\
\cline { 1 - 2 } $\begin{array}{c}\text { HRP-conjugado ratón anti- } \\
\text { IgG }\end{array}$ & $1 / 5000$ & $\begin{array}{c}\text { Jackson Laboratory (Bar } \\
\text { Harbor, ME, USA) }\end{array}$ & SC-2004 \\
\hline
\end{tabular}

Tabla 1. Anticuerpos utilizados en esta parte de la tesis.

\subsubsection{Preparación de las disoluciones de los polifenoles}

QCT y RES fueron disueltos en EtOH. Cada disolución de QCT y RES se preparó antes de cada experimento por dilución seriada a partir de disoluciones madre recién preparadas. Las concentraciones finales en cada experimento fueron 0,5-25 $\mu \mathrm{M}$ de QCT y 0,5-300 $\mu \mathrm{M}$ de RES.

La disoluciones en las cuales se combinaron QCT y RES fueron preparadas mezclando disoluciones individuales de QCT y RES para conseguir las concentraciones finales deseadas para cada experimento.

Todas las disoluciones fueron preparadas para conseguir una concentración final no tóxica del vehículo $(\mathrm{EtOH})$ del $0,5 \%$ en todas las muestras tratadas con los polifenoles. Las disoluciones se mantuvieron en todo momento en oscuridad para evitar su degradación por la luz solar.

\subsubsection{Líneas celulares y condiciones de cultivo}

Se utilizaron dos líneas celulares procedentes del epitelio de la superficie ocular, IOBA-NHC y HCE, para los experimentos de esta parte de la tesis.

A) La línea celular IOBA-NHC es una línea inmortalizada espontáneamente, no transfectada, procedente de la conjuntiva humana normal. Las células IOBA-NHC se cultivaron en medio DMEM/F-12 L-glutamina suplementado con $10 \%$ de FBS, 0,1 $\mu \mathrm{g} / \mathrm{mL}$ de toxina colérica, $2 \mathrm{ng} / \mathrm{mL}$ EGF, $1 \mu \mathrm{g} / \mathrm{mL}$ de insulina bovina, $5000 \mathrm{U} / \mathrm{mL}$ de 
penicilina, $5 \mathrm{mg} / \mathrm{mL}$ de estreptomicina, $2,5 \mu \mathrm{g} / \mathrm{mL}$ de fungizona y $0,5 \mu \mathrm{g} / \mathrm{mL}$ de hidrocortisona.

B) La línea celular HCE es una línea inmortalizada procedente del epitelio corneal humano donada por el Profesor Arto Urti (Universidad de Helsinki, Finlandia). Las células HCE se cultivaron en medio DMEM/F-12 L-glutamina suplementado con $15 \%$ de FBS, 0,5 \% DMSO, 0,1 $\mu \mathrm{g} / \mathrm{mL}$ toxina colérica, $10 \mathrm{ng} / \mathrm{mL}$ de EGF, $5 \mu \mathrm{g} / \mathrm{mL}$ de insulina, $100 \mathrm{U} / \mathrm{mL}$ de penicilina y $0,1 \mathrm{mg} / \mathrm{mL}$ de estreptomicina.

Todos los experimentos se llevaron a cabo utilizando un medio de cultivo DMEM sin $\mathrm{NaHCO}_{3}$, piruvato sódico y rojo fenol y suplementado con $3.15 \mathrm{~g} / \mathrm{L}$ de Dglucosa, $2 \mathrm{mM}$ de L-glutamina y $25 \mathrm{mM}$ de HEPES. Se hará referencia a este medio como medio completo (CM) a lo largo de todo el texto.

\subsubsection{Ensayo de citotoxicidad}

La citotoxicidad de QCT, RES y QCT+RES en las células epiteliales se determinó por la reducción del reactivo resazurina (alamarBlue ${ }^{\circledR}$ ). Para realizar los ensayos, las células IOBA-NHC y HCE se plantaron en placas de 96 pocillos y se dejaron crecer durante 3 días. Después, el medio de cultivo se sustituyó por medio puro, sin suero y sin suplementos, en el cual se mantuvieron las células durante $24 \mathrm{~h}$. Entonces, el medio puro se reemplazó por $\mathrm{CM}$ y las células se trataron con diferentes concentraciones de QCT, RES o QCT+RES y se incubaron durante $24 \mathrm{~h}$ a $37{ }^{\circ} \mathrm{C}$. Las células control fueron tratadas con el vehículo $(0,5 \%$ EtOH). Después de las $24 \mathrm{~h}$ de incubación, se eliminaron los sobrenadantes y se añadió a cada muestra una disolución de alamarBlue ${ }^{\circledR}$ al $10 \%$, preparada en el medio de cultivo suplementado DMEM/F12. Las células se incubaron durante $4 \mathrm{~h}$ a $37^{\circ} \mathrm{C}$. Finalmente, el medio de cada muestra fue recogido y se midió la fluorescencia de cada uno de ellos en un espectrofotómetro UV/Vis (SpectraMax ${ }^{\circledR}$ M5, Molecular Devices, Sunnyvale, CA, EE.UU.).

\subsubsection{Estimulación celular con TNF- $\alpha$ y tratamiento con los polifenoles}

Para este experimento, las células IOBA-NHC and HCE se plantaron en placas de 24 pocillos y se dejaron crecer durante 3 días. Para el ensayo de la expresión de COX-2, las células se plantaron en frascos de cultivo de $25 \mathrm{~cm}^{2}$ hasta confluencia. Pasado el tiempo de incubación, el medio de cultivo se reemplazó por medio de cultivo 
puro, sin suero ni suplementos, y las células se mantuvieron en él durante $24 \mathrm{~h}$ a 37 ${ }^{\circ} \mathrm{C}$. Después de las $24 \mathrm{~h}$ de incubación, el medio puro se reemplazó por medio CM y las células fueron pretratadas con QCT, RES, QCT+RES o vehículo durante $2 \mathrm{~h}$ a 37 ${ }^{\circ} \mathrm{C}$. Después, se quitaron los pretratamientos, las células se estimularon con $25 \mathrm{ng} / \mathrm{mL}$ de TNF- $\alpha$ en presencia de QCT, RES, QCT+RES o vehículo en medio CM y se dejaron en incubación durante $24 \mathrm{~h}$. Se utilizaron como controles células no estimuladas con TNF- $\alpha$ pero tratadas con los polifenoles. Después de las $24 \mathrm{~h}$, el medio de cada muestra se recogió y se centrifugó a 59 x g durante $5 \mathrm{~min}$. Las placas y los frascos con las células adheridas y los sobrenadantes se guardaron a $-80^{\circ} \mathrm{C}$ antes de ser analizados.

\subsubsection{Lisis celular y determinación de la proteína total}

El contenido total de proteína fue determinado tanto en los frascos como en las placas con las células adheridas. En primer lugar, las células fueron lisadas con buffer de lisis (RIPA) [10 mM tris- $\mathrm{HCl}(\mathrm{pH} 7,4), 150 \mathrm{mM}$ de $\mathrm{NaCl}, 1 \%$ de ácido desoxicórico, $1 \%$ de Triton $\mathrm{X}-100,0,1 \%$ de SDS y $1 \mathrm{mM}$ de EDTA] suplementado con $0,1 \mathrm{mg} / \mathrm{mL}$ de PMSF, $60 \mu \mathrm{g} / \mathrm{mL}$ de aprotinina y $0,1 \mu \mathrm{M}$ de $\mathrm{Na}_{3} \mathrm{VO}_{4}$. Las muestras se incubaron en hielo durante 30 min y finalmente se centrifugaron y se almacenaron a $-80^{\circ} \mathrm{C}$ antes de ser usadas.

La cantidad de proteína total fue determinada con el test BCA. Brevemente, 25 $\mu \mathrm{L}$ de las muestras estándar y de los lisados celulares fueron tratados con el reactivo de trabajo BCA (BCA en 0,1 mM hidróxido sódico y $4 \%$ de una solución de sulfato de cobre (II)) e incubados durante $30 \mathrm{~min}$ a $37^{\circ} \mathrm{C}$. Entonces, las muestras se enfriaron a temperatura ambiente y se midió la absorbancia de cada muestra a $562 \mathrm{~nm}$ utilizando el espectrofotómetro SpectraMax ${ }^{\circledR}$ M5 UV/Vis (Molecular Devices). Los datos fueron analizados con el software SoftMax ${ }^{\circledR}$ Pro (Molecular Devices).

\subsubsection{Medida de la secreción de citoquinas y quimioquinas}

La secreción de citoquinas y quimioquinas fue medida de manera simultánea con un ensayo multianalito utilizando tecnología $x-M A P^{\circledR}$. Los niveles de IL-6, IL8/CXCL8, IP-10/CXCL10 y VEGF fueron determinados en los sobrenadantes celulares. Brevemente, $25 \mu \mathrm{L}$ de cada muestra se cultivaron con las respectivas microesferas o "beads" a $4{ }^{\circ} \mathrm{C}$ durante toda la noche. Después, se incubaron con el anticuerpo biotinilado durante $1 \mathrm{~h}$ a temperatura ambiente seguida de otra incubación 
con estreptavidina-ficoeritrina durante $30 \mathrm{~min}$. Finalmente, las microesferas se analizaron con un Luminex ${ }^{\mathrm{TM}}$ 100-IS (Luminex Corporation, Austin, Tx, EE.UU.) y con el software BeadView ${ }^{\mathrm{TM}}$ Software (Upstate, Reino Unido).

\subsubsection{Expresión de ciclo-oxigenasa-2}

La expresión de COX-2 en las células epiteliales se determinó mediante gel de poliacrilamida SDS-PAGE y Western blotting. El homogeneizado celular se mezcló con 2X Laemmli [4 \% de SDS, $20 \%$ de glicerol, $10 \%$ de 2-ME, 0,004 \% de azul de bromofenol y 0,125 mM de Tris- $\mathrm{HCl}, \mathrm{pH} 6,8]$.

Las proteínas en las muestras se desnaturalizaron por calor a $110^{\circ} \mathrm{C}$ durante 5 min. Entonces, $20 \mu \mathrm{g}$ de proteína total se separaron con un $10 \%$ de gel SDS-PAGE. Después, se transfirieron a una membrana porosa donde se bloquearon con $5 \%$ de leche desnatada, $3 \%$ de BSA y 0,05 \% de Tween 20 en TBS durante 1 h. La membrana se incubó con anticuerpo para COX-2 toda la noche a $4{ }^{\circ} \mathrm{C}$. Seguidamente, se incubaron con anticuerpo para IgG durante $1 \mathrm{~h}$ a temperatura ambiente. Finamente, se detectaron las bandas inmunorreactivas de COX-2 con solución quimioluminiscente (Santa Cruz) durante $1 \mathrm{~min}$. Las imágenes se capturaron con la cámara CCD incorporada en un ChemiDoc XRS (Bio-Rad, Inc., Hercules, CA, EE.UU.). La banda de GAPDH se utilizó como control para normalizar los niveles de COX-2.

\subsubsection{Medida de especies reactivas de oxígeno inducidas por luz UV-B}

La generación intracelular de ROS por radiación UV-B se determinó con el compuesto $\mathrm{H}_{2}$ DCF-DA. Las células IOBA-NHC and HCE se plantaron y cultivaron en placas de 24 pocillos transparentes para la luz UV-B. Una vez eliminados los medios de cultivo y mantenidas las células en medio puro durante $24 \mathrm{~h}$, se pretrataron las células con QCT, RES, QCT+RES o vehículo durante $1 \mathrm{~h}$ a $37{ }^{\circ} \mathrm{C}$. Después, se eliminaron los tratamientos y se cargaron las células con una disolución $10 \mu \mathrm{M}$ de $\mathrm{H}_{2}$ DCF-DA en medio $\mathrm{CM}$ durante $30 \mathrm{~min}$. Entonces, se eliminó la disolución de $\mathrm{H}_{2}$ DCF-DA, se volvieron a tratar las células con los polifenoles y se irradiaron con una lámpara UV-B de $8 \mathrm{~W}$ con el pico máximo a $302 \mathrm{~nm}$ durante $15 \mathrm{~s}$. La energía final que recibieron las células fue de $107,25 \mathrm{~mJ} / \mathrm{cm}^{2}$. Las células control no se irradiaron. Seguidamente, las células se dejaron en incubación durante $1 \mathrm{~h}$ y finalmente se determinó la fluorescencia con un espectrofotómetro SpectraMax ${ }^{\circledR}$ M5 UV/Vis (Molecular Devices). 


\subsection{Modelo in-vitro de proliferación de linfocitos $\mathbf{T}$}

El efecto inmunosupresor de los compuestos QCT, RES y su combinación se estudió en células procedentes de sangre periférica humana (PBMCs). Se determinó la citotoxicidad, y la proliferación y viabilidad.

\subsubsection{Reactivos}

Todos los reactivos se adquirieron en Sigma-Aldrich si no se especifica otra cosa. Todos los ensayos se realizaron con el medio de cultivo modificado Roswell Park Memorial Institute (RPMI)-1640 HEPES suplementado con 2 mM de L-glutamina, $10 \%$ de FBS, $50 \mathrm{U} / \mathrm{mL}$ de penicilina, 50 de $\mu \mathrm{g} / \mathrm{mL}$ de estreptomicina, aminoácidos no esenciales, $1 \mathrm{mM}$ de piruvato sódico y $2,5 \mu \mathrm{M}$ de 2-ME. El ioduro de propidio (PI) también se adquirió en Sigma.

Los anticuerpos anti-CD3 (clon HIT3a) y anti-CD28 (clon 28.2) se adquirieron en Becton Dickinson Biosciences (BD, Oxford, Reino Unido). El succinimidil éster de diacetato de carboxifluoresceína (CFSE) y Ficoll-Hypaque se adquirieron en Invitrogen. El suero fetal bovino (FCS) se adquirió en Labtech International (Ringmer, Reino Unido).

\subsubsection{Preparación de las disoluciones de polifenoles}

Las disoluciones de QCT y RES se prepararon a partir de disoluciones madre en EtOH por dilución. Las concentraciones finales fueron de 0,5-25 $\mu \mathrm{M}$ de QCT y 0,5$100 \mu \mathrm{M}$ de RES. La concentración del vehículo para los tratamientos individuales de los polifenoles fue siempre de $0,5 \%$ de $\mathrm{EtOH}$.

Las concentraciones finales de QCT+RES se consiguieron añadiendo cada disolución de QCT y RES a cada muestra, de esta manera la concentración final de vehículo fue siempre el $1 \%$ de $\mathrm{EtOH}$.

\subsubsection{Donantes}

La sangre periférica se obtuvo de tres pacientes anónimos que leyeron y firmaron el consentimiento informado. Los protocolos utilizados fueron revisados y aprobados por los comités éticos de la UCL y de la Universidad de Valladolid. 


\subsubsection{Aislamiento de las células mononucleares}

Las células PBMC se aislaron mediante gradiente de densidad con FicollHypaque. La sangre se diluyó en medio de cultivo y se añadió lentamente sobre el Ficoll para luego centrifugar la muestra a $624 \times \mathrm{g}$ durante $40 \mathrm{~min}$. Las células mononucleares se recogieron, se lavaron, se volvieron a centrifugar y, finalmente, se resuspendieron en medio de cultivo para su uso.

\subsubsection{Ensayo de citotoxicidad}

La citotoxicidad de los polifenoles en las células PBMC se determinó con PI por citometría de flujo.

Las células PBMC se plantaron en placas de 96 pocillos y se trataron con diferentes concentraciones de QCT, RES y QCT+RES. Después, las células se estimularon con $50 \mathrm{ng} / \mathrm{mL}$ de anti-CD3 y $10 \mu \mathrm{g} / \mathrm{mL}$ de anti-CD28 y se incubaron durante $96 \mathrm{~h}$ a $37^{\circ} \mathrm{C}$. Las células control se estimularon pero no se trataron con los polifenoles. Después del tiempo de incubación, se añadieron $5 \mu \mathrm{g} / \mathrm{mL}$ de $\mathrm{PI}$ y se analizaron las células con un citómetro FACSCalibur ${ }^{\mathrm{TM}}$ (BD).

\subsubsection{Ensayo de proliferación y viabilidad}

El efecto de los polifenoles sobre la viabilidad y proliferación de las células PBMC se determinó con el compuesto CFSE.

Las células se incubaron con $5 \mu \mathrm{M}$ de CFSE en medio de cultivo sin suero durante $10 \mathrm{~min}$ a $37{ }^{\circ} \mathrm{C}$ en un baño de agua. Después, se añadió $1 \mathrm{~mL}$ de medio de cultivo con $10 \%$ de FCS y se incubaron durante 30 min a temperatura ambiente. Después se recogieron, se plantaron en placas de 96 pocillos, se trataron con los polifenoles y se estimularon con anti-CD3 y anti-CD28 durante $120 \mathrm{~h}$. Finalmente se añadió PI y se analizaron las muestras por citometría de flujo. 


\subsection{Modelo murino de ojo seco}

El efecto anti-inflamatorio de QCT, RES y su combinación (QCT+RES) fue estudiado en un modelo murino de ojo seco seguido de un modelo de transferencia adoptiva. Se evaluaron la tinción corneal con fluoresceína (CFS), la producción lagrimal (TP), el recuento de células caliciformes, los niveles de citoquinas en lágrima y la infiltración de linfocitos $\mathrm{CD} 4^{+} \mathrm{T}$ en conjuntiva.

\subsubsection{Animales}

Se utilizaron dos tipos de ratón: C57BL/6 y C57BL/6 atímicos (sin linfocitos T) de entre 8 y 10 semanas de edad. El estudio con animales fue aprobado por el comité ético de Allergan y se siguieron las normas de "Association for Research in Vision and Ophthalmology (ARVO)".

\subsubsection{Inducción del estrés por desecación y aplicación tópica de los tratamientos.}

El estrés por desecación (DS) se provocó en los ratones C57BL/6 (denominados donantes). Los ratones se colocaron en cajas con rejillas en los laterales y se expusieron a un flujo de aire continuo durante $10 \mathrm{~h}$ al día en una cámara de ambiente controlado, con una humedad relativa del $20 \%$ y una temperatura mantenida de $23{ }^{\circ} \mathrm{C}$ durante 10 días. Además, se administraron $0.1 \mathrm{mg} / \mathrm{día}$ de escopolamina sódica mediante una bomba osmótica implantada subcutáneamente. Los ratones control se mantuvieron en una sala aislada con una humedad relativa del $80 \%$ y una temperatura de $21-23^{\circ} \mathrm{C}$, sin flujo de aire.

Los ratones se dividieron en 6 grupos ( 9 ratones por grupo) de acuerdo al tratamiento seguido:

1) Grupo control (sin DS y sin tratamiento tópico).

2) DS (sin tratamiento tópico).

3) DS + vehículo.

4) $\mathrm{DS}+0.01 \% \mathrm{QCT}$.

5) $\mathrm{DS}+0.1 \%$ RES.

6) $\mathrm{DS}+0.01 \% \mathrm{QCT}+0.1 \%$ RES. 
Los tratamientos comenzaron el día antes de inducir DS y se administraron bilateralmente ( $5 \mu \mathrm{L} /$ ojo) 3 veces al día. Al finalizar el experimento, los ojos izquierdos con los párpados fueron embebidos en OCT y congelados; y los ojos derechos con los párpados sumergidos en paraformaldehído y conservados a $4{ }^{\circ} \mathrm{C}$.

\subsubsection{Tinción corneal}

CFS se evaluó en los ratones donantes después de 9 días de DS. Se instilaron $5 \mu \mathrm{L}$ de $0,125 \%$ de fluoresceína sódica (Sigma) en el saco inferior conjunctival y después de 1 min se examinaron ambas corneas con un microscopio bajo una luz azul cobalto. El punteado superficial se evaluó en las 5 áreas corneales (central, superior, inferior y laterales) según la escala del National Eye Institute.

\subsubsection{Modelo de transferencia adoptiva}

Se aislaron las células $\mathrm{CD}^{+}$de los nódulos linfáticos cervicales y del bazo de los ratones donantes mediante un kit de aislamiento de células CD4 $4^{+}$(MACS System, Miltenyi Biotec, Auburn, CA, EE.UU.). Después se transfirieron a los ratones atímicos (denominados receptores) intraperitonealmente (8 ratones receptores por cada grupo de ratones donantes) y se mantuvieron durante 3 días. Al finalizar el experimento, los ojos izquierdos con los párpados fueron embebidos en OCT y congelados; y los ojos derechos con los párpados sumergidos en paraformaldehído y conservados a $4{ }^{\circ} \mathrm{C}$.

\subsubsection{Producción lagrimal}

TP se midió en los ratones donantes y receptores el día antes de comenzar los experimentos y un día antes de terminarlos. TP se midió con tiras impregnadas de rojo fenol (Zone-Quick; Lacrimedics, Eastsound, WA, EE.UU.). La tira se mantuvo con unas pinzas sobre el canto lateral externo del ojo derecho durante $30 \mathrm{~s}$ y se midió la zona humectada.

\subsubsection{Recogida de lágrima}

La recogida de lágrima se realizó en los ratones donantes los días 6 y 10 y en los receptores el día 3. Se instilaron 1,5 $\mu \mathrm{L}$ de tampón para el ensayo de citoquinas (Millipore) en cada ojo e inmediatamente se recogieron $2 \mu \mathrm{L}(1 \mu \mathrm{L} / \mathrm{ojo})$ de lágrima con 
tampón del menisco lagrimal inferior con un capilar. Las muestras se diluyeron en $8 \mu \mathrm{L}$ de tampón y se almacenaron a $-80{ }^{\circ} \mathrm{C}$.

\subsubsection{Medida de los niveles de citoquinas y quimioquinas en lágrima.}

Se midieron los niveles de 15 citoquinas y quimioquinas (IFN- $\gamma$, IL-1 $\alpha$, IL-1 $\beta$, IL2, IL-4, IL-6, IL-10, IL-12(p70), MMP-9, IL-13, IL-17, IP-10/CXCL10, MCP-1/CCL2, RANTES/CCL5 y TNF- $\alpha$ ) utilizando un ensayo multianalito con tecnología $x-M A P^{\circledR}$ siguiendo el mismo protocolo que el apartado 2.1.7 de esta sección. Las muestras se analizaron con un Luminex 200 (Luminex Corporation).

\subsubsection{Recuento de células caliciformes}

Los ojos derechos con sus respectivos párpados mantenidos en paraformaldehído se fijaron en parafina y se realizaron secciones que posteriormente se tiñeron con ácido de Schiff (PAS). Las secciones se analizaron con un microscopio utilizando un objetivo 20x. El recuento de células caliciformes se realizó en toda la conjuntiva, superior e inferior.

\subsubsection{Inmunohistoquímica}

Los ojos izquierdos con sus respectivos párpados embebidos en OCT se seccionaron con un microtomo en un criostato (CM3050 S; Leica Microsystems, Buffalo Grove, IL, USA). Posteriormente, las secciones se secaron a $37^{\circ} \mathrm{C}$ y se fijaron con acetona a $-20{ }^{\circ} \mathrm{C}$. Después, se bloquearon las peroxidasas endógenas con $\mathrm{H}_{2} \mathrm{O}_{2}$ y se secaron al aire durante $1 \mathrm{~h}$. Se bloquearon las uniones no específicas con suero de conejo y se añadió anticuerpo para CD4. Después de lavar las muestras con PBS se añadió el anticuerpo secundario anti-lgG y, posteriormente, se añadió el reactivo Vectastain Elite ABC (Vector laboratories, Burlingame, CA, EE.UU.). Finalmente, las muestras se incubaron con el sustrato NovaRED (Vector Laboratories) y se realizó una contratinción con hematoxilina (Invitrogen). El recuento de células $\mathrm{CD} 4^{+}$se realizó en toda la conjuntiva, superior e inferior. 


\subsection{Modelo in-vitro de respuesta de mastocitos conjuntivales}

El efecto antialérgico de los compuestos QCT, RES y su combinación fue estudiado en mastocitos derivados de sangre de cordón umbilical humano (CBMC). Se evaluaron la viabilidad celular y la secreción de histamina y citoquinas.

\subsubsection{Reactivos}

El medio Stemspan ${ }^{\mathrm{TM}}$ se adquirió de StemCell Technologies (Grenoble, France). El factor de crecimiento celular (SCF) y las citoquinas IL-3 e IL-6 se adquirieron en Peprotech. FCS se adquirió en Labtech. IgE humano fue comprador en Abcam (Cambridge, MA, USA). PI y anti-IgE humano fueron adquiridos en Sigma.

\subsubsection{Preparación de las disoluciones de los polifenoles}

Las disoluciones de QCT, RES and QCT+RES se prepararon de la misma manera que se describe en los apartados 2.1.2 y 2.2.2 de esta sección.

\subsubsection{Mastocitos derivados de sangre de cordón umbilical}

Las células madre CD34 ${ }^{+}$disponibles comercialmente (Lonza, Wokingham, UK) se cultivaron y diferenciaron como previamente se describió en la bibliografía. ${ }^{293}$

\subsubsection{Sensibilización y activación de los mastocitos}

Las células CBMC se activaron por la unión cruzada de IgE de superficie con anti-lgE siguiendo el protocolo descrito en la bibliografía. ${ }^{292,293}$

\subsubsection{Tratamiento con los polifenoles}

Después de sensibilizar las células CBMC con lgE durante 18 h se añadieron los tratamientos con los polifenoles. Las células se incubaron durante 30 min antes de añadir anti-lgE. Se recogieron sobrenadantes $1 \mathrm{~h}$ y $24 \mathrm{~h}$ después de añadir anti-lgE.

\subsubsection{Ensayo de citotoxicidad}


La citotoxicidad de los polifenoles se determinó $24 \mathrm{~h}$ después de activar las células con anti-lgE. Para ello, se añadieron $5 \mu \mathrm{g} / \mathrm{mL}$ de PI a cada muestra y, seguidamente, las células se analizaron por citometría de flujo (FACSCalibur).

\subsubsection{Secreción de histamina}

La concentración de histamina en los sobrenadantes $1 \mathrm{~h}$ después de la estimulación con anti-lgE se realizó con un kit comercial de ELISA (IBL, Hamburg, Germany) siguiendo las instrucciones del fabricante. En primer lugar, se realizó la acilación de los sobrenadantes y las muestras estándares. Después, se añadió suero de anti-histamina y se reveló con una solución de tetrametilbencidina para, finalmente, medir la absorbancia con un espectrofotómetro.

\subsubsection{Secreción de citoquinas}

Se determinó el nivel de 9 citoquinas (IL-1ß, IL-4, IL-5, IL-8/CXCL8, IL-9, IL-10, IL-13, TNF- $\alpha$ y IFN- $\gamma$ ) en los sobrenadantes a las $24 \mathrm{~h}$ siguiendo el protocolo descrito en el apartado 2.1.7 de esta sección. 


\section{Resultados más relevantes}

1. Quercitina, resveratrol y su combinación tienen un efecto anti-inflamatorio sobre las células del epitelio de la superficie ocular mediante la inhibición de la secreción de citoquinas y quimioquinas, y la expresión de COX-2 en un modelo in-vitro de inflamación ocular.

2. Quercitina, resveratrol y su combinación tienen un efecto antioxidante sobre las células del epitelio de la superficie ocular disminuyendo la formación de especies reactivas de oxígeno cuando las células se expusieron a luz UV-B.

3. La combinación de quercitina y resveratrol tienen un efecto inmunorregulador sobre los linfocitos $T$ inhibiendo la activación y proliferación in-vitro de células mononucleares procedentes de sangre periférica humana.

4. Quercitina, resveratrol y su combinación tienen un efecto anti-alérgico sobre los mastocitos inhibiendo la secreción de histamina y citoquinas in-vitro.

5. La aplicación tópica de quercitina, resveratrol y su combinación disminuye los signos y los niveles de citoquinas en la lágrima de ratones expuestos a estrés por desecación.

6. La aplicación tópica de quercitina y resveratrol evita la infiltración de linfocitos $\mathrm{CD}^{+}$en la conjuntiva de ratones receptores en un modelo de transferencia adoptiva. 


\section{Conclusiones}

1. Quercetina y resveratrol son compuestos naturales con propiedades anti-inflamatorias y antioxidantes en células epiteliales procedentes de la córnea y la conjuntiva humana, y con efectos inmunorreguladores sobre la activación de células del sistema inmune como los linfocitos y los mastocitos.

2. Ambos compuestos han mostrado estas propiedades tanto de forma aislada como en combinación, siendo necesario en el futuro estudiar qué concentraciones de ambos compuestos de forma aislada, o conjuntamente, son más efectivas para su uso.

3. Estas propiedades han demostrado que ambos compuestos pueden ser usados en el tratamiento tópico de enfermedades inflamatorias de la superficie ocular, tales como el ojo seco y la alergia ocular, como han confirmado los resultados obtenidos en el modelo animal de ojo seco. 

XII. Appendix I 

Patente: Composición para su uso en el tratamiento y/o prevención de la inflamación, el estrés oxidativo y la neovascularización ocular. Número de publicación: P201230664. Asignado a: Universidad de Valladolid y Allergan, Inc. Inventores: María Jesús González García, Amalia Enríquez de Salamanca y Aladro, Margarita Calonge Cano, Michael E. Stern y Antonio Abengózar Vela.

COMPOSICIÓN PARA SU USO EN EL TRATAMIENTO Y/O PREVENCIÓN DE LA INFLAMACIÓN, EL ESTRÉS OXIDATIVO Y LA NEOVASCULARIZACIÓN OCULAR

\section{DESCRIPCIÓN}

La presente invención se encuadra en el campo de la oftalmología, específicamente dentro de las composiciones oftálmicas útiles para el tratamiento y/o prevención de lesiones o enfermedades que cursan con inflamación, estrés oxidativo y/o neovascularización ocular, preferiblemente del sistema lagrimal (secretor y excretor), anejos oculares (párpados, incluyendo las glándulas de Meibomio) y de

10 segmento anterior del ojo, el cual comprende conjuntiva (incluyendo las células productoras de mucinas y las glándulas del sistema lagrimal secundario), córnea, limbo esclero-corneal, iris, pupila, cristalino, zónula de Zinn, cuerpo ciliar cámara anterior, humor acuoso, cámara posterior; y más preferiblemente de la superficie ocular, que comprende los epitelios de la córnea, limbo esclero-corneal y conjuntiva, la película lagrimal suprayacente y el estroma subyacente a estas estructuras.

\section{ESTADO DE LA TÉCNICA}

La inflamación ocular es uno de los problemas de mayor incidencia en la patología oftalmológica. E tratamiento de esta afección se puede realizar de forma específica, atacando la etiología, de forma 20 inespecífica, reduciendo los síntomas inflamatorios, o uniendo ambas cosas. En este sentido, los corticosteroides se han utilizado como fármacos de elección en el tratamiento inespecífico de la inflamación ocular pero los efectos adversos que presentan han planteado la necesidad de incrementar el inflamacion ocular, pero los efectos adversos que presentan han planteado la necesidad de incrementar el Pediatr Drugs, 6: 289-301). La actividad antiinflamatoria de estos últimos agentes radica en la inhibición 25 reversible de la actividad de la enzima ciclooxigenasa (COX) en sus isoformas COX-1 y COX-2, impidiendo la formación de prostaglandinas, sustancias mediadoras de la inflamación.

La inflamación ocular puede deberse a diversas causas y tener unas características $u$ otras dependiendo de la zona del ojo en la que se desencadene. Puede estar provocada, por ejemplo, por una intervención quirúrgica sin embargo ésta es solo una de las causas de la patología la cual debe tratarse con quirúrgica, sin embargo, ésta es solo una de las causas de la patología, la cual debe tratarse con
antiinflamatorios en cualquiera de sus manifestaciones para paliar los síntomas y evitar las graves antiinflamatorios en cualquiera de sus manifestaciones para paliar los sintomas y evitar las graves complicaciones dependen de la localización de la inflamación y pueden ir desde la pérdida de las pestañas, si se da en los párpados, empeoramiento del pronóstico de los traumatismos oculares e incluso ceguera en el caso de uveítis o patologia inflamatoria intraocular.

La inflamación puede darse en todas las estructuras de la anatomía ocular. Así, serían susceptibles de verse afectados tanto los anejos oculares como el globo ocular o el nervio óptico, pudiéndose encontrar inflamación tanto de origen infeccioso como no infeccioso $Y$ así, se hablará de inflamaciones orbitarias,

40 palpebrales, intraoculares (uveítis, también denominadas, según las partes a las que afecte predominantemente, retinitis, coroiditis, papilitis o vasculitis) 0 inflamaciones de la superficie ocula (blefaritis, conjuntivitis, queratitis, afecciones del limbo esclero-corneal y patología lagrimal, el síndrome de ojo seco (SOS) incluido)

45 Se estima que alrededor del $7 \%$ de las personas jóvenes padece algún fenómeno de inflamación ocular en España mientras que en la edad adulta la aparición de otras enfermedades que provocan este fenómeno, como el SOS, hace que su prevalencia aumente hasta el $30 \%$ a partir de los 60 años.

El SOS es una enfermedad inflamatoria inmune, crónica, de origen multifactorial que podría originarse en cualquiera de los componentes de la unidad funcional lagrimal, formada por la superficie ocular, las glándulas lagrimales principal y accesorias y la inervación de interconexión entre ellas -ramas aferentes y eferentes-, y que, en algún momento, provoca daño en la superficie ocular (epitelios de córnea, limbo esclero-corneal y conjuntiva, película lagrimal suprayacente y estroma subyacente a estas estructuras) que se detectan con las pruebas convenientes.

En cuanto a la neovascularización ocular, ésta se encuentra ligada directamente al estímulo de la hipoxia a la necrosis tisular y a la activación de complejas cascadas de interacción entre citoquinas/quimioquinas (Ej: IL-8) y factores de crecimiento estimuladores de la formación de neovasos (VEGF, bFGF, TGF-o, PDGF, IGF). Todos estos eventos son desencadenados, más frecuentemente, por inflamación intensa y/o

60 prolongada y/o recurrente, independientemente de la causa que la origine. Por ejemplo, un evento agudo y muy intenso como una causticación (quemadura química) o un evento menos intenso pero recurrente (queratitis herpética) o prolongado (SOS severo) podrán ser el origen de una neovascularización corneal. (queratitis herpética) o prolongado (SOS severo) podrán ser el origen de una neovascularización corneal. 
directa entre citoquinas, factores de crecimiento, moléculas de adhesión celular (integrinas) y metaloproteinasas (MMP). Dichas MMP cumplen un rol esencial en la invasión tisular por neovasos en diferentes enfermedades corneales (inflamatorias, autoinmunes, infecciosas), por lo que la inhibición de las MMP por los inhibidores naturales (o TIMPs) y por drogas inhibidoras de MMP, resultaría en la 5 inhibición de la neovascularización.

Uno de los casos en que puede aparecer neovascularización es, por ejemplo, cuando se produce la destrucción extensa de la superficie corneal, y en especial de las células madre pluripotenciales localizadas a nivel del limbo esclero-corneal, lo que llevaría a la pérdida de la re-epitelización corneal

10 normal y finalmente a una conjuntivalización o pannus corneal con una invasión por neovasos. Este proceso, en la actualidad, se define como Síndrome de Insuficiencia Límbica (SIL).

Los hallazgos experimentales (in vitro e in vivo), que permiten una mejor comprensión de la biología molecular de la neovascularización y cicatrización, han ayudado a realizar nuevos tratamientos médicos 5 con drogas que inhiben factores de crecimiento angiogénicos o MMP, así como con el uso de nuevos factores de crecimiento que inhiben la neovascularización ("Pigment Epithelium Derived Factor" o PEDF), y mediante tratamientos quirúrgicos tales como el trasplante de células limbares y membrana amniótica.

En lo que se refiere al estrés oxidativo, las especies de oxígeno reactivas (ROS), que producen efectos 20 adversos a nivel celular, se generan por múltiples mecanismos endógenos, como por ejemplo, las reacciones inflamatorias o la isquemia; y exógenos, como por ejemplo, la exposición a la luz ultravioleta. A nivel ocular, cabe destacar por ejemplo el proceso inflamatorio de la unidad funcional lagrimal que se A nivel ocular, cabe destacar por ejemplo el proceso inflamatorio de la unidad funcional lagrimal que
produce en el SOS, el cual tiene asociado un proceso oxidativo que tiene lugar en la superficie ocular.

25 En referencia a la película lagrimal, la capa lipídica está formada por ésteres de colesterol y fosfolípidos Si tenemos en cuenta que la capa lipídica está en contacto directo con el aire y los procesos de oxidación que sufren los lípidos en presencia de moléculas radicalarias, la generación de ROS en el proceso oxidativo inflamatorio puede degradar los ácidos grasos, aumentado la evaporación de la capa acuosa de la película lagrimal. Esto se corrobora con el aumento de la concentración de peroxidasa lipídica en la 30 superficie ocular.

Además de las ROS, existe también la formación de especies oxidantes relativas al nitrógeno. El óxido nítrico y las especies oxidantes relativas al nitrógeno pueden tener un papel muy importante en el SOS La expresión de la óxido nítrico sintasa 2 y 3 (NOS2, NOS3) en el epitelio conjuntival está aumentada en 35 el SOS respecto al epitelio conjuntival de ojos sanos, lo que agrava la sintomatología.

Este proceso oxidativo produce una elevada concentración, y por ello una elevada actividad, de mieloperoxidasa (MPO) y xantina oxidoreductasa/xantina oxidasa en el epitelio conjuntival entre otras. Dichas enzimas generan especies radicalarias que podrían estar implicadas en los procesos oxidativos 40 que ocurren en el SOS. Además, la concentración de las enzimas antioxidantes, encargadas de neutralizar las moléculas radicalarias, se encuentra disminuida en el ojo seco, lo que podría contribuir aun más al daño oxidativo producido en el proceso inflamatorio.

Por otro lado, los compuestos polifenólicos, dentro de los cuales se encuentran los polifenoles flavonoides 45 y no flavonoides, son compuestos cuya característica principal es la presencia de grupos bencilo hidroxilo en su estructura y cuyas propiedades antioxidantes han sido ampliamente estudiadas. Dentro de hidroxilo en su estructura y cuyas propiedades antioxidantes han sido ampliamente estudiadas. Dentro de
los compuestos polifenólicos flavonoides destaca la molécula quercetina (QCT), 3,3',4',5,7-pentahidroxi-2 los compuestos polifenólicos flavonoides destaca la molécula quercetina (QCT), 3,3',4',5,7-pentahidroxi-2-
fenilcromen-4-ona. Es el compuesto flavonoide más abundante, con muchos efectos potencialmente beneficiosos para la salud humana (Kelly GS, 2011. Alternative Medicine Review, 16(2):172-194). De

50 mismo modo, dentro de los compuestos polifenólicos no flavonoides, dentro de los cuales se encuentran los estilbenoides que son compuestos cuya característica principal es la presencia de grupos bencilo hidroxilo en su estructura cabe destacar una molécula cuya importancia ha aumentado en los últimos años debido

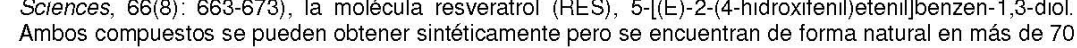
especies de plantas, frutas y verduras.

En cuanto a su aplicación clínica, tanto los polifenoles en general como los derivados del estilbeno o estilbenoides en particular son moléculas utilizadas para diferentes tratamientos. En este sentido, algunos 60 estudios indican que RES presenta varios beneficios para la salud, tales como la prevención de problemas cardiovasculares, oncológicos e inflamatorios (Das S., Das D. K., 2007, inflamm Allergy Drug Targets, $6(3): 168-173$ ). Por estos motivos, se han descrito algunas composiciones oftálmicas que 
comprenden compuestos polifenólicos para el tratamiento de la inflamación ocular, como por ejemplo, la que se describe en US20060127505

Sin embargo, debido a que un gran número de patologías y lesiones oculares cursan frecuentemente con 5 procesos inflamatorios, estrés oxidativo y/o neovascularización ocular, continúa existiendo como objetivo en el campo de la oftalmología la identificación de compuestos y composiciones oftálmicas que permitan tratar y/o prevenir eficientemente dichos procesos patológicos.

\section{DESCRIPCIÓN DE LA INVENCIÓN}

10

La presente invención propone el uso de la quercetina ( $Q C T)$, o de cualquiera de sus sales o derivados, para el tratamiento y/o prevención de la inflamación, del estrés oxidativo y/o de la neovascularización ocular, preferiblemente del segmento anterior, más preferiblemente de la superficie ocular, y aun más preferiblemente del epitelio corneal. Además, la presente invención proporciona una composición, de ahora en adelante "composición de la invención", que comprende QCT, o cualquiera de sus sales o derivados, y al menos otro compuesto polifenólico, o cualquiera de sus sales o derivados, preferiblemente resveratrol (RES), la cual también es de utilidad en dicha aplicación clínica.

La QCT, o cualquiera de sus sales o derivados, así como la composición de la invención, son por tanto ocular, preferiblemente del segmento anterior, más preferiblemente de la superficie ocular, y aun más preferiblemente del epitelio corneal. Como demuestran los ejemplos de la presente invención, tanto la QCT sola como dicha composición son capaces de reducir los niveles de, por ejemplo, aunque sin limitarnos, citoquinas, quimioquinas, ciclooxigenasa 2 (COX-2) y especies oxidativas en, por ejemplo,

25 aunque sin limitarnos, células de epitelio corneal humano (HCE) estimuladas con TNF- $\alpha$ o irradiadas con luz ultravioleta. También son capaces de disminuir significativamente la cantidad de linfocitos CD4+ en luz ultravioleta. También son capaces de disminuir significativam
conjuntiva en modelos animales de síndrome de ojo seco (SOS)

Por ello, un primer aspecto de la invención se refiere al uso de la QCT, o de cualquiera de sus sales o derivados, para la elaboración de un medicamento para el tratamiento y/o prevención de la inflamación ocular, preferiblemente de la superficie ocular, más preferiblemente del epitelio corneal. Alternativamente, este aspecto de la invención se refiere a la QCT, o a cualquiera de sus sales o derivados, para su uso como medicamento en el tratamiento y/o prevención de la inflamación ocular, preferiblemente de la superficie ocular, más preferiblemente del epitelio corneal.

La "quercetina" o "QCT" o "3,3',4',5,7-pentahidroxi-2-fenilcromen-4-ona" es un compuesto polifenólico flavonoide y natural, que se encuentra en altas concentraciones en una gran variedad de especies vegetales $y$, por tanto, en alimentos tales como, por ejemplo aunque sin limitarnos, fruta, verduras cereales, té o vino tinto; aunque también puede ser sintetizada químicamente. Su fórmula molecular es

$40 \quad \mathrm{C}_{15} \mathrm{H}_{10} \mathrm{O}_{7}$ y su estructura química (I) es la que se indica a continuación:<smiles>O=c1c(O)c(-c2ccc(O)c(O)c2)oc2cc(O)cc(O)c12</smiles>

Así, el medicamento que comprende QCT, o cualquiera de sus sales o derivados, es de utilidad para el tratamiento y/o prevención de enfermedades o lesiones que cursan con inflamación ocular.

Otro aspecto de la invención se refiere al uso de la QCT, o de cualquiera de sus sales o derivados, para la elaboración de un medicamento para el tratamiento y/o prevención del estrés oxidativo ocular, 50 preferiblemente de la superficie ocular, más preferiblemente del epitelio corneal. Alternativamente, este 
aspecto de la invención se refiere a la QCT, o a cualquiera de sus sales o derivados, para su uso como medicamento en el tratamiento y/o prevención del estrés oxidativo ocular, preferiblemente de la superficie ocular, más preferiblemente del epitelio corneal.

5 Así, el medicamento que comprende QCT, o cualquiera de sus sales o derivados, es de utilidad para el tratamiento y/o prevención de enfermedades o lesiones que cursan con estrés oxidativo ocular.

Otro aspecto de la invención se refiere al uso de la QCT, o de cualquiera de sus sales o derivados, para la elaboración de un medicamento para el tratamiento y/o prevención de la neovascularización ocula

10 preferiblemente del segmento anterior, más preferiblemente de la superficie ocular, aun más preferiblemente de la córnea. Alternativamente, este aspecto de la invención se refiere a la QCT, 0 cualquiera de sus sales o derivados, para su uso como medicamento en el tratamiento yio prevención de la neovascularización ocular preferiblemente del segmento anterior, más preferiblemente de la superficie ocular, aun más preferiblemente de la córnea.

15

Así, el medicamento que comprende QCT, o cualquiera de sus sales o derivados, es de utilidad para el tratamiento y/o prevención de enfermedades o lesiones que cursan con neovascularización ocular.

En otra realización preferida, el medicamento que comprende QCT, o cualquiera de sus sales o 20 derivados, es para el tratamiento y/o prevención de síndrome de ojo seco (SOS), blefaritis, disfunción de las glándulas de Meibomio, meibomitis, procesos alérgicos oculares, distrofias corneales, conjuntivitis, las glándulas de Meibomio, meibomitis, procesos alérgicos oculares, distrofias corneales, conjuntivitis, alteración de la superficie ocular por el uso de lentes de contacto, enfermedades autoinmunes que
afecten al segmento anterior del ojo, postcirugía del segmento anterior, quemaduras (tanto químicas o "causticaciones" como térmicas) o lesiones o patologías oculares producidas por radiación ultravioleta.

25 Más preferiblemente, el medicamento que comprende QCT, o cualquiera de sus sales o derivados, es para el tratamiento y/o prevención de SOS.

Otro aspecto de la invención se refiere al uso de la QCT, o de cualquiera de sus sales o derivados, como agente antimicrobiano antioxidante y antiinflamatorio en soluciones de limpieza y mantenimiento de

30 lentes de contacto. Otro aspecto de la invención se refiere a una solución para la limpieza mantenimiento de lentes de contacto que comprende QCT o cualquiera de sus sales o derivados.

Un segundo aspecto de la invención se refiere a una composición, "composición de la invención", que comprende QCT, o cualquiera de sus sales o derivados, y al menos otro compuesto polifenólico, cualquiera de sus sales o derivados.

El término "otro compuesto polifenólico" empleado en la presente invención hace referencia a un metabolito secundario biosintetizado por, aunque sin limitarnos, organismos del reino vegetal que se puede encontrar en, por ejemplo, alimentos derivados de fuentes vegetales, pero que también se puede 40 obtener sintéticamente, y que posee más de un grupo fenol. Los polifenoles son un grupo de moléculas cuya característica principal es la presencia de grupos bencilo e hidroxilo en su estructura. Estos polifenoles se pueden clasificar de forma general como: ácidos fenólicos, flavonoides, estilbenos y lignanos. A su vez, los compuestos flavonoides se pueden subclasificar como chalconas, flavonoles, flavonas, flavanonas, antocianidinas e isoflavonoides. El otro compuesto polifenólico de la composición de

45 la invención puede ser, aunque sin limitarnos, cualquier compuesto polifenólico dentro de las cuatro familias. Por ello, en una realización preferida, el otro compuesto polifenólico comprendido en la familias. Por ello, en una realización preferida, el otro compuesto polifenólico comprendido en la
composición de la invención es un estilbenoide, más preferiblemente el RES, o cualquiera de sus sales 0 derivados

50 Un "compuesto estilbenoide" es cualquier compuesto cuya estructura principal sea la molécula de estilbeno (Il), tales como por ejemplo, aunque sin limitarnos, resveratrol, piceid piceatanol, oxiresveratrol, rhapontigenin o pterostilbeno. 


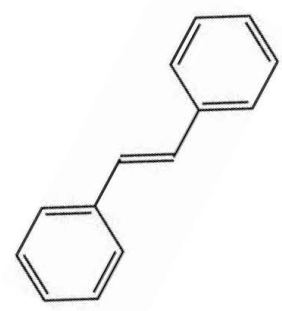

(II)

5 El "resveratrol", "RES" ó "5-[(E)-2-(4-hidroxifenil)etenil]benzen-1,3-diol" se encuentra en una gran variedad de plantas, aunque también puede ser sintetizado químicamente. Su número CAS es 501-36-0, su fórmula molecular es $\mathrm{C}_{14} \mathrm{H}_{12} \mathrm{O}_{3}$ y su estructura química (III) es la que se indica a continuación:<smiles>Oc1ccc(/C=C/c2cc(O)cc(O)c2)cc1</smiles>

(III)

10

Tal como aquí se utiliza, el término "derivado" incluye a compuestos farmacéuticamente aceptables, es decir, derivados de la QCT o del otro compuesto polifenólico comprendido en la composición de la invención, preferiblemente RES, que pueden ser utilizados en la elaboración de la composición de la invención, y a derivados farmacéuticamente no aceptables, ya que éstos pueden ser útiles en la 15 preparación de derivados farmacéuticamente aceptables.

En una realización más preferida, la composición de la invención además comprende un vehículo farmacéuticamente aceptable. Además, dicha composición puede comprender uno o más excipientes.

20 El término "excipiente" hace referencia a una sustancia que ayuda a la absorción de los elementos de la composición de la invención, estabiliza dichos elementos, activa o ayuda a la preparación de la composición en el sentido de darle consistencia o aportar sabores que la hagan más agradable. Así pues, los excipientes podrían tener la función de mantener los ingredientes unidos, como por ejemplo es el caso de almidones, azúcares o celulosas, la función de endulzar, la función de colorante, la función de de almidones, azúcares o celulosas, la función de endulzar, la función de colorante, la función de
protección de la composición, como por ejemplo, para aislarla del aire y/o la humedad, la función de relleno de una pastilla, cápsula o cualquier otra forma de presentación, la función desintegradora para facilitar la disolución de los componentes y su absorción en el intestino, sin excluir otro tipo de excipientes no mencionados en este parrafo.

30 El "vehículo farmacéuticamente aceptable", al igual que el excipiente, es una sustancia que se emplea en la composición para diluir cualquiera de los componentes comprendidos en ella hasta un volumen o peso leterminado. El vehículo farmacéuticamente aceptable es una sustancia inerte o de acción análoga a determinado. El vehículo farmacéuticamente aceptable es una sustancia inerte o de acción análoga a vehículo es facilitar la incorporación de otros elementos, permitir una mejor dosificación y administración o dar consistencia y forma a la composición. 
Preferiblemente, la composición de la invención comprende QCT, o cualquiera de sus sales o derivados, y al menos otro compuesto polifenólico, o cualquiera de sus sales o derivados, preferiblemente RES, en una cantidad terapéuticamente efectiva, entendiéndose por "cantidad terapéuticamente efectiva" el nivel cantidad o concentración de OCT, 0 de cualquiera de sus saes o derivados, y de al menos otro 5 compuesto polifeńlico, o de cualquiera dr compuesto polfenćlico, o de cualquiera de sus sales o derivados, prefenblemente de RES, que produzca el efecto deseado tratando y/o previniendo la inflamación, el estrés oxidativo y/o la neovascularización ocular, sin causar efectos adversos. La dosificación para obtener una cantidad terapéuticamente efectiva depende de una variedad de factores, como por ejemplo, la edad, peso, sexo o tolerancia del individuo al

a composición de la presente invención puede formularse para su administración en una variedad de formas conocidas en el estado de la técnica. Como ejemplos de preparaciones se incluye cualquier composición sólida (comprimidos, píldoras, cápsulas, gránulos, etc.) o líquida (soluciones, suspensiones o emulsiones) para administración oral, tópica o parenteral. La composición de la presente invención emulsiones) para administración oral, tópica o parenteral. La composición de la presente invención
también puede estar en forma de formulaciones de liberación sostenida de drogas o de cualquier otro también puede estar en forma de formulaciones de liberación sostenida de drogas o de cualquier otro
sistema convencional de liberación, así puede estar contenida, aunque sin limitarnos, en nanopartículas, liposomas o nanosferas, en un material polimérico, en un implante biodegradable o no biodegradable o en micropartículas biodegradables, como por ejemplo, microesferas biodegradables.

20 Tal composición y/o sus formulaciones pueden administrarse a un animal, incluyendo un mamífero y, por tanto, al hombre en una variedad de formas, incluyendo, pero sin limitarse, intraperitoneal, intravenosa intradérmica, intraespinal, intraestromal, intraarticular, intrasinovial, intratecal, intralesional, intraarterial, intradérmica, intraespinal, intraestromal, intraarticular, intrasinovial, intratecal, intralesional, intraarterial, intramuscular, intranasal, intracraneal, subcutánea, intraorbital, intracapsular, tópica, mediante parches
transdérmicos, percutánea, espray nasal, implante quirúrgico, pintura quirúrgica interna o bomba de 25 infusión.

En una realización aun más preferida, la composición de la invención se encuentra formulada para su administración oftálmica. La expresión "formulada para su administración oftálmica" se refiere a una formulación que permita que la composición de la invención pueda ser administrada ocularmente, por 30 ejemplo aunque sin limitarnos, de manera tópica o de manera intraocular, sin que dicha administración ejemplo aunque sin limitarnos, de manera tópica o de manera intraocular, sin que dicha administración composición de la invención formulada para su administración oftálmica son, aunque sin limitarnos, dicha composición asociada a agua, a sales, a un vehículo líquido polimérico o semi-sólido, a un tampón fosfato o a cualquier otro vehículo líquido oftálmicamente aceptable de los conocidos en el estado de la técnica.

35

Como se ha explicado anteriormente, la composición de la invención es capaz de reducir los niveles de por ejemplo, aunque sin limitarnos, citoquinas, quimioquinas y COX-2 factores implicados en el proceso inflamatorio en células oculares que presentan un fenotipo inflamado así como de especies oxidativas en inflamatorio, en células oculares que presentan un fenotipo inflamado, así como de especies oxidativas en células oculares que han sido inducidas a sufrir estrés oxidativo. Por ello, la composición de la invención ocular, preferiblemente del segmento anterior, más preferiblemente de la superficie ocular, y aun más preferiblemente del epitelio corneal.

Así, otro aspecto de la invención se refiere al uso de la composición de la invención para la elaboración 5 de un medicamento $o$, alternativamente, a la composición de la invención para su uso como medicamento, de ahora en adelante, "medicamento de la invención".

Los "medicamentos" a los que se refiere la presente invención pueden ser de uso humano o veterinario. El "medicamento de uso humano" es toda sustancia o combinación de sustancias que se presente como

50 poseedora de propiedades para el tratamiento o prevención de enfermedades en seres humanos o que pueda usarse en seres humanos o administrarse a seres humanos con el fin de restaurar, corregir modificar las funciones fisiológicas ejerciendo una acción farmacológica inmunológica o metabólica o de establecer un diagnóstico médico. El "medicamento de uso veterinario" es toda sustancia o combinación de sustancias que se presente como poseedora de propiedades curativas o preventivas con respecto a

55 las enfermedades animales o que pueda administrarse al animal con el fin de restablecer, corregir 0 modificar sus funciones fisiológicas ejerciendo una acción farmacológica, inmunológica o metabólica, o de establecer un diagnóstico veterinario.

En una realización preferida de este aspecto de la invención, el medicamento es para el tratamiento y/o 60 prevención de la inflamación ocular.

Se entiende por "inflamación ocular" la inflamación producida en cualquier estructura ocular, incluidas todas las estructuras del segmento anterior, del segmento posterior del ojo y los anejos oculares, por 
ejemplo, aunque sin limitarnos, en el nervio óptico, párpados, glándula lagrimal principal, conducto hialoideo, retina, coroides, esclera, musculatura ocular o en cualquier estructura que forme parte de segmento anterior, incluyendo la superficie ocular. Se entiende por "segmento anterior" cualquier estructura seleccionada de la lista que comprende: conjuntiva cornea limbo esclerocorneal iris pupila, 5 cristalino zónula de Zinn, cuerpo cliar, camprán an crialino, zónula de Meibomio, glándulas mucosas o aparato lagrimal (glándulas lagrimales, a excepción de la glándula lagrimal principal, conducto nasolagrimal y saco lagrimal). La inflamación ocular cursa con síntomas tales como por ejemplo, aunque sin limitarnos, dolor, enrojecimiento e hinchazón del tejido afectado, así como con la sobreexpresión de factores implicados en el proceso inflamatorio tales como, por ejemplo aunque

10 sin limitarnos, IL-6, IL-8, IP-10, VEGF, TNF- $a$, COX-1 o COX-2

En una realización más preferida, el medicamento de la invención es para el tratamiento y/o prevención de la inflamación del segmento anterior y/o de la glándula lagrimal principal y/o párpados En una realización aun más preferida, el medicamento de la invención es para el tratamiento y/o prevención de la realización aun más preferida, el medicamento de la invención es para el tratamiento y/o prevención de la
inflamación de la superficie ocular. Se entiende por "superficie ocular" cualquier estructura seleccionada inflamación de la superficie ocular. Se entiende por "superficie ocular" cualquier estructura seleccionada
de la lista que comprende: epitelios de la córnea, limbo esclero-corneal o conjuntiva, película lagrimal suprayacente o estroma subyacente a estas estructuras. En una realización aun más preferida, el medicamento de la invención es para el tratamiento y/o prevención de la inflamación del epitelio cornea.

20 La inflamación ocular está asociada a una diversidad de enfermedades y lesiones oculares, tales como por ejemplo aunque sin limitarnos Síndrome de Insuficiencia Límbica (SIL) SOS blefaritis disfunción de las glándulas de Meibomio, meibomitis, procesos alérgicos oculares, conjuntivitis, alteración de las glándulas de Melbomio, meibomitis, procesos alérgicos oculares, conjuntivitis, alteración de la
superficie ocular, preferiblemente del epitelio corneal, provocada por el uso de lentes de contacto y de sus superficie ocular, preferiblemente del epitelio corneal, provocada por el uso de lentes de contacto y de sus
sistemas de limpieza y mantenimiento, enfermedades autoinmunes, preferiblemente que afectan al segmento anterior del ojo, como por ejemplo aunque sin limitarnos, síndrome de Sjögren, postcirugía preferiblemente del segmento anterior del ojo, quemaduras (tanto químicas o causticaciones, como térmicas) o lesiones o patologías oculares producidas por radiación ultravioleta. Por ello, el medicamento de la invención es de utilidad para el tratamiento y/o prevención de enfermedades o lesiones que cursan con inflamación ocular, preferiblemente, de las indicadas en este párrafo.

30

En otra realización preferida, el medicamento de la invención es para el tratamiento y/o prevención del estrés oxidativo ocular

Se entiende por "estrés oxidativo ocular" la condición de citotoxicidad que es consecuencia de un 35 desequilibrio entre la producción de radicales libres y la capacidad de la célula de defenderse contra ellos, por lo que está causada por un incremento en la formación de dichos radicales libres o por una disminución de los agentes que actúan como antioxidantes, o por ambos motivos conjuntamente. $E$ estrés oxidativo ocular puede producirse en cualquier estructura ocular incluidas todas las estructuras del estrés oxidativo ocular puede producirse en cualquier estructura ocular, incluidas todas las estructuras de segmento anterior, del segmento posterior del ojo y los anejos oculares, por ejemplo, aunque sin
limitarnos, en el nervio óptico, glándula lagrimal principal, conducto hialoideo, retina, coroides, esclera, limitarnos, en el nervio óptico, glándula lagrimal principal, conducto hialoideo, retina, coroides, esclera,
musculatura ocular o en cualquier estructura que forme parte del segmento anterior, incluyendo la superficie ocular. En una realización más preferida, el medicamento de la invención es para el tratamiento y/o prevención del estrés oxidativo del segmento anterior. En una realización aun más preferida, el medicamento de la invención es para el tratamiento y/o prevención del estrés oxidativo de la supericie ocular. En una realización aun más preferida, el medicamento de la invención es para el tratamiento y/o prevención del estrés oxidativo del epitelio corneal.

El estrés oxidativo ocular está asociado a una diversidad de lesiones y enfermedades oculares, tales como por ejemplo, aunque sin limitarnos, pterigium, cataratas, distrofias corneales, glaucoma, retinopatía

50 diabética, degeneración macular, SOS, SIL, blefaritis, disfunción de las glándulas de Meibomio, meibomitis, procesos alérgicos oculares, conjuntivitis, alteración de la superficie ocular, preferiblemente del epitelio corneal, provocada por el uso de lentes de contacto y de sus sistemas de limpieza y mantenimiento enfermedades autoinmunes, preferiblemente que afectan al segmento anterior del ojo, como por ejemplo aunque sin limitarnos, sindrome de Sjögren, postcirugía, preferiblemente del segment anterior del ojo, quemaduras (tanto químicas o causticaciones, como térmicas) o lesiones o patologías oculares producidas por radiación ultravioleta o por cualquier otro factor endógeno, como por ejemplo aunque sin limitarnos, reacciones inflamatorias, o exógeno, como por ejemplo aunque sin limitarnos, humo del tabaco o contaminantes ambientales, capaz de inducir formación de especies radicalarias. Por ello, el medicamento de la invención es de utilidad para el tratamiento y/o prevención de enfermedades

60 lesiones que cursan con estrés oxidativo ocular, preferiblemente, de las indicadas en este párrafo.

En otra realización preferida, el medicamento de la invención es para el tratamiento y/o prevención de la neovascularización ocular. 
Se entiende por "neovascularización ocular" la formación de nuevos vasos sanguíneos en las estructuras oculares, por ejemplo aunque sin limitarnos, en la retina, papila óptica, coroides o en cualquier estructura que forme parte del segmento anterior, incluyendo la superficie ocular, preferiblemente en córnea conjuntiva limbo esclerocorneal o iris. En una realización más preferida el medicamento de la invención es para el tratamier es parás preferida, yloprevención de la neovasculaización del segmento anterior En una realización aun más preferida, el medicamento de la invención es para el tratamiento y/o prevención de la neovascularización de la superficie ocular. En una realización aun más preferida, el medicamento de la invención es para el tratamiento y/o prevención de la neovascularización de la córnea.

10 La neovascularización ocular está asociada a una diversidad de lesiones y enfermedades oculares, tales como por ejemplo, aunque sin limitarnos, conjuntivitis, alteración de la superficie ocular, preferiblemente del epitelio corneal, provocada por el uso de lentes de contacto y de sus sistemas de limpieza y mantenimiento enfermedades autoinmunes (como síndrome de Stevens-Johnson, Lyell, penfigoide de las membranas mucosas, etc), preferiblemente que afectan al segmento anterior, postcirugía 5 preferiblemente del segmento anterior, quemaduras (tanto químicas o causticaciones, como térmicas) 0 lesiones o patologías oculares producidas por radiación ultravioleta. Por ello, el medicamento de la invención es de utilidad para el tratamiento y/o prevención de enfermedades o lesiones que cursan con neovascularización ocular, preferiblemente, de las indicadas en este párrafo.

20 En una realización aun más preferida, el medicamento de la invención es para el tratamiento y/o prevención de SOS, blefaritis, disfunción de las glándulas de Meibomio, meibomitis, procesos alérgicos oculares, distrofias corneales, conjuntivitis, alteración de la superficie ocular por el uso de lentes de oculares, distrofias corneales, conjuntivitis, alteración de la superficie ocular por el uso de lentes de
contacto, enfermedades autoinmunes que afectan al segmento anterior del ojo, postcirugía del segmento anterior, quemaduras (tanto químicas o "causticaciones" como térmicas) o lesiones o patologías oculares 5 producidas por radiación ultravioleta. Aun más preferiblemente, el medicamento de la invención es para el tratamiento y/o prevención de SOS (queratoconjuntivitis seca, queratitis seca o xeroftalmia), el cual se ha definido como una enfermedad multifactorial de la superficie ocular, incluyendo la película lagrimal, que causa síntomas de incomodidad, perturbación visual e inestabilidad lagrimal con un daño potencial a superficie ocular, acompañado de un aumento de la osmolaridad de la película agrimal e infamación de la

30 superficie ocular. Dicho síndrome se puede diagnosticar por ejemplo, aunque sin limitarnos, mediante un examen con lámpara de hendidura de la película lagrimal, durante el cual se puede colocar un colorante en el ojo, como la fluoresceína, para hacer que dicha película sea más visible y así poder evaluar su estabilidad, o bien mediante la prueba del test de Schirmer, la cual mide la tasa de producción de lágrimas usando una tira de papel de filtro que se coloca en el extremo del párpado y mide la cantidad de lágrima

35 que produce el ojo.

Por otro lado la limpieza y mantenimiento de las lentes de contacto es fundamental para evitar posibles patologías oculares derivadas de su uso. Los sistemas de limpieza y mantenimiento deben cumplir una patologías oculares derivadas de su uso. Los sistemas de limpieza y mantenimiento deben cumplir una serie de requisitos como son no alterar ni irritar los tejidos oculares, no alterar ni interferir en la fisiología
normal del ojo, no alterar ni dañar las lentes de contacto, evitar la contaminación por microorganismos de las lentes de contacto y mantener éstas lo más limpias posible. Tanto la QCT sola como la composición de la invención cumplen todos estos requisitos, y además presentan las características que debe cumplir un agente desinfectante de este tipo: $\mathrm{pH}$ y tonicidad similares a la lágrima, bacteriostático y/o bactericida, soluble en agua y estable en solución acuosa y en frascos de plástico.

45

Por todo ello, otro aspecto de la invención se refiere al uso de la composición de la invención como agente antimicrobiano, antioxidante y antiinflamatorio en soluciones de limpieza y mantenimiento de lentes de contacto. Otro aspecto de la invención se refiere a una solución para la limpieza y mantenimiento de lentes de contacto que comprende la composición de la invención.

50

Un "agente antimicrobiano" es aquel compuesto químico, o mezcla de compuestos, que inhibe e crecimiento o mata a los microorganismos. El agente antimicrobiano, tal y como se entiende en la presente invención, puede ser aunque sin limitarnos, antibacteriano (dirigido contra bacterias) antifúngico (dirigido contra hongos) o antivírico (dirigido contra virus) y puede ser estático, que inhibe el crecimiento

55 del microorganismo sin llegar a provocar su muerte, por ejemplo, bacteriostático o fungistático, o puede destruir a los microorganismos, por ejemplo, bactericida o fungicida.

Dentro de las "soluciones de limpieza y mantenimiento de lentes de contacto" se incluyen, aunque sin limitarnos, las soluciones limpiadoras, las soluciones conservadoras o humectantes, cuya función es

60 guardar y almacenar las lentes de contacto cuando no están en uso, las soluciones acondicionadoras, los sistemas de peróxidos y los sistemas de solución única, que realizan una limpieza mecánica y desinfectante y a su vez actúan como conservantes y humectantes de las lentes. 
Tal y como se utiliza en la presente invención, el término "lentes de contacto" se refiere tanto a las lentes de contacto duras, incluyendo las rígidas y las gas permeable o semirrígidas, como a las blandas 0 hidrofílicas

5 A lo largo de la descripción y las reivindicaciones la palabra "comprende" y sus variantes no pretenden excluir otras características técnicas, aditivos o componentes. Para los expertos en la materia, otros objetos, ventajas y características de la invención se desprenderán en parte de la descripción y en parte de la práctica de la invención. Los siguientes ejemplos y dibujos se proporcionan a modo de ilustración, y no se pretende que sean limitativos de la presente invención.

10

\section{DESCRIPCIÓN DE LAS FIGURAS}

Fig. 1. Muestra la cantidad de IL-6 producida en células de epitelio corneal humano (HCE) estimuladas con $25 \mathrm{ng} / \mathrm{mL}$ de TNF- $\alpha$ y tratadas con vehículo $(0,5 \%$ de etanol (EtOH)), $50 \mu \mathrm{M}$ de 15 resveratrol (RES), $25 \mu \mathrm{M}$ de quercetina (QCT), $25 \mu \mathrm{M}$ de RES + $25 \mu \mathrm{M}$ de QCT $\circ 50 \mu \mathrm{M}$ de RES + 25 $\mu \mathbf{M}$ de QCT, durante $\mathbf{2 4}$ horas. NOTA: las células fueron tratadas previamente a su estimulación durante 2 horas con el tratamiento correspondiente. Control: células de HCE no estimuladas con TNF- $\alpha$ y tratadas con vehículo. ${ }^{*}: p<0.05$

20 Fig. 2. Muestra la cantidad de IL-8 producida en células de epitelio corneal humano (HCE) estimuladas con $25 \mathrm{ng} / \mathrm{mL}$ de TNF- $\alpha$ y tratadas con vehículo $(0,5 \%$ de etanol $(\mathrm{EtOH})), 50 \mu \mathrm{M}$ de resveratrol (RES), $25 \mu \mathrm{M}$ de quercetina (QCT), $25 \mu \mathrm{M}$ de RES + $25 \mu \mathrm{M}$ de QCT o $50 \mu \mathrm{M}$ de RES + 25 $\mu \mathbf{M}$ de QCT, durante $\mathbf{2 4}$ horas. NOTA: las células fueron tratadas previamente a su estimulación durante 2 horas con el tratamiento correspondiente. Control: células de HCE no estimuladas con TNF- $\alpha$ y tratadas con vehículo. *: $p<0,05$

Fig. 3. Muestra la cantidad de IP-10 producida en células de epitelio corneal humano (HCE) estimuladas con $25 \mathrm{ng} / \mathrm{mL}$ de TNF- $\alpha$ y tratadas con vehículo $(0,5 \%$ de etanol (EtOH)), $50 \mu \mathrm{M}$ de resveratrol (RES), $25 \mu \mathrm{M}$ de quercetina (QCT), $25 \mu \mathrm{M}$ de RES + $25 \mu \mathrm{M}$ de QCT o $50 \mu \mathrm{M}$ de RES + 25

$30 \mu \mathrm{M}$ de QCT, durante $\mathbf{2 4}$ horas. NOTA: las células fueron tratadas previamente a su estimulación durante 2 horas con el tratamiento correspondiente. Control: células de HCE no estimuladas con TNF- $\alpha$ y tratadas con vehículo. *: $p<0,05$

Fig. 4. Muestra la cantidad de VEGF producida en células de epitelio corneal humano (HCE) 35 estimuladas con $25 \mathrm{ng} / \mathrm{mL}$ de TNF- $\alpha$ y tratadas con vehiculo $(0,5 \%$ de etanol (EtOH) $50 \mu M$ de resveratrol (RES), $25 \mu \mathrm{M}$ de quercetina (QCT) $25 \mu \mathrm{M}$ de RES + $25 \mu \mathrm{M}$ de QCT o $50 \mu \mathrm{M}$ de RES + 25 $\mu \mathrm{M}$ de QCT, durante 24 horas. NOTA: las células fueron tratadas previamente a su estimulación durante $\mu \mathrm{M}$ de QCT, durante $\mathbf{2 4}$ horas. NOTA: las células fueron tratadas previamente a su estimulación durante con vehículo. ${ }^{*}: p<0,05$

40

Fig. 5. Muestra la cantidad de ciclooxigenasa 2 (COX-2), en unidades normalizadas, producida en células de epitelio corneal humano (HCE) estimuladas con $25 \mathrm{ng} / \mathrm{mL}$ de TNF- $\alpha$ y tratadas con vehículo $(0,5 \%$ de etanol (EtOH)), $50 \mu \mathrm{M}$ de resveratrol (RES), $25 \mu \mathrm{M}$ de quercetina (QCT), $25 \mu \mathrm{M}$ de RES + $25 \mu \mathrm{M}$ de QCT o $50 \mu \mathrm{M}$ de RES + $25 \mu \mathrm{M}$ de QCT, durante 24 horas. NOTA: las células fueron tratadas previamente a su estimulación durante 2 horas con el tratamiento correspondiente. Control células de HCE no estimuladas con TNF- $\alpha$ y tratadas con vehículo. *: $p<0,05$.

Fig. 6. Muestra la cantidad de fluorescencia en unidades normalizadas a la cantidad total de proteína de la sonda H2DCF-DA oxidada, como medida de la generación intracelular de especies proteina de la sonda H2DCF-DA oxidada, como medida de la generación intracelular de especies
relativas de oxígeno, en células de epitelio corneal humano (HCE) expuestas a radiación UVB y relativas de oxigeno, en con vehículo $(0,5 \%$ de etanol (EtOH)), $50 \mu \mathrm{M}$ de resveratrol (RES), $25 \mu \mathrm{M}$ de quercetina (QCT), $25 \mu M$ de RES + $25 \mu M$ de QCT o $50 \mu M$ de RES + $25 \mu M$ de QCT, durante una hora. NOTA: las células fueron tratadas previamente a su estimulación durante 1 hora con el tratamiento correspondiente. Control: células de HCE no irradiadas con UVB y tratadas con vehículo. *: p<0,05.

55

Fig. 7. Muestra el promedio de células T CD4 + en la conjuntiva de ratones C57BL/6 en un modelo de SOS en ratón (Dursun et al., 2002, Invest Ophthalmol Vis Sci.; 43(3):632-638) y tratados con vehículo, $0,01 \%$ resveratrol (RES), $0,01 \%$ quercetina (QCT) y $0,01 \%$ QCT + $0,01 \%$ RES. Control ratones no expuestos a ojo seco inducido (OSI) y no tratados tópicamente. Sin tratamiento: ratones 60 expuestos a OSI y no tratados tópicamente. *: $p<0,05$ 


\section{EJEMPLOS DE REALIZACIÓN}

A continuación se ilustrará la invención mediante unos ensayos realizados por los inventores, que ponen de manifiesto la efectividad de la composición de la invención, así como de la quercetina (QCT) y del 5 resveratrol (RES) individualmente, en la prevención y/o tratamiento de la inflamación, del estrés oxidativo y de la neovascularización ocular. Estos ejemplos específicos que se proporcionan sirven para ilustrar la y de la neovascularización ocular. Estos ejemplos específicos que se proporcionan sirven para ilustrar la ser interpretados como limitaciones a la invención que aquí se reivindica. Por tanto, los ejemplos descritos más adelante ilustran la invención sin limitar el campo de aplicación de la misma.

EJEMPLO 1. Efecto antiinflamatorio de la quercetina (QCT), del resveratrol (RES) y de su mezcla sobre una línea celular de epitelio corneal humano estimulada con TNF- $\alpha$

Para todos los experimentos se utilizó una línea celular (HCE) derivada de epitelio corneal humano. Las células fueron cultivadas en medio DMEM/F12 suplementado con $15 \%$ de suero fetal bovino (FBS), $0,5 \%$ de DMSO, $0,1 \mathrm{mg} / \mathrm{mL}$ de toxina colérica, $10 \mathrm{ng} / \mathrm{mL}$ de EGF, $5 \mathrm{mg} / \mathrm{mL}$ de insulina obtenida de páncreas bovino y antibióticos $(100 \mathrm{U} / \mathrm{mL}$ de penicilina y $0,1 \mathrm{mg} / \mathrm{mL}$ de estreptomicina). Para la realización de los 20 experimentos, se reemplazó el medio de cultivo por medio $\mathrm{DMEM}$ libre de $\mathrm{NaHCO}_{3}$, rojo fenol y piruvato.

RES, QCT y su mezcla se disolvieron en etanol $(\mathrm{EtOH})$. Las disoluciones stock fueron preparadas para tener una concentración constante de vehículo en todos los pocillos.

Las células se plantaron en placas de cultivo de 24 pocillos y se dejaron crecer hasta preconfluencia. Posteriormente, se pretrataron con $50 \mu \mathrm{M}$ de RES $25 \mu \mathrm{M}$ de QCT, $25 \mu \mathrm{M}$ de RES + $25 \mu \mathrm{M}$ de QCT o 50 $\mu \mathrm{M}$ de RES + $25 \mu \mathrm{M}$ de QCT, durante 2 horas. Pasado este tiempo, se eliminaron los sobrenadantes y se estimularon las células durante 24 horas con $25 \mathrm{ng} / \mathrm{mL}$ de TNF- $\alpha$ en presencia de RES, QCT o QCT+RES (se volvieron a ańadir). Como controles se utilizaron células no estimuladas con TNF- $\alpha$ y células estimuladas y tratadas ambas solo con vehículo. Una vez transcurrido el tiempo de incubación, los sobrenadantes se recogieron, se centrifugaron y se congelaron a $-80^{\circ} \mathrm{C}$ para su posterior análisis. Las células adheridas a las placas de cultivo también se congelaron a $-80^{\circ} \mathrm{C}$ para su posterior análisis.

35

\section{Determinación de la producción de citoquinas y quimioquinas}

Se llevó a cabo el análisis de la producción de las citoquinas IL-6 y VEGF y de las quimioquinas IL-8 e IP 10 en los sobrenadantes recogidos tras la estimulación con TNF-a. Este análisis se llevó a cabo mediante

40 tecnología X-MAP en un Luminex IS-100 con un ensayo multianalito comercial (Milliplex, Millipore) siguiendo las indicaciones del fabricante. Los valores de producción, en $\mathrm{pg} / \mathrm{mL}$, de citoquinas/quimioquinas (obtenidos tras la interpolación de los valores de fluorescencia en curvas total correspondiente en cada muestra, determinada mediante un ensayo de BCA comercial (Pierce, USA) en las células adheridas al pocillo

Determinación de la producción de ciclooxigenasa 2 (COX-2)

Para este ensayo se utilizó un ensayo comercial "Cell-based ELISA" (R\&D, USA) siguiendo las 50 indicaciones del fabricante. En este ensayo, se determinan simultáneamente las cantidades de COX-2 y de GAPDH de cada pocillo. Las células se plantaron en la placa del ensayo y se trataron y estimularon de la misma manera que se describió anteriormente. Finalizado el tiempo de incubación, se midieron los la misma manera que se describió anteriormente. Finalizado el tiempo de incubación, se midieron los valores de fluorescencia correspondientes a la cantidad de COX-2 y de GAPDH en un espectrofotómetro
(SpectraMAX M5, Molecular Devices, Inc. USA). Los valores de COX-2 en cada pocillo se normalizaron a

55 la cantidad de GAPDH correspondiente.

\section{Resultados de la producción de citoquinas y quimioquinas}

La figura 1 muestra la cantidad de IL-6 estimulada con TNF-a y su variación respecto a los tratamientos

60 con QCT, RES Y QCT RES. significativamente la producción de IL-6 $(p<0,05)$. QCT, RES y QCT+RES disminuyeron drásticamente los valores de IL-6 $(p<0,05)$. Los compuestos estudiados y su vehículo, en las concentraciones testadas, no fueron citotóxicos. 
La figura 2 muestra la cantidad de IL-8 estimulada con TNF- $\alpha$ y su variación respecto a los tratamientos con QCT, RES y QCT+RES. La estimulación de las células HCE con TNF- $\alpha$ aumentó significativamente la producción de IL-8 $(\mathrm{p}<0,05)$. QCT, RES y QCT+RES disminuyeron significativamente los valores de IL-8 La figura 3 muestra la cantidad de IP-10 estimulada con TNF-a y su variación respecto a los tratamientos con QCT, RES y QCT+RES. La estimulación de las células HCE con TNF- $\alpha$ aumentó significativamente la producción de IP-10 (p<0,05). QCT, RES y QCT+RES disminuyeron significativamente los valores de IP10 hasta niveles basales $(p<0,05)$.

10

La figura 4 muestra la cantidad de VEGF estimulada con TNF- $\alpha$ y su variación respecto a los tratamientos con QCT, RES y QCT+RES. La estimulación de las células HCE con TNF- $\alpha$ aumentó significativamente la producción de VEGF $(p<0,05)$. QCT, RES y QCT+RES disminuyeron significativamente los valores de VEGF hasta niveles basales $(p<0,05)$

Resultados de la producción de COX-2

La figura 5 muestra la cantidad total de COX-2 (normalizada con GAPDH) estimulada con TNF- $\alpha$ y su variación respecto a los tratamientos con QCT. RES y QCT+RES. La estimulación de las células HCE con

20 TNF- $\alpha$ aumentó significativamente la producción de COX-2 $(\mathrm{p}<0,05)$. QCT. RES y QCT+RES disminuyeron significativamente la cantidad de COX-2 estimulada por TNF- $\alpha(p<0,05)$.

EJEMPLO 2. Efecto antioxidante del resveratrol (RES), de la quercetina (QCT) y de su mezcla sobre una línea celular de epitelio corneal humano estimulada con radiación ultravioleta.

25

Cultivo celular

Como en el ejemplo anterior, se utilizó la línea celular HCE derivada de epitelio corneal humano. Las células fueron cultivadas en medio DMEM/F 12 suplementado con $15 \%$ de suero fetal bovino (FBS), $0,5 \%$ de DMSO, $0,1 \mathrm{mg} / \mathrm{mL}$ de toxina colérica, $10 \mathrm{ng} / \mathrm{mL}$ de EGF, $5 \mathrm{mg} / \mathrm{mL}$ de insulina obtenida de páncreas de DMSO, $0,1 \mathrm{mg} / \mathrm{mL}$ de toxina colerica, $10 \mathrm{ng} / \mathrm{mL}$ de EGF, $5 \mathrm{mg} / \mathrm{mL}$ de insulina obtenida de pancreas
bovino y antibióticos (100 $\mathrm{U} / \mathrm{mL}$ de penicilina y $0,1 \mathrm{mg} / \mathrm{mL}$ de estreptomicina). Para la realización de los experimentos, se reemplazó el medio de cultivo por medio $\mathrm{DMEM}$ libre de $\mathrm{NaHCO}_{3}$, rojo fenol y piruvato.

QCT, RES y su mezcla se disolvieron en etanol ( $\mathrm{EtOH})$. Las disoluciones stock fueron preparadas para tener una concentración constante de vehículo en todos los pocillos.

\section{Estrés oxidativo intracelular provocado por estimulación con luz ultravioleta}

Las células se plantaron en placas de cultivo de 24 pocillos y se dejaron crecer hasta preconfluencia 40 Posteriormente, se pretrataron con $50 \mu \mathrm{M}$ de RES, $25 \mu \mathrm{M}$ de QCT, $25 \mu \mathrm{M}$ de RES + $25 \mu \mathrm{M}$ de QCT o 50 $\mu \mathrm{M}$ de RES + $25 \mu \mathrm{M}$ de QCT, durante 1 hora. Seguidamente, se cargaron las células con $10 \mu \mathrm{M}$ de diacetato de 2',7'-diclorodihidrofluoresceina (H2DCF-DA) durante 30 minutos. A continuación, se trataron las células con QCT, RES o QCT+RES, se expusieron a luz UVB (302 nm; $\left.107 \mathrm{~mJ} / \mathrm{cm}^{2}\right)$ y se dejaron en incubación a $37^{\circ} \mathrm{C}$ durante 1 hora Como controles se utilizaron células no irradiadas y células irradiadas,

45 y tratadas ambas solo con vehículo. Finalmente, se leyó la fluorescencia a $522 \mathrm{~nm}$. Los valores de fluorescencia se normalizaron respecto a la cantidad de proteína total en cada muestra, determinada mediante un ensayo de BCA comercial (Pierce, USA) en las células adheridas al pocillo.

Resultados del estrés oxidativo provocado por radiación UVB

La figura 6 muestra la cantidad de fluorescencia relativa producida por la exposición a la radiación UVB y su variación respecto a los tratamientos con QCT, RES y QCT+RES. La estimulación de las células HCE con radiación UVB produjo un aumento significativo de especies oxidativas $(0<0,05)$. QCT. RES y QCT+RES actuaron como antioxidantes disminuyendo los niveles de especies oxidativas generadas por 55 la exposición a la radiación UVB, disminución que en el caso de la QCT y de la QCT+RES fue significativa $(p<0,05)$.

EJEMPLO 3. Efecto antiinflamatorio de la quercetina (QCT), del resveratrol (RES) y de su mezcla administrados en forma tópica, en un modelo animal de ojo seco.

60

Modelo animal de ojo seco 
El ojo seco inducido (OSI) se provocó en ratones C57BL/6 mediante la inyección subcutánea de $200 \mu \mathrm{L}$ de escopolamina $(5 \mathrm{mg} / \mathrm{mL})$ tres veces al día durante 10 días, y la exposición a un flujo de aire constante durante 24 horas. La humedad en la sala se mantuvo entre el $30 \%$ y $35 \%$, a una temperatura constante de $25^{\circ} \mathrm{C}$ (Dursun et al. Invest Ophthalmol Vis Sci. 2002; 43(3):632-8).

\section{Ratones y tratamientos tópicos}

Los ratones C57BL/ 6 se dividieron en 6 grupos de acuerdo con el tratamiento seguido:

a) Grupo control: ratones no expuestos a OSI y no tratados tópicamente.

b) Grupo OSI: ratones expuestos a OSI y no tratados tópicamente.

c) Grupo OSI+vehículo: ratones expuestos a OSI y tratados tópicamente con el vehículo.

d) Grupo OSI+RES: ratones expuestos a OSI y tratados tópicamente con $0,01 \%$ RES

e) Grupo OSI+QCT: ratones expuestos a OSI y tratados tópicamente con $0,01 \%$ QCT

f) Grupo OSI+QCT+RES: ratones expuestos a OSI y tratados tópicamente con $0.01 \%$ QCT G,01\% RES

Los tratamientos tópicos comenzaron 1 día antes de inducir el ojo seco, instilando en ambos ojos $5 \mu \mathrm{L}$ de tratamiento correspondiente para cada grupo tres veces al día.

20

Determinación de linfocitos T CD4+ en conjuntiva

Los ojos embebidos en OCT se seccionaron en un criostato Las muestras se fijaron con acetona fría a $20 \stackrel{\circ}{\circ}$. Posteriormente, se bloqueó la peroxidasa endógena con peróxido de hidrógeno. Después, se bloquearon las uniones no específicas con suero y se incubaron las muestras con el anticuerpo primario a temperatura ambiente. Consecutivamente, se utilizó un anticuerpo secundario biotinilado seguido de reactivo ABC. Finalmente, se utilizó el cromógeno vector NovaRed para colorear el marcaje de la IMQ, seguido de contratinción nuclear con hematoxilina. Se examinaron tres secciones de cada ratón a microscopio óptico y los resultados se expresaron como el promedio de células T CD4+ en conjuntiva.

30 Resultados de las propiedades antiinflamatorias de QCT, RES y su mezcla en un modelo animal de ojo seco

La figura 7 muestra el promedio de linfocitos $T$ CD4+ en conjuntiva respecto a los tratamientos tópicos con vehículo, QCT, RES y QCT+RES. El modelo de ojo seco produjo un aumento en la cantidad de células

$35 \mathrm{CD} 4+$ en conjuntiva respecto al grupo control $(p<0,05)$. RES mostró una disminución no significativa ( $p>0,05)$ de linfocitos CD4+, comparada con el grupo OSI+vehículo. QCT y QCT+RES disminuyeron significativamente la cantidad de células CD4+ en conjuntiva producida por OSI en ratones, comparado con el grupo OSI+vehículo $(p<0,05)$.

40 


\section{REIVINDICACIONES}

1. Composición que comprende quercetina, o cualquiera de sus sales o derivados, y al menos otro compuesto estilbenoide, o cualquiera de sus sales o derivados.

2. Composición según la reivindicación 1 donde el otro compuesto estilbenoide es resveratrol, o cualquiera de sus sales o derivados

103 . Composición según cualquiera de las reivindicaciones 162 que además comprende un vehículo farmacéuticamente aceptable.

4. Uso de la composición según cualquiera de las reivindicaciones 1 a 3 para la elaboración de un medicamento.

15

5. Uso de la composición según la reivindicación 4 donde el medicamento está formulado para su administración oftálmica.

6. Uso de la composición según cualquiera de las reivindicaciones 4 ó 5 donde el medicamento es para el tratamiento y/o prevención de la inflamación ocular.

7. Uso de la composición según la reivindicación 6 donde el medicamento es para el tratamiento y/o prevención de la inflamación del segmento anterior y/o de la glándula lagrimal principal.

8. Uso de la composición según la reivindicación 7 donde el medicamento es para el tratamiento y/o prevención de la inflamación de la superficie ocular.

9. Uso de la composición según cualquiera de las reivindicaciones 465 donde el medicamento es para el tratamiento y/o prevención del estrés oxidativo ocular.

10. Uso de la composición según la reivindicación 9 donde el medicamento es para el tratamiento y/o prevención del estrés oxidativo del segmento anterior.

11. Uso de la composición según la reivindicación 10 donde el medicamento es para el tratamiento y/o prevención del estrés oxidativo de la superficie ocular.

12. Uso de la composición según cualquiera de las reivindicaciones 4 ó 5 donde el medicamento es para el tratamiento y/o prevención de la neovascularización ocular

13. Uso de la composición según la reivindicación 12 donde el medicamento es para el tratamiento y/o prevención de la neovascularización del segmento anterior.

14. Uso de la composición según la reivindicación 13 donde el medicamento es para el tratamiento y/o prevención de la neovascularización de la córnea.

15. Uso de la composición según cualquiera de las reivindicaciones 6 a 14 donde el medicamento es para el tratamiento y/o prevención de síndrome de ojo seco, blefaritis, disfunción de las glándulas de Meibomio, meibomitis, procesos alérgicos oculares, distrofias corneales, conjuntivitis, alteración de la superficie ocular por el uso de lentes de contacto, enfermedades autoinmunes que afecten a

50 segmento anterior del ojo, postcirugía del segmento anterior, quemaduras o lesiones o patologías oculares producidas por radiación ultravioleta. 





FIG. 2

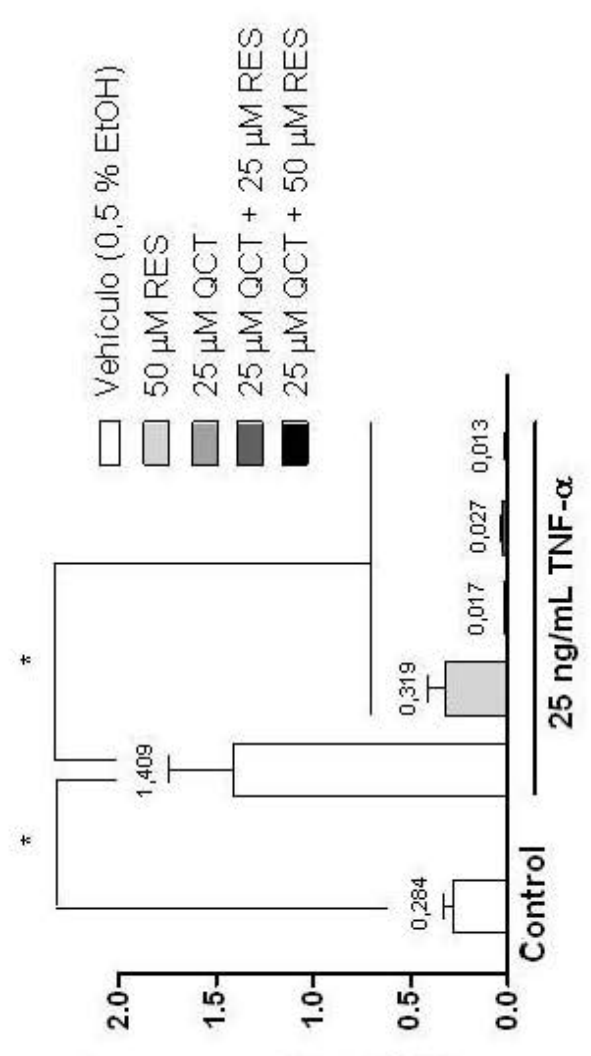

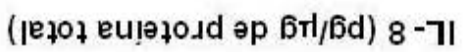


FIG. 3

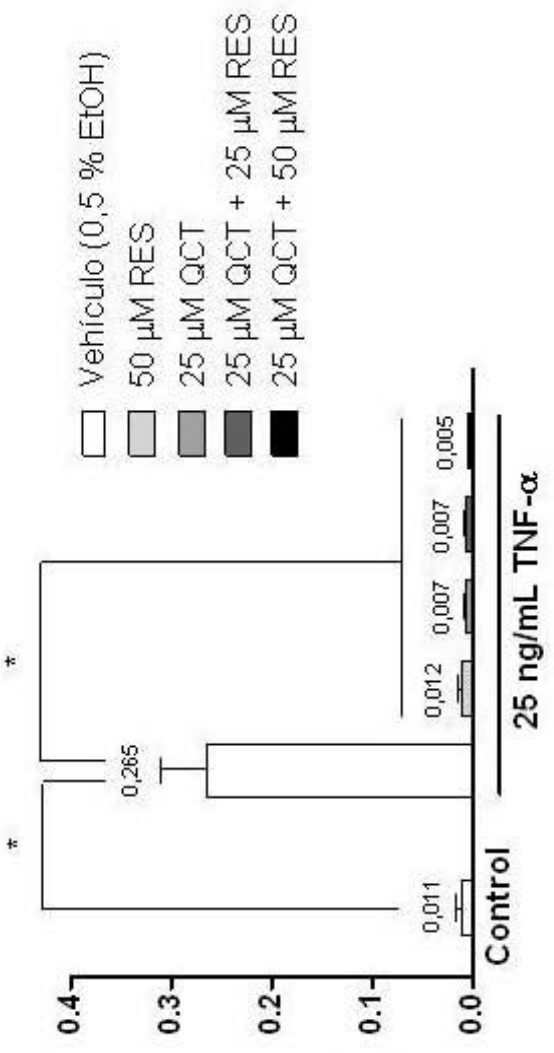

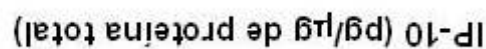


FIG. 4

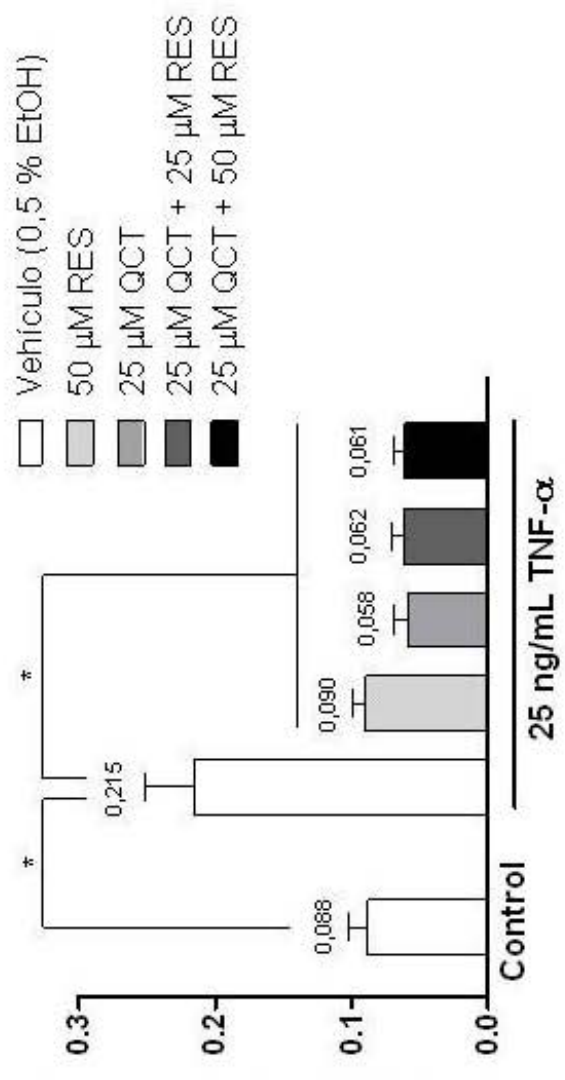

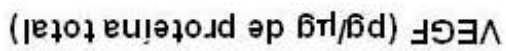


FIG. 5
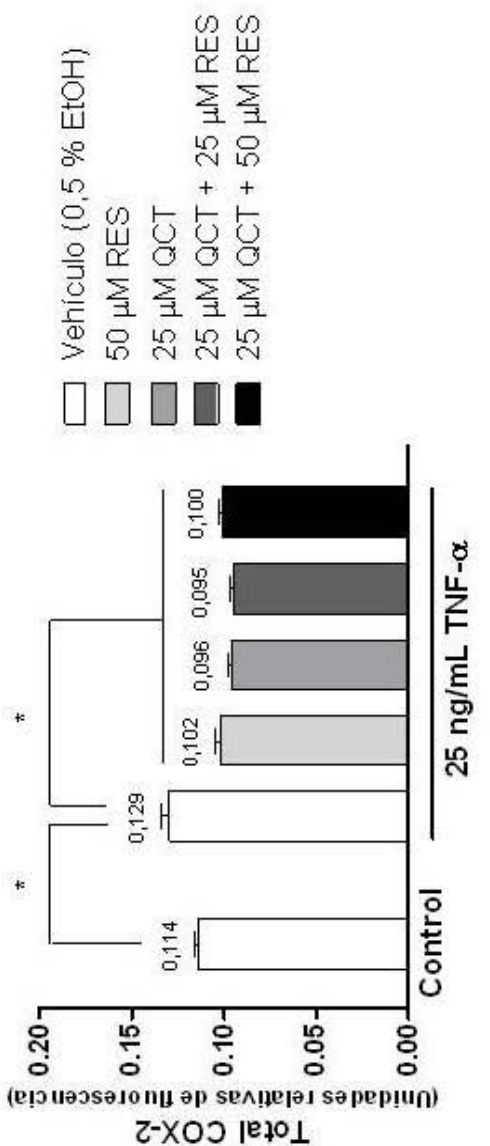
FIG. 6

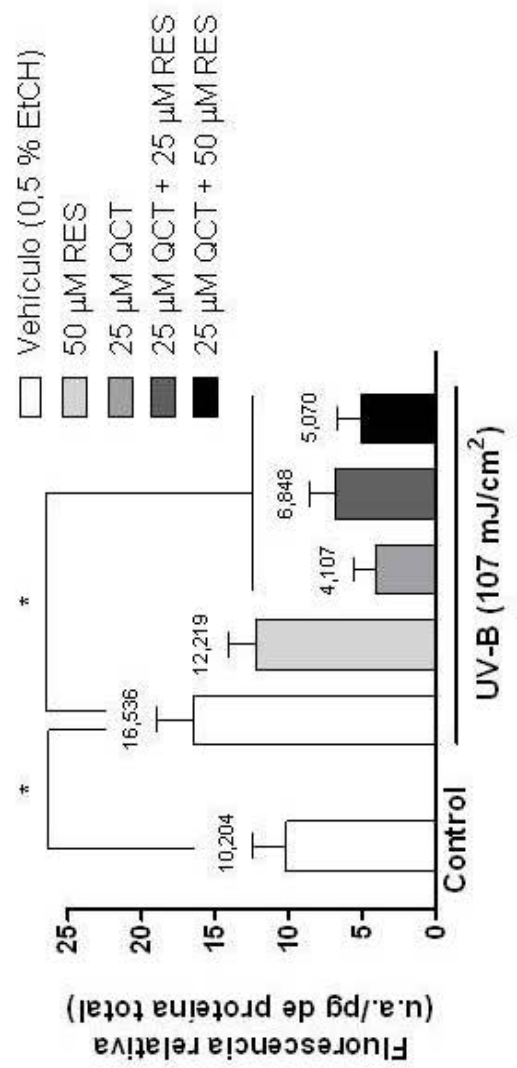

20 
FIG. 7
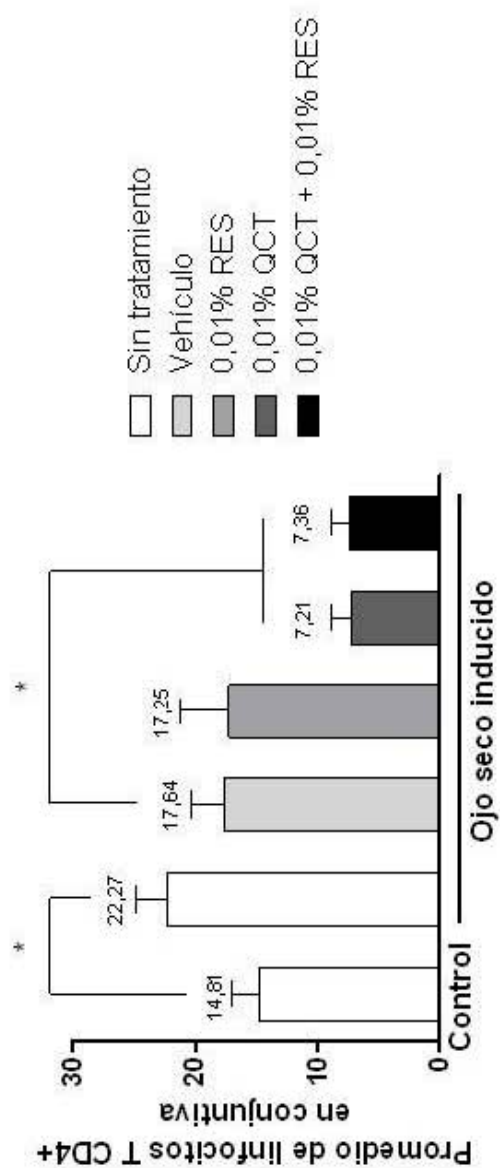


\section{RESUMEN}

COMPOSICIÓN PARA SU USO EN EL TRATAMIENTO Y/O PREVENCIÓN DE LA INFLAMACIÓN, EL ESTRÉS OXIDATIVO Y LA NEOVASCULARIZACIÓN OCULAR.

La presente invención se refiere al uso de la quercetina (QCT) o de una composición que comprende QCT y al menos otro compuesto polifenólico, preferiblemente resveratrol (RES), para el tratamiento y/o prevención de lesiones o enfermedades que cursan con inflamación, estrés oxidativo y/o neovascularización ocular, preferiblemente del segmento anterior, más preferiblemente de la superficie ocular y aun más preferiblemente del epitelio corneal. La invención también se refiere al uso de la QCT y de dicha composición como agente antimicrobiano, antioxidante y antiinflamatorio en soluciones de limpieza y mantenimiento de lentes de contacto. 

XIII. Appendix II 



\section{A preliminary study of the effect of QCT and RES on a normal conjunctival epithelial cell line exposed to UV-B radiation}

This appendix describes briefly the results from a preliminary study regarding the effect of QCT and RES in UV-B-induced apoptosis in IOBA-NHC cells. These experiments were carried out in Allergan headquarter (Irvine, CA, USA) under the supervision of Dr. Michael E. Stern in 2013. This section has been divided into five parts: I) brief introduction describing the molecular mechanism underlying apoptosis, II) methodology, III) results and IV) conclusions.

\section{Introduction}

Apoptosis is the process of programmed cell death that occurs in autoimmune and inflammatory diseases. DED is characterised by an increased apoptotic cell death in the ocular resident epithelium of the LFU, ${ }^{83}$ suggesting that this process contributes to pathogenesis of DED.

Apoptosis can be triggered by an extrinsic mechanism mediated by death receptors (members of the TNF receptor gene superfamily) located in the outer membrane of cells, or by an intrinsic mechanism mediated by mitochondria.

Focusing on the intrinsic mechanism, this is an early apoptotic process that involves an increase of the outer mitochondrial membrane permeability that results in the loss of mitochondrial membrane potential $(\Delta \Psi)$, finally leading to rupture of the outer mitochondrial membrane. This process is called the mitochondrial membrane permeability transition (MPT). Thus, the MPT refers to the massive swelling and depolarisation of mitochondria that occurs under some conditions, most notably as a result of calcium overload and oxidative stress. The cause of the MPT is the opening of a non-specific pore in the inner mitochondrial membrane, known as the mitochondrial permeability transition pore (MPTP) ${ }^{390}$ As a result, proteins from the intermembrane space are released into the cytoplasm, including apoptogenic molecules such as cytochrome $\mathrm{C}$ or Smac/Diablo, triggering a cascade of caspase activation that propagates apoptotic signals. ${ }^{391}$

A previous experiment from this thesis demonstrated that UV-B increases oxidative stress and QCT and RES can reduce UV-B-induced ROS in IOBA-NHC cells. UV-B radiation is also a pro-inflammatory stimulus ${ }^{233,280}$ that can cause apoptosis. ${ }^{392}$

It has been described that inflammatory stimuli such as TNF- $\alpha$ and IFN- $\gamma$ produces aberrant MPTP opening resulting in an increased apoptosis in IOBA-NHC cells. ${ }^{393}$ Thus, the aim of this work was to investigate the role of MPTP and the effect of 
QCT and RES on inflammatory apoptosis of human conjunctival epithelial cells exposed to UV-B radiation.

\section{Materials and methods}

\section{$\underline{\text { Cell line and culture conditions }}$}

IOBA-NHC cells were used for this experiment. Cells were culture as described in section V.1.3. All experiments were carried out using the CM also described in section V.1.3.

\section{$\underline{U V-B \text { exposure }}$}

Cells were seeded in $75 \mathrm{~cm}^{2}$ flasks at $2 \times 10^{6}$ cells per flask to confluence. Then, culture medium was replaced with $\mathrm{CM}$ and cells were exposed to UV-B light $(9.5$ $\mathrm{mW} / \mathrm{cm}^{2}$ ) for $1 \mathrm{~min}$. After light exposure, cells were cultured for $1 \mathrm{~h}$ and then collected by trypsin for further experiments.

\section{Polyphenol treatments}

QCT and RES were dissolved in EtOH. Fresh stock solutions of QCT and RES were firstly prepared and serial dilutions were carried out to achieve final concentrations of 1, 10 and $25 \mu \mathrm{M}$ QCT; 5, 50 and $100 \mu \mathrm{M}$ RES; and $0.5 \% \mathrm{EtOH}$ (vehicle). Polyphenol treatments were added $1 \mathrm{~h}$ before UV-B.

\section{Detection of MPTP opening}

MPTP opening was determined using the MitoProbe ${ }^{\mathrm{TM}}$ Transition Pore Assay Kit (Life Technologies), as previously described by Gao et al. ${ }^{393}$ Briefly, UV-B-exposed cells were collected and then washed with PBS without $\mathrm{MgCl}_{2}$ and $\mathrm{CaCl}_{2}$. After that, cells were loaded with $2 \mu \mathrm{M}$ calcein acetoxymethyl (AM) and $80 \mathrm{mM} \mathrm{CoCl}_{2}$. Ionomycin (10 $\mathrm{nM})$, an inductor of apoptosis, was added as a positive control. Cells were then incubated for $15 \mathrm{~min}$, washed and analysed using a flow cytometer (BD).

Calcein AM is a fluorescent dye that passively diffuses into cells. Upon entering the cells, intracellular esterases cleave the acetoxymethyl (AM) ester group, yielding the membrane-impermeable calcein fluorescent dye. In the absence of $\mathrm{CoCl}_{2}$ and ionomycin, fluorescent calcein is present in the cytosol as well as the mitochondria, resulting in a bright signal. In the presence of $\mathrm{CoCl}_{2}$, calcein in the mitochondria emits a signal, but the cytosolic calcein fluorescence is quenched; the overall fluorescence is reduced compared to calcein alone. When ionomycin (a calcium ionophore) and $\mathrm{CoCl}_{2}$ 
are added to cells at the same time as calcein AM, the fluorescence signals from both cytosol and mitochondria are largely abolished.

\section{$\underline{\text { Apoptosis }}$}

Apoptosis was determined using a FITC annexin V apoptosis detection kit (BD), following manufacturer's instructions. Briefly, collected UV-B-exposed cells were washed with PBS and resuspended in $1 \mathrm{X}$ binding buffer at $10^{6}$ cells $/ \mathrm{mL}$. Then, $5 \mu \mathrm{L}$ of FITC Annexin V and $5 \mu \mathrm{L} \mathrm{PI}$ were added to $100 \mu \mathrm{L}$ of cell suspension and incubated for $15 \mathrm{~min}$ at room temperature in the dark. Finally, $400 \mu \mathrm{L}$ of $1 \mathrm{X}$ binding buffer were added and analysed by flow cytometry. Percentage of positive annexin $\mathrm{V}$ and positive PI cells were obtained using the CellQuest Pro software (BD).

\section{Statistical analysis}

All data were expressed as mean \pm SEM. Statistics were analysed using the SPSS software package (SPSS version 15.0 for Windows, SPSS Inc., Chicago, IL, USA). Homogeneity of variances were analysed using Levene's test. Data were analysed using the $t$-test or $t$-test with Welch correction. Two different experiments were performed in duplicate.

\section{Results}

Figure 1 shows the effect of QCT and RES on MPTP opening (Figure 1A) and late apoptosis (Figure 1B) in IOBA-NHC cells. MPTP opening was induced in the normal IOBA-NHC cells by ionomycin $(p<0.001)$ as a positive control. UV-B radiation (570 m J/cm2) induced MPTP opening in IOBA-NHC cells after $1 \mathrm{~h}$. QCT at 1, 10 and $25 \mu \mathrm{M}$ (all $\mathrm{p}<0.01)$ doses and RES at $5 \mu \mathrm{M}(\mathrm{p}<0.01)$ and $50 \mu \mathrm{M}(\mathrm{p}<0.05)$ doses significantly inhibited MPTP opening (Figure 1A) without affecting apoptosis (Figure 1B). 
A



B

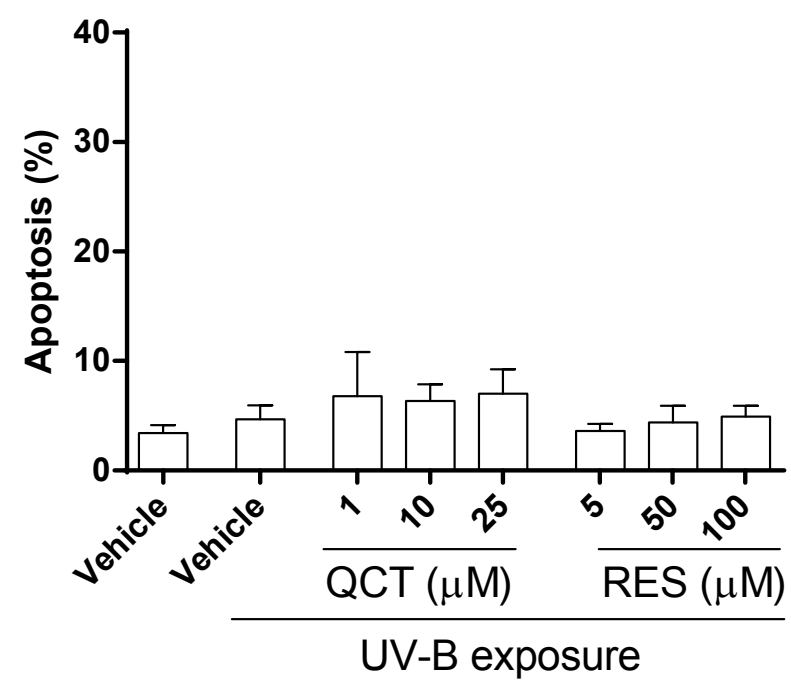

Figure 1. Effect of quercetin (QCT) and resveratrol (RES) on MPTP (A) and late apoptosis (B) on UV-B-exposed IOBA-NHC cell. ${ }^{* *} p<0.01,{ }^{* * *} p<0.001$, compared to unexposed cells treated with vehicle; ${ }^{+} p<0.05,{ }^{++} p<0.01$, compared to vehicle-treated UV-B-exposed cells $(0.5 \% \mathrm{EtOH}) . \mathrm{N}=2$ for both experiments.

\section{Conclusions}

a) UV-B radiation $\left(570 \mathrm{~mJ} / \mathrm{cm}^{2}\right)$ promoted MPTP opening in IOBA-NHC cells.

b) UV-B radiation $\left(570 \mathrm{~mJ} / \mathrm{cm}^{2}\right)$ did not induce apoptosis after $1 \mathrm{~h}$.

c) QCT prevented MPTP opening at all concentrations tested (1, 10 and $25 \mu \mathrm{M})$ in UV-B-exposed IOBA-NHC cells and did not induce apoptosis.

d) Concentrations of 5 and $50 \mu \mathrm{M}$ RES prevented MPTP opening, without affecting apoptosis. 
XIV. Appendix III 

$+b$

COMITÉ ÉTICO DE INVESTIGACIÓN CLÍNICA

ÁREA DE SALUD VALLADOLID - ESTE (CEIC-VA-ESTE-HCUV)

\section{Sacyl}

Valladolid a 23 de Mayo de 2013

En la reunión del CEIC ÁREA DE SALUD VALLADOLID - ESTE del 23 de Mayo de 2013 , se procedió a la evaluación de los aspectos éticos del siguiente proyecto de investigación

A continuación les señalo los acuerdos tomados por el CEIC ÁREA DE SALUD VALLADOLID - ESTE en relación a dicho Proyecto de Investigación:

\begin{tabular}{|l|l|l|}
\hline PI-13-82 & "EFECTOS DE COMPUESTOS CXCLL-1 Y & IOBA \\
& CXCLL-2 SOBRE CÉLULAS & Investigador principal: \\
& MONONUCLEARES PROCEDENTES DE & Dra. AMALIA ENRIQUEZ \\
& SANGRE PEROFÉRICA HUMANA" & DE SALAMANCA \\
& (BIOQUIMICA) \\
& Equipo investigador: \\
& ANTONIO ABENGÓZAR \\
& VELA (QUÍMICO, ÓPTICO- \\
& OPTOMETRISTA), \\
& CARMEN GARCÍA \\
& VÁZQUEZ (TÉCNICO DE \\
& LABORATORIO) Y Ma \\
& JESUS GONZÁLEZ \\
& GARCÍA \\
& (ÓPTICO.OPTOMETRISTA) \\
& Recibido: 07-05-2013 \\
\hline
\end{tabular}

Considerando que el Proyecto contempla los Convenios y Normas establecidos en la legislación española en el ámbito de la investigación biomédica, la protección de datos de carácter personal y la bioética, se hace constar el informe favorable y la aceptación del Comité Ético de Investigación Clínica del Área de Salud Valladolid Este para que sea llevado a efecto dicho Proyecto de Investigación.

Un cordial saludo.

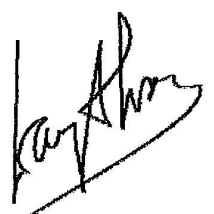

T. $\tan$ Alueng



Dr. F. Javier Álvarez.

CEIC Área de Salud Valladolid Este - Hospital Clínico Universitario de Valladolid

Farmacología

Facultad de Medicina,

Universidad de Valladolid,

c/ Ramón y Cajal 7

47005 Valladolid

alvarez@med.uva.es

jalvarezgo@saludcastillayleon.es

tel: 983423077



Castilla y teo de 


\section{HOJA DE INFORMACIÓN AL PACIENTE Y CONSENTIMIENTO INFORMADO}

\section{Título del estudio:}

Efecto de los compuestos CXCLL-1 y CXCLL-2 sobre células mononucleares procedentes de sangre periférica humana.

\section{Investigador principal: Amalia Enríquez de Salamanca Aladro}

\section{Centro: IOBA, Universidad de Valladolid}

\section{Introducción.}

Nos dirigimos a usted para informarle sobre el desarrollo del estudio en el que se le propone participar. Su participación en el estudio consiste en donar una muestra de sangre para aislar células mononucleares (linfocitos $\mathrm{T}$ ) y estudiar sobre ellas las propiedades antiinflamatorias de ciertos compuestos de origen natural.

Nuestra intención es que usted reciba la información correcta y suficiente para que pueda evaluar y juzgar si quiere o no participar en este estudio. Para ello, lea esta hoja informativa con atención y nosotros le aclararemos las dudas que le puedan surgir después de la explicación.

\section{Objetivos y justificación del estudio.}

El síndrome de ojo seco es una enfermedad inflamatoria de la superficie ocular que afecta aproximadamente al $15 \%$ de la población mundial. Uno de los problemas de la terapia médica es la falta de tratamientos efectivos para el síndrome de ojo seco; siendo el uso de lágrimas artificiales para reducir la sintomatología el único tratamiento hasta el momento disponible en España.

El objetivo final del estudio es desarrollar un tratamiento efectivo basado en compuestos de origen natural para el síndrome de ojo seco. Para ello, se pretende estudiar si los compuestos naturales objeto de estudio son capaces de disminuir la proliferación y el tipo de células mononucleares estimuladas en experimentos in vitro.

\section{Participación voluntaria.}

Debe saber que su participación en este estudio es voluntaria y que puede decidir no participar o cambiar su decisión y retirar el consentimiento en cualquier momento, sin que por ello se altere la relación con el centro. En caso de retirar el consentimiento para participar en el estudio, ningún dato nuevo será añadido a la base de datos y puede exigir la destrucción de todas las muestras identificables previamente retenidas para evitar la realización de nuevos análisis.

\section{Descripción general del estudio. Procedimiento.}

Para este procedimiento no se le administrará tratamiento extraordinario alguno. Si acepta la participación mediante la firma de este documento, se le realizará una extracción de sangre venosa de aproximadamente $40 \mathrm{~mL}$ (volumen máximo). La sangre se procesará para aislar las células mononucleares y éstas se utilizarán para determinar el efecto de los compuestos objeto de estudio, que tienen propiedades antiinflamatorias. 
Usted deberá notificar al responsable del estudio si en el momento de la misma padece alguna enfermedad (muy especialmente si padece de algún tipo de alergia o proceso inflamatorio crónico) y/o está tomando algún tipo de medicación, bien sea bajo prescripción o no.

En el caso de requerirse una nueva extracción por imposibilidad de obtener los datos necesarios de la muestra ya extraída para la realización del estudio, se le requerirá para que realice una visita para realizar otra extracción de sangre.

\section{Manejo de las muestras.}

Las muestras obtenidas no se etiquetarán con ningún dato que permita revelar la identidad del donante. Las muestras obtenidas se recogerán en diferentes tipos de tubos para su procesamiento y serán destruidas una vez terminen los experimentos.

\section{Confidencialidad.}

Los datos recogidos para el estudio estarán identificados mediante un código y solo el investigador principal del estudio, y sus colaboradores, podrán relacionar dichos datos con usted. Por lo tanto, su identidad no será revelada a persona alguna ajena a los procedimientos aquí descritos.

Todos los datos recogidos para el estudio, procedentes de su Historia Clínica o facilitados por usted mismo, serán tratados con las medidas de seguridad establecidas en cumplimiento de la Ley Orgánica 15/1999 de Protección de Datos de carácter personal. Debe saber que tiene derecho de acceso, rectificación y cancelación de los mismos en cualquier momento. Los datos recogidos para el estudio estarán identificados mediante un código y solo el investigador principal/colaboradores podrán relacionar dichos datos con usted y con su historia clínica.

Todas las muestras obtenidas serán utilizadas exclusivamente para los fines descritos en este documento y serán destruidas al finalizar el estudio.

\section{Otra información relevante.}

Si usted decide retirar el consentimiento para participar en este estudio, ningún dato nuevo será añadido a la base de datos $\mathrm{y}$, puede exigir la destrucción de todas las muestras identificables previamente retenidas para evitar la realización de nuevos análisis. También debe saber que puede ser excluido del programa si los responsables del estudio lo consideran oportuno.

En caso de necesitar cualquier información o por cualquier otro motivo no dude en contactar con los investigadores principales del estudio en el teléfono 983184761.

\section{Riesgos.}

La extracción de sangre tiene como principal inconveniente la percepción de una molestia mínima derivada de la extracción de sangre. Muy infrecuentemente se pueden producir un hematoma leve, o una hemorragia leve local.

Dado a que no es esperable que aparezcan acontecimientos adversos en lo que concierne a los sujetos que participan en la investigación, no se han tomado especiales medidas orientadas a su tratamiento. 
CONSENTIMIENTO INFORMADO: Efecto de los compuestos CXCLL-1 y CXCLL-2 sobre células mononucleares procedentes de sangre periférica humana.

Promotor: IOBA

Responsables del proyecto: Amalia Enríquez de Salamanca Aladro, Instituto de Oftalmobiología Aplicada (IOBA), Paseo de Belén 17; 47011 Valladolid.

Centro donde se realiza la recogida de muestra:

Yo, (nombre y apellidos):

He leído la Hoja de Información que se me ha entregado, he podido hacer preguntas sobre el Programa y he recibido suficiente información sobre el estudio.

He hablado con, (nombre y apellidos del investigador):

Comprendo que mi participación es voluntaria y que puedo retirarme del estudio cuando quiera, sin tener que dar explicaciones y sin que esto repercuta en mis cuidados médicos.

Presto libremente mi conformidad para participar en el proyecto y doy mi consentimiento para el acceso y utilización de mis datos en las condiciones detalladas en la hoja de información.

\begin{tabular}{|l|l|}
\hline Firma del paciente: & Firma del investigador: \\
\hline Nombre: & \\
\hline Fecha: & Nombre: \\
\hline
\end{tabular}

Copia para el paciente 
CONSENTIMIENTO INFORMADO: Efecto de los compuestos CXCLL-1 y CXCLL-2 sobre células mononucleares procedentes de sangre periférica humana.

Promotor: IOBA

Responsables del proyecto: Amalia Enríquez de Salamanca Aladro, Instituto de Oftalmobiología Aplicada (IOBA), Paseo de Belén 17; 47011 Valladolid.

Centro donde se realiza la recogida de muestra:

Yo, (nombre y apellidos):

He leído la Hoja de Información que se me ha entregado, he podido hacer preguntas sobre el Programa y he recibido suficiente información sobre el estudio.

He hablado con, (nombre y apellidos del investigador):

Comprendo que mi participación es voluntaria y que puedo retirarme del estudio cuando quiera, sin tener que dar explicaciones y sin que esto repercuta en mis cuidados médicos.

Presto libremente mi conformidad para participar en el proyecto y doy mi consentimiento para el acceso y utilización de mis datos en las condiciones detalladas en la hoja de información.

\begin{tabular}{|l|l|}
\hline Firma del paciente: & Firma del investigador: \\
\hline Nombre: & \\
\hline Fecha: & Nombre: \\
\hline
\end{tabular}

Copia para el centro 

References 

1. Ward SK, Wakamatsu TH, Dogru M, et al. The role of oxidative stress and inflammation in conjunctivochalasis. Invest.Ophthalmol.Vis.Sci. 2010;51:19942002.

2. Bacsi A, Dharajiya N, Choudhury BK, Sur $S$, Boldogh I. Effect of pollen-mediated oxidative stress on immediate hypersensitivity reactions and late-phase inflammation in allergic conjunctivitis. J.Allergy Clin.Immunol. 2005;116:836-843.

3. Wakamatsu TH, Dogru M, Matsumoto $\mathrm{Y}$, et al. Evaluation of lipid oxidative stress status in sjogren syndrome patients. Invest. Ophthalmol.Vis.Sci. $\quad 2013 ; 54: 201-$ 210.

4. Stern ME, Beuerman RW, Fox RI, Gao J, Mircheff AK, Pflugfelder SC. The pathology of dry eye: The interaction between the ocular surface and lacrimal glands. Cornea 1998;17:584-589.

5. Stern ME, Beuerman RW, Pflugfelder SC. The normal tear film and ocular surface. S. C. Pflugfelder, R. W. Beuerman and M. E. Stern. In Dry eye and the ocular surface. New York: Marcel-Dekker; 2004. 41-62 pp.

6. Beuerman RW, Mircheff A, Pflugfelder SC, Stern ME. The lacrimal functional unit. S. C. Pflugfelder, R. W. Beuerman and M. E. Stern. In Dry eye and the ocular surface. New York: Marcel Dekker; 2004. 11-39 pp.

7. Nichols BA, Chiappino ML, Dawson CR. Demonstration of the mucous layer of the tear film by electron microscopy. Invest.Ophthalmol.Vis.Sci. $\quad$ 1985;26:464473.

8. Prydal Jl, Artal P, Woon H, Campbell FW. Study of human precorneal tear film thickness and structure using laser interferometry. Invest.Ophthalmol.Vis. Sci. 1992;33:2006-2011.

9. Dilly PN. Structure and function of the tear film. Adv.Exp.Med.Biol. 1994;350:239247.

10. Pflugfelder SC, Solomon A, Stern ME. The diagnosis and management of dry eye: A twenty-five-year review. Cornea 2000;19:644-649.
11. Green-Church KB, Nichols KK, Kleinholz NM, Zhang L, Nichols JJ. Investigation of the human tear film proteome using multiple proteomic approaches. Mol.Vis. 2008;14:456-470.

12. Dartt DA. Tear lipocalin: Structure and function. Ocul.Surf. 2011;9:126-138.

13. Flanagan JL, Willcox MD. Role of lactoferrin in the tear film. Biochimie 2009;91:35-43.

14. Sack RA, Conradi L, Krumholz D, Beaton A, Sathe S, Morris C. Membrane array characterization of 80 chemokines, cytokines, and growth factors in open- and closed-eye tears: Angiogenin and other defense system constituents. Invest. Ophthalmol.Vis.Sci. $\quad$ 2005;46:12281238.

15. Garreis F, Gottschalt M, Paulsen FP. Antimicrobial peptides as a major part of the innate immune defense at the ocular surface. Dev. Ophthalmol. 2010;45:16-22.

16. Watanabe $\mathrm{H}$. Significance of mucin on the ocular surface. Cornea 2002;21:S17-22.

17. Govindarajan B, Gipson IK. Membranetethered mucins have multiple functions on the ocular surface. Exp.Eye Res. 2010;90:655-663.

18. Calonge M, Stern ME. The conjunctiva and tear film maintenance. S. C. Pflugfelder, R. W. Beuerman and M. E. Stern. In Dry eye and the ocular surface. New York: Marcel-Dekker; 2004. 89-109 pp.

19. Tai LY, Khaw KW, Ng CM, Subrayan V. Central corneal thickness measurements with different imaging devices and ultrasound pachymetry. Cornea 2013;32:766-771.

20. Knop E, Knop N. The role of eyeassociated lymphoid tissue in corneal immune protection. J.Anat. 2005;206:271285.

21. Knop N, Knop E. Conjunctivaassociated lymphoid tissue in the human eye. Invest.Ophthalmol.Vis.Sci. 2000;41:1270-1279. 
22. Reinoso R, Martin-Sanz R, Martino M, et al. Topographical distribution and characterization of epithelial cells and intraepithelial lymphocytes in the human ocular mucosa. Mucosal Immunol. 2012;5:455-467.

23. Hamrah P, Huq SO, Gulati A, Dana R. Mechanisms of the ocular surface immune response. S. C. Pflugfelder, R. W. Beuerman and M. E. Stern. In Dry eye and the ocular surface. New York: MarcelDekker; 2004. 111-141 pp.

24. Yamagami S, Yokoo S, Usui T, Yamagami H, Amano S, Ebihara N. Distinct populations of dendritic cells in the normal human donor corneal epithelium. Invest.Ophthalmol.Vis.Sci. 2005;46:44894494.

25. Mayer WJ, Irschick UM, Moser P, et al. Characterization of antigen-presenting cells in fresh and cultured human corneas using novel dendritic cell markers. Invest.Ophthalmol.Vis. Sci. 2007;48:44594467.

26. Zhivov A, Stave J, Vollmar B, Guthoff R. In vivo confocal microscopic evaluation of langerhans cell density and distribution in the normal human corneal epithelium. Graefes Arch.Clin.Exp.Ophthalmol. 2005;243:1056-1061.

27. Mastropasqua L, Nubile M, Lanzini M, et al. Epithelial dendritic cell distribution in normal and inflamed human cornea: In vivo confocal microscopy study. Am.J.Ophthalmol. 2006;142:736-744.

28. Vantrappen L, Geboes K, Missotten L, Maudgal PC, Desmet V. Lymphocytes and langerhans cells in the normal human cornea. Invest.Ophthalmol.Vis.Sci. 1985;26:220-225.

29. Narayanan S, Redfern RL, Miller WL, Nichols KK, McDermott AM. Dry eye disease and microbial keratitis: Is there a connection? Ocul.Surf. 2013;11:75-92.

30. Mosmann TR, Sad S. The expanding universe of T-cell subsets: Th1, Th2 and more. Immunol. Today 1996;17:138-146.
31. Dardalhon V, Korn T, Kuchroo VK, Anderson AC. Role of Th1 and Th17 cells in organ-specific autoimmunity. J.Autoimmun. 2008;31:252-256.

32. Leonardi A, DeFranchis G, Zancanaro $F$, et al. Identification of local Th2 and Th0 lymphocytes in vernal conjunctivitis by cytokine flow cytometry. Invest.Ophthalmol.Vis. Sci. 1999;40:30363040.

33. Rothenberg ME, Hogan SP. The eosinophil. Annual Review of Immunology 2006;24:147-174.

34. Chen Z, O'Shea JJ. Th17 cells: A new fate for differentiating helper $T$ cells. Immunol.Res. 2008;41:87-102.

35. Korn T, Bettelli E, Oukka M, Kuchroo VK. IL-17 and Th17 cells. Annu.Rev.Immunol. 2009;27:485-517.

36. De Paiva CS, Chotikavanich S, Pangelinan SB, et al. IL-17 disrupts corneal barrier following desiccating stress. Mucosal Immunol. 2009;2:243-253.

37. Zhu J, Paul WE. CD4 T cells: Fates, functions, and faults. Blood 2008;112:15571569.

38. Dardalhon V, Awasthi A, Kwon H, et al. IL-4 inhibits TGF-beta-induced Foxp3+ T cells and, together with TGF-beta, generates IL-9+ IL-10+ Foxp3(-) effector T cells. Nat.Immunol. 2008;9:1347-1355.

39. Siemasko KF, Gao J, Calder VL, et al. In vitro expanded CD4+CD25+Foxp3+ regulatory $T$ cells maintain a normal phenotype and suppress immune-mediated ocular surface inflammation. Invest.Ophthalmol. Vis. Sci. 2008;49:54345440.

40. Yoon KC, De Paiva CS, Qi H, et al. Desiccating environmental stress exacerbates autoimmune lacrimal keratoconjunctivitis in non-obese diabetic mice. J.Autoimmun. 2008;30:212-221. 
41. De Paiva CS, Hwang CS, Pitcher JD,3rd, et al. Age-related T-cell cytokine profile parallels corneal disease severity in sjogren's syndrome-like keratoconjunctivitis sicca in CD25KO mice. Rheumatology (Oxford) 2010;49:246-258.

42. Veldhoen M, Uyttenhove C, van Snick $\mathrm{J}$, et al. Transforming growth factor-beta 'reprograms' the differentiation of T helper 2 cells and promotes an interleukin 9producing subset. Nat.Immunol. 2008;9:1341-1346.

43. Eyerich S, Eyerich K, Pennino D, et al. Th22 cells represent a distinct human $T$ cell subset involved in epidermal immunity and remodeling. J.Clin.Invest. 2009;119:35733585.

44. Jager $A$, Dardalhon $V$, Sobel RA, Bettelli E, Kuchroo VK. Th1, Th17, and Th9 effector cells induce experimental autoimmune encephalomyelitis with different pathological phenotypes. J.Immunol. 2009;183:7169-7177.

45. Soroosh P, Doherty TA. Th9 and allergic disease. Immunology 2009;127:450-458.

46. Zhang L, Li YG, Li YH, et al. Increased frequencies of Th22 cells as well as Th17 cells in the peripheral blood of patients with ankylosing spondylitis and rheumatoid arthritis. PLoS One 2012;7:e31000.

47. Mochizuki M, Sugita S, Kamoi K. Immunological homeostasis of the eye. Prog.Retin.Eye Res. 2013;33:10-27.

48. Lawrence T, Willoughby DA, Gilroy DW. Anti-inflammatory lipid mediators and insights into the resolution of inflammation. Nat.Rev.Immunol. 2002;2:787-795.

49. DEWS. The definition and classification of dry eye disease: Report of the definition and classification subcommittee of the international dry eye WorkShop. Ocul.Surf. 2007;5:75-92.

50. Miljanovic B, Dana R, Sullivan DA, Schaumberg DA. Impact of dry eye syndrome on vision-related quality of life. Am.J.Ophthalmol. 2007;143:409-415.
51. Schiffman RM, Walt JG, Jacobsen G, Doyle JJ, Lebovics G, Sumner W. Utility assessment among patients with dry eye disease. Ophthalmology 2003;110:14121419.

52. Schaumberg DA, Sullivan DA, Dana MR. Epidemiology of dry eye syndrome. Adv.Exp.Med.Biol. 2002;506:989-998.

53. McCarty CA, Bansal AK, Livingston PM, Stanislavsky YL, Taylor HR. The epidemiology of dry eye in melbourne, australia. Ophthalmology 1998;105:11141119.

54. Schaumberg DA, Sullivan DA, Buring JE, Dana MR. Prevalence of dry eye syndrome among US women. Am.J.Ophthalmol. 2003;136:318-326.

55. Moss SE, Klein R, Klein BE. Incidence of dry eye in an older population. Arch.Ophthalmol. 2004;122:369-373.

56. Schaumberg DA, Dana R, Buring JE, Sullivan DA. Prevalence of dry eye disease among US men: Estimates from the physicians' health studies. Arch.Ophthalmol. 2009;127:763-768.

57. Viso E, Rodriguez-Ares MT, Gude F. Prevalence of and associated factors for dry eye in a spanish adult population (the salnes eye study). Ophthalmic Epidemiol. 2009;16:15-21.

58. DEWS. The epidemiology of dry eye disease: Report of the definition and classification subcommittee of the international dry eye WorkShop. Ocul.Surf. 2007;5:93-107.

59. Krenzer KL, Dana MR, Ullman MD, et al. Effect of androgen deficiency on the human meibomian gland and ocular surface. J.Clin.Endocrinol.Metab. 2000;85:4874-4882.

60. Sullivan DA. Tearful relationships? sex, hormones, the lacrimal gland, and aqueous-deficient dry eye. Ocul.Surf. 2004;2:92-123.

61. Uchino $M$, Schaumberg DA, Dogru $M$, et al. Prevalence of dry eye disease among japanese visual display terminal users. Ophthalmology 2008;115:1982-1988. 
62. Battat L, Macri A, Dursun D, Pflugfelder SC. Effects of laser in situ keratomileusis on tear production, clearance, and the ocular surface. Ophthalmology 2001;108:1230-1235.

63. Altinors DD, Akca S, Akova YA, et al. Smoking associated with damage to the lipid layer of the ocular surface. Am.J.Ophthalmol. 2006;141:1016-1021.

64. Schlote T, Kadner G, Freudenthaler N. Marked reduction and distinct patterns of eye blinking in patients with moderately dry eyes during video display terminal use. Graefes Arch.Clin.Exp.Ophthalmol. 2004;242:306-312.

65. Teson M, Gonzalez-Garcia MJ, LopezMiguel $A$, et al. Influence of a controlled environment simulating an in-flight airplane cabin on dry eye disease. Invest.Ophthalmol.Vis.Sci. 2013;54:20932099.

66. Lopez-Miguel A, Teson M, MartinMontanez V, et al. Dry eye exacerbation in patients exposed to desiccating stress under controlled environmental conditions. Am.J.Ophthalmol. 2014.

67. Fox RI. Systemic diseases associated with dry eye. Int.Ophthalmol.Clin. 1994;34:71-87.

68. Baudouin C, Bourcier T, Brignole F, et al. Correlation between tear IgE levels and HLA-DR expression by conjunctival cells in allergic and nonallergic chronic conjunctivitis. Graefes Arch.Clin.Exp.Ophthalmol. 2000;238:900904.

69. Moss SE, Klein R, Klein BE. Prevalence of and risk factors for dry eye syndrome. Arch.Ophthalmol. 2000;118:1264-1268.

70. Stern ME, Schaumburg CS, Pflugfelder SC. Dry eye as a mucosal autoimmune disease. Int.Rev.Immunol. 2013;32:19-41.

71. Pflugfelder SC, Jones D, Ji Z, Afonso A, Monroy $D$. Altered cytokine balance in the tear fluid and conjunctiva of patients with sjögren's syndrome keratoconjunctivitis sicca. Curr.Eye Res. 1999;19:201-211.
72. Corrales RM, Narayanan S, Fernandez $I$, et al. Ocular mucin gene expression levels as biomarkers for the diagnosis of dry eye syndrome. Invest.Ophthalmol.Vis.Sci. 2011;52:83638369.

73. Calonge $M$, Enriquez-de-Salamanca $A$, Diebold $Y$, et al. Dry eye disease as an inflammatory disorder. Ocul.Immunol.Inflamm. 2010;18:244-253.

74. Stern ME, Siemasko KF, Gao J, Calonge M, Niederkorn JY, Pflugfelder SC. Evaluation of ocular surface inflammation in the presence of dry eye and allergic conjunctival disease. Ocul.Surf. 2005;3:S161-4.

75. Williamson J, Gibson AA, Wilson $\mathrm{T}$, Forrester JV, Whaley K, Dick WC. Histology of the lacrimal gland in keratoconjunctivitis sicca. Br.J.Ophthalmol. 1973;57:852-858.

76. Tsubota K, Fujihara T, Saito K, Takeuchi T. Conjunctival epithelium expression of HLA-DR in dry eye patients. Ophthalmologica 1999;213:16-19.

77. Kunert KS, Tisdale AS, Stern ME, Smith JA, Gipson IK. Analysis of topical cyclosporine treatment of patients with dry eye syndrome: Effect on conjunctival lymphocytes. Arch.Ophthalmol. 2000;118:1489-1496.

78. Stern ME, Gao J, Schwalb TA, et al. Conjunctival T-cell subpopulations in sjögren's and non-sjögren's patients with dry eye. Invest.Ophthalmol.Vis.Sci. 2002;43:2609-2614.

79. Smith RE. The tear film complex: Pathogenesis and emerging therapies for dry eyes. Cornea 2005;24:1-7.

80. Enriquez-de-Salamanca A, Castellanos $E$, Stern $M E$, et al. Tear cytokine and chemokine analysis and clinical correlations in evaporative-type dry eye disease. Mol.Vis. 2010;16:862-873.

81. Yoon KC, Park CS, You IC, et al. Expression of CXCL9, -10, -11, and CXCR3 in the tear film and ocular surface of patients with dry eye syndrome. Invest.Ophthalmol.Vis. Sci. 2010;51:643650. 
82. Massingale ML, Li X, Vallabhajosyula M, Chen D, Wei Y, Asbell PA. Analysis of inflammatory cytokines in the tears of dry eye patients. Cornea 2009;28:1023-1027.

83. Stern ME, Gao J, Siemasko KF, Beuerman RW, Pflugfelder SC. The role of the lacrimal functional unit in the pathophysiology of dry eye. Exp.Eye Res. 2004;78:409-416.

84. Gao J, Morgan G, Tieu D, et al. ICAM-1 expression predisposes ocular tissues to immune-based inflammation in dry eye patients and sjogrens syndrome-like MRL/Ipr mice. Exp.Eye Res. 2004;78:823835.

85. Aronni S, Cortes M, Sacchetti M, et al. Upregulation of ICAM-1 expression in the conjunctiva of patients with chronic graftversus-host disease. Eur.J.Ophthalmol. 2006;16:17-23.

86. Schrader S, Mircheff AK, Geerling G. Animal models of dry eye. Dev. Ophthalmol. 2008;41:298-312.

87. Dursun D, Wang M, Monroy D, et al. A mouse model of keratoconjunctivitis sicca. Invest.Ophthalmol.Vis.Sci. 2002;43:632638.

88. Chen Y, Chauhan SK, Lee HS, et al. Effect of desiccating environmental stress versus systemic muscarinic AChR blockade on dry eye immunopathogenesis. Invest.Ophthalmol.Vis. Sci. 2013;54:24572464.

89. Barabino S, Shen L, Chen L, Rashid S, Rolando M, Dana MR. The controlledenvironment chamber: A new mouse model of dry eye. Invest.Ophthalmol.Vis.Sci. 2005;46:2766-2771.

90. Chen W, Zhang X, Zhang J, et al. A murine model of dry eye induced by an intelligently controlled environmental system. Invest.Ophthalmol.Vis.Sci. 2008;49:1386-1391.

91. Maruyama K, Yokoi N, Takamata A, Kinoshita S. Effect of environmental conditions on tear dynamics in soft contact lens wearers. Invest.Ophthalmol.Vis.Sci. 2004;45:2563-2568.
92. Yeh S, Song X, Farley W, Li D, Stern M, Pflugfelder S. Apoptosis of ocular surface cells in experimentally induced dry eye. Invest.Ophthalmol.Vis. Sci. 2003;44:124129.

93. Pflugfelder SC, Farley W, Luo L, et al. Matrix metalloproteinase-9 knockout confers resistance to corneal epithelial barrier disruption in experimental dry eye. Am.J.Pathol. 2005;166:61-71.

94. Niederkorn JY, Stern ME, Pflugfelder $\mathrm{SC}$, et al. Desiccating stress induces T cellmediated sjogren's syndrome-like lacrimal keratoconjunctivitis. J.Immunol. 2006;176:3950-3957.

95. De Paiva CS, Villarreal AL, Corrales $\mathrm{RM}$, et al. Dry eye-induced conjunctival epithelial squamous metaplasia is modulated by interferon-gamma. Invest.Ophthalmol.Vis. Sci. 2007;48:25532560.

96. Yoon KC, De Paiva CS, Qi $\mathrm{H}$, et al. Expression of th-1 chemokines and chemokine receptors on the ocular surface of C57BL/6 mice: Effects of desiccating stress. Invest.Ophthalmol.Vis. Sci. 2007;48:2561-2569.

97. Takada K, Takiguchi M, Konno A, Inaba $M$. Autoimmunity against a tissue kallikrein in IQI/Jic mice: A model for sjogren's syndrome. J.Biol.Chem. 2005;280:39823988.

98. Stern ME, Schaumburg CS, Siemasko $\mathrm{KF}$, et al. Autoantibodies contribute to the immunopathogenesis of experimental dry eye disease. Invest.Ophthalmol.Vis.Sci. 2012;53:2062-2075.

99. Schaumburg CS, Siemasko KF, De Paiva CS, et al. Ocular surface APCs are necessary for autoreactive $\mathrm{T}$ cell-mediated experimental autoimmune lacrimal keratoconjunctivitis. J.Immunol. 2011;187:3653-3662.

100. DEWS. Management and therapy of dry eye disease: Report of the definition and classification subcommittee of the international dry eye WorkShop. Ocul.Surf. 2007;5:163-178. 
101. Marsh P, Pflugfelder SC. Topical nonpreserved methylprednisolone therapy for keratoconjunctivitis sicca in sjogren syndrome. Ophthalmology 1999;106:811816.

102. Hong S, Kim T, Chung SH, Kim EK, Seo KY. Recurrence after topical nonpreserved methylprednisolone therapy for keratoconjunctivitis sicca in sjogren's syndrome. J.Ocul.Pharmacol.Ther. 2007;23:78-82.

103. Butcher JM, Austin M, McGalliard J, Bourke RD. Bilateral cataracts and glaucoma induced by long term use of steroid eye drops. BMJ 1994;309:43.

104. Garrott HM, Walland MJ. Glaucoma from topical corticosteroids to the eyelids. Clin.Experiment.Ophthalmol. 2004;32:224226.

105. Haeck IM, Rouwen TJ, Timmer-de Mik $\mathrm{L}$, de Bruin-Weller MS, Bruijnzeel-Koomen CA. Topical corticosteroids in atopic dermatitis and the risk of glaucoma and cataracts.

J.Am.Acad.Dermatol.

2011;64:275-281.

106. Geerling G, Tauber J, Baudouin C, et al. The international workshop on meibomian gland dysfunction: Report of the subcommittee on management and treatment of meibomian gland dysfunction. Invest.Ophthalmol. Vis. Sci. 2011;52:20502064.

107. Solomon A, Rosenblatt M, Li D, et al. Doxycycline inhibition of interleukin-1 in the corneal epithelium. Am.J.Ophthalmol. 2000;130:688.

108. Dursun D, Kim MC, Solomon A, Pflugfelder SC. Treatment of recalcitrant recurrent corneal erosions with inhibitors of matrix metalloproteinase-9, doxycycline and corticosteroids. Am.J.Ophthalmol. 2001;132:8-13.

109. Salamon SM. Tetracyclines in ophthalmology. Surv.Ophthalmol.
110. Barabino S, Rolando M, Camicione $P$, et al. Systemic linoleic and gamma-linolenic acid therapy in dry eye syndrome with an inflammatory component. Cornea 2003;22:97-101.

111. Aragona $P$, Bucolo $C$, Spinella $R$, Giuffrida S, Ferreri G. Systemic omega-6 essential fatty acid treatment and pge1 tear content in sjogren's syndrome patients. Invest.Ophthalmol. Vis. Sci. 2005;46:44744479.

112. Rashid $S$, Jin $Y$, Ecoiffier $T$, Barabino S, Schaumberg DA, Dana MR. Topical omega-3 and omega-6 fatty acids for treatment of dry eye. Arch.Ophthalmol. 2008;126:219-225.

113. Turner K, Pflugfelder SC, Ji Z, Feuer WJ, Stern M, Reis BL. Interleukin-6 levels in the conjunctival epithelium of patients with dry eye disease treated with cyclosporine ophthalmic emulsion. Cornea 2000;19:492-496.

114. Yuksel B, Bozdag B, Acar M, Topaloglu E. Evaluation of the effect of topical cyclosporine $A$ with impression cytology in dry eye patients. Eur.J.Ophthalmol. 2010;20:675-679.

115. Kunert KS, Tisdale AS, Gipson IK. Goblet cell numbers and epithelial proliferation in the conjunctiva of patients with dry eye syndrome treated with cyclosporine. Arch.Ophthalmol. 2002;120:330-337.

116. Strong B, Farley $W$, Stern ME, Pflugfelder SC. Topical cyclosporine inhibits conjunctival epithelial apoptosis in experimental murine keratoconjunctivitis sicca. Cornea 2005;24:80-85.

117. Rosario N, Bielory L. Epidemiology of allergic conjunctivitis. Curr.Opin.Allergy Clin.Immunol. 2011;11:471-476.

118. Petricek I, Prost M, Popova A. The differential diagnosis of red eye: A survey of medical practitioners from eastern europe and the middle east. Ophthalmologica 2006;220:229-237. 
119. Leonardi A, De Dominicis C, Motterle L. Immunopathogenesis of ocular allergy: A schematic approach to different clinical entities. Curr.Opin.Allergy Clin.Immunol. 2007;7:429-435.

120. Leonardi $A$, Fregona IA, Plebani $M$, Secchi AG, Calder VL. Th1- and Th2-type cytokines in chronic ocular allergy. Graefes Arch.Clin.Exp.Ophthalmol. 2006;244:12401245.

121. Calonge M. Classification of ocular atopic/allergic disorders and conditions: An unsolved problem. Acta Ophthalmol.Scand.Suppl. 1999;(228):1013.

122. Wong $\mathrm{AH}$, Barg SS, Leung AK. Seasonal and perennial allergic conjunctivitis. Recent.Pat.Inflamm.Allergy Drug Discov. 2009;3:118-127.

123. Smith AF, Pitt AD, Rodruiguez AE, et al. The economic and quality of life impact of seasonal allergic conjunctivitis in a spanish setting. Ophthalmic Epidemiol. 2005;12:233-242.

124. Pitt $A D$, Smith $A F$, Lindsell $L$, Voon LW, Rose PW, Bron AJ. Economic and quality-of-life impact of seasonal allergic conjunctivitis in oxfordshire. Ophthalmic Epidemiol. 2004;11:17-33.

125. Dart JK, Buckley RJ, Monnickendan $\mathrm{M}$, Prasad J. Perennial allergic conjunctivitis: Definition, clinical characteristics and prevalence. A comparison with seasonal allergic conjunctivitis. Trans.Ophthalmol.Soc.U.K. 1986;105 ( Pt 5):513-520.

126. Anderson DF, MacLeod JD, Baddeley $\mathrm{SM}$, et al. Seasonal allergic conjunctivitis is accompanied by increased mast cell numbers in the absence of leucocyte infiltration. Clin.Exp.Allergy 1997;27:10601066.

127. Buckley RJ. Allergic eye disease--a clinical challenge. Clin.Exp.Allergy 1998;28 Suppl 6:39-43.
128. Bremond-Gignac D, Donadieu J, Leonardi $A$, et al. Prevalence of vernal keratoconjunctivitis: A rare disease? Br.J.Ophthalmol. 2008;92:1097-1102.

129. Bonini S, Coassin M, Aronni S, Lambiase A. Vernal keratoconjunctivitis. Eye (Lond) 2004;18:345-351.

130. Leonardi A. Vernal keratoconjunctivitis: Pathogenesis and treatment. Prog.Retin.Eye Res. 2002;21:319-339.

131. Leonardi A, Busca F, Motterle L, et al. Case series of 406 vernal keratoconjunctivitis patients: A demographic and epidemiological study. Acta Ophthalmol. Scand. 2006;84:406-410.

132. Abelson MB, Leonardi AA, Smith LM, Fregona IA, George MA, Secchi AG. Histaminase activity in patients with vernal keratoconjunctivitis. 1995;102:1958-1963.

Ophthalmology

133. Calonge M, Herreras JM. Clinical grading of atopic keratoconjunctivitis. Curr.Opin.Allergy Clin.Immunol. 2007;7:442-445.

134. Guglielmetti S, Dart JK, Calder V. Atopic keratoconjunctivitis and atopic dermatitis. Curr.Opin.Allergy Clin.Immunol. 2010;10:478-485.

135. Bonini S, Lambiase $A$, Matricardi $P$, Rasi G, D'Amato M, Bonini S. Atopic and vernal keratoconjunctivitis: A model for studying atopic disease. Curr.Probl.Dermatol. 1999;28:88-94.

136. Nivenius E, Montan PG, Chryssanthou $E$, Jung $K$, van Hage-Hamsten $M$, van der Ploeg I. No apparent association between periocular and ocular microcolonization and the degree of inflammation in patients with atopic keratoconjunctivitis. Clin.Exp.Allergy 2004;34:725-730.

137. Stern ME, Siemasko KF, Niederkorn JY. The Th1/Th2 paradigm in ocular allergy. Curr.Opin.Allergy Clin.Immunol. 2005;5:446-450. 
138. Yamagami S, Ebihara N, Amano SY. Chemokine receptor gene expression in giant papillae of atopic keratoconjunctivitis. Mol.Vis. 2005;11:192-200.

139. Leonardi A, Curnow SJ, Zhan $H$, Calder VL. Multiple cytokines in human tear specimens in seasonal and chronic allergic eye disease and in conjunctival fibroblast cultures. Clin.Exp.Allergy 2006;36:777-784.

140. Donshik PC, Ehlers WH, Ballow M. Giant papillary conjunctivitis. Immunol.Allergy Clin. North.Am. 2008;28:83-103, vi.

141. Forister JF, Forister EF, Yeung KK, et al. Prevalence of contact lens-related complications: UCLA contact lens study. Eye Contact Lens 2009;35:176-180.

142. Leonardi A, Borghesan F, Faggian D, Depaoli M, Secchi AG, Plebani M. Tear and serum soluble leukocyte activation markers in conjunctival allergic diseases. Am.J.Ophthalmol. 2000;129:151-158.

143. Abelson MB, Chambers WA, Smith LM. Conjunctival allergen challenge. A clinical approach to studying allergic conjunctivitis. Arch.Ophthalmol. 1990;108:84-88.

144. Irani AM, Butrus SI, Tabbara KF, Schwartz LB. Human conjunctival mast cells: Distribution of MCT and MCTC in vernal conjunctivitis and giant papillary conjunctivitis. J.Allergy Clin.Immunol. 1990;86:34-40.

145. Morgan SJ, Williams JH, Walls AF, Church MK, Holgate ST, McGill JI. Mast cell numbers and staining characteristics in the normal and allergic human conjunctiva. J.Allergy Clin.Immunol. 1991;87:111-116.

146. Jawdat DM, Albert EJ, Rowden G, Haidl ID, Marshall JS. IgE-mediated mast cell activation induces langerhans cell migration in vivo. J.Immunol. 2004;173:5275-5282.

147. Kitawaki T, Kadowaki N, Sugimoto N, et al. IgE-activated mast cells in combination with pro-inflammatory factors induce Th2-promoting dendritic cells. Int.Immunol. 2006;18:1789-1799.
148. Thurmond RL, Gelfand EW, Dunford PJ. The role of histamine $\mathrm{H} 1$ and $\mathrm{H} 4$ receptors in allergic inflammation: The search for new antihistamines. Nat.Rev.Drug Discov. 2008;7:41-53.

149. Macleod JD, Anderson DF, Baddeley SM, Holgate ST, McGill JI, Roche WR. Immunolocalization of cytokines to mast cells in normal and allergic conjunctiva. Clin.Exp.Allergy 1997;27:1328-1334.

150. Anderson DF, Zhang $S$, Bradding $P$, McGill JI, Holgate ST, Roche WR. The relative contribution of mast cell subsets to conjunctival TH2-like cytokines. Invest.Ophthalmol.Vis. Sci. 2001;42:9951001.

151. Diehl S, Rincon M. The two faces of IL-6 on Th1/Th2 differentiation. Mol.Immunol. 2002;39:531-536.

152. Kunkel EJ, Butcher EC. Chemokines and the tissue-specific migration of lymphocytes. Immunity 2002;16:1-4.

153. Hingorani M, Calder VL, Buckley RJ, Lightman SL. The role of conjunctival epithelial cells in chronic ocular allergic disease. Exp.Eye Res. 1998;67:491-500.

154. Enriquez-de-Salamanca A, Calder V, Gao J, et al. Cytokine responses by conjunctival epithelial cells: An in vitro model of ocular inflammation. Cytokine 2008;44:160-167.

155. Niederkorn JY. Immune regulatory mechanisms in allergic conjunctivitis: Insights from mouse models. Curr.Opin.Allergy Clin.Immunol. 2008;8:472-476.

156. Oboki K, Ohno T, Saito H, Nakae S. Th17 and allergy. Allergol.Int. 2008;57:121134.

157. Lambiase A, Normando EM, Vitiello L, et al. Natural killer cells in vernal keratoconjunctivitis. Mol.Vis. 2007;13:15621567.

158. Reyes NJ, Mayhew E, Chen PW, Niederkorn JY. NKT cells are necessary for maximal expression of allergic conjunctivitis. Int.Immunol. 2010;22:627636. 
159. Reyes NJ, Mayhew E, Chen PW, Niederkorn JY. Gammadelta T cells are required for maximal expression of allergic conjunctivitis. Invest.Ophthalmol.Vis.Sci. 2011;52:2211-2216.

160. Yanni JM, Sharif NA, Gamache DA, Miller ST, Weimer LK, Spellman JM. A current appreciation of sites for pharmacological intervention in allergic conjunctivitis: Effects of new topical ocular drugs. Acta Ophthalmol.Scand.Suppl. 1999;(228):33-37.

161. Cook EB, Stahl JL, Barney NP, Graziano FM. Mechanisms of antihistamines and mast cell stabilizers in ocular allergic inflammation. Curr.Drug Targets Inflamm.Allergy 2002;1:167-180.

162. Butrus S, Portela R. Ocular allergy: Diagnosis and treatment. Ophthalmol.Clin. North Am. 2005;18:485-92, v.

163. Wallace DV, Dykewicz MS, Bernstein DI, et al. The diagnosis and management of rhinitis: An updated practice parameter. J.Allergy Clin.Immunol. 2008;122:S1-84.

164. Bielory L. Ocular allergy treatment. Immunol.Allergy Clin.North.Am. 2008;28:189-224, vii.

165. Spector SL, Raizman MB. Conjunctivitis medicamentosa. J.Allergy Clin.Immunol. 1994;94:134-136.

166. Congdon NG, Schein OD, von Kulajta P, Lubomski LH, Gilbert D, Katz J. Corneal complications associated with topical ophthalmic use of nonsteroidal antiinflammatory drugs. J.Cataract Refract.Surg. 2001;27:622-631.

167. Schacke H, Docke WD, Asadullah K. Mechanisms involved in the side effects of glucocorticoids. Pharmacol. Ther. 2002;96:23-43.

168. McGhee CN, Dean S, Danesh-Meyer $\mathrm{H}$. Locally administered ocular corticosteroids: Benefits and risks. Drug Saf. 2002;25:33-55.
169. Cirillo $R$, Triggiani $M$, Siri $L$, et al. Cyclosporin A rapidly inhibits mediator release from human basophils presumably by interacting with cyclophilin. J.Immunol. 1990;144:3891-3897.

170. Stellato $C$, de Paulis A, Ciccarelli A, et al. Anti-inflammatory effect of cyclosporin A on human skin mast cells. J.Invest.Dermatol. 1992;98:800-804.

171. Cragg GM, Newman DJ, Snader KM. Natural products in drug discovery and development. J.Nat.Prod. 1997;60:52-60.

172. Samuelsson G. Drugs of natural origin: A textbook of pharmacognosy. Stockholm: Swedish Pharmaceutical Press; 1999. 551 pp.

173. Oberlies NH, Kroll DJ. Camptothecin and taxol: Historic achievements in natural products research. J.Nat.Prod. 2004;67:129-135.

174. Cragg GM, Newman DJ. Natural products: A continuing source of novel drug leads. Biochim.Biophys.Acta 2013;1830:3670-3695.

175. Douglas Kinghorn A. Pharmacognosy in the 21st century. J.Pharm.Pharmacol. 2001;53:135-148.

176. Newman DJ, Cragg GM, Snader KM. The influence of natural products upon drug discovery. Nat.Prod.Rep. 2000;17:215-234.

177. World Health Organization (WHO). Traditional medicine strategy launched. 2002;80:610.

178. Newman DJ. Natural products as leads to potential drugs: An old process or the new hope for drug discovery? J.Med.Chem. 2008;51:2589-2599.

179. Sahoo N, Choudhury K, Manchikanti $P$. Manufacturing of biodrugs: Need for harmonization in regulatory standards. BioDrugs 2009;23:217-229.

180. Rates SM. Plants as source of drugs. Toxicon 2001;39:603-613. 
181. Leiro J, Cano E, Ubeira FM, Orallo F, Sanmartin ML. In vitro effects of resveratrol on the viability and infectivity of the microsporidian encephalitozoon cuniculi. Antimicrob.Agents Chemother. 2004;48:2497-2501.

182. Spencer JP, Abd El Mohsen MM, Minihane AM, Mathers JC. Biomarkers of the intake of dietary polyphenols: Strengths, limitations and application in nutrition research. Br.J.Nutr. 2008;99:12-22.

183. Cos $P$, De Bruyne T, Apers S, Vanden Berghe D, Pieters L, Vlietinck AJ. Phytoestrogens: Recent developments. Planta Med. 2003;69:589-599.

184. Bavaresco L, Fregoni C, Cantu E, Trevisan M. Stilbene compounds: From the grapevine to wine. Drugs Exp.Clin.Res. 1999;25:57-63.

185. Rimando AM, Cuendet $M$, Desmarchelier C, Mehta RG, Pezzuto JM, Duke SO. Cancer chemopreventive and antioxidant activities of pterostilbene, a naturally occurring analogue of resveratrol. J.Agric.Food Chem. 2002;50:3453-3457.

186. Roupe KA, Remsberg CM, Yanez JA, Davies NM. Pharmacometrics of stilbenes: Seguing towards the clinic. Curr.Clin.Pharmacol. 2006;1:81-101.

187. Wang J, Matsuzaki K, Kitanaka S. Stilbene derivatives from pholidota chinensis and their anti-inflammatory activity. Chem.Pharm.Bull.(Tokyo) 2006;54:1216-1218.

188. Takaoka MJ. Of the phenolic substances of white hellebore (veratrum grandiflorum loes. fil.). J. Faculty Sci. Hokkaido Imperial University. 1940;3:1-16.

189. Berli F, D'Angelo J, Cavagnaro B, Bottini R, Wuilloud R, Silva MF. Phenolic composition in grape (vitis vinifera L. CV. malbec) ripened with different solar UV-B radiation levels by capillary zone electrophoresis. J.Agric.Food Chem. 2008;56:2892-2898.

190. Trela BC, Waterhouse AL. Resveratrol: Isomeric molar absorptivities and stability. J. Agric. Food Chem. 1996;44:1253-1257.
191. López-Hernández J, Paseiro-Losada $P$, Sanches-Silva AT, Lage-Yusty MA. Study of the changes of trans-resveratrol caused by ultraviolet light and determination of trans- and cis-resveratrol in spanish white wines. European Food Research and Technology 2007;225:789796.

192. Rius C, Abu-Taha M, Hermenegildo C, et al. Trans- but not cis-resveratrol impairs angiotensin-Il-mediated vascular inflammation through inhibition of NFkappaB activation and peroxisome proliferator-activated receptor-gamma upregulation. J.Immunol. 2010;185:37183727.

193. Merillon JM, Fauconneau B, Teguo PW, Barrier L, Vercauteren J, Huguet F. Antioxidant activity of the stilbene astringin, newly extracted from vitis vinifera cell cultures. Clin.Chem. 1997;43:1092-1093.

194. Amidon GL, Lennernas H, Shah VP, Crison JR. A theoretical basis for a biopharmaceutic drug classification: The correlation of in vitro drug product dissolution and in vivo bioavailability. Pharm.Res. 1995;12:413-420.

195. Amri A, Chaumeil JC, Sfar S, Charrueau C. Administration of resveratrol: What formulation solutions to bioavailability limitations? J.Control.Release 2012;158:182-193.

196. Goldberg DM, Yan J, Soleas GJ. Absorption of three wine-related polyphenols in three different matrices by healthy subjects. Clin.Biochem. 2003;36:79-87.

197. Boocock DJ, Faust GE, Patel KR, et al. Phase I dose escalation pharmacokinetic study in healthy volunteers of resveratrol, a potential cancer chemopreventive agent. Cancer Epidemiol.Biomarkers Prev. 2007;16:12461252.

198. Renaud S, de Lorgeril M. Wine, alcohol, platelets, and the french paradox for coronary heart disease. Lancet 1992;339:1523-1526. 
199. Jang M, Cai L, Udeani GO, et al. Cancer chemopreventive activity of resveratrol, a natural product derived from grapes. Science 1997;275:218-220.

200. Fremont L. Biological effects of resveratrol. Life Sci. 2000;66:663-673.

201. Hung LM, Chen JK, Huang SS, Lee RS, Su MJ. Cardioprotective effect of resveratrol, a natural antioxidant derived from grapes. Cardiovasc. Res. 2000;47:549555.

202. Baur JA, Sinclair DA. Therapeutic potential of resveratrol: The in vivo evidence. Nat.Rev.Drug Discov. 2006;5:493-506.

203. de la Lastra CA, Villegas I. Resveratrol as an antioxidant and pro-oxidant agent: Mechanisms and clinical implications. Biochem.Soc.Trans. 2007;35:1156-1160.

204. Leonard SS, Xia C, Jiang BH, et al. Resveratrol scavenges reactive oxygen species and effects radical-induced cellular responses.

Biochem.Biophys.Res.Commun.

2003;309:1017-1026.

205. Rotondo S, Rajtar G, Manarini S, et al. Effect of trans-resveratrol, a natural polyphenolic compound, on human polymorphonuclear leukocyte function. Br.J.Pharmacol. 1998;123:1691-1699.

206. Yen GC, Duh PD, Lin CW. Effects of resveratrol and 4-hexylresorcinol on hydrogen peroxide-induced oxidative DNA damage in human lymphocytes. Free Radic.Res. 2003;37:509-514.

207. Cerqueira AM, Khaper N, Lees SJ, Ulanova $M$. The antioxidant resveratrol down-regulates inflammation in an in-vitro model of pseudomonas aeruginosa infection of lung epithelial cells. Can.J.Physiol.Pharmacol. $\quad$ 2013;91:248255.
208. Kode A, Rajendrasozhan S, Caito S, Yang SR, Megson IL, Rahman I. Resveratrol induces glutathione synthesis by activation of Nrf2 and protects against cigarette smoke-mediated oxidative stress in human lung epithelial cells. Am.J.Physiol.Lung Cell.Mol.Physiol. 2008;294:L478-88.

209. Blackwell TS, Christman JW. The role of nuclear factor-kappa B in cytokine gene regulation. Am.J.Respir.Cell Mol.Biol. 1997;17:3-9.

210. Newton R, Kuitert LM, Bergmann M, Adcock IM, Barnes PJ. Evidence for involvement of NF-kappaB in the transcriptional control of COX-2 gene expression by IL-1beta. Biochem.Biophys.Res. Commun. 1997;237:28-32.

211. Manna SK, Mukhopadhyay A, Aggarwal BB. Resveratrol suppresses TNFinduced activation of nuclear transcription factors NF-kappa B, activator protein-1, and apoptosis: Potential role of reactive oxygen intermediates and lipid peroxidation. J.Immunol. 2000;164:6509-6519.

212. Kundu JK, Shin YK, Kim SH, Surh YJ. Resveratrol inhibits phorbol ester-induced expression of COX-2 and activation of NFkappaB in mouse skin by blocking IkappaB kinase activity. Carcinogenesis 2006;27:1465-1474.

213. Kundu JK, Shin YK, Surh YJ. Resveratrol modulates phorbol esterinduced pro-inflammatory signal transduction pathways in mouse skin in vivo: NF-kappaB and $A P-1$ as prime targets.

Biochem.Pharmacol.

2006;72:1506-1515.

214. Sharma S, Chopra K, Kulkarni SK, Agrewala JN. Resveratrol and curcumin suppress immune response through CD28/CTLA-4 and CD80 co-stimulatory pathway. Clin.Exp.Immunol. 2007;147:155163.

215. Svajger $U$, Obermajer $N$, Jeras $M$. Dendritic cells treated with resveratrol during differentiation from monocytes gain substantial tolerogenic properties upon activation. Immunology 2010;129:525-535. 
216. Buttari B, Profumo E, Facchiano F, et al. Resveratrol prevents dendritic cell maturation in response to advanced glycation end products. Oxid Med.Cell.Longev 2013;2013:574029.

217. Wung BS, Hsu MC, Wu CC, Hsieh CW. Resveratrol suppresses IL-6-induced ICAM-1 gene expression in endothelial cells: Effects on the inhibition of STAT3 phosphorylation. Life Sci. 2005;78:389-397.

218. Park HJ, Jeong SK, Kim SR, et al. Resveratrol inhibits porphyromonas gingivalis lipopolysaccharide-induced endothelial adhesion molecule expression by suppressing NF-kappaB activation. Arch.Pharm.Res. 2009;32:583-591.

219. Zaidi SF, Ahmed K, Yamamoto T, et al. Effect of resveratrol on helicobacter pylori-induced interleukin-8 secretion, reactive oxygen species generation and morphological changes in human gastric epithelial cells. Biol.Pharm.Bull. 2009;32:1931-1935.

220. Houser KR, Johnson DK, Ishmael FT. Anti-inflammatory effects of methoxyphenolic compounds on human airway cells. J.Inflamm.(Lond) 2012;9:69255-9-6.

221. Donnelly LE, Newton R, Kennedy GE, et al. Anti-inflammatory effects of resveratrol in lung epithelial cells: Molecular mechanisms. Am.J.Physiol.Lung Cell.Mol.Physiol. 2004;287:L774-83.

222. Lee M, Kim S, Kwon OK, Oh SR, Lee HK, Ahn K. Anti-inflammatory and antiasthmatic effects of resveratrol, a polyphenolic stilbene, in a mouse model of allergic asthma. Int.Immunopharmacol. 2009;9:418-424.

223. Okada Y, Oh-oka K, Nakamura Y, et al. Dietary resveratrol prevents the development of food allergy in mice. PLOS One 2012;7:e44338.

224. Kubota S, Kurihara T, Mochimaru H, et al. Prevention of ocular inflammation in endotoxin-induced uveitis with resveratrol by inhibiting oxidative damage and nuclear factor-kappaB activation. Invest.Ophthalmol.Vis.Sci. 2009;50:35123519.
225. Fuentes ME, Durham SK, Swerdel $M R$, et al. Controlled recruitment of monocytes and macrophages to specific organs through transgenic expression of monocyte chemoattractant protein-1. J.Immunol. 1995;155:5769-5776.

226. Becker MD, Garman K, Whitcup SM, Planck SR, Rosenbaum JT. Inhibition of leukocyte sticking and infiltration, but not rolling, by antibodies to ICAM-1 and LFA-1 in murine endotoxin-induced uveitis. Invest.Ophthalmol.Vis. Sci. 2001;42:25632566.

227. Doganay S, Borazan $M$, Iraz $M$, Cigremis $Y$. The effect of resveratrol in experimental cataract model formed by sodium selenite. Curr.Eye Res. 2006;31:147-153.

228. Li G, Luna C, Navarro ID, et al. Resveratrol prevention of oxidative stress damage to lens epithelial cell cultures is mediated by forkhead box $\mathrm{O}$ activity. Invest.Ophthalmol. Vis. Sci. 2011;52:43954401.

229. Zheng Y, Liu Y, Ge J, et al. Resveratrol protects human lens epithelial cells against $\mathrm{H} 2 \mathrm{O} 2$-induced oxidative stress by increasing catalase, SOD-1, and HO-1 expression. Mol.Vis. 2010;16:1467-1474.

230. Luna C, Li G, Liton PB, et al. Resveratrol prevents the expression of glaucoma markers induced by chronic oxidative stress in trabecular meshwork cells. Food Chem.Toxicol. 2009;47:198204.

231. Marino A, Santoro G, Spataro F, et al. Resveratrol role in staphylococcus aureusinduced corneal inflammation. Pathog.Dis. 2013;68:61-64.

232. Kennedy M, Kim KH, Harten B, et al. Ultraviolet irradiation induces the production of multiple cytokines by human corneal cells. 1997;38:2483-2491.

233. Viiri J, Jauhonen HM, Kauppinen A, et al. Cis-urocanic acid suppresses UV-Binduced interleukin- 6 and -8 secretion and cytotoxicity in human corneal and conjunctival epithelial cells in vitro. Mol.Vis. 2009;15:1799-1805. 
234. Black AT, Gordon MK, Heck DE, Gallo MA, Laskin DL, Laskin JD. UVB light regulates expression of antioxidants and inflammatory mediators in human corneal epithelial cells. Biochem.Pharmacol. 2011;81:873-880.

235. Chou WW, Chen KC, Wang YS, Wang JY, Liang CL, Juo SH. The role of SIRT1/AKT/ERK pathway in ultraviolet $B$ induced damage on human retinal pigment epithelial cells. Toxicol.in.Vitro. 2013;27:1728-1736.

236. Kubota S, Kurihara T, Ebinuma M, et al. Resveratrol prevents light-induced retinal degeneration via suppressing activator protein-1 activation. Am.J.Pathol. 2010;177:1725-1731.

237. Alvesalo J, Vuorela $\mathrm{H}$, Tammela $\mathrm{P}$, Leinonen M, Saikku P, Vuorela P. Inhibitory effect of dietary phenolic compounds on chlamydia pneumoniae in cell cultures. Biochem.Pharmacol. 2006;71:735-741.

238. Song JM, Lee KH, Seong BL. Antiviral effect of catechins in green tea on influenza virus. Antiviral Res. 2005;68:66-74.

239. Romagnolo DF, Selmin OI. Flavonoids and cancer prevention: A review of the evidence. J.Nutr.Gerontol.Geriatr. 2012;31:206-238.

240. Gonzalez R, Ballester I, LopezPosadas R, et al. Effects of flavonoids and other polyphenols on inflammation. Crit.Rev.Food Sci.Nutr. 2011;51:331-362.

241. Sampson L, Rimm E, Hollman PC, de Vries $\mathrm{JH}$, Katan MB. Flavonol and flavone intakes in US health professionals. J.Am.Diet.Assoc. 2002;102:1414-1420.

242. Harwood M, Danielewska-Nikiel B, Borzelleca JF, Flamm GW, Williams GM, Lines TC. A critical review of the data related to the safety of quercetin and lack of evidence of in vivo toxicity, including lack of genotoxic/carcinogenic properties. Food Chem. Toxicol. 2007;45:2179-2205.

243. Egert S, Wolffram S, Bosy-Westphal $A$, et al. Daily quercetin supplementation dose-dependently increases plasma quercetin concentrations in healthy humans. J.Nutr. 2008;138:1615-1621.
244. Okamoto T. Safety of quercetin for clinical application (review). Int.J.Mol.Med. 2005;16:275-278.

245. Davis JM, Murphy EA, Carmichael MD. Effects of the dietary flavonoid quercetin upon performance and health. Curr.Sports Med.Rep. 2009;8:206-213.

246. Kelly GS. Quercetin. monograph. Altern.Med.Rev. 2011;16:172-194.

247. Rice-Evans CA, Miller NJ, Paganga G. Structure-antioxidant activity relationships of flavonoids and phenolic acids. Free Radic.Biol.Med. 1996;20:933-956.

248. Kamaraj S, Vinodhkumar R, Anandakumar $P$, Jagan $S$, Ramakrishnan $G$, Devaki T. The effects of quercetin on antioxidant status and tumor markers in the lung and serum of mice treated with benzo(a)pyrene. Biol.Pharm.Bull. 2007;30:2268-2273.

249. Egert S, Bosy-Westphal A, Seiberl J, et al. Quercetin reduces systolic blood pressure and plasma oxidised low-density lipoprotein concentrations in overweight subjects with a high-cardiovascular disease risk phenotype: A double-blinded, placebocontrolled cross-over study. Br.J.Nutr. 2009;102:1065-1074.

250. Egert S, Boesch-Saadatmandi C, Wolffram S, Rimbach G, Muller MJ. Serum lipid and blood pressure responses to quercetin vary in overweight patients by apolipoprotein E genotype. J.Nutr. 2010;140:278-284.

251. Scholten SD, Sergeev IN. Long-term quercetin supplementation reduces lipid peroxidation but does not improve performance in endurance runners. Open Access J.Sports Med. 2013;4:53-61.

252. Hamalainen $M$, Nieminen $R$, Vuorela $P$, Heinonen $M$, Moilanen $E$. Antiinflammatory effects of flavonoids: Genistein, kaempferol, quercetin, and daidzein inhibit STAT-1 and NF-kappaB activations, whereas flavone, isorhamnetin, naringenin, and pelargonidin inhibit only NF-kappaB activation along with their inhibitory effect on iNOS expression and NO production in activated macrophages. Mediators Inflamm. 2007;2007:45673. 
253. Lee KW, Kang NJ, Heo YS, et al. Raf and MEK protein kinases are direct molecular targets for the chemopreventive effect of quercetin, a major flavonol in red wine. Cancer Res. 2008;68:946-955.

254. Comalada M, Camuesco D, Sierra S, et al. In vivo quercitrin anti-inflammatory effect involves release of quercetin, which inhibits inflammation through downregulation of the NF-kappaB pathway. Eur.J.Immunol. 2005;35:584-592.

255. Manjeet KR, Ghosh B. Quercetin inhibits LPS-induced nitric oxide and tumor necrosis factor-alpha production in murine macrophages. Int.J.Immunopharmacol. 1999;21:435-443.

256. Xagorari A, Papapetropoulos A, Mauromatis $A$, Economou $M$, Fotsis $T$, Roussos C. Luteolin inhibits an endotoxinstimulated phosphorylation cascade and proinflammatory cytokine production in macrophages. J.Pharmacol.Exp.Ther. 2001;296:181-187.

257. Geraets L, Moonen HJ, Brauers K, Wouters EF, Bast A, Hageman GJ. Dietary flavones and flavonoles are inhibitors of poly(ADP-ribose)polymerase-1 in pulmonary epithelial cells. J.Nutr. 2007;137:2190-2195.

258. Bureau G, Longpre F, Martinoli MG. Resveratrol and quercetin, two natural polyphenols, reduce apoptotic neuronal cell death induced by neuroinflammation. J.Neurosci.Res. 2008;86:403-410.

259. Qureshi AA, Tan X, Reis JC, et al. Inhibition of nitric oxide in LPS-stimulated macrophages of young and senescent mice by delta-tocotrienol and quercetin. Lipids Health.Dis. 2011;10:239-511X-10-239.

260. Natarajan K, Manna SK, Chaturvedi MM, Aggarwal BB. Protein tyrosine kinase inhibitors block tumor necrosis factorinduced activation of nuclear factor-kappaB, degradation of IkappaBalpha, nuclear translocation of p65, and subsequent gene expression. Arch.Biochem.Biophys. 1998;352:59-70
261. Cho SY, Park SJ, Kwon MJ, et al. Quercetin suppresses proinflammatory cytokines production through MAP kinases andNF-kappaB pathway in lipopolysaccharide-stimulated macrophage. Mol.Cell.Biochem. 2003;243:153-160.

262. Garcia-Mediavilla V, Crespo I, Collado PS, et al. The anti-inflammatory flavones quercetin and kaempferol cause inhibition of inducible nitric oxide synthase, cyclooxygenase-2 and reactive C-protein, and down-regulation of the nuclear factor kappaB pathway in chang liver cells. Eur.J.Pharmacol. 2007;557:221-229.

263. Nanua S, Zick SM, Andrade JE, et al. Quercetin blocks airway epithelial cell chemokine expression. Am.J.Respir.Cell Mol.Biol. 2006;35:602-610.

264. Wadsworth TL, McDonald TL, Koop DR. Effects of ginkgo biloba extract (EGb 761) and quercetin on lipopolysaccharideinduced signaling pathways involved in the release of tumor necrosis factor-alpha. Biochem.Pharmacol. 2001;62:963-974.

265. Muthian G, Bright JJ. Quercetin, a flavonoid phytoestrogen, ameliorates experimental allergic encephalomyelitis by blocking IL-12 signaling through JAK-STAT pathway in T lymphocyte. J.Clin.Immunol. 2004;24:542-552.

266. Milenkovic M, Arsenovic-Ranin N, Stojic-Vukanic Z, Bufan B, Vucicevic D, Jancic I. Quercetin ameliorates experimental autoimmune myocarditis in rats. J.Pharm.Pharm.Sci. 2010;13:311-319.

267. Joskova M, Franova S, Sadlonova V. Acute bronchodilator effect of quercetin in experimental allergic asthma. Bratisl.Lek.Listy 2011;112:9-12.

268. Jung $\mathrm{CH}$, Lee JY, Cho $\mathrm{CH}$, Kim CJ. Anti-asthmatic action of quercetin and rutin in conscious guinea-pigs challenged with aerosolized ovalbumin. Arch.Pharm.Res. 2007;30:1599-1607.

269. Moon $\mathrm{H}$, Choi HH, Lee JY, Moon HJ, Sim SS, Kim CJ. Quercetin inhalation inhibits the asthmatic responses by exposure to aerosolized-ovalbumin in conscious guinea-pigs. Arch.Pharm.Res. 2008;31:771-778. 
270. Rogerio AP, Dora CL, Andrade EL, et al. Anti-inflammatory effect of quercetinloaded microemulsion in the airways allergic inflammatory model in mice. Pharmacol.Res. 2010;61:288-297.

271. Rogerio AP, Kanashiro A, Fontanari C, et al. Anti-inflammatory activity of quercetin and isoquercitrin in experimental murine allergic asthma. Inflamm.Res. 2007;56:402408.

272. Park HJ, Lee CM, Jung ID, et al. Quercetin regulates Th1/Th2 balance in a murine model of asthma. Int.Immunopharmacol. 2009;9:261-267.

273. Shishehbor F, Behroo L, Ghafouriyan Broujerdnia M, Namjoyan F, Latifi SM. Quercetin effectively quells peanut-induced anaphylactic reactions in the peanut sensitized rats. Iran.J.Allergy Asthma Immunol. 2010;9:27-34.

274. Varma SD, Mikuni I, Kinoshita JH. Flavonoids as inhibitors of lens aldose reductase. Science 1975;188:1215-1216.

275. Cornish KM, Williamson G, Sanderson $\mathrm{J}$. Quercetin metabolism in the lens: Role in inhibition of hydrogen peroxide induced cataract. Free Radic.Biol.Med. 2002;33:6370.

276. Cao XG, Li XX, Bao YZ, Xing NZ, Chen $Y$. Responses of human lens epithelial cells to quercetin and DMSO. Invest.Ophthalmol.Vis.Sci. 2007;48:37143718.

277. Romero J, Marak GE,Jr, Rao NA. Pharmacologic modulation of acute ocular inflammation with quercetin. Ophthalmic Res. 1989;21:112-117.

278. Kaidzu S, Tanito M, Kim Y-, Koyama Y, Ohira A. Quercetin protect the rat retina from light-induced damage. Invest.Ophthalmol.Vis.Sci. 2005;46:1623.

279. Jiang Q, Cao C, Zhou C, et al. Quercetin attenuates UV- and $\mathrm{H}(2) \mathrm{O}(2)$ induced decrease of collagen type $I$ in cultured human lens epithelial cells. J.Ocul.Pharmacol.Ther. 2008;24:164-174.
280. Pauloin T, Dutot M, Joly F, Warnet JM, Rat P. High molecular weight hyaluronan decreases UVB-induced apoptosis and inflammation in human epithelial corneal cells. Mol.Vis. 2009;15:577-583.

281. Diebold $Y$, Calonge $M$, Enriquez de Salamanca $A$, et al. Characterization of a spontaneously immortalized cell line (IOBA$\mathrm{NHC})$ from normal human conjunctiva. Invest. Ophthalmol.Vis.Sci. 2003;44:42634274.

282. Araki-Sasaki K, Ohashi $Y$, Sasabe $T$, et al. An SV40-immortalized human corneal epithelial cell line and its characterization. Invest.Ophthalmol.Vis.Sci. $\quad 1995 ; 36: 614-$ 621.

283. O'Brien J, Wilson I, Orton T, Pognan F. Investigation of the alamar blue (resazurin) fluorescent dye for the assessment of mammalian cell cytotoxicity. Eur.J.Biochem. 2000;267:5421-5426.

284. Smith PK, Krohn RI, Hermanson GT, et al. Measurement of protein using bicinchoninic acid. Anal.Biochem. 1985;150:76-85.

285. Vignali DA. Multiplexed particle-based flow cytometric assays. J.Immunol.Methods 2000;243:243-255.

286. Morgan E, Varro R, Sepulveda $\mathrm{H}$, et al. Cytometric bead array: A multiplexed assay platform with applications in various areas of biology. Clin.Immunol. 2004;110:252-266.

287. Laemmli UK. Cleavage of structural proteins during the assembly of the head of bacteriophage T4. Nature 1970;227:680685.

288. Jameel A, Ooi KG, Jeffs NR, Galatowicz G, Lightman SL, Calder VL. Statin modulation of human T-cell proliferation, IL-1beta and IL-17 production, and IFN-gamma T cell expression: Synergy with conventional immunosuppressive agents. Int.J.Inflam 2013;2013:434586.

289. Lemp MA. Report of the national eye Institute/Industry workshop on clinical trials in dry eyes. CLAO J. 1995;21:221-232. 
290. De Paiva CS, Villarreal AL, Corrales $\mathrm{RM}$, et al. Dry eye-induced conjunctival epithelial squamous metaplasia is modulated by interferon-gamma. Invest.Ophthalmol.Vis.Sci. 2007;48:25532560.

291. Schaumburg CS, Siemasko KF, De Paiva CS, et al. Ocular surface APCs are necessary for autoreactive $\mathrm{T}$ cell-mediated experimental autoimmune lacrimal keratoconjunctivitis. J.Immunol. 2011;187:3653-3662.

292. Calder VL, Galatowicz G. Human cord blood mast cell IgE cross-linking: An in vitro model of conjunctival mast cell responses? Invest.Ophthalmol.Vis. Sci. 2005;46:934.

293. Galatowicz G, Ajayi Y, Stern ME, Calder VL. Ocular anti-allergic compounds selectively inhibit human mast cell cytokines in vitro and conjunctival cell infiltration in vivo. Clin.Exp.Allergy 2007;37:1648-1656.

294. Halliwell B. Are polyphenols antioxidants or pro-oxidants? what do we learn from cell culture and in vivo studies? Arch.Biochem.Biophys. 2008;476:107-112.

295. Ketsawatsakul U. Modulation by bicarbonate the protective effects of phenolic antioxidants on peroxynitritemediated cell cytotoxicity. ScienceAsia 2007;33:273-282.

296. Yang NC, Lee CH, Song TY. Evaluation of resveratrol oxidation in vitro and the crucial role of bicarbonate ions. Biosci.Biotechnol.Biochem. 2010;74:63-68.

297. Dugas TR, Morel DW, Harrison EH. Novel cell culture medium for use in oxidation experiments provides insights into mechanisms of endothelial cell-mediated oxidation of LDL. In Vitro Cell.Dev.Biol.Anim. 2000;36:571-577.

298. Lewinska A, Wnuk M, Slota E, Bartosz G. Total anti-oxidant capacity of cell culture media. 2007;34:781-786
299. Babich $H$, Liebling EJ, Burger RF, Zuckerbraun HL, Schuck AG. Choice of DMEM, formulated with or without pyruvate, plays an important role in assessing the in vitro cytotoxicity of oxidants and prooxidant nutraceuticals. In Vitro Cell.Dev.Biol.Anim. 2009;45:226-233.

300. Long LH, Halliwell B. Artefacts in cell culture: Pyruvate as a scavenger of hydrogen peroxide generated by ascorbate or epigallocatechin gallate in cell culture media. Biochem.Biophys.Res.Commun. 2009;388:700-704.

301. Stoddard AR, Koetje LR, Mitchell AK, Schotanus MP, Ubels JL. Bioavailability of antioxidants applied to stratified human corneal epithelial cells. J.Ocul.Pharmacol.Ther. 2013;29:681-687.

302. Chen M, Hu DN, Pan Z, Lu CW, Xue $\mathrm{CY}$, Aass I. Curcumin protects against hyperosmoticity-induced IL-1beta elevation in human corneal epithelial cell via MAPK pathways. Exp.Eye Res. 2010;90:437-443.

303. Cavet ME, Harrington KL, Vollmer TR, Ward KW, Zhang JZ. Anti-inflammatory and anti-oxidative effects of the green tea polyphenol epigallocatechin gallate in human corneal epithelial cells. Mol.Vis. 2011;17:533-542.

304. Berra A, Berra M. Hyperosmolarity induce nuclear translocation of NF-kB in human conjunctival epithelial cells. Invest.Ophthalmol. Vis. Sci. 2005;46:4402.

305. Li D, Luo L, Chen Z, Kim H, Song XJ, Pflugfelder SC. JNK and ERK MAP kinases mediate induction of IL-1 $\beta$, TNF- $\alpha$ and IL-8 following hyperosmolar stress in human limbal epithelial cells. Exp.Eye Res. 2006;82:588-596.

306. Kumari S, Bhol KC, Rehman F, Foster CS, Ahmed AR. Interleukin 1 components in cicatricial pemphigoid. role in intravenous immunoglobulin therapy. Cytokine 2001;14:218-224.

307. Caproni M, Torchia D, Schincaglia E, et al. Expression of cytokines and chemokine receptors in the cutaneous lesions of erythema multiforme and stevens-johnson syndrome/toxic epidermal necrolysis. Br.J.Dermatol. 2006;155:722728. 
308. Cook EB, Stahl JL, Graziano FM, Barney NP. Regulation of the receptor for TNFalpha, TNFR1, in human conjunctival epithelial cells. Invest.Ophthalmol.Vis.Sci. 2008;49:3992-3998.

309. Leonardi $A$, Brun $P$, Tavolato $M$, Plebani M, Abatangelo G, Secchi AG. Tumor necrosis factor-alpha (TNF-alpha) in seasonal allergic conjunctivitis and vernal keratoconjunctivitis. Eur.J.Ophthalmol. 2003;13:606-610.

310. Leonardi A. Allergy and allergic mediators in tears. Exp.Eye Res. 2013;117:106-117.

311. Leonardi A, Borghesan F, DePaoli M, Plebani M, Secchi AG. Procollagens and inflammatory cytokine concentrations in tarsal and limbal vernal keratoconjunctivitis. Exp.Eye Res. 1998;67:105-112.

312. Wakamatsu TH, Dogru M, Ayako I, et al. Evaluation of lipid oxidative stress status and inflammation in atopic ocular surface disease. Mol.Vis. 2010;16:2465-2475.

313. Kishimoto T. Interleukin-6: Discovery of a pleiotropic cytokine. Arthritis Res. Ther. 2006;8 Suppl 2:S2.

314. Hirano T, Akira S, Taga T, Kishimoto $\mathrm{T}$. Biological and clinical aspects of interleukin 6. Immunol.Today 1990;11:443449.

315. Kishimoto T. IL-6: From its discovery to clinical applications. Int.Immunol. 2010;22:347-352.

316. Tishler M, Yaron I, Geyer O, Shirazi I, Naftaliev E, Yaron M. Elevated tear interleukin-6 levels in patients with sjögren syndrome. Ophthalmology 1998;105:23272329.

317. Lam H, Bleiden L, de Paiva CS, Farley W, Stern ME, Pflugfelder SC. Tear cytokine profiles in dysfunctional tear syndrome. Am.J.Ophthalmol. 2009;147:198-205. e1.

318. Yoon KC, Jeong IY, Park YG, Yang SY. Interleukin-6 and tumor necrosis factoralpha levels in tears of patients with dry eye syndrome. Cornea 2007;26:431-437.
319. Baggiolini $M$, Dewald $B$, Moser $B$. Human chemokines: An update. Annu.Rev.Immunol. 1997;15:675-705.

320. Ghasemi H, Ghazanfari T, Yaraee R, Faghihzadeh S, Hassan ZM. Roles of IL-8 in ocular inflammations: A review. Ocul.Immunol.Inflamm. 2011;19:401-412.

321. Erdogan-Poyraz C, Mocan MC, Bozkurt B, Gariboglu S, Irkec M, Orhan M. Elevated tear interleukin-6 and interleukin-8 levels in patients with conjunctivochalasis. Cornea 2009;28:189-193.

322. Miyoshi T, Fukagawa K, Shimmura S, et al. Interleukin-8 concentrations in conjunctival epithelium brush cytology samples correlate with neutrophil, eosinophil infiltration, and corneal damage. Cornea 2001;20:743-747.

323. Luster AD, Ravetch JV. Biochemical characterization of a gamma interferoninducible cytokine (IP-10). J.Exp.Med. 1987;166:1084-1097.

324. Taub DD, Lloyd AR, Conlon K, et al. Recombinant human interferon-inducible protein 10 is a chemoattractant for human monocytes and $\mathrm{T}$ lymphocytes and promotes $\mathrm{T}$ cell adhesion to endothelial cells. J.Exp.Med. 1993;177:1809-1814.

325. Taub DD, Sayers TJ, Carter CR, Ortaldo JR. Alpha and beta chemokines induce NK cell migration and enhance NKmediated cytolysis. J.Immunol. 1995;155:3877-3888.

326. Qin S, Rottman JB, Myers $P$, et al. The chemokine receptors CXCR3 and CCR5 mark subsets of $T$ cells associated with certain inflammatory reactions. J.Clin.Invest. 1998;101:746-754.

327. Kim I, Ryan AM, Rohan R, et al. Constitutive expression of VEGF, VEGFR1 , and VEGFR-2 in normal eyes. Invest.Ophthalmol.Vis. Sci. 1999;40:21152121.

328. Jin J, Guan M, Sima J, et al. Decreased pigment epithelium-derived factor and increased vascular endothelial growth factor levels in pterygia. Cornea 2003;22:473-477. 
329. Aiello LP, Avery RL, Arrigg PG, et al. Vascular endothelial growth factor in ocular fluid of patients with diabetic retinopathy and other retinal disorders. N.Engl.J.Med. 1994;331:1480-1487.

330. Philipp W, Speicher L, Humpel C. Expression of vascular endothelial growth factor and its receptors in inflamed and vascularized human corneas. Invest.Ophthalmol.Vis.Sci. 2000;41:25142522.

331. Bianchi E, Scarinci F, Grande C, et al. Immunohistochemical profile of VEGF, TGF-beta and PGE(2) in human pterygium and normal conjunctiva: Experimental study and review of the literature. Int.J.Immunopathol.Pharmacol. 2012;25:607-615.

332. Leonardi A, Sathe S, Bortolotti $M$, Beaton A, Sack R. Cytokines, matrix metalloproteases, angiogenic and growth factors in tears of normal subjects and vernal keratoconjunctivitis patients. Allergy 2009;64:710-717.

333. Abu El-Asrar AM, Al-Mansouri S, Tabbara KF, Missotten L, Geboes K. Immunopathogenesis of conjunctival remodelling in vernal keratoconjunctivitis. Eye (Lond) 2006;20:71-79.

334. Losso JN, Truax RE, Richard G. Trans-resveratrol inhibits hyperglycemiainduced inflammation and connexin downregulation in retinal pigment epithelial cells. J.Agric.Food Chem. 2010;58:82468252.

335. Chen R, Hollborn M, Grosche A, et al. Effects of the vegetable polyphenols epigallocatechin-3-gallate, luteolin, apigenin, myricetin, quercetin, and cyanidin in primary cultures of human retinal pigment epithelial cells. Mol.Vis. 2014;20:242-258.

336. Koh CH, Lee HS, Chung SK. Effect of topical epigallocatechin gallate on corneal neovascularization in rabbits. Cornea 2014;33:527-532.

337. Singh N, Amin S, Richter E, et al. Flt-1 intraceptors inhibit hypoxia-induced VEGF expression in vitro and corneal neovascularization in vivo. Invest.Ophthalmol.Vis. Sci. 2005;46:16471652.
338. Ricciotti E, FitzGerald GA. Prostaglandins and inflammation. Arterioscler.Thromb. Vasc. Biol. 2011;31:986-1000.

339. Smith WL, DeWitt DL, Garavito RM. Cyclooxygenases: Structural, cellular, and molecular biology. Annu.Rev.Biochem. 2000;69:145-182.

340. Dubois RN, Abramson SB, Crofford L, et al. Cyclooxygenase in biology and disease. FASEB J. 1998;12:1063-1073.

341. Chiang CC, Cheng YW, Lin CL, et al. Cyclooxygenase 2 expression in pterygium. Mol.Vis. 2007;13:635-638.

342. Shim J, Park C, Lee HS, et al. Change in prostaglandin expression levels and synthesizing activities in dry eye disease. Ophthalmology 2012;119:2211-2219.

343. Schalnus R. Topical nonsteroidal antiinflammatory therapy in ophthalmology. Ophthalmologica 2003;217:89-98.

344. Zigman S. Environmental near-UV radiation and cataracts. Optometry Vision Sci. 1995;72:899-901.

345. Blumthaler M, Ambach W, Daxecker $F$. On the threshold radiant exposure for keratitis solaris. Invest.Ophthalmol.Vis. Sci. 1987;28:1713-1716.

346. Ringvold A. Cornea and ultraviolet radiation. Acta Ophthalmol.(Copenh) 1980;58:63-68.

347. Taylor H. The biological effects of UV$B$ on the eye. Photochem.Photobiol. 1989;50:489-492.

348. Coroneo MT. Pterygium as an early indicator of ultraviolet insolation: A hypothesis. Br.J.Ophthalmol. 1993;77:734739.

349. Di Girolamo N, Kumar RK, Coroneo MT, Wakefield D. UVB-mediated induction of interleukin- 6 and -8 in pterygia and cultured human pterygium epithelial cells. Invest. Ophthalmol. Vis.Sci. 2002;43:34303437. 
350. Taylor HR, West SK, Rosenthal FS, Munoz B, Newland HS, Emmett EA. Corneal changes associated with chronic UV irradiation. Arch.Ophthalmol. 1989;107:1481-1484.

351. Kau HC, Tsai CC, Lee CF, et al. Increased oxidative DNA damage, 8hydroxydeoxy- guanosine, in human pterygium. Eye (Lond) 2006;20:826-831.

352. Cejkova J, Stipek S, Crkovska J, et al. UV rays, the prooxidant/antioxidant imbalance in the cornea and oxidative eye damage. Physiol.Res. 2004;53:1-10.

353. Cejkova J, Ardan T, Cejka C, Kovaceva J, Zidek Z. Irradiation of the rabbit cornea with UVB rays stimulates the expression of nitric oxide synthasesgenerated nitric oxide and the formation of cytotoxic nitrogen-related oxidants. Histol.Histopathol. 2005;20:467-473.

354. Cejkova J, Stipek S, Crkovska J, Ardan T. Changes of superoxide dismutase, catalase and glutathione peroxidase in the corneal epithelium after UVB rays. histochemical and biochemical study. Histol.Histopathol. 2000;15:10431050.

355. Larrosa M, Lodovici M, Morbidelli L, Dolara P. Hydrocaffeic and p-coumaric acids, natural phenolic compounds, inhibit UV-B damage in WKD human conjunctival cells in vitro and rabbit eye in vivo. Free Radic.Res. 2008;42:903-910.

356. Chen MH, Tsai CF, Hsu YW, Lu FJ. Epigallocatechin gallate eye drops protect against ultraviolet B-induced corneal oxidative damage in mice. Mol.Vis. 2014;20:153-162.

357. Bretscher P, Cohn M. A theory of selfnonself discrimination. Science 1970;169:1042-1049.

358. Lugli E, Ferraresi $R$, Roat $E$, et al. Quercetin inhibits lymphocyte activation and proliferation without inducing apoptosis in peripheral mononuclear cells. Leuk.Res. 2009;33:140-150.
359. Sternberg Z, Chadha K, Lieberman A, et al. Quercetin and interferon-beta modulate immune response(s) in peripheral blood mononuclear cells isolated from multiple sclerosis patients. J.Neuroimmunol. 2008;205:142-147.

360. Tan $W F$, Lin LP, $\mathrm{Li} M \mathrm{MH}$, et al. Quercetin, a dietary-derived flavonoid, possesses antiangiogenic potential. Eur.J.Pharmacol. 2003;459:255-262.

361. Larocca LM, Teofili L, Leone G, et al. Antiproliferative activity of quercetin on normal bone marrow and leukaemic progenitors. Br.J.Haematol. 1991;79:562566.

362. Falchetti R, Fuggetta MP, Lanzilli G, Tricarico M, Ravagnan $G$. Effects of resveratrol on human immune cell function. Life Sci. 2001;70:81-96.

363. Boscolo P, del Signore A, Sabbioni E, et al. Effects of resveratrol on lymphocyte proliferation and cytokine release. Ann.Clin.Lab.Sci. 2003;33:226-231.

364. Larbi A, Kempf J, Pawelec G. Oxidative stress modulation and $\mathrm{T}$ cell activation. Exp.Gerontol. 2007;42:852-858.

365. Cosentino M, Bombelli R, Carcano E, et al. Immunomodulatory properties of achyrocline satureioides (lam.) D.C. infusion: A study on human leukocytes. J.Ethnopharmacol. 2008;116:501-507.

366. Arredondo MF, Blasina F, Echeverry $\mathrm{C}$, et al. Cytoprotection by achyrocline satureioides (lam) D.C. and some of its main flavonoids against oxidative stress. J.Ethnopharmacol. 2004;91:13-20.

367. Corrales RM, Villarreal A, Farley W, Stern ME, Li DQ, Pflugfelder SC. Strainrelated cytokine profiles on the murine ocular surface in response to desiccating stress. Cornea 2007;26:579-584.

368. Lee HS, Chauhan SK, Okanobo A, Nallasamy N, Dana R. Therapeutic efficacy of topical epigallocatechin gallate in murine dry eye. Cornea 2011. 
369. Barabino S, Chen W, Dana MR. Tear film and ocular surface tests in animal models of dry eye: Uses and limitations. Exp.Eye Res. 2004;79:613-621.

370. Pitcher JD,3rd, De Paiva CS, Pelegrino FS, et al. Pharmacological cholinergic blockade stimulates inflammatory cytokine production and lymphocytic infiltration in the mouse lacrimal gland. Invest.Ophthalmol.Vis. Sci. 2011;52:3221-3227.

371. Inaba T, Shimazaki J, Tanaka Y, Tsubota K. The potential of quercetin for dry eye diseases. Invest.Ophthalmol.Vis.Sci. 2014;55:3659.

372. Corrales RM, Stern ME, De Paiva CS, Welch J, Li D, Pflugfelder SC. Desiccating stress stimulates expression of matrix metalloproteinases by the corneal epithelium. Invest.Ophthalmol.Vis.Sci. 2006;47:3293-3302.

373. Wilson SE, Esposito A. Focus on molecules: Interleukin-1: A master regulator of the corneal response to injury. Exp.Eye Res. 2009;89:124-125.

374. Choi W, Li Z, Oh HJ, et al. Expression of CCR5 and its ligands CCL3, -4 , and -5 in the tear film and ocular surface of patients with dry eye disease. Curr.Eye Res. 2012;37:12-17.

375. Stern ME, Schaumburg CS, Dana R, Calonge M, Niederkorn JY, Pflugfelder SC. Autoimmunity at the ocular surface: Pathogenesis and regulation. Mucosal Immunol. 2010;3:425-442.

376. Held KS, Schaumburg CS, Gao J, et al. Regulatory $\mathrm{T}$ cell mediated suppression of dendritic cells in a mouse model of dry eye. Invest.Ophthalmol.Vis.Sci. 2014;55:3653.

377. Stern ME, Held KS, Schaumburg CS, et al. Stress-induced damage to the ocular surface resembling dry eye can occur independently of chronic $T$ cell-mediated disease. Invest.Ophthalmol.Vis.Sci. 2014:55:3059.

378. Bax HJ, Keeble AH, Gould HJ. Cytokinergic IgE action in mast cell activation. Front.Immunol. 2012;3:229.
379. Kimata $M$, Shichijo $M$, Miura $T$, Serizawa I, Inagaki N, Nagai $\mathrm{H}$. Effects of luteolin, quercetin and baicalein on immunoglobulin E-mediated mediator release from human cultured mast cells. Clin.Exp.Allergy 2000;30:501-508.

380. Baolin L, Inami $\mathrm{Y}$, Tanaka $\mathrm{H}$, Inagaki $\mathrm{N}$, linuma $\mathrm{M}$, Nagai $\mathrm{H}$. Resveratrol inhibits the release of mediators from bone marrowderived mouse mast cells in vitro. Planta Med. 2004;70:305-309.

381. Mohd Zaki A, Galatowicz G, Calder VL, Ocular Immunology . Enhanced expression of conjunctival IL-9 co-localized with mast cells in seasonal allergic conjunctivitis and its effect on mast cell cytokine secretion via IL-9R. Invest.Ophthalmol.Vis.Sci. 2014;55:2484.

382. Min YD, Choi $\mathrm{CH}$, Bark $\mathrm{H}$, et al. Quercetin inhibits expression of inflammatory cytokines through attenuation of NF-kappaB and p38 MAPK in HMC-1 human mast cell line. Inflamm.Res. 2007;56:210-215.

383. Park HH, Lee $S$, Son HY, et al. Flavonoids inhibit histamine release and expression of proinflammatory cytokines in mast cells. Arch.Pharm.Res. 2008;31:13031311.

384. Kempuraj D, Madhappan B, Christodoulou S, et al. Flavonols inhibit proinflammatory mediator release, intracellular calcium ion levels and protein kinase $C$ theta phosphorylation in human mast cells. Br.J.Pharmacol. 2005;145:934944.

385. Kang $\mathrm{OH}$, Jang $\mathrm{HJ}$, Chae HS, et al. Anti-inflammatory mechanisms of resveratrol in activated HMC-1 cells: Pivotal roles of NF-kappaB and MAPK. Pharmacol.Res. 2009;59:330-337.

386. Catalli A, Karpov V, Pundir P, Dimitrijevic A, Kulka M. Comparison of the inhibitory effects of resveratrol and tranilast on $\operatorname{IgE}, 48 / 80$ and substance $P$ dependentmast cell activation. Allergy, Asthma \& Clinical Immunology 2010;6:1-1. 
387. Cruz EA, Da-Silva SA, Muzitano MF, Silva PM, Costa SS, Rossi-Bergmann B. Immunomodulatory pretreatment with kalanchoe pinnata extract and its quercitrin flavonoid effectively protects mice against fatal anaphylactic shock. Int.Immunopharmacol. 2008;8:1616-1621.

388. Pflugfelder SC. Antiinflammatory therapy for dry eye. Am.J.Ophthalmol. 2004;137:337-342.

389. Nakamura S, Shibuya M, Nakashima $\mathrm{H}$, et al. Involvement of oxidative stress on corneal epithelial alterations in a blinksuppressed dry eye. Invest.Ophthalmol.Vis.Sci. 2007;48:15521558.

390. Halestrap AP, McStay GP, Clarke SJ. The permeability transition pore complex: Another view. Biochimie 2002;84:153-166.

391. Green DR, Evan GI. A matter of life and death. Cancer.Cell. 2002;1:19-30.

392. Pourzand C, Tyrrell RM. Apoptosis, the role of oxidative stress and the example of solar UV radiation. Photochem.Photobiol. 1999;70:380-390.

393. Gao J, Sana R, Calder V, et al. Mitochondrial permeability transition pore in inflammatory apoptosis of human conjunctival epithelial cells and $T$ cells: Effect of cyclosporin A. Invest.Ophthalmol.Vis. Sci. 2013;54:47174733. 